\title{
CONNECTIVITY OF WIRELESS SENSOR NETWORKS USING DIRECTIONAL ANTENNAE
}

\author{
by \\ Oscar Morales Ponce \\ A Thesis Submitted to \\ the Faculty of Graduate and Post Doctoral Affairs \\ in Partial Fulfillment of the Requirements for the Degree of \\ DOCTOR OF PHILOSOPHY \\ School of Computer Science \\ at \\ CARLETON UNIVERSITY
}

Ottawa, Ontario

May, 2011

(C) Copyright by Oscar Morales Ponce, 2011 
Library and Archives

Canada

Published Heritage

Branch

395 Wellington Street

Ottawa ON K1A ON4

Canada
Bibliothèque et

Archives Canada

Direction du

Patrimoine de l'édition

395, rue Wellington

Ottawa ON K1A ON4

Canada
Your file Votre référence

ISBN: 978-0-494-81585-4

Our file Notre référence

ISBN: 978-0-494-81585-4
NOTICE:

The author has granted a nonexclusive license allowing Library and Archives Canada to reproduce, publish, archive, preserve, conserve, communicate to the public by telecommunication or on the Internet, loan, distribute and sell theses worldwide, for commercial or noncommercial purposes, in microform, paper, electronic and/or any other formats.

The author retains copyright ownership and moral rights in this thesis. Neither the thesis nor substantial extracts from it may be printed or otherwise reproduced without the author's permission.
AVIS:

L'auteur a accordé une licence non exclusive permettant à la Bibliothèque et Archives Canada de reproduire, publier, archiver, sauvegarder, conserver, transmettre au public par télécommunication ou par l'Internet, prêter, distribuer et vendre des thèses partout dans le monde, à des fins commerciales ou autres, sur support microforme, papier, électronique et/ou autres formats.

L'auteur conserve la propriété du droit d'auteur et des droits moraux qui protège cette thèse. $\mathrm{Ni}$ la thèse ni des extraits substantiels de celle-ci ne doivent être imprimés ou autrement reproduits sans son autorisation.
In compliance with the Canadian Privacy Act some supporting forms may have been removed from this thesis.

While these forms may be included in the document page count, their removal does not represent any loss of content from the thesis.
Conformément à la loi canadienne sur la protection de la vie privée, quelques formulaires secondaires ont été enlevés de cette thèse.

Bien que ces formulaires aient inclus dans la pagination, il n'y aura aucun contenu manquant. 
To my lovely mother, Berta Ponce. 


\section{Abstract}

In this thesis, we study fundamental issues on sensor networks employing directional antennae. The main question that motivates this work is how omnidirectional antennae can be replaced with directional antennae in such a way that the connectivity is maintained while the angle and range are minimum. This leads to the natural problem of finding the minimum radius to achieve connectivity for a given angle when each sensor has $k \geq 1$ directional antennae. We prove that for any given angle a strongly connected network can be created with radius bounded by $2 \cdot \sin \left(\frac{\pi}{k+1}\right)$ times the optimal radius when $k \leq 5$. We also prove a lower bound when 2 antennae are being used per sensor. A slightly different problem is to replace omnidirectional antennae of a given network with the minimum number of directional antennae while the minimum path between any two points in the resulting network (hop-stretch factor) increases independently of the size of the network. We give upper bounds on the number of directional antennae and hop-stretch factor for this problem provided we have an underlying two-edge connected geometric planar graph. This leads to the problem of constructing 2-edge connected geometric planar graphs with bounded edge length. Two approaches can be considered: i) Augmenting the connectivity of a given planar graph by adding new edges of bounded length. Using this approach, we give an upper bound on the number of new edges of bounded length to augment the connectivity of a given geometric planar graph into a 2-edge connected planar graph. We prove that determining the minimum number of new edges of bounded length to augment the connectivity is intractable. ii) Obtaining a 2-edge connected planar subgraph of a given Unit Disk Graph (UDG). We prove that deciding whether a UDG has a planar subgraph of degree two is intractable even for a scaling factor of $\frac{\sqrt{5}}{2}$. On the opposite side, we prove that the square of any 2-edge connected UDG always has an underlying 2-edge connected geometric planar subgraph. 


\section{Acknowledgements}

I owe my deepest gratitude to my supervisor Evangelos Kranakis for all his constant effort and patience during my $\mathrm{PhD}$. His advice has been of great help to the culmination of this thesis. It is a pleasure to thank everyone who has collaborated on the research done in this thesis. I would also like to thank my family for their support. I am grateful to Mexican National Council of Science and Technology (CONACYT). Without their grant, this $\mathrm{PhD}$ would not have been possible. Lastly but not least, I would like to express my deepest gratitude to my lovely wife for her patience and support. 


\section{Table of Contents}

Abstract

Acknowledgements iv

$\begin{array}{lc}\text { List of Tables } & \text { ix }\end{array}$

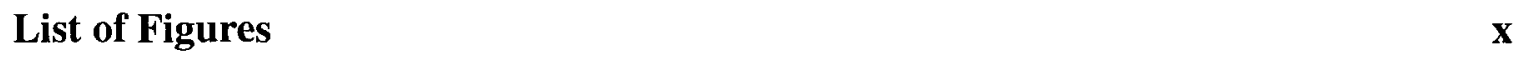

$\begin{array}{lll}\text { Chapter } 1 \text { Introduction } & \mathbf{1}\end{array}$

1.1 Replacing Omnidirectional with Directional Antennae . . . . . . . . 2

1.2 Enhancing Network Performance using Directional Antennae . . . . . . . 4

1.3 Other Applications . . . . . . . . . . . . . . . . 5

1.4 Preliminaries and Notation .................. 5

1.4.1 Euclidean Minimum Spanning Tree . . . . . . . . . . . 6

1.5 Problems Investigated . . . . . . . . . . . . . . . . . 7

1.5.1 Antenna Orientation Problem ............. 7

1.5.2 Graph Orientation Problem . . . . . . . . . . . . . 8

1.5.3 Augmentation Problem with Bounded Length . . . . . . . . 8

1.5.4 Min Degree Planar Subgraph Problem . . . . . . . . . . 8

1.5.5 Optimal Length $k$-Connected Planar Graph Problem . . . . . . . 9

1.6 Summary of Results . . . . . . . . . . . . . . . . . . . 9

1.6.1 Antenna Orientation Problem ............ . 9

1.6.2 Antenna Orientation Problem In 3D . . . . . . . . . . . 10

1.6.3 Graph Orientation Problem . . . . . . . . . . . . . . 11

1.6.4 Augmentation Problem with Bounded Length . . . . . . . . . . . 11

1.6.5 Min Degree Planar Subgraph Problem . . . . . . . . . . 12

1.6.6 Optimal Length $k$-Connected Planar Graph Problem . . . . . . . 13

1.7 Publications . . . . . . . . . . . . . . . . . . . . 13 
$\begin{array}{lll}\text { Chapter } 2 & \text { Related Work } & 16\end{array}$

2.1 Antenna Orientation Problem . . . . . . . . . . . . . . 16

2.2 Graph Orientation Problem . . . . . . . . . . . . . 17

2.3 Augmentation Problem with Bounded Length . . . . . . . . . . . . . . 18

2.4 Min Degree Planar Subgraph Problem . . . . . . . . . . . . . . . 19

2.5 Optimal Length $k$-Connected Planar Graph Problem . . . . . . . . . . . . 19

$\begin{array}{lll}\text { Chapter } 3 & \text { Antenna Orientations }\end{array}$

3.1 Introduction . . . . . . . . . . . . . . . 20

3.1.1 Preliminaries and Notation . . . . . . . . . . . 20

3.1 .2 Our Results . . . . . . . . . . . . . . 21

3.2 Upper Bound Results on Strongly Connected Spanning Digraphs . . . . . 21

3.2 .1 Maximum Out-Degree $4 \ldots . \ldots 23$

3.2.2 Maximum Out-Degree $3 \ldots \ldots 25$

3.2.3 Maximum Out-Degree $2 \ldots \ldots 28$

3.2 .4 Algorithm ......................... 39

3.3 NP hardness . . . . . . . . . . . . . . . . . 40

3.4 Sum of Angles . . . . . . . . . . . . . . . . . 46

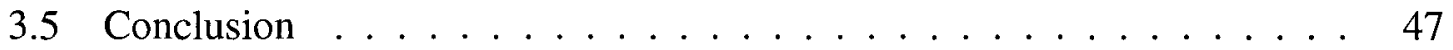

$\begin{array}{lll}\text { Chapter } 4 & \text { Antenna Orientations in 3D }\end{array}$

4.1 Introduction . . . . . . . . . . . . . . . . 48

4.1.1 Preliminaries and Notation . . . . . . . . . . . 48

4.1 .2 Our Results ... . . . . . . . . . . . . . 49

4.2 Lower Bound on Solid Angle and Optimal Range . . . . . . . . . . . 50

4.3 Approximation Algorithm and Upper Bound . . . . . . . . . . . . 52

4.4 The Complexity of the $3 \mathrm{D}$ Case . . . . . . . . . . . . . 54

4.5 Simulation Results . . . . . . . . . . . . . . . . 55

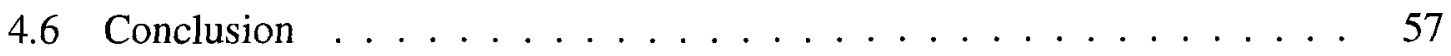


Chapter $5 \quad$ Orientation of 2-Edge Connected Planar Graph 58

5.1 Introduction . . . . . . . . . . . . . . . 58

5.1 Preliminaries and Notation . . . . . . . . . . . . 58

5.1 .2 Our Results . . . . . . . . . . . . . . . 59

5.2 Orientations with more than $|E|$ Arcs . . . . . . . . . . . . . 59

5.3 Orientations with $|E|$ Arcs $\ldots \ldots \ldots \ldots$. . . . . . . . . . . 63

5.4 Orientations with less than $|E|$ arcs . . . . . . . . . . . . . 66

5.5 Conclusion ........................... 69

Chapter 6 Length 3-Times Optimal, 2-Edge Augmentation of Planar Graphs 70

6.1 Introduction . . . . . . . . . . . . . 70

6.1 .1 Our Results . . . . . . . . . . . . . . . 70

6.2 Augmentation with Bounded Length Edges . . . . . . . . . . . . 71

6.2 .1 Upper bounds . . . . . . . . . . . . . 71

6.2.2 NP Completeness . . . . . . . . . . . . . . 79

6.3 Impossibility of Local Algorithm for Augmentation . . . . . . . . . . . 80

6.4 Constant Time Augmentation in Location Aware Settings . . . . . . . . . 84

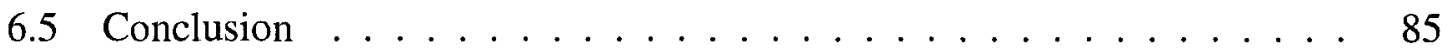

$\begin{array}{lll}\text { Chapter } 7 & \text { Min Degree Planar Subgraphs } & 86\end{array}$

7.1 Introduction . . . . . . . . . . . . . . . 86

7.1 .1 Our Results . . . . . . . . . . . . . . . 87

7.2 Orientation Problem on Planar Circuit Networks . . . . . . . . . . . 87

7.3 Orientation Problem . . . . . . . . . . . . . . . 88

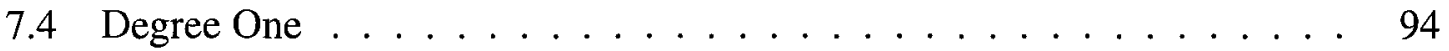

7.5 Degree Two . . . . . . . . . . . . . . . 96

7.6 Degree Three . . . . . . . . . . . . . . . . . . 99

7.7 Conclusion . . . . . . . . . . . . . . . . . . . 99

Chapter 8 Length 2-Times Optimal, 2-Edge Connected Planar Graph 101

8.1 Introduction . . . . . . . . . . . . . . 101 
8.1 .1 Our Results . . . . . . . . . . . . . . . . . . . . . 102

8.2 Planar Graph of Points in a Narrow Strip with Bounded Length Edges . . 102

8.3 General Concepts . . . . . . . . . . . . . . . . . . . . . . 103

8.4 Planar Graph of UDGs with Minimum Degree $2 \ldots \ldots$

8.4 .1 Lower Bound . . . . . . . . . . . . . . . . . . . 106

8.4 .2 Upper Bound . . . . . . . . . . . . . . . . . . . . . 107

8.5 2-Edge Connected Planar Graph of UDGs with Minimum Degree $2 \ldots \ldots$

8.5 .1 Lower Bound . . . . . . . . . . . . . . . . . . . 116

$8.5 .2 \quad$ Upper Bound . . . . . . . . . . . . . . . . . . . . 117

8.6 2-Edge Connected Planar Graph of a 2-Edge Connected UDG . . . . . . 118

8.6 .1 Lower Bound . . . . . . . . . . . . . . . . . . . . . . . . . . . . 119

8.6.2 Upper Bound . . . . . . . . . . . . . . . . . . . . . . . . . . . 119

8.7 Two-Edge Connected Planar Graphs of UDGs with high connectivity . . . 125

8.8 Conclusion . . . . . . . . . . . . . . . . . 127

$\begin{array}{lll}\text { Chapter } 9 & \text { Conclusion and Further Research } & 128\end{array}$

$\begin{array}{ll}\text { Nomenclature } & 131\end{array}$

$\begin{array}{ll}\text { Index } & 132\end{array}$

$\begin{array}{ll}\text { Bibliography } & 133\end{array}$ 


\section{List of Tables}

1.1 Results of the antenna orientation problem in 2D presented in Chapter $3 . \ldots \ldots \ldots \ldots \ldots \ldots$

1.2 Results of the antenna orientation problem in 3D with one antenna per sensor presented in Chapter 4. . . . . . . . . . . . . 10

1.3 Results of the graph orientation problem presented in Chapter 5. . . 11

1.4 Results of the augmentation problem with bounded length presented in Chapter 6. . . . . . . . . . . . . . . . . . . . . . 12

1.5 Computational complexity of finding a planar spanning subgraph that has a given minimum degree in different families of geometric graphs. . . . . . . . . . . . . . . . 13

1.6 Computational complexity of the augmentation problem. . . . . 13

$1.7 \quad$ Results of the optimal length $k$-Connected planar graphs problem presented in Chapter 8. . . . . . . . . . . . . . . . . 14

$2.1 \quad$ Results given in $[10]$ and $[7] \ldots \ldots \ldots \ldots$

3.1 Trade-off between the number, max range and sum of angles when $k$ antennae are being used by each sensor. . . . . . . . . . . . 47

9.1 Conjectured values for the antenna orientation problem. $\ldots \ldots$. . 128 


\section{List of Figures}

Antenna Model. ................... 2

1.2 Network formed with four sensors. . . . . . . . . . . . 3

1.3 Four sensors using directional antennae. Using three directional antennae per sensor in order to form an underlying complete network on four nodes. . . . . . . . . . . . . . . 3

1.4 Line graph network with undirected edges $\{1,2\},\{2,3\},\{3,4\}$ resulting when four sensors $1,2,3,4$ use omnidirectional antennae. .

1.5 Directed network resulting from Figure 1.4 when the four sensors replace omnidirectional with directional antennae. Sensor number 3 is using two directional antennae while the rest only one. . . . .

$3.1 \quad$ An A-Tree with 7 nodes. . . . . . . . . . . 22

3.2 $T$ is a tree with five leaves and diameter $l=2$. (The angular sign with a dot depicts an angle of size at most $2 \pi / 5$.) . . . . . . .

3.3 Depicting the inductive step when $u$ has four neighbors in $T \backslash T^{\prime}$. (The dashed edge $\left\{u_{0}, u\right\}$ indicates that it does not exist in $G$ but exists in $G^{\prime}$, the angular sign with a dot depicts an angle of size at most $2 \pi / 5$, and the dotted curve is used to separate $T^{\prime}$ from $T$.) . .

3.4 $T$ is a tree of diameter $l=2$. (The angular sign with a dot depicts an angle of size at most $\pi / 2$ and the angular sign depicts an angle of size greater than $\pi / 2.) \ldots \ldots \ldots$

3.5 Depicting the inductive step when $u$ has three neighbors in $T \backslash T^{\prime}$. (The dashed edge $\left\{u, u_{0}\right\}$ indicates that it does not exist in $G$ but exists in $G^{\prime}$, the angular sign with a dot depicts an angle of size at most $\pi / 2$ and the dotted curve is used to separate $T^{\prime}$ from $T$.) . . . 
3.6 Depicting the inductive step when $u$ has four neighbors in $T \backslash T^{\prime}$. (The dashed edge $\left\{u_{0}, u\right\}$ indicates that it does not exist in $G$ but exits in $G^{\prime}$, the angular sign depicts an angle of size greater than $\pi / 2$ and the dotted curve is used to separate the tree $T^{\prime}$ from $T$.) . . 28

3.7 Depicting the case when $\pi / 3 \leq \alpha \leq \pi / 2 \ldots \ldots \ldots 29$

3.8 Depicting the case when $\pi / 2<\alpha \leq \pi / 6+\arccos (1 / 2 \sqrt{3}) . \ldots 30$

3.9 Depicting the case when $\pi / 6+\arccos (1 / 2 \sqrt{3})<\alpha \leq 2 \pi / 3$. . . 30

3.10 Cones $\mathcal{C}, \mathcal{D}, \mathcal{E}$ with apex at $v \ldots \ldots \ldots 32$

3.11 Depicting when $d(v)=4$, two consecutive children of $v$ are far and all the children are at distance at least $\sqrt{3}-1$ from $v$. . . . . .

3.12 $T$ is a tree with diameter $l=2$. (The heavy arrows represent the newly added edges, the angular sign with a dot depicts an angle of size at most $2 \pi / 3$ and dashed edge indicates that it exists in $T$ but not in $G$.) . . . . . . . . . . . . . . . .

3.13 Depicting the inductive step when $u$ has two neighbors in $T \backslash T^{\prime}$. (The dashed edge $\left\{u_{0}, u\right\}$ indicates that it does not exist in $G$ but exists in $G^{\prime}$ and the dotted curve is used to separate $T^{\prime}$ from $T$.) .

3.14 Depicting the inductive step when $u$ has two neighbors in $T \backslash T^{\prime}$, $u_{0}$ and $u_{1}$ are far and $\left\{u_{0}, u\right\}$ is not in $G^{\prime}$. (The dashed edge $\{v, u\}$ indicates that it does not exist in $G$ but exists in $G^{\prime}$, the dash dotted edge $\left\{u_{0}, u\right\}$ indicates that it exists in $T^{\prime}$ but not in $G^{\prime}$ and the dotted curve is used to separate $T^{\prime}$ from $T$.) . . . . . . . . . 36

3.15 Depicting the inductive step when $u$ has three neighbors in $T \backslash T^{\prime}$, $u_{1}$ and $u_{2}$ are far and $\left\{u_{0}, u\right\}$ is in $G^{\prime}$. (The dashed edge $\left\{u_{0}, u\right\}$ indicates that it does not exist in $G$ but exists in $G^{\prime}$ and the dotted curve is used to separate $T^{\prime}$ from $T$.) . . . . . . . . . . 
3.16 Depicting the inductive step when $u$ has three neighbors in $T \backslash T^{\prime}$, $u_{1}$ and $u_{2}$ are far and $\left\{u_{0}, u\right\}$ is not in $G^{\prime}$. (The dashed edge $\{v, u\}$ indicates that it does not exist in $G$ but exists in $G^{\prime}$, the dash dotted edge $\left\{u_{0}, u\right\}$ indicates that it exists in $T^{\prime}$ but not in $G^{\prime}$ and the dotted curve is used to separate $T^{\prime}$ from $T$.) . . . . . . . . . .

3.17 Depicting the inductive step when $u$ has four neighbors in $T \backslash T^{\prime}$, $\left\{u_{0}, u\right\}$ is in $G^{\prime}$. (The dashed edge $\left\{u_{0}, u\right\}$ indicates that it does not exist in $G$ but exists in $G^{\prime}$, the dotted curve is used to separate $T^{\prime}$ from $T$ and the dash dotted edge $\left\{u, u_{3}\right\}$ indicates that it exists in $T$ but not in G.) . . . . . . . . . . . . . . . . . . 38

3.18 Depicting the inductive step when $u$ has four neighbors in $T \backslash T^{\prime}$ and $\left\{u_{0}, u\right\}$ is not in $G^{\prime}$. (The dashed edge $\{v, u\}$ indicates that it does not exist in $G$ but exists in $T^{\prime}$, the dotted curve is used to separate $T^{\prime}$ from $T$, the dash dotted edge $\left\{u_{0}, u\right\}$ indicates that it exists in $T^{\prime}$ but not in $G^{\prime}$ and the dash dotted edge $\left\{u, u_{3}\right\}$ indicates that it exist in $T$ but not in G.) . . . . . . . . . . . . . . . 39

3.19 Meta-vertex and meta-edge for the NP hardness proof. . . . . . . 42

3.20 Connecting meta-edges with meta-vertices. The dashed ovals show the places where embedding is constrained. . . . . . . . . . 43

$3.21 \quad$ Antenna assignments in meta-edges. . . . . . . . . . . . 44

3.22 Antenna assignments at the meta-vertex and incident meta-vertices. 44

4.1 3D directional antenna of solid angle $\Omega=2 \pi(1-\cos \theta) \ldots \ldots 49$

$4.2 \quad$ Tammes' problem. . . . . . . . . . . . . . . 50

$4.3 \quad$ A circumcircle and the Tammes radius $R_{12} \ldots \ldots \ldots 52$

4.4 Orientation of antennae at $u$ and $v$ such that $(u, v)$ is in the matching

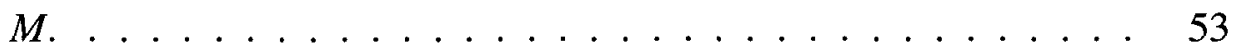

4.5 Directed Transmission Graph/Unit Ball Graph varying n. (Hop stretch factor of the average shortest paths.) $\ldots \ldots \ldots . . .56$ 
4.6 Directed Transmission Graph/Unit Ball Graph varying $\Omega$. (Hop stretch factor of the average shortest paths.) $\ldots \ldots \ldots \ldots$

5.1 The figure shows how to find a directed path from $v$ to $u$ when $(u, v)$ is in $D(G ; \Lambda)$. If $\{a, b\}$ is any edge incident to $L_{u, v}$ then by inductive hypothesis there is a path from $a$ to $b$ of length at most $(\Phi-1)^{l}$.

6.1 A graph of $n$ vertices that requires $\lfloor n / 2\rfloor$ new edges to augment it into a two-edge connected planar graph. . . . . . . . . . 71

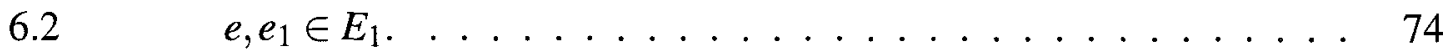

6.3 The removal of $e_{1}$ and $e_{2}$ leaves a chain of four edges in $E_{1} \ldots 75$

6.4 A planar graph that can be augmented to a 2-edge connected planar graph only by adding an edge of length $3-\varepsilon \ldots \ldots \ldots 75$

6.5 If we allow crossings, it can be augmented to a 2-edge connected graph by adding two edges of length $2-\varepsilon \ldots \ldots \ldots 76$

6.6 $n$ nodes arranged in a convex line graph. For each $i$, node $i$ has identity $i d_{i} \ldots \ldots \ldots \ldots \ldots$. . . . . . . . . . 80

6.7 Pairing nodes of the line graph so as to form a 2-edge connected planar augmentation. . . . . . . . . . . . . . 81

6.8 Planar representation of the line graph augmented with new edges. $\quad 83$

$6.9 v$ is an internal vertex of a convex line graph with respect to $u$ and $w$ and the end of two convex line graphs. (Solid lines represent original edges, dashed lines represent new added edges and dotted lines show the convex line graphs that the original graph forms.) .

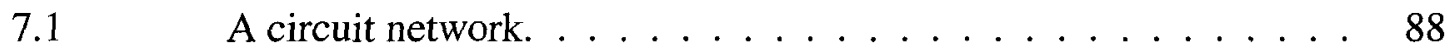

7.2 (a) Valid and (b) invalid orientations of a circuit network. . . . . 89

7.3 Connection between clauses and variables $\left(e_{x}(i)\right.$ is a positive edge and $e_{x}(i+1)$ is a negative edge). . . . . . . . . 92

7.4 Gadgets for qUDG $(1-\varepsilon)$ and minimum degree 1: (a) wire, (b) switch, (c) user. (d) Embedding of a switch, three ports, and three wires. . 95 
7.5 Gadgets for orthogonal graphs: (a) wire, (b) switch, (c) user. . . 96

7.6 Gadgets for UDG, minimum degree 2: (a) wire, (b) switch, (c) user,

(d) terminal. . . . . . . . . . . . . . . . . . . . . . . 98

7.7 Gadgets for UDG and minimum degree 3: (a) wire, (b) switch,

(c) user. (d) Embedding of a switch and three wires. . . . . . . . 100

$8.1 \quad \operatorname{Tie}(u ; v, x, y)$ with $\operatorname{tip} u \ldots \ldots \ldots \ldots 104$

8.2 If $u, v, x, y$ form a $\operatorname{Tie}(u ; v, x, y)$ then $\angle(y v x) \geq 2 \pi / 3 \ldots \ldots \ldots$

8.3 If $u, v, x, y$ form a $\operatorname{Tie}(u ; v, x, y)$ then $u^{\prime}$ cannot form a Tie with either $v$ or $x$ or $y$ that overlaps $\operatorname{Tie}(u ; v, x, y) . \ldots \ldots \ldots$

$8.4 \quad$ In a Tie $(u ; v, x, y) d(u, x) \leq \sqrt{2}$ and $d(u, y) \leq \sqrt{2} \ldots \ldots \ldots 6$

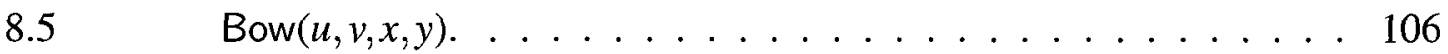

8.6 UDG of minimum degree two that requires scaling factor of $2 . \quad \ldots 107$

$8.7 \quad$ A SNN crossing an edge of the MST . . . . . . . . . 108

$8.8 \quad$ Two SNN edges crossing. . . . . . . . . . . . . . . . . 109

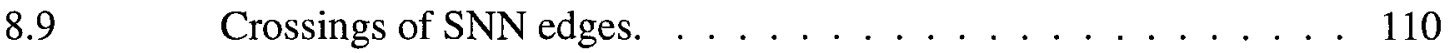

$8.10 \quad$ If $\angle(w u v) \leq \varphi$ then $d\left(w, u^{\prime}\right)^{2}<3-2 \sqrt{2} \cos \left(\varphi-\frac{\pi}{4}\right) \ldots \ldots 111$

$8.11\{u, v\}$ is in at most two Ties. (Solid lines are edges of $T$ and dashed arrow lines are SNN edges.) . . . . . . . . . . . . . . 112

8.12 $\{u, v\}$ is in one Tie. (Dotted lines are removed edges and dashed lines are possible new edges.) . . . . . . . . . . . . . . . . . 113

8.13 $\{u, v\}$ crosses at least one edge of $G$. (Dotted lines are removed edges and dashed lines are possible new edges.) . . . . . . . . . 113

8.14 UDG Component with minimum degree 2 that requires scaling factor of $\sqrt{5} \ldots \ldots \ldots \ldots \ldots \ldots \ldots \ldots$

$8.15 \quad \angle(w u v)<\pi$ and $\left\{u, v^{\prime}\right\} \in E^{\prime} \ldots \ldots \ldots \ldots \ldots \ldots 117$

8.16 Two-edge connected UDG with $1+3 k$ vertices that requires scaling factor of 2. . . . . . . . . . . . . . . . . . . . . . . . . . . . . . 119

$8.17 \quad$ Removing of black arduous vertices. . . . . . . . . . 121 
8.18 $\{u, v\}$ is in at most two Ties. (Solid lines are edges of $T$ and dashed arrow lines are SNN edges.) . . . . . . . . . . . . . 122

$8.19 \quad\{w, v\} \in E^{\prime}$ and $w$ is black. . . . . . . . . . . . 124

$8.20 \quad k$-vertex connected UDG that does not have 2-edge connected planar subgraph. . . . . . . . . . . . . . . . . 127 


\section{Chapter 1}

\section{Introduction}

Wireless Sensor Networks (WSN) consist of a set of small devices with limited computational power called sensors capable of sensing relevant information. Sensors are equipped with wireless antennae which make them able to communicate with other nodes. The objective of WSN is to sense the environment of an area of interest and send the collected information to a sink or share it with its neighbors to perform some computation. What is more, sensors must respond to external queries. However, infrastructure is not always available or its deployment can be prohibited. Therefore, WSN must be self-configurable, adapt dynamically to failures and react to changes in the network. These characteristics make WSNs suitable for a wide variety of applications. For a comprehensive survey of WSN applications we refer to [45] and [75].

Connectivity in wireless sensor networks is established using either omnidirectional or directional antennae. The former transmit signals in all directions while the latter within a limited predefined angle. Directional antennae can be more efficient and transmit further in a given direction for the same amount of energy than omnidirectional ones. This is due to the fact that, to a first approximation, the energy transmission cost of an antenna is proportional to its coverage area. To be more specific, the coverage area of an omnidirectional antenna with range $r$ is generally modelled by a circle of radius $r$ as depicted in Figure 1.1a and consumes energy proportional to $\pi \cdot r^{2}$. By contrast, a directional antennae with angular spread $\varphi$ and range $R$ is modelled as a circular sector of angle $\varphi$ and radius $R$ as depicted in Figure $1.1 \mathrm{~b}$ and consumes energy proportional to $\varphi \cdot R^{2} / 2$. Thus for a given energy cost $E$, an omnidirectional antenna can reach distance $\sqrt{E / \pi}$, while a directional antenna with angular spread $\varphi$ can reach distance $\sqrt{2 E / \varphi}$.

We think of the directional antennae as being on a "swivel" that can be oriented towards a small target area whereas the omnidirectional antennae spread their signal in all directions. Signals arriving at a sensor within the target area of multiple antennae will 


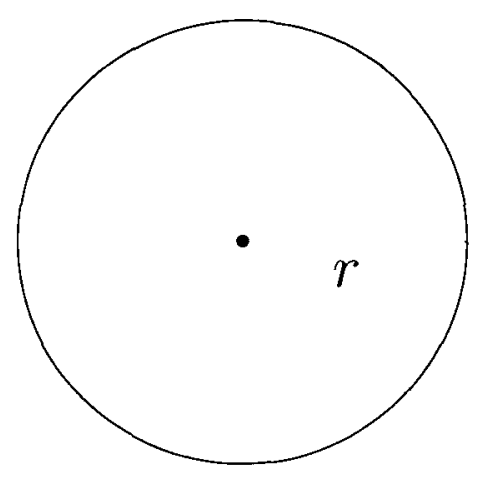

(a) Omnidirectional antenna with radius $r$.

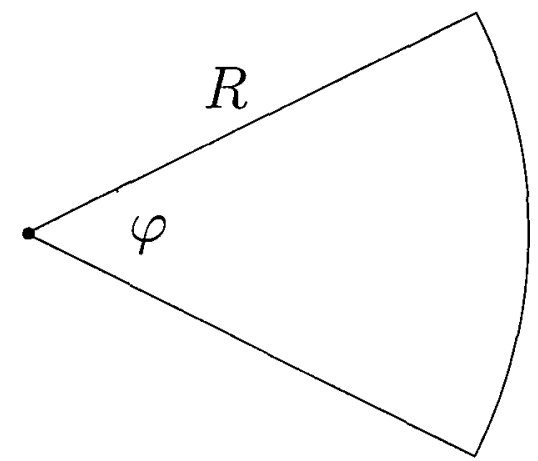

(b) Directional antenna with angle $\varphi$ and radius $R$

Figure 1.1: Antenna Model.

interact (interfere) and therefore the reception is degraded. Thus for reasons of both energy efficiency and potentially reduced interference (as well as others, e.g., security), it is tempting to replace omnidirectional with directional antennae.

\subsection{Replacing Omnidirectional with Directional Antennae}

Given a set of sensors positioned in the plane with omnidirectional and/or directional antennae, a directed network is formed as follows: a directed edge is placed from sensor $u$ to sensor $v$ if $v$ lies within the coverage area of $u$ (as modelled by circles or circular sectors). Note that if the radius of all omnidirectional antennae are the same then $u$ is in the range of $v$ if and only if $v$ is in the range of $u$, i.e., the edge is bidirectional and is usually modelled be an undirected edge.

The main issue of concern when replacing omnidirectional with directional antennae is that this may alter important characteristics such as the degree, diameter, average path length, etc., of the resulting network. For example, the network depicted in Figure 1.2a is strongly connected with diameter four and more than one node can potentially transmit at the same time without interference while in the omnidirectional case (Figure 1.2b) the diameter is one but only one antennae can transmit at a time without interference.

In addition, and depending on the breadth and range of the directional antennae the original topology depicted in Figure $1.2 \mathrm{~b}$ can be obtained only by using more than one directional antenna per sensor (see Figure 1.3). 


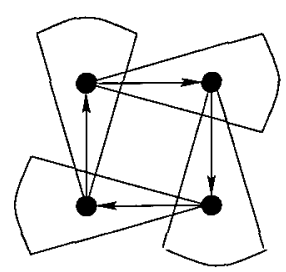

(a) Strongly connected directed network.

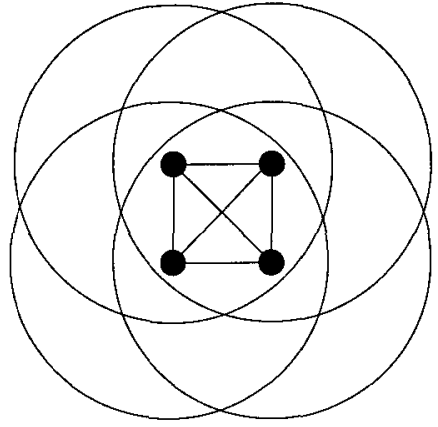

(b) Underlying complete network.

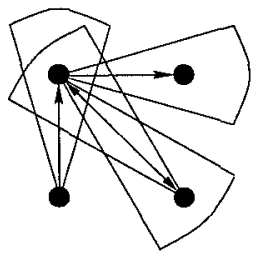

(c) Not strongly connected network.

Figure 1.2: Network formed with four sensors.

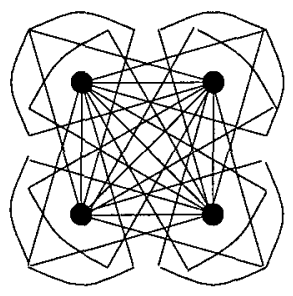

Figure 1.3: Four sensors using directional antennae. Using three directional antennae per sensor in order to form an underlying complete network on four nodes.

Replacing omnidirectional with directional antennae enables the sensors to reach farther using the same energy consumption. As an example consider the graphs depicted in Figures 1.4 and 1.5. The line graph network in Figure 1.4 with undirected edges $\{1,2\},\{2,3\},\{3,4\}$ is replaced by a network of directional antennae depicted in Figure 1.5 and having $(1,2),(1,3),(2,3),(2,4),(3,4),(4,3),(4,2),(3,2),(3,1)$ as directed edges. By setting the angular spread of the directional antennae to be small a significant savings in energy is possible.

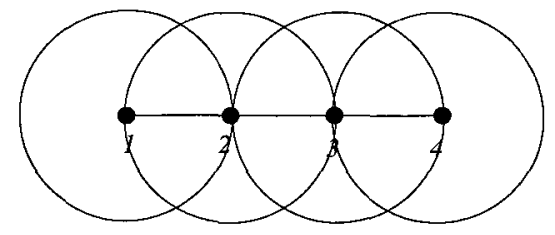

Figure 1.4: Line graph network with undirected edges $\{1,2\},\{2,3\},\{3,4\}$ resulting when four sensors $1,2,3,4$ use omnidirectional antennae. 


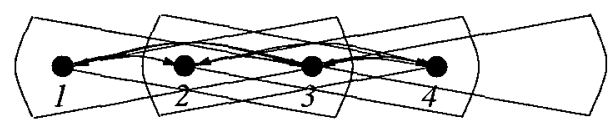

Figure 1.5: Directed network resulting from Figure 1.4 when the four sensors replace omnidirectional with directional antennae. Sensor number 3 is using two directional antennae while the rest only one.

\subsection{Enhancing Network Performance using Directional Antennae}

Directional antennae are known to enhance ad hoc network capacity and performance and when replacing omnidirectional with directional antennae one can reduce the total energy consumption of the network. A theoretical model to this effect is presented in [27] showing that when $n$ omnidirectional antennae are optimally placed and assigned optimally chosen traffic patterns the transport capacity is $\Theta(\sqrt{W / n})$, where each antenna can transmit $W$ bits per second over the common channel(s). When both transmission and reception is directional, [74] proves an $\sqrt{2 \pi / \alpha \beta}$ capacity gain as well as corresponding throughput improvement factors, where $\alpha$ is the transmission angle and $\beta$ is a parameter indicating that $\beta / 2 \pi$ is the average proportion of the number of receivers inside the transmission zone that will get interfered with.

Additional experimental studies confirm the importance of using directional antennae in ad hoc networking for enhancing channel capacity and improving multiaccess control. For example, research in [58] considers several enhancements, including "aggressive" and "conservative" channel access models for directional antennae, link power control and neighbor discovery and analyzes them via simulation. [64] and [65] consider how independent communications between directional antennae can occur in parallel and calculate interference-based capacity bounds for a generic antenna model as well as a real-world antenna model and analyze how these bounds are affected by important antenna parameters like gain and angle. The authors of [4] propose a distributed Receiver-Oriented Multiple Access (ROMA) channel access scheduling protocol for ad hoc networks with directional antennae, each of which can form multiple beams and commence several simultaneous 
communication sessions. Finally, [40] considers energy consumption thresholds in conjunction to $k$-connectivity in networks of sensors with omnidirectional and directional antennae, while [39] studies how directional antennae affect overall coverage and connectivity.

A related problem that has been addressed in the literature is one that studies connectivity requirements on undirected graphs that guarantees highest edge connectivity of its orientation, see for example [23] and [52].

\subsection{Other Applications}

It is interesting to note that beyond reducing the energy consumption, directional antennae can enhance security. Unlike omnidirectional antennae that spread their signal in all directions over an angle $2 \pi$, directional antennae can attain better security because they direct their beam towards the target thus avoiding potential risks along the transmission path. In particular, in a hostile environment a directional antenna can decrease the radiation region within which nodes could receive the electromagnetic signals with high quality. For example, this has led [28] to the design of several authentication protocols based on directional antennae. In [50] the authors employ the average probability of detection to estimate the overall security benefit level of directional transmission over the omnidirectional one. In [29] the possibility of key agreement using variable directional antennae is examined. In [53] the use of directional antennae and beam steering techniques in order to improve performance of links is investigated in the context of communication between a moving vehicle and roadside access points.

\subsection{Preliminaries and Notation}

Definition 1 (Geometric Graph). A geometric graph (or straight line graph) $G=(V, E)$ is a graph where each $v \in V$ is a point in the Euclidean space and each $e \in E$ is a straight line segment joining two distinct points.

Definition $2(U D G(P, r))$. The Unit Disk Graph (or UDG) on a set $P$ of points with parameter $r>0$ is the geometric graph $\operatorname{UDG}(P, r)$ obtained by connecting points with 
straight line segments at Euclidean distance at most $r$. If $r=1$ we simply denote it as $\operatorname{UDG}(P)$.

The following definition

Definition $3(q U D G(P, s)$ [5]). A quasi Unit Disk Graphs (or qUDG) with parameter $s \leq 1$ is the geometric graph qUDG $(P, s)$ defined as follows: two points are incident if the distance is at most $s$. If the distance is greater than s and at most one then they might be incident. Otherwise they are not incident.

Observe that if the parameter $s=1$, then $\operatorname{qUDG}(P, s)=\operatorname{UDG}(P)$. We represent an undirected edge as $\{u, v\}$ and a directed edge as $(u, v)$. The degree of node $v$ in the graph $G$ is denoted by $d_{G}(v)$ (or $d(v)$ if there is no confusion.) A geometric graph is planar if its edges do not cross each other except at their endpoints. $v \in V$ is a cut-vertex of $G$ if the removal of $v$ disconnects $G$. Similarly an edge $\{u, v\} \in E$ is a cut-edge or bridge if the removal of $\{u, v\}$ disconnects $G$. A graph $G$ is called $k$-edge (respectively, $k$-vertex) connected if it remains connected despite the deletion of any $k-1$ edges (respectively, vertices). $k$ connectivity is an important property because it implies fault tolerance under either edge or vertex deletions.

Let $G$ be a graph. An orientation $\vec{G}$ of $G$ is a digraph obtained from $G$ by orienting the edges of $G$. The hop-stretch factor or simply stretch factor of an orientation $\vec{G}$ of $G$ is the minimum value $t$ such that for every path from $u$ to $v$ in $G$ there exists a directed path from $u$ to $v$ in $\vec{G}$ of hop length at most $t$ times the length of the original path.

\subsubsection{Euclidean Minimum Spanning Tree}

Given a weighted graph $G=(V, E)$, the MST of $G$ is a tree that minimizes the sum of weights. The classical algorithms for solving the MST of a graph $G$ were given by Kruskal [44] and Prim [57] which can be implemented in $O(|E| \log (|V|))$ and $O(|E|+$ $|V| \log (|V|))$, respectively. Although an MST cannot be locally constructed since it requires a cycle detection before adding an edge, it can be constructed in a distributed man-

ner in time $O\left(\sqrt{|V|} \log ^{*}(|V|)+d\right)$ where $d$ is the graph diameter [46] and $\log ^{*}(n)$ is the number of steps that the logarithm function is iteratively applied until the result is at most one. 
In the Euclidean version of the MST, the weights of the edges are determined by their Euclidean distances. Given a set $V$ of points in the plane, Shamos [63] observed that the Delaunay Triangulation (DT) of $V$ contains all the edges of the MST of $V$. Since the DT of $V$ can be constructed in $O(|V| \log (|V|))$ and it has $O(|V|)$ edges then an MST on a set of points can be found in $O(|V| \log (|V|))$. In 3-Dimensions, it can be found in $O\left((|V| \log (|V|))^{4 / 3}\right)$ expected time [2].

An interesting property of Euclidean MSTs in $k$-dimensions is that the maximum degree is always bounded by the Kissing Number $[20,56,60]$ in $k$-dimensions. The kissing number is defined as the maximum number of disjoint unit spheres that touch simultaneously a unit sphere. In particular, it is known that the kissing number for two and three dimensions is 6 and 12 respectively. A direct consequence is that the planar angle that two points form with a common parent is at least $\pi / 3$. Therefore, with a simple argument we can construct an MST with maximum degree five in 2-dimensions (2D) [51].

\subsection{Problems Investigated}

The above considerations lead to numerous questions concerning trade-offs between various factors such as connectivity, diameter, interference, etc., when using directional versus omnidirectional antennae in constructing sensor networks. In this thesis, we provide answers and trade-offs to the following questions.

\subsubsection{Antenna Orientation Problem}

The first natural problem arising is how to maintain network connectivity when antenna angles are being reduced while at the same time the transmission range of the sensors is being kept as low as possible. This raises the following optimization problem:

Problem 1. Consider a set $S$ of $n$ points in the plane that can be identified with sensors having a range $r>0$. For a given angle $0 \leq \varphi \leq 2 \pi$ and integer $k$, each sensor is allowed to use at most $k$ directional antennae with sum of angles at most $\varphi$. Determine the minimum range $r$ required so that by appropriately rotating the antennae, a directed, strongly connected network on $S$ is formed. 
An interesting variant of this problem is to consider the points in a 3D space since in a realistic setting, the $2 \mathrm{D}$ model may result in a network that is not strongly connected due to the fact that the sensors may be at distinct altitudes.

\subsubsection{Graph Orientation Problem}

A slight different problem is to optimize the number of directional antennae of a given network in such a way that the connectivity is maintained and the number of hops between two vertices is a multiplicative factor of the original network that does not depend on the size of the original network.

Problem 2. Given a geometric graph $G=(V, E)$, determine the minimum number of oriented arcs of $E$ so that the resulting digraph is strongly connected and the stretch factor does not depend on $|V|$.

Observe that in our problem definition, an edge might be oriented in either one direction or both directions or not even oriented.

We study this problem in 2-edge connected geometric planar graphs which raises the problem of constructing such graphs from a set of points. In what follows, we address this problem from two perspectives.

\subsubsection{Augmentation Problem with Bounded Length}

Given a network, it is of interest to augment its connectivity with the minimum number of straight line edges of bounded length while the properties of the network are preserved.

Problem 3. Given a connected geometric planar graph $G=(V, E)$, find the minimum set $E^{\prime}$ of straight line edges of bounded length such that $G=(V, E \cup$ $\left.E^{\prime}\right)$ is $k$-edge ( $k$-vertex) connected and planar.

\subsubsection{Min Degree Planar Subgraph Problem}

A natural prerequisite in many applications is to decide whether a geometric graph has an underlying planar subgraph with minimum degree $k$ not necessarily connected. This raises the following decision problem. 
Problem 4. Given a geometric graph $G$ and an integer $k$, decide whether $G$ has an underlying planar subgraph with the original embedding such that the minimum degree is at least $k$.

\subsubsection{Optimal Length $k$-Connected Planar Graph Problem}

Given a set $S$ of points in the plane, what is the smallest radius required to construct a $k$-edge ( $k$-vertex) connected planar spanning subgraph?

Problem 5. Given a set $S$ of points in the plane and $k \geq 1$, determine the minimum radius $r$ such that the UDG $(S, r)$ has an underlying $k$-edge ( $k$-vertex) connected planar subgraph.

\subsection{Summary of Results}

In this section we give a summary of the results presented in this thesis.

\subsubsection{Antenna Orientation Problem}

Chapter 3 is devoted to the study of the antenna orientation problem in 2D. The main results presented in Chapter 3 appear in the Proceedings of the 4th Annual International Conference on Combinatorial Optimization and Applications (COCOA'10) [17], also submitted to a special issue of the journal "Discrete Mathematics, Algorithms and Applications" (DMAA), and a book chapter [37].

We prove that given a set $S$ of $n$ sensors in the plane, there is a strongly connected spanning graph on the set of points for each given integer $1 \leq k \leq 5$ so that each sensor uses at most $k$ directional antennae with range $2 \cdot \sin \left(\frac{\pi}{k+1}\right)$ times the optimal range. Moreover, given a minimum spanning tree on the set of points the spanning graph can be constructed in additional $O(n)$ time. In addition, we prove that the problem is NP-Hard when $k=2$, even if the sum of angles is at most $9 \pi / 20$ and the range at most 1.30 times the optimal range. Table 1.1 summarizes the results in $2 \mathrm{D}$ given in Chapter 3. 


\begin{tabular}{clcl}
\hline Number of Antennae & Sum of Angles & Range (Times the Optimal Range) & Complexity \\
\hline 5 & $\varphi \geq 0$ & 1 & $O(n)$ \\
4 & $\varphi \geq 0$ & $2 \sin (\pi / 5)$ & $O(n)$ \\
3 & $\varphi \geq 0$ & $\sqrt{2}$ & $O(n)$ \\
2 & $\varphi \geq 0$ & $\sqrt{3}$ & $O(n)$ \\
2 & $\varphi<9 \pi / 20$ & 1.30 & NP-Hard \\
\hline
\end{tabular}

Table 1.1: Results of the antenna orientation problem in 2D presented in Chapter 3.

\subsubsection{Antenna Orientation Problem In 3D}

In Chapter 4 we consider the antenna orientation problem in a 3D space. The main results presented in Chapter 4 appear in the Proceedings of the 25th IEEE International Parallel $\&$ Distributed Processing Symposium (IEEE IPDPS 2011) [36].

We consider the problem when each sensor has one antenna. We present an algorithm ensuring optimal antenna range when the solid angle of each antennae is at least $\frac{18 \pi}{5}$. We also provide an algorithm for approximating the antennae range to $\sqrt{\Omega(4 \pi-\Omega)} / \pi$ times the optimal range that guarantees strong connectivity of the resulting graph when the solid angle $\Omega$ of the antennae is between $\left[2 \pi, \frac{18 \pi}{5}\right)$. We also show that when the solid angle $\Omega$ is less than $\pi$ the problem is NP-Hard. In addition, we study the effect of replacing omnidirectional antennae with directional antennae on the hop stretch factor of the resulting network of directional antennae. Thus, we present simulation results on the variation of hop stretch factor with different network sizes and solid angles of directional antennae. Table 1.2 summarizes the main results in 3D given in Chapter 4 .

\begin{tabular}{lcl}
\hline Solid Angle & Range (Times the Optimal Range) & Complexity \\
\hline$\Omega \geq 18 \pi / 5$ & 1 & Polynomial \\
$2 \pi \leq \Omega<18 \pi / 5$ & $\sqrt{\Omega(4 \pi-\Omega)} / \pi$ & Polynomial \\
$\Omega<\pi$ & 1 & NP-Hard \\
\hline
\end{tabular}

Table 1.2: Results of the antenna orientation problem in 3D with one antenna per sensor presented in Chapter 4. 


\subsubsection{Graph Orientation Problem}

Chapter 5 is devoted to the study of the graph orientation problem on 2-edge connected planar geometric graphs. The main results presented in Chapter 5 appear in the Proceedings of the Colloquium on Structural Information and Communication Complexity (SIROCCO 2010) [41].

We present three constructions for such orientations. Let $G=(V, E)$ be a connected planar graph without cut edges and let $\Phi(G)$ be the degree of the largest face in $G$. Our constructions are based on a face coloring, say with $\lambda$ colors. The first construction gives a strong orientation with at most $\left(2-\frac{4 \lambda-6}{\lambda(\lambda-1)}\right)|E|$ arcs and stretch factor at most $\Phi(G)-1$. The second construction gives a strong orientation with at most $|E|$ arcs and stretch factor at most $(\Phi(G)-1)^{\left\lceil\frac{\lambda+1}{2}\right\rceil}$. The third construction can be applied to planar graphs which are 3-edge connected. It uses a particular 6-face coloring and for any integer $k \geq 1$ produces a strong orientation with at most $\left(1-\frac{k}{10(k+1)}\right)|E|$ arcs and stretch factor at most $\Phi^{2}(G)(\Phi(G)-1)^{2 k+4}$. Table 1.3 summarizes the results presented in Chapter 5 .

\begin{tabular}{lcl}
\hline Planar Graph & Number of Arcs & Stretch Factor \\
\hline 2-Edge Connected & $\left(2-\frac{4 \lambda-6}{\lambda(\lambda-1)}\right)|E|$ & $O(\Phi(G))$ \\
2-Edge Connected & $|E|$ & $O\left(\Phi(G)^{\left\lceil\frac{\lambda+1}{2}\right\rceil}\right)$ \\
3-Edge Connected & $\left(1-\frac{1}{20}\right)|E|$ & $O\left(\Phi(G)^{8}\right)$ \\
\hline
\end{tabular}

Table 1.3: Results of the graph orientation problem presented in Chapter 5.

\subsubsection{Augmentation Problem with Bounded Length}

Chapter 6 is devoted to the study of the augmentation problem with bounded length in geometric planar graphs. We focus on augmenting the connectivity of a geometric planar graph into a 2-edge connected planar graph. The main results presented in Chapter 6 appear in the Proceedings of the 4th Annual International Conference on Combinatorial Optimization and Applications (COCOA'10) [38] and also submitted to a special issue of the journal "Discrete Mathematics, Algorithms and Applications" (DMAA).

Given a planar graph $G$ with $b$ bridges, we show how to augment $G$ with at most $b$ new edges of length bounded by three times the longest edge of $G$. If the original graph 
is a tree with $n$ nodes and max degree $\Delta$ then we prove that at most $n(1-1 / 2 \Delta)$ edges of length at most 3 are sufficient, while for MSTs at most $5 n / 6$ edges are shown to be sufficient. All these algorithms are linear in the number of vertices. We also prove that the problem of finding the minimum number of edges with bounded length is NP-Hard. In a location-aware setting (a communication model where each sensor knows its position) an algorithm is given that runs in constant time to augment a planar graph with new edges of length bounded by three times the longest edge of the original graph. We prove that this problem cannot be solved locally in a distributed manner if the nodes are locationoblivious. Table 1.4 summarizes the results presented in Chapter 6.

\begin{tabular}{lccl}
\hline Graph & Number of New Edges & Scaling Factor & Complexity \\
\hline Planar Graph with $b$ bridges & $b$ & 3 & $O(n)$ \\
Planar Tree of Max Degree $\Delta$ & $n(1-1 / 2 \Delta)$ & 3 & $O(n)$ \\
Euclidean MST & $5 n / 6$ & 3 & $O(n)$ \\
Planar Graph & Minimum & $r>0$ & NP-Complete \\
Location-Aware Setting & - & 3 & $O(1)$ \\
Location-Oblivious Setting & - & $r \geq 3$ & $O\left(\log ^{*} n\right)$ \\
\hline
\end{tabular}

Table 1.4: Results of the augmentation problem with bounded length presented in Chapter 6 .

\subsubsection{Min Degree Planar Subgraph Problem}

Chapter 7 is devoted to the answer of the minimum degree planar geometric subgraph problem. We study this problem for distinct $k$ in different settings. The main results presented in Chapter 7 appear in the Proceedings of the Algorithms and Data Structures Symposium (WADS 2011) [42].

We prove that when $k=1$ the problem is NP-Hard in qUDG and geometric graphs with orthogonal edges. However, it is trivial to solve it in a UDG. Furthermore, we prove that when $k=2$ or $k=3$, the problem is NP-hard even in the case of UDG. Tables 1.5 and 1.6 summarize the results in Chapter 7. 


\begin{tabular}{cllll}
\hline Degree & Complete graphs & UDG & $q U D G(1-\varepsilon)$ & Orthogonal graphs \\
\hline 1 & Polynomial & Polynomial & NP-Complete if $\varepsilon>0$ & NP-Complete \\
2 & Polynomial & NP-Complete & NP-Complete if $\varepsilon \geq 0$ & (open) \\
3 & Polynomial & NP-Complete & NP-Complete if $\varepsilon \geq 0$ & (open) \\
\hline
\end{tabular}

Table 1.5: Computational complexity of finding a planar spanning subgraph that has a given minimum degree in different families of geometric graphs.

\begin{tabular}{cll}
\hline Degree & Graph family & Complexity \\
\hline 1 & $U D G$ & Polynomial \\
2 & $U D G$ & NP-Complete if $\alpha<\sqrt{5} / 2$ \\
2 & $U D G$, strip of height $h$ & Polynomial if $\alpha \geq \sqrt{1+h^{2}}$ \\
3 & $U D G$ & NP-Complete if $\alpha<\sqrt{5} / 2$ \\
\hline
\end{tabular}

Table 1.6: Computational complexity of the augmentation problem.

\subsubsection{Optimal Length $k$-Connected Planar Graph Problem}

Chapter 8 is devoted to the study of the optimal length $k$-Connected planar graphs problem. We focus on 2-edge connected planar graph. The results presented in Chapter 8 appear in the paper "Approximating the Edge Length of 2-Edge Connected Planar Geometric Graphs in UDGs" [18].

We prove that given a set $S$ of $n$ points, a planar graph with minimum degree two can be constructed on $S$ with length bounded by 2 times the optimal radius. We present an algorithm for this problem that runs in $O(n \log n)$ time. We also give an algorithm with the same complexity that finds a 2-edge connected planar graph on $S$ with longest edge bounded by $\sqrt{5}$ times the optimal radius. Then, we prove that two times the optimal radius is always sufficient to construct a 2-edge connected planar graph on $S$ and we present an algorithm that runs in $O\left(n^{2}\right)$ time. Further, we show that there exist $k$-vertex connected UDGs that do not have underlying two-edge connected planar graphs even for a scaling factor of $17 / 16$ where $k \in O(\sqrt{n})$. Table 1.7 summarizes the results presented in Chapter 8 .

\subsection{Publications}

This thesis is based on the following papers. 


\begin{tabular}{llcl}
\hline$U D G$ & Planar Graph & Scaling & Complexity \\
\hline Min Degree Two & Min Degree Two & 2 & $O(n \log n)$ \\
Min Degree Two & 2-Edge Connected & $\sqrt{5}$ & $O(n \log n)$ \\
Two-Edge Connected & 2-Edge Connected & 2 & $O\left(n^{2}\right)$ \\
$k$-connected & 2-Edge Connected & $17 / 16$ & (open) \\
\hline
\end{tabular}

Table 1.7: Results of the optimal length $k$-Connected planar graphs problem presented in Chapter 8.

\section{Book Chapters}

- E. Kranakis, D. Krizanc, and O. Morales. Maintaining connectivity in sensor networks using directional antennae. In S. Nikoletseas and J. Rolim, editors, Theoretical aspects of Distributed Computing in Sensor Networks, chapter 3, pages 59-84. Springer, 2010. ISBN 978-3-642-14848-4.

\section{Refereed Conference Papers}

- E. Kranakis, O. Morales Ponce, and J. Suomela. Planar Subgraphs without LowDegree Nodes. In Proceedings of the Algorithms And Data Structures Symposium (WADS 2011), Polytechnic Institute of New York University, Brooklyn, NY, USA. Aug 15-17 2011.

- E. Kranakis, D. Krizanc, A. Modi, and O. Morales Ponce. Maintaining connectivity in 3D wireless sensor networks using directional antennae. In Proceedings of the 25th IEEE International Parallel \& Distributed Processing Symposium (IPDPS 2011), Anchorage, Alaska, USA, May 16-20 2011. IEEE Press.

- S. Dobrev, E. Kranakis, D. Krizanc, O. Morales Ponce, J. Opatrny, and L. Stacho. Strong connectivity in sensor networks with given number of directional antennae of bounded angle. In Proceedings of the 4th Annual International Conference on Combinatorial Optimization and Applications (COCOA 10). Part II, LNCS 6509, pages 72-86, Big Island, Hawaii, Dec 18-20 2010. Springer-Verlag. Also submitted to a special issue of "Discrete Mathematics, Algorithms and Applications" (DMAA).

- E. Kranakis, D. Krizanc, O. Morales Ponce, and L. Stacho. Bounded length, 2-edge 
augmentation of geometric planar graphs. In Proceedings of the 4th Annual International Conference on Combinatorial Optimization and Applications (COCOA 10). Part I, LNCS 6509, pages 385-397, Big Island, Hawaii, Dec 18-20 2010. SpringerVerlag. Also submitted to a special issue of "Discrete Mathematics, Algorithms and Applications" (DMAA).

- E. Kranakis, O. Morales Ponce, and L. Stacho. Strong orientations of planar graphs with bounded stretch factor. In Proceedings of the 17th Colloquium on Structural Information and Communication Complexity (SIROCCO 2010). LNCS 6058, pages 224-236, Sirince, Turkey, Jun 7-11 2010. Springer-Verlag.

\section{Submitted Papers}

- S. Dobrev, E. Kranakis, D. Krizanc, O. Morales Ponce, and L. Stacho. Approximating the Edge Length of 2-Edge Connected Planar Geometric Graphs in UDGs. 


\section{Chapter 2}

\section{Related Work}

\subsection{Antenna Orientation Problem}

The antenna orientation problem in 2D was first proposed by Caragiannis et al. [10]. They consider the case when each sensor has one antenna. They present polynomial time algorithms for the antenna orientation problem for the case when the sector angle of the antennae is at least $8 \pi / 5$. For smaller sector angles, they present an algorithm that approximates the minimum radius. When the sector angle is smaller than $2 \pi / 3$, they show that the problem of determining the minimum radius in order to achieve strong connectivity is NP-Hard. Later, Bhattacharya et al. [7] considered the case when each sensor has multiple (fixed number of) directional antennae and the sum of the angles is minimized. The authors presented trade-offs between antennae range and specified sums of antennae per sensor. The results presented in Chapter 3 differ from [7] in the fact that we provide algorithms to achieve the problem for angle zero, i.e., for any given angle rather than the sum of angles. Table 2.1 summarizes the results in [10] and [7].

\begin{tabular}{|c|c|c|c|}
\hline$\#$ & Sum of Angles & Times the Optimal & Paper \\
\hline 1 & $\alpha \geq 0$ & 2 & {$[54]$} \\
1 & $\pi \leq \alpha<8 \pi / 5$ & $2 \sin (\alpha / 2)$ & {$[10]$} \\
1 & $\alpha \geq 8 \pi / 5$ & 1 & {$[10]$} \\
\hline 2 & $2 \pi / 3 \leq \alpha<\pi$ & $2 \cos (\alpha / 4)$ & {$[7]$} \\
2 & $\alpha \geq \pi$ & $2 \sin (2 \pi / 9)$ & {$[7]$} \\
2 & $\alpha \geq 6 \pi / 5$ & 1 & {$[7]$} \\
\hline 3 & $\alpha \geq 0$ & $\sqrt{3}$ & {$[7]$} \\
3 & $\alpha \geq 4 \pi / 5$ & 1 & {$[7]$} \\
\hline 4 & $\alpha \geq 0$ & $\sqrt{2}$ & {$[7]$} \\
4 & $\alpha \geq 2 \pi / 5$ & 1 & {$[7]$} \\
\hline 5 & $\alpha \geq 0$ & 1 & {$[7]$} \\
\hline
\end{tabular}

Table 2.1: Results given in [10] and [7]. 
When each sensor has one antenna and the angle $\varphi=0$ then our problem is equivalent to finding a Hamiltonian cycle that minimizes the maximum length of an edge. For a set of $n$ points $1,2, \ldots, n$ with associated weights $c(i, j)$ satisfying the triangle inequality, the Bottleneck Traveling Salesman Problem (BTSP) is the min-max Hamiltonian cycle problem concerned with finding a Hamiltonian cycle for the complete graph which minimizes the maximum weight of an edge, i.e., $\min \left\{\max _{(i, j) \in H} c(i, j): H\right.$ is a Hamiltonian cycle $\}$. [54] shows that no polynomial time $(2-\varepsilon)$-approximation algorithm is possible for BTSP unless $P=N P$, and also gives a 2-approximation algorithm for this problem.

Recently, Mirela and Flatland [16] studied a slight variation of the antenna orientation problem in which they restrict the resulting graph to be a hop $s$-spanner. They consider the case when each sensor has one antenna. They fix the angles to $\pi / 2$ and $2 \pi / 3$ and prove that radius of 7 and 5 are always sufficient to establish a hop 6-spanner and hop 5-spanner respectively.

No literature is known on the connection between the MST of a set of points and strongly connected geometric spanning graph with given out-degree. Two papers relating somewhat these two concepts are the following. First, [22] shows that it is an NP-Hard problem to decide for a given set $S$ of $n$ points in the Euclidean plane and a given real parameter $k$, whether $S$ admits a spanning tree of maximum vertex degree four whose sum of edge lengths does not exceed $k$. Second, [35] gives a simple algorithm to find a spanning tree that simultaneously approximates a shortest-path tree and a minimum spanning tree. More specifically, given the two trees and a $\gamma>0$, the algorithm returns a spanning tree in which the distance between any vertex and the root of the shortest-path tree is at most $1+\gamma \sqrt{2}$ times the shortest-path distance, and the total weight of the tree is at most $1+\gamma \sqrt{2}$ times the weight of a minimum spanning tree.

The antenna orientation problem in 3D has never been studied before.

\subsection{Graph Orientation Problem}

The graph orientation problem is closely related to the antenna orientation problem. See Section 2.1 for related work. However, a similar problem that has been addressed in the literature is one that studies connectivity requirements on undirected graph that will guarantee highest edge connectivity of its orientation, c.f. [23] and [52]. 


\subsection{Augmentation Problem with Bounded Length}

Augmenting a graph into a 2-edge connected graph as well as weighted versions (shown to be NP-Complete) can be found in [21]. For any integer $k>1$, [30] and [72] give an algorithm for the minimum number of edges for augmenting any graph $G$ to a $k$-edge connected graph in polynomial time.

A similar problem is to find the minimum $k$-edge ( $k$-vertex) connected subgraph of a given $k$-edge ( $k$-vertex) connected graph, i.e., edge deletion problem. In [11] it is proved that given a 2-edge connected graph there is an algorithm running in time $O(m n)$ which finds a 2-edge connected spanning subgraph whose number of edges is $17 / 12$ times the optimal, where $m$ is the number of edges and $n$ the number of vertices of the graph. An improvement is provided in [71] in which a $4 / 3$ approximation algorithm is given. Later, Jothi et al. [32] provided a 5/4-approximation algorithm. However in these papers the resulting spanning subgraphs are not guaranteed to be planar.

If we restrict our attention to planar graphs then determining the minimum number of edges required to augment a given planar graph into a 2-vertex connected planar graph is NP-Hard [34]. Rutter and Wolf [61] proved that it is also NP-Hard to augment a geometric planar graph to a 2-edge connected geometric planar with the minimum number of new straight line edges (but the lengths of the newly added edges are unbounded). Also [33] considers the case of outerplanar graphs. Planar augmentation results for geometric graphs can be found in [1]. They show that $2 n / 3$ additional edges are required in some cases and $6 n / 7$ edges are always sufficient for augmenting a planar graph into a 2-edge connected planar graph. For the case of trees these bounds become $n / 2$ and $2 n / 3$, respectively. Although the planar graphs constructed in [1] are geometric the edge lengths of the new edges are not bounded. Similarly, Toth [68] improves the bound on the number of necessary edges for the case of two-edge connectivity.

Three connectivity in geometrical settings was studied in [69] in which they characterize geometric graphs that admit being augmented to three-edge connected and proposed an algorithm. Later Al-Jubeh et al. [3] gave a tight upper bound on the number of additional edges to augment a geometric planar graph into three-edge connected planar graph. 


\subsection{Min Degree Planar Subgraph Problem}

To our knowledge, this problem has not been studied before, but there are related problems that have been considered in prior work.

In a non-geometric setting (i.e., the embedding of the nodes is not fixed), finding a planar spanning subgraph is trivial: any spanning tree is planar. However, finding large planar subgraphs is hard. For example, deciding if there is a planar subgraph with a certain number of edges is a classical NP-Complete problem [26, problem GT27]. We refer to the survey by Liebers [49] for many variants of the theme.

Our focus is on a geometric setting which makes the problem no longer trivial: for example, an arbitrary spanning tree may have crossing edges. However, in unit disk graphs the problem is easy to solve: the Euclidean minimum spanning tree is planar. Indeed, a spanning tree is also connected, and many algorithms have been proposed to obtain a connected planar spanning subgraph of a unit disk graph, see, e.g., Gabriel and Sokal [24] and Toussaint [70]. However, none of these algorithms guarantees that the minimum degree is greater than one, and more general settings such as quasi unit disk graphs have not been extensively studied. We can also relate this problem to the augmentation problem; see Section 2.3 .

\subsection{Optimal Length $k$-Connected Planar Graph Problem}

To our knowledge, the problem for $k \geq 2$ has never been studied before. However, it is closely related to the augmentation problem and minimum degree planar subgraph problem; see Section 2.3 and Section 2.4 for related work. When the starting point is a set of points in the plane, then there exist two related papers: First, Parker [54] shows how to create a Hamiltonian cycle with length bounded by 2 times the optimal. Second, García et al. [25] show how to create a three-connected geometric planar graph with the minimum number of straight line edges of unbounded length.

The edge deletion problem was considered in the context of UDGs in [19]. They describe two simple algorithms that find subgraphs with maximal node degree of 10 and 6 that ensure both 2-edge and 2-vertex connectivity, respectively. However the resulting graphs are not planar. 


\section{Chapter 3}

\section{Antenna Orientations}

\subsection{Introduction}

Consider a set $S$ of $n$ sensors in the plane. Let $k, 1 \leq k \leq 5$ be an integer, and $\varphi, 0 \leq \varphi \leq 2 \pi$ an angle. Each sensor is equipped with $k$ directional antennae with a given transmission angle $\varphi$. We are interested in the problem of providing an algorithm for orienting the antennae at each sensor, and estimating the value of transmission range so that we obtain a strongly connected graph which spans all the sensors. We assume that the reception of each sensor is omnidirectional.

\subsubsection{Preliminaries and Notation}

Given $k$ antennae with sum of transmission angle at most $\varphi$ at each sensor. Let $r_{k}(S, \varphi)$ denote the optimal range necessary to create a strongly connected network (or spanning graph) on $S$. A special case of this is when the angle $\varphi=0$, i.e. there is a direct line connection, in which case we use the simpler notation $r_{k}(S)=r_{k}(S, 0)$.

It is useful to relate $r_{k}(S, \varphi)$ to another quantity which arises from a Minimum Spanning Tree (MST) on $S$. Let $M S T(S)$ denote the set of all MSTs on $S$. For $T \in M S T(S)$ let $r(T)$ denote the length of longest edge of $T$, and let $r_{M S T}(S)=\min \{r(T): T \in M S T(S)\}$. Clearly, for any angle $\varphi \geq 0$ we have that $r_{M S T}(S) \leq r_{k}(S, \varphi)$, since every strongly connected, directed graph on $S$ has an underlying spanning tree.

The Euclidean distance between two points is defined as $d(\cdot, \cdot) . D(u ; r)$ denotes the open disk with radius $r$, centered at $u$, and $C(u, r)$ is the circle with radius $r$ and centered at $u$. 


\subsubsection{Our Results}

We are interested in estimating the value of $r_{k}(S, \varphi)$. The optimality of antennae ranges will be compared to $r_{M S T}(S)$ called here the optimal, and without loss of generality $r_{M S T}(S)$ will be normalized, i.e., $r_{M S T}(S)=1$. The two main results in this chapter are the following.

Theorem 4. Consider a set $S$ of $n$ sensors in the plane and suppose each sensor has

$k, 1 \leq k \leq 5$, directional antennae with transmission angle $\varphi \geq 0$. If the range of each antenna is at least $2 \cdot \sin \left(\frac{\pi}{k+1}\right)$ times the optimal, then the antennae can be oriented at each sensor so that the resulting spanning digraph is strongly connected. Moreover, given an MST on the set of points $S$, such orientation can be constructed with additional $O(n)$ overhead.

Note that the case $k=1$ was derived in [54], and the case $k=5$ follows trivially since there always exists an Euclidean MST of degree five on $S$ [51]. The case when $k>5$ is optimally solved using the solution for 5 antennae and arbitrarily orienting the remaining antennae.

Theorem 5. For two antennae and angular sum of the antennae at most $\alpha$, it is NP-Hard to approximate the optimal range to within a factor of $x$, where $x$ and $\alpha$ are the solutions of equations $x=2 \sin (\alpha)$ and $x=1+2 \cos (2 \alpha)$.

Using the identity $\cos (2 \alpha)=1-2 \sin ^{2} \alpha$ and solving the resulting quadratic equation we obtain numerical values $x \approx 1.30, \alpha \approx 0.45 \pi$.

The proof of the first theorem is given in Section 3.2, and due to its length we split it into three parts. In Subsections 3.2.1,3.2.2, and 3.2.3 we deal with the case $k=4, k=3$ and $k=2$ as Theorems 8,9 , and 10 , respectively.

The pseudocode of Algorithm 1 that constructs a strongly connected spanning graph with max out-degree $2 \leq k \leq 5$, and range bounded by $2 \cdot \sin \left(\frac{\pi}{k+1}\right)$ times the optimal range, is presented in Subsection 3.2.4.

Section 3.3 contains the proof of Theorem 5 .

\subsection{Upper Bound Results on Strongly Connected Spanning Digraphs}

We begin by introducing some notation which is specific to the subsequent proofs. 
We say that two neighbours of a vertex $u$ are consecutive if the smaller sector they form with $u$ does not contain any other neighbour of $u$. In addition, we define below the concept of Antenna-Tree (A-Tree for short) which isolates the particular properties of an MST that we need in the course of the proofs.

Definition 6. An A-Tree is a tree T embedded in the plane satisfying the following three conditions:

(i) Its maximum degree is five.

(ii) The minimum angle among nodes with a common parent is at least $\pi / 3$.

(iii) For any vertex $u$ and any edge $\{u, v\}$ of $T$, the disk $D(v ; d(u, v))$ does not contain a vertex $w \neq v$ which is also a neighbor of $u$ in $T$.

An example of an A-Tree with 7 vertices is depicted in Figure 3.1. It is easy to prove that any MST on a set of points in the plane is also an A-Tree. Recall also that we consider normalized ranges i.e., we assume $r(T)=1$.

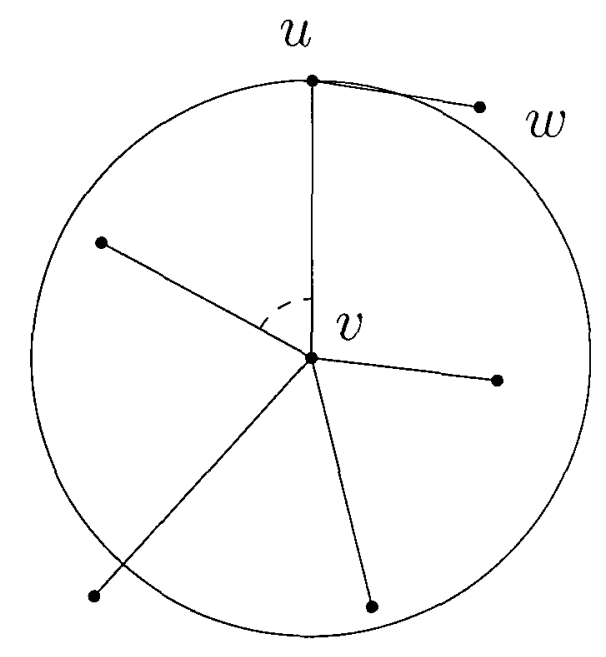

Figure 3.1: An A-Tree with 7 nodes.

Definition 7. For any real $r>0$, we define the geometric $r$-th power of a $G$, denoted by $G^{(r)}$, the graph obtained from $G$ by adding all edges between vertices of (Euclidean) distance at most $r$. 
In the sequel we refer to geometric $r$-th power as $r$-th power, for simplicity. Recall that an orientation $\vec{G}$ of a graph $G$ is a digraph obtained from $G$ by orienting edges of $G$. $d_{\vec{G}}^{+}(u)$ denotes the out-degree of $u$ in $\vec{G}$ and $\Delta^{+}(\vec{G})$ denotes the maximum over out-degrees of vertices in $\vec{G}$.

\subsubsection{Maximum Out-Degree 4}

Theorem 8. Let $T$ be an A-Tree. Then there exists a spanning graph $G \subseteq T^{\left(2 \sin \left(\frac{\pi}{5}\right)\right)}$ and its orientation $\vec{G}$ so that $\vec{G}$ is strongly connected and $\Delta^{+}(\vec{G}) \leq 4$. Moreover, $d_{\vec{G}}^{+}(u) \leq 1$ for each leaf $u$ of $T$ and every edge of $T$ incident to a leaf is contained in $G$.

Proof. We first introduce a definition used in this proof. We say that two consecutive neighbors of a vertex are close if the smaller angle they form with their common vertex is at most $2 \pi / 5$. Observe that if $v$ and $w$ are close, then $d(v, w) \leq 2 \sin (\pi / 5)$.

Let $l$ be the diameter of $T$. The proof is done by induction on the diameter of the tree. First, we do the base case for $l \leq 2$. If $l \leq 1$, let $G=T$ and the result follows trivially. If $l=2$, then $T$ is an A-Tree which is a star with $2 \leq d \leq 5$ leaves. Two cases can occur:

(i) $d<5$. Let $G=T$ and orient every edge in both directions. This results in a strongly connected digraph which trivially satisfies the hypothesis of the theorem.

(ii) $d=5$. Let $u$ be the center of $T$. Two consecutive neighbors of $u$, say, $v$ and $w$ must be close. Let $G=T \cup\{\{v, w\}\}$ and orient edges of $G$ as depicted in Figure 3.2. It is easy to check that $G$ satisfies the hypothesis of the theorem.

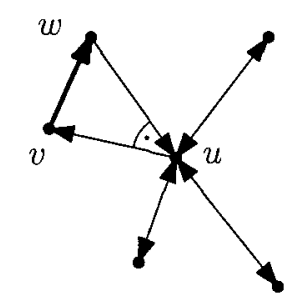

Figure 3.2: $T$ is a tree with five leaves and diameter $l=2$. (The angular sign with a dot depicts an angle of size at most $2 \pi / 5$.)

Next we continue with the inductive step. Assume $l \geq 3$ and that the theorem is valid for any A-Tree of diameter $<l$. Let $T$ be an A-Tree of diameter $l$. Consider $T^{\prime}$, the tree 
obtained from $T$ by removing all leaves. Since the removal of leaves does not violate the property of being an A-Tree, $T^{\prime}$ is also an A-Tree and has diameter less than the diameter of $T$. Thus, by inductive hypothesis, there exists $G^{\prime} \subseteq T^{\prime\left(2 \sin \left(\frac{\pi}{5}\right)\right)}$ and its orientation $\overrightarrow{G^{\prime}}$ which is strongly connected, and $\Delta^{+}\left(\overrightarrow{G^{\prime}}\right) \leq 4$. Moreover, $d_{\overrightarrow{G^{\prime}}}^{+}(u) \leq 1$ for each leaf $u$ of $T^{\prime}$ and every edge of $T^{\prime}$ incident to a leaf is contained in $G^{\prime}$.

Now we add all the removed leaves back to $T$ and construct $G$ from $G^{\prime}$ as well as corresponding orientation $\vec{G}$. We will add all removed vertices at once for each leaf $u$ of $T^{\prime}$. We describe this process only for fixed $u$. By the way we modify $G^{\prime}$ and since the diameter of $T$ is at least three, all these modifications are independent so well defined. After we add all removed vertices the resulting graph $G$ will be a spanning subgraph of $T^{\left(2 \sin \left(\frac{\pi}{5}\right)\right)}$ and its orientation $\vec{G}$ will have all the required properties. Following is the required modification for a fixed leaf $u$ of $T^{\prime}$. Let $u_{0}$ be the neighbor of $u$ in $T^{\prime}$ and $u_{1}, . ., u_{c}$ be the $c$ neighbors of $u$ in $T \backslash T^{\prime}$ in clockwise order around $u$ starting from $u_{0}$. Two cases can occur:

(i) $c \leq 3$. Let $G=G^{\prime} \cup\left\{\left\{u, u_{1}\right\}, . .,\left\{u, u_{c}\right\}\right\}$ and orient these $c$ edges in both directions thus obtaining $\vec{G}$. The graph $G \subseteq T^{\left(2 \sin \left(\frac{\pi}{5}\right)\right)}, \Delta^{+}(\vec{G}) \leq 4, d_{\vec{G}}^{+}(x) \leq 1$ for each leaf $x$ adjacent to $u$ in $T$, and every edge of $T$ joining $u$ and a leaf 1 s contaned in $G$.

(ii) $c=4$. We consider two cases. In the first case suppose that two consecutive neighbors of $u$ in $T \backslash T^{\prime}$ are close. Consider that $u_{J}$ and $u_{J+1}$ are close; where $1 \leq j<4$. Define $G=G^{\prime} \cup\left\{\left\{u, u_{1}\right\},\left\{u, u_{2}\right\},\left\{u, u_{3}\right\},\left\{u, u_{4}\right\},\left\{u_{J}, u_{j+1}\right\}\right\}$ and orient edges of $G$ as depicted in Figure 3.3a.

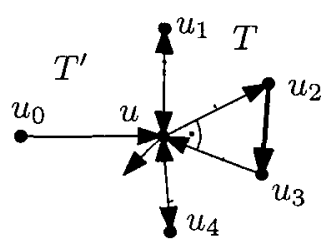

(a) $u_{J}=u_{2}$ and $u_{J+1}=$ $u_{3}$ are close

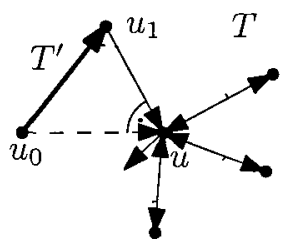

(b) $u_{0}$ and $u_{1}$ are close and $\left(u_{0}, u\right)$ is in the or1entation of $G^{\prime}$

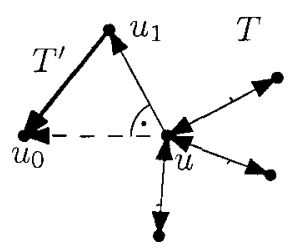

(c) $u_{0}$ and $u_{1}$ are close and $\left(u, u_{0}\right)$ is in the or1entation of $G^{\prime}$

Figure 3.3: Depicting the inductive step when $u$ has four neighbors in $T \backslash T^{\prime}$. (The dashed edge $\left\{u_{0}, u\right\}$ indicates that it does not exist in $G$ but exists in $G^{\prime}$, the angular sign with a dot depicts an angle of size at most $2 \pi / 5$, and the dotted curve is used to separate $T^{\prime}$ from T.) 
In the second case, either $u_{0}$ and $u_{1}$ are close, or $u_{0}$ and $u_{4}$ are close. Without loss of generality assume that $u_{0}$ and $u_{1}$ are close. Let

$$
G=\left\{G^{\prime} \backslash\left\{u, u_{0}\right\}\right\} \cup\left\{\left\{u, u_{1}\right\},\left\{u, u_{2}\right\},\left\{u, u_{3}\right\},\left\{u, u_{4}\right\},\left\{u_{0}, u_{1}\right\}\right\},
$$

but now the orientation of $G$ will depend on the orientation of $\left\{u, u_{0}\right\}$ in $G^{\prime}$. Thus, if $\left(u_{0}, u\right)$ is in $\overrightarrow{G^{\prime}}$, then orient edges of $G$ as depicted in Figure 3.3b, otherwise orient edges of $G$ as depicted in Figure 3.3c. The graph $G \subseteq T^{\left(2 \sin \left(\frac{\pi}{5}\right)\right)}, \Delta^{+}(\vec{G}) \leq 4$, $d_{\vec{G}}^{+}(x) \leq 1$ for each leaf $x$ adjacent to $u$ in $T$ and every edge of $T$ incident to $u$ and a leaf is contained in $G$.

This completes the proof of the theorem.

\subsubsection{Maximum Out-Degree 3}

Theorem 9. Let $T$ be an A-Tree. Then there exists a spanning graph $G \subseteq T^{(\sqrt{2})}$ and its orientation $\vec{G}$ which is strongly connected and $\Delta^{+}(\vec{G}) \leq 3$. Moreover, $d_{\vec{G}}^{+}(u) \leq 1$ for each leaf $u$ of $T$ and every edge of $T$ incident to a leaf is contained in $G$.

Proof. In this proof we say that two consecutive neighbors of a vertcx are close if the smaller angle they form with their common vertex is at most $\pi / 2$. Otherwise we say that they are far. Observe that if $v$ and $w$ are close, then $d(v, w) \leq \sqrt{2}$.

The proof is by induction on the diameter $l$ of $T$. First, we do the base case for $l \leq 2$. If $l \leq 1$, let $G=T$ and the result follows trivially. If $l=2$, then $T$ is an A-Tree which is a star with $2 \leq d \leq 5$ leaves, respectively. Three cases can occur:

(i) $d<4$. Let $G=T$ and orient every edge in both directions. This results in a strongly connected digraph which trivially satisfies the hypothesis of the theorem.

(ii) $d=4$. Let $u$ be the center of $T$. Since $T$ is a star, two consecutive neighbors of $u$, say, $u_{1}$ and $u_{2}$ are close. Let $G=T \cup\left\{\left\{u_{1}, u_{2}\right\}\right\}$ and orient edges of $G$ as depicted in Figure 3.4a. It is easy to check that $\vec{G}$ satisfies the hypothesis of the theorem.

(iii) $d=5$. Let $u$ be the center of $T$ and $u_{1}, u_{2}, u_{3}, u_{4}, u_{5}$ be the five consecutive neighbors of $u$ in clockwise order around $u$ starting at any arbitrary neighbor of $u$. Observe that at most two consecutive neighbors of $u$ are far since $T$ is a star and the angle between 
two nodes with a common parent is at least $\pi / 3$. Assume without loss of generality that $u_{5}$ and $u_{1}$ are far. Let $G=T \cup\left\{\left\{u_{1}, u_{2}\right\},\left\{u_{3}, u_{4}\right\}\right\}$ and orient edges of $G$ as depicted in Figure $3.4 \mathrm{~b}$. Thus, $\vec{G}$ satisfies trivially the hypothesis of the theorem.

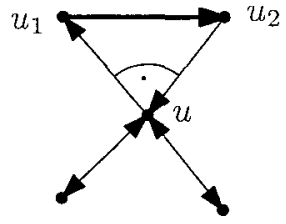

(a) $T$ has four leaves

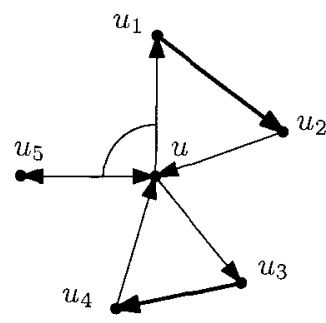

(b) $T$ has five leaves

Figure 3.4: $T$ is a tree of diameter $l=2$. (The angular sign with a dot depicts an angle of size at most $\pi / 2$ and the angular sign depicts an angle of size greater than $\pi / 2$.)

Next we continue with the inductive step. Assume $l \geq 3$ and that the theorem is valid for any A-Tree of diameter $<l$. Let $T$ be an A-Tree of diameter $l$. Consider $T^{\prime}$, the tree obtained from $T$ by removing all leaves. Since removal of leaves does not violate the property of being an A-Tree, $T^{\prime}$ is also an A-Tree and has diameter less than $l$. Thus, by inductive hypothesis there exists $G^{\prime} \subseteq T^{\prime(\sqrt{2})}$ and its orientation $\vec{G}^{\prime}$ which is strongly connected, $\Delta^{+}\left(\overrightarrow{G^{\prime}}\right) \leq 3$. Moreover, $d_{\vec{G}^{\prime}}^{+}(u) \leq 1$ for each leaf $u$ of $T^{\prime}$ and every edge of $T^{\prime}$ incident to a leaf is contained in $G^{\prime}$.

Now we add all the removed leaves back to $\mathrm{T}$ and construct $G$ from $G^{\prime}$ as well as corresponding orientation $\vec{G}$. We will add all removed vertices at once for each leaf $u$ of $T^{\prime}$. As before, we describe this process only for fixed $u$. By the way we modify $G^{\prime}$ and since the diameter of $T$ is at least three, all these modifications are independent so well defined. After we add all removed vertices the resulting graph $G$ will be a spanning subgraph of $T^{(\sqrt{2})}$ and its orientation $\vec{G}$ will have all the required properties. Following is the required modification for a fixed leaf $u$ of $T^{\prime}$. Let $u$ be a leaf of $T^{\prime}, u_{0}$ be the neighbor of $u$ in $T^{\prime}$ and $u_{1}, \ldots u_{c}$ be the $c$ neighbors of $u$ in $T \backslash T^{\prime}$ in clockwise order around $u$ starting from $u_{0}$. Three cases can occur:

(i) $u$ has at most two neighbors in $T \backslash T^{\prime}$. Let $G=G^{\prime} \cup\left\{\left\{u, u_{1}\right\},\left\{u, u_{2}\right\}\right\}$ and orient these $c$ edges in both directions. The graph $G \subseteq T^{(\sqrt{2})}, \Delta^{+}(\vec{G}) \leq 3, d_{\vec{G}}^{+}(x) \leq 1$ for 
each leaf $x$ adjacent to $u$ in $T$, and every edge of $T$ joining $u$ and a leaf is contained in $G$.

(ii) $u$ has three neighbors in $T \backslash T^{\prime}$. We consider two cases. In the first case suppose that two consecutive neighbors of $u$ in $T \backslash T^{\prime}$ are close. Assume that $u_{\jmath}$ and $u_{\jmath+1}$ are close; where $1 \leq j<3$. Let $G=G^{\prime} \cup\left\{\left\{u, u_{1}\right\},\left\{u, u_{2}\right\},\left\{u, u_{3}\right\},\left\{u_{j}, u_{j+1}\right\}\right\}$ and orient edges of $G$ as depicted in Figure 3.5a.

In the second case, either $u_{0}$ and $u_{1}$ are close or $u_{0}$ and $u_{3}$ are close. Without loss of generality assume that $u_{0}$ and $u_{1}$ are close. Thus, let $G=\left\{G^{\prime} \backslash\left\{u, u_{0}\right\}\right\} \cup$ $\left\{\left\{u, u_{1}\right\},\left\{u, u_{2}\right\},\left\{u, u_{3}\right\},\left\{u_{0}, u_{1}\right\}\right\}$. Now the orientation of $G$ will depend on the orientation of $\left\{u, u_{0}\right\}$ in $G^{\prime}$. Thus, if $\left(u_{0}, u\right)$ is in $\vec{G}^{\prime}$, then orient edges of $G$ as depicted in Figure 3.5b. Otherwise orient edges of $G$ as depicted in Figure 3.5c. The graph $G \subseteq T^{(\sqrt{2})}, \Delta^{+}(\vec{G}) \leq 3, d_{\vec{G}}^{+}(x) \leq 1$ for each leaf $x$ of $T$ incident to $u$, and every edge of $T$ joining $u$ and a leaf is contained in $G$.

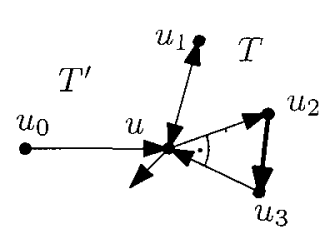

(a) $u_{l}=u_{2}$ and $u_{l+1}=$ $u_{3}$ are close

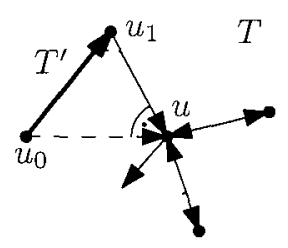

(b) $u_{0}$ and $u_{1}$ are close and $\left(u_{0}, u\right)$ is in the or1entation of $G^{\prime}$

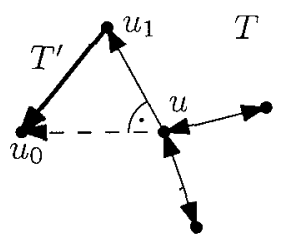

(c) $u_{0}$ and $u_{1}$ are close $\left(u, u_{0}\right)$ is in the orientation of $G^{\prime}$

Figure 3.5: Depicting the inductive step when $u$ has three neighbors in $T \backslash T^{\prime}$. (The dashed edge $\left\{u, u_{0}\right\}$ indicates that it does not exist in $G$ but exists in $G^{\prime}$, the angular sign with a dot depicts an angle of size at most $\pi / 2$ and the dotted curve is used to separate $T^{\prime}$ from T.)

(iii) $u$ has four neighbors in $T \backslash T^{\prime}$. We consider two cases. In the first case suppose that either $u_{0}$ and $u_{1}$ are far or $u_{2}$ and $u_{3}$ are far or $u_{4}$ and $u_{0}$ are far. Assume without loss of generality that $u_{0}$ and $u_{1}$ are far. Let

$$
G=G^{\prime} \cup\left\{\left\{u, u_{1}\right\},\left\{u, u_{2}\right\},\left\{u, u_{3}\right\},\left\{u, u_{4}\right\},\left\{u_{1}, u_{2}\right\},\left\{u_{3}, u_{4}\right\}\right\}
$$

and orient edges of $G$ as depicted in Figure 3.6a.

In the second case, assume either $u_{1}$ and $u_{2}$ are far or $u_{3}$ and $u_{4}$ are far. Assume without loss of generality that $u_{1}$ and $u_{2}$ are far. Let $G=\left\{G^{\prime} \backslash\left\{u, u_{0}\right\}\right\} \cup$ 
$\left\{\left\{u, u_{1}\right\},\left\{u, u_{2}\right\},\left\{u, u_{3}\right\},\left\{u, u_{4}\right\}\left\{u_{0}, u_{1}\right\},\left\{u_{2}, u_{3}\right\}\right\}$. The orientation $\vec{G}$ will depend on the orientation of $\left\{u, u_{0}\right\}$ in $G^{\prime}$. Thus, if $\left(u_{0}, u\right)$ is in $\overrightarrow{G^{\prime}}$, then orient edges of $G$ a s depicted in Figure 3.6b. Otherwise orient edges of $G$ as depicted in Figure 3.6c. The graph $G \subseteq T^{(\sqrt{2})}, \Delta^{+}(\vec{G}) \leq 3, d_{\vec{G}}^{+}(x) \leq 1$ for each leaf $x$ of $T$ adjacent to $u$, and every edge of $T$ joining $u$ and a leaf is contained in $G$.

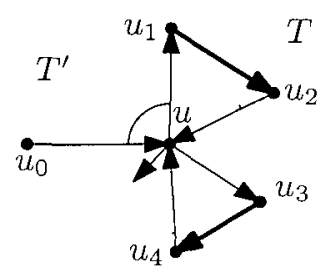

(a) $u_{0}$ and $u_{1}$ are far

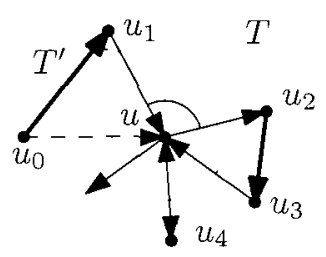

(b) $u_{1}$ and $u_{2}$ are far and $\left(u_{0}, u\right)$ is in the orientation of $G^{\prime}$

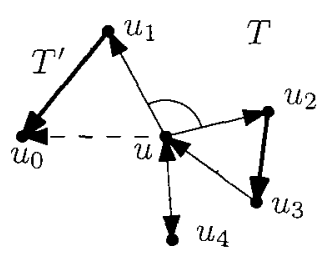

(c) $u_{1}$ and $u_{2}$ are far and $\left(u, u_{0}\right)$ is in the orientation of $G^{\prime}$

Figure 3.6: Depicting the inductive step when $u$ has four neighbors in $T \backslash T^{\prime}$. (The dashed edge $\left\{u_{0}, u\right\}$ indicates that it does not exist in $G$ but exits in $G^{\prime}$, the angular sign depicts an angle of size greater than $\pi / 2$ and the dotted curve is used to separate the tree $T^{\prime}$ from $T$.)

This completes the proof of the theorem.

\subsubsection{Maximum Out-Degree 2}

Theorem 10. Given an A-Tree $T$, there exists a spanning graph $G \subseteq T^{(\sqrt{3})}$ and tts orientation $\vec{G}$ which is strongly connected and $\Delta^{+}(\vec{G}) \leq 2$. Moreover, for each leaf $u$ of $T, d_{\vec{G}}^{+}(u) \leq 1$, and either the edge incident to $u$ is in $G$ or $u$ has two other siblings (one ummediately preceding it and other immediately following it in the embedding of $T$ ) and $u$ is adjacent to both in $G$.

Before proving Theorem 10, we need to introduce a definition and two lemmas which provide information on the proximity of two vertices with a common parent.

In the rest of this section we say that two neighbors of a vertex are close if the distance between them is at most $\sqrt{3}$. Otherwise we say that they are far.

Lemma 11. Let $u$ and $v$ be two consecutive siblings in an A-Tree with common parent $p$ such that the angle $\alpha=\angle(u p v) \leq 2 \pi / 3$ and $v$ is at distance one from $p$. Then, a chuld $v^{\prime}$ 
of $v$ with angle $\angle\left(p v v^{\prime}\right) \leq \gamma$ is close to $u$, where:

$$
\gamma= \begin{cases}\frac{5 \pi}{3}-2 \alpha & \text { if } \frac{\pi}{3} \leq \alpha \leq \frac{\pi}{2} \\ \frac{2 \pi}{3} & \text { if } \frac{\pi}{2}<\alpha \leq \frac{\pi}{6}+\arccos \left(\frac{1}{2 \sqrt{3}}\right) \\ \frac{5 \pi}{9} & \text { if } \frac{\pi}{6}+\arccos \left(\frac{1}{2 \sqrt{3}}\right)<\alpha \leq \frac{2 \pi}{3}\end{cases}
$$

Proof. We prove each case separately:

(i) Consider a fixed angle $\frac{\pi}{3} \leq \alpha \leq \frac{\pi}{2}$. Observe that $2 \cos (\alpha) \leq d(u, p) \leq 1$, since from definition of A-Tree, $u \notin D(v ; d(v, p))$. Consider the intersection area $I$ among all the disk of radius $\sqrt{3}$ centered at each point $u$ with angle $\angle(u p v)=\alpha$ and $2 \cos (\alpha) \leq$ $d(u, p) \leq 1$ as depicted in Figure 3.7. Observe that each neighbor of $v$ inside $I$ is close to $u$. It is sufficient to calculate the minimum angle with apex at $v$ that covers I. Observe that it is determined by $D(u ; \sqrt{3})$ where $d(u, p)=2 \cos (\alpha)$. Fix $u$ at distance $2 \cos (\alpha)$ from $p$ and angle $\angle(u p v)=\alpha$. Let $y \in C(u ; \sqrt{3}) \cap C(v ; 1)$ be the intersection point in $I$. Let $\angle(p v y)=\angle(p v u)+\angle(u v y)$. It is easy to see that $\angle(p v u)=\pi-2 \alpha$ and from the law of cosine in the triangle $u v y, \angle(u v y)=2 \pi / 3$ since $d(u, y)=\sqrt{3}$, and $d(u, v)=d(v, y)=1$. Therefore, $\angle(p v y) \leq \gamma=\frac{5 \pi}{3}-2 \alpha$.

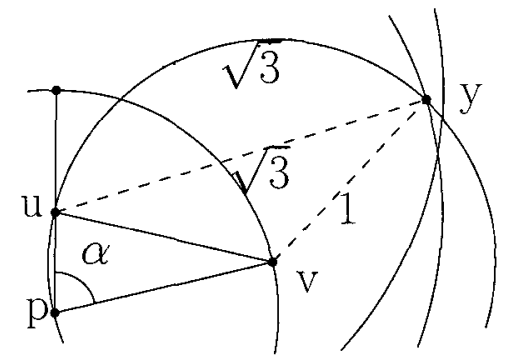

Figure 3.7: Depicting the case when $\pi / 3 \leq \alpha \leq \pi / 2$.

(ii) Consider a fixed angle $\frac{\pi}{2}<\alpha \leq \pi / 6+\arccos \left(\frac{1}{2 \sqrt{3}}\right)$. Since $\alpha>\frac{\pi}{2}, 0<d(u, p) \leq$ 1. Consider the intersection area $I$ among all the disk of radius $\sqrt{3}$ centered at each point $u$ with angle $\angle(u p v)=\alpha$ and $0<d(u, p) \leq 1$ as depicted in Figure 3.8. Observe that each neighbor of $v$ inside $I$ is close to $u$. It is sufficient to calculate the minimum angle with apex at $v$ that covers $I$. Consider $y \in C(p ; \sqrt{3}) \cap C(u, \sqrt{3})$ be the intersection near $v$ where $d(p, u)=1$ and $v^{\prime} \in C(p, 1) \cap C(y, 1)$ be the intersection point furthest from $u$. If $\alpha \leq \angle\left(u p v^{\prime}\right)$, then the minimum angle is determined by 
$D(p, \sqrt{3})$. Using the law of cosine in $u p y$ and $p v^{\prime} y, \angle\left(u p v^{\prime}\right)=\pi / 6+\arccos \left(\frac{1}{2 \sqrt{3}}\right)$ since $d(u, p)=d\left(p, v^{\prime}\right)=d\left(v^{\prime} y\right)=1$ and $d(u, u)=d(p, y)=\sqrt{3}$. Let $y \in C(p ; \sqrt{3}) \cap$ $C(v ; 1)$ be the intersection point in $I$. Hence, $\angle(p v y) \leq \gamma=2 \pi / 3$.

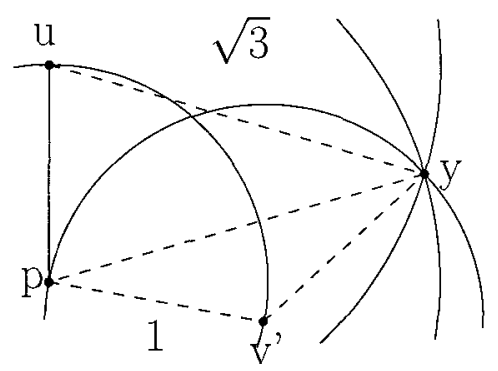

Figure 3.8: Depicting the case when $\pi / 2<\alpha \leq \pi / 6+\arccos (1 / 2 \sqrt{3})$.

(iii) Consider a fixed angle $\frac{\pi}{6}+\arccos \left(\frac{1}{2 \sqrt{3}}\right)<\alpha \leq \frac{2 \pi}{3}$. Since $\alpha>\frac{\pi}{2}, 0<d(u, p) \leq 1$. Consider the intersection area $I$ among all the disk of radius $\sqrt{3}$ centered at each point $u$ with angle $\angle u p v=\alpha$ at distance in the interval $(0,1]$ from $p$ as depicted in Figure 3.8. Observe that each neighbor of $v$ inside $I$ is close to $u$. It is sufficient to calculate the minimum angle with apex at $v$ that covers $I$. However, from the previous case, it is determined by $D(u, \sqrt{3})$ where $d(u, p)=1$. Moreover, the angle decreases when $\alpha$ increases. Therefore, the minimum angle is reached when the $\alpha=2 \pi / 3$. Thus, fix $\alpha=2 \pi / 3$. Let $y \in C(v, 1) \cap C(u, \sqrt{3})$ be the intersection point in $I$ as depicted in Figure 3.9. From the law of cosine in the triangle $u p v$, $\angle(p v u)=\pi / 6$ since $d(u, p)=d(p, v)=1$. Similarly, by law of cosine in the triangle $u v y, \angle(u v y)=\arccos \left(\frac{1}{2 \sqrt{3}}\right)$ since $d(v, y)=1$ and $d(u, y)=d(u, v)=\sqrt{3}$. Therefore, $\angle(p v y) \leq \frac{\pi}{6}+\arccos \left(\frac{1}{2 \sqrt{3}}\right)>\frac{5 \pi}{9}$.

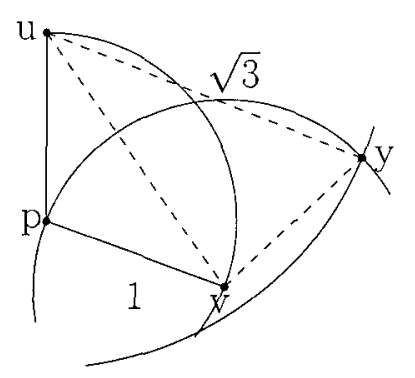

Figure 3.9: Depicting the case when $\pi / 6+\arccos (1 / 2 \sqrt{3})<\alpha \leq 2 \pi / 3$. 
Lemma 12. Let $u, v$ and $w$ be three consecutive siblings with parent $p$ in an A-Tree $T$ such that $\angle(u p v)+\angle(v p w) \leq \pi$.

(i) If $d(v)=3$ and the only two children of $v$ are far, then at least one of them is close to either $u$ or $w$.

(ii) If $d(v)=4$ and each pair of consecutive children of $v$ are close, then at least one of them is close to either $u$ or $w$.

(iii) If $d(v)=4$, two consecutive children of $v$ are far and all children of $v$ are at distance at least $\sqrt{3}-1$ of $v$, then one child of $v$ is close to $u$ and another child of $v$ is close to $w$.

(iv) If $d(v)=4$, two consecutive children of $v$ are far and one child $x$ of $v$ is at distance at most $\sqrt{3}-1$ of $v$, then at most one child of $v$ different from $x$ are far from $u$ and $w$.

(v) If $d(v)=5$, then at least one child of $v$ is close to either $u$ or $w$.

Proof. Let $\alpha=\angle(u p v)$ and $\beta=\angle(v p w)$. We first prove the particular cases when $\alpha+\beta=$ $\pi$ and $d(p, v)=1$. After that, we prove the general case when $d(p, v)<1$ and/or $\alpha+\beta<\pi$.

Without loss of generality, consider $\pi / 3 \leq \alpha \leq \pi / 2$. Let $\beta=\pi-\alpha$. Using Lemma 11 we divide the circle into three different regions: $\mathcal{C}, \mathcal{D}$ and $\mathcal{E}$ as depicted in Figure 3.10 in such a way that: $\angle C \geq \frac{5 \pi}{3}-2 \alpha$ and if $\alpha \leq 5 \pi / 6-\arccos \left(\frac{1}{2 \sqrt{3}}\right)$, then $\angle \mathcal{D} \geq \frac{5 \pi}{9}$. Otherwise, $\angle \mathcal{D}=2 \pi / 3$. Let $\angle(\mathcal{E})<2 \pi-(\angle(\mathcal{C})+\angle(\mathcal{D}))$. i.e., if $\alpha \leq 5 \pi / 6-\arccos \left(\frac{1}{2 \sqrt{3}}\right) \leq$ $\frac{13 \pi}{30}$, then $\angle(\mathcal{E})<2 \alpha-\frac{2 \pi}{9} \leq 29 \pi / 45$. Otherwise, $\angle(\mathcal{E})<\frac{2 \pi}{3}$. Observe that the neighbors of $v$ inside $C$ are close to $u$ and the neighbors of $v$ inside $\mathcal{D}$ are close to $w$ and the neighbors of $v$ inside $\mathcal{E}$ are (possibly) far from $u$ or $w$.

Let $v_{0}=p, v_{1}, \cdots, v_{c}$ the neighbors of $v$ in clockwise order. Now, we prove each case of Lemma 12.

(i) $d(v)=3$ and $v_{1}$ is far from $v_{2}$. At most one child of $v$ can be in $\mathcal{E}$, since $\angle(\mathcal{E})$ is less than $2 \pi / 3$ and $\angle\left(v_{1} v v_{2}\right) \geq 2 \pi / 3$. 


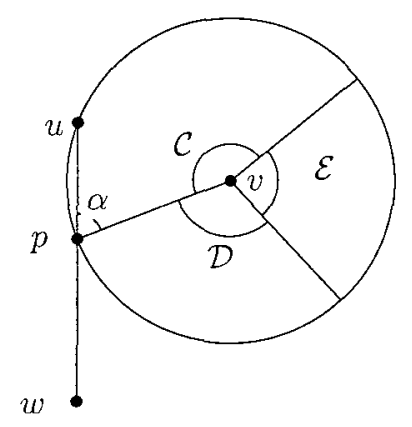

Figure 3.10: Cones $\mathcal{C}, \mathcal{D}, \mathcal{E}$ with apex at $v$.

(ii) If $d(v)=4$ and each pair of consecutive children of $v$ are close. Since the minimum distance among children is $\pi / 3$ and $\angle(\mathcal{E})<2 \pi / 3$, at most two children of $v$ can be in $\mathcal{E}$.

(iii) If $d(v)=4$, two consecutive children of $v$ are far and all children of $v$ are at distance at least $\sqrt{3}-1$ from $v$. Since two children are far, $\angle\left(v_{1} v v_{3}\right)>\pi$. Hence, when $\alpha \geq 5 \pi / 6-\arccos (1 / 2 \sqrt{3}), \angle(C) \geq 2 \pi / 3$ and $\angle(\mathcal{D})=2 \pi / 3$. Therefore, $v_{1} \in \mathcal{C}$ and $v_{3} \in \mathcal{D}$. It remains to prove the case when $\alpha<5 \pi / 6+\arccos (1 / 2 \sqrt{3})$. Assume without loss of generality that $v_{1}$ is close to $u$. From the definition of A-Tree and the hypothesis, $u \notin D\left(v_{1} ; 1\right) \cup D(v ; 1)$ and $v_{1} \notin D(v ; \sqrt{3}-1)$. Let $y \in C(u ; 1) \cap C(v ; \sqrt{3}-$ 1) be the intersection point farthest from $p$ as depicted in Figure 3.11. Therefore, $\angle\left(p v v_{1}\right) \geq \angle(p v y)=\angle(p v u)+\angle(u v y)$. We will prove that $\angle(p v y) \geq 4 \pi / 9$ and since two consecutive children are far, $\angle\left(w v v_{3}\right) \leq 2 \pi-\left(\angle\left(p v v_{1}\right)+\pi\right) \leq 5 \pi / 9$. As a consequence, $v_{3} \in \mathcal{D}$. From the law of cosine in $u v y, \angle(u v y) \geq 17 \pi / 45$ since $d(u, y) \geq 1, d(u, v) \geq 1$ and $d(v, y)=\sqrt{3}-1$. Further, $\angle(p v u) \geq \pi-2 \alpha \geq 2 \pi / 15$ since $\alpha<5 \pi / 6+\arccos (1 / 2 \sqrt{3})$ Therefore, $\angle(p v y)>4 \pi / 9$.

(iv) If $d(v)=4$, two consecutive children of $v$ are far and one child of $v$ is at distance at most $\sqrt{3}-1$ from $v$. Notice that if $v_{2}$ is at distance at most $\sqrt{3}-1$ from $v$, then $v_{1}$ is close to $v_{2}$ and $v_{2}$ is close to $v_{3}$. Therefore, $v_{2}$ is at distance at least $\sqrt{3}-1$ from $v$ and either $v_{1}$ or $v_{3}$ is at distance $\sqrt{3}-1$ from $v$. Assume without loss of generality that $v_{1}$ is at distance at most $\sqrt{3}-1$ from $v$. Therefore, $v_{2}$ is far from $v_{3}$ and only one of them can be inside $\mathcal{E}$ since $\angle(\mathcal{E})$ is less than $2 \pi / 3$.

(v) $d(v)=5$. At most two children of $v$ can be in $\mathcal{E}$, because $\angle(\mathcal{E})$ is less than $2 \pi / 3$ 


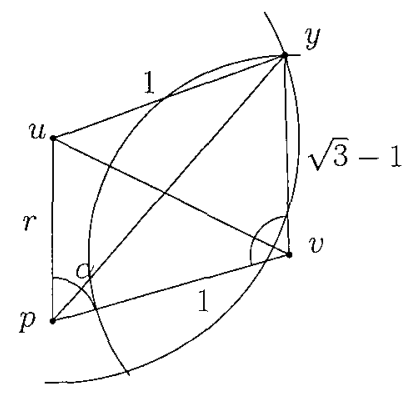

Figure 3.11: Depicting when $d(v)=4$, two consecutive children of $v$ are far and all the children are at distance at least $\sqrt{3}-1$ from $v$.

and two children are at distance at least $\pi / 3$.

This proves the case when $d(p, v)=1$. To prove the case when $d(p, v)<1$, consider the intersection point $v^{\prime}$ with $C(p, 1)$ and the ray emanating from $p$ toward $v$. Therefore, $d(p, v)<d\left(p, v^{\prime}\right)$ and $d(u, v)<d\left(u, v^{\prime}\right)$. If we move all children of $v$ toward $v^{\prime}$, the distance from $u$ to them will increase. Hence, the solution for $d(p, v)=1$ covers all cases in line segment $\overline{p, v^{\prime}}$.

Now we prove the case when $\alpha+\beta<\pi$. Consider the line segment $\overline{u, w}$ and its intersection point $p^{\prime}$ with the edge $\{p, v\}$. Notice that by replacing $p$ with $p^{\prime}$ we get $\alpha^{\prime}+\beta^{\prime}=\pi$ such that $\alpha<\alpha^{\prime}$ and $\beta<\beta^{\prime}$. Hence, the solution in the case $\alpha+\beta=\pi$ is also a solution when $\alpha+\beta<\pi$. This completes the proof of Lemma 11 .

Proof of Theorem 10. The proof is by induction on the diameter $l$ of $T$. First, we do the base case $l \leq 2$. If $l \leq 1$, let $G=T$ and the result follows trivially.

If $l=2$, then $T$ is an A-Tree which is a star with $2 \leq d \leq 5$ leaves, respectively. Four cases can occur:

(i) $d=2$. Let $G=T$ and orient every edge in both directions. This results in a strongly connected digraph which trivially satisfies the hypothesis of the theorem.

(ii) $d=3$. Let $u$ be the center of $T$. Since $T$ is a star, two consecutive neighbors, say $u_{1}$ and $u_{2}$ are close. Let $G=T \cup\left\{\left\{u_{1}, u_{2}\right\}\right\}$ and orient edges of $G$ as depicted in Figure $3.12 \mathrm{a}$. It is easy to check that $\vec{G}$ satisfies the hypothesis of the theorem. 
(iii) $d=4$. Let $u$ be the center of $T$ and $u_{1}, u_{2}, u_{3}, u_{4}$ be the four neighbors of $u$ in clockwise order around $u$ starting at any arbitrary neighbor of $u$. Observe that at most two consecutive neighbors of $u$ are far since $T$ is a star and the angle between two nodes with a common parent is at least $\pi / 3$. Assume without loss of generality that $u_{4}$ and $u_{1}$ are far. Let $G=T \cup\left\{\left\{u_{1}, u_{2}\right\},\left\{u_{3}, u_{4}\right\}\right\}$ and orient edges of $G$ as depicted in Figure 3.12 b. Thus, $\vec{G}$ satisfies trivially the hypothesis of the theorem.

(iv) $d=5$. Let $u$ be the center of $T$ and $u_{1}, u_{2}, u_{3}, u_{4}, u_{5}$ be the five neighbors of $u$ in clockwise order around $u$ starting at any arbitrary neighbor of $u$. Observe that all consecutive neighbors are close since $T$ is a star and the angle between two nodes with a common parent is at least $\pi / 3$. Let $G=T \backslash\left\{u, u_{4}\right\} \cup\left\{\left\{u_{1}, u_{2}\right\},\left\{u_{3}, u_{4}\right\},\left\{u_{4}, u_{5}\right\}\right\}$ and orient edges of $G$ as depicted in Figure 3.12c. Observe that $\angle\left(u_{3} u u_{5}\right) \leq \pi$. Orientation $\vec{G}$ is strongly connected and $\Delta^{+}(\vec{G}) \leq 2$. Moreover, $d_{\vec{G}}^{+}(u) \leq 1$, all edges of $T$ except $\left\{u, u_{4}\right\}$ are contained in $G$ and $\left\{u_{3}, u_{4}\right\}$ and $\left\{u_{4}, u_{5}\right\}$ are contained in $G$.

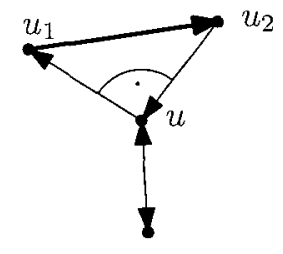

(a) $T$ has three leaves

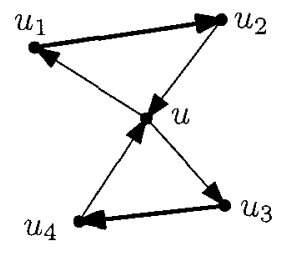

(b) $T$ has four leaves

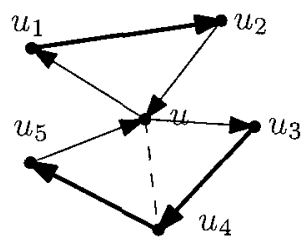

(c) $T$ has five leaves

Figure 3.12: $T$ is a tree with diameter $l=2$. (The heavy arrows represent the newly added edges, the angular sign with a dot depicts an angle of size at most $2 \pi / 3$ and dashed edge indicates that it exists in $T$ but not in $G$.)

Next we continue with the inductive step. Assume $l \geq 3$ and that the theorem is valid for any A-Tree of diameter $<l$. Let $T$ be an A-Tree of diameter $l$. Consider $T^{\prime}$, the tree obtained from $T$ by removing all leaves. Since removal of leaves does not violate the property of being an A-Tree, $T^{\prime}$ is also an A-Tree and has diameter less than $l$. Thus, by inductive hypothesis there exists $G^{\prime} \subseteq T^{\prime(\sqrt{3})}$ and its orientation $\vec{G}^{\prime}$ which is strongly connected, $\Delta^{+}\left(\overrightarrow{G^{\prime}}\right) \leq 2$. Moreover, for each leaf $u$ of $T^{\prime}, d_{\vec{G}^{\prime}}^{+}(u) \leq 1$, and either the edge incident to $u$ is in $G^{\prime}$ or $u$ has two other siblings (one immediately preceding it and other immediately following it in the embedding of $T^{\prime}$ ) and $u$ is adjacent to both in $G^{\prime}$. 
Now we add all the removed leaves back to $\mathrm{T}$ and construct $G$ from $G^{\prime}$ as well as corresponding orientation $\vec{G}$. We will add all removed vertices at once for each leaf $u$ of $T^{\prime}$. As before, we describe this process only for fixed $u$. By the way we modify $G^{\prime}$ and since the diameter of $T$ is at least three, all these modifications are independent so well defined. After we add all removed vertices the resulting graph $G$ will be a spanning subgraph of $T^{(\sqrt{3})}$ and its orientation $\vec{G}$ will have all the required properties. Following is the required modification for a fixed leaf $u$ of $T^{\prime}$. Let $u_{0}$ be the neighbor of $u$ in $T^{\prime}$ and $u_{1}, \ldots, u_{c}$ be the $c$ neighbors of $u$ in $T \backslash T^{\prime}$ in clockwise order around $u$ starting from $u_{0}$. Four cases can occur:

(i) $u$ has one neighbor in $T \backslash T^{\prime}$. Let $G=G^{\prime} \cup\left\{\left\{u, u_{1}\right\}\right\}$ and orient it in both directions. It is easy to see that $\vec{G}$ satisfies the inductive hypothesis.

(ii) $u$ has two neighbors in $T \backslash T^{\prime}$. We consider two cases. In the first case suppose that $u_{1}$ and $u_{2}$ are close. Let $G=G^{\prime} \cup\left\{\left\{u, u_{1}\right\},\left\{u, u_{2}\right\},\left\{u_{1}, u_{2}\right\}\right\}$ and orient edges of $G$ as depicted in Figure 3.13a. In the second case, $u_{1}$ and $u_{2}$ are far. Again we need to consider two cases:

(i) $\left\{u_{0}, u\right\}$ is in $G^{\prime}$. Either $u_{0}$ and $u_{1}$ are close or $u_{2}$ and $u_{0}$ are close. Without loss of generality assume that $u_{1}$ and $u_{0}$ are close. Let $G=\left\{G^{\prime} \backslash\left\{u_{0}, u\right\}\right\} \cup$ $\left\{\left\{u, u_{1}\right\},\left\{u, u_{2}\right\},\left\{u_{0}, u_{1}\right\}\right\}$. If $\left(u_{0}, u\right)$ is in $\overrightarrow{G^{\prime}}$, then orient edges of $G$ as depicted in Figure 3.13b. Otherwise orient edges of $G$ as depicted in Figure 3.13c. Thus, $\vec{G}$ is strongly connected and $\Delta^{+}(\vec{G}) \leq 2$. Moreover, the leaves $u_{1}$ and $u_{2}$ of $T$ have degree one and the edges of $T$ incident to them are contained in $G$.

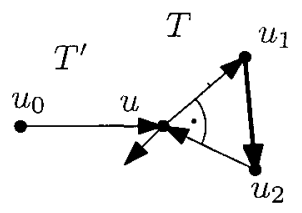

(a) $u_{1}$ and $u_{2}$ are close $\left\{u_{0}, u\right\}$

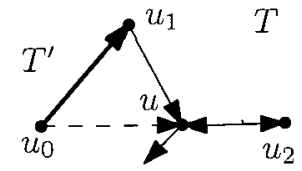

(b) $u_{0}$ and $u_{1}$ are far and $\left(u, u_{0}\right)$ is in the orientation of $G^{\prime}$

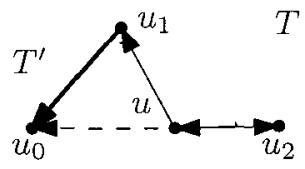

(c) $u_{0}$ and $u_{1}$ are far and $\left(u, u_{0}\right)$ is in the onentation of $G^{\prime}$

Figure 3.13: Depicting the inductive step when $u$ has two neighbors in $T \backslash T^{\prime}$. (The dashed edge $\left\{u_{0}, u\right\}$ indicates that it does not exist in $G$ but exists in $G^{\prime}$ and the dotted curve is used to separate $T^{\prime}$ from $T$.) 
(ii) $\left\{u_{0}, u\right\}$ is not in $G^{\prime}$ By inductive hypothesis, $u$ is connected to its two siblings $v$ and $w$ in $G^{\prime}$. Thus, by Lemma 12, either $u_{1}$ or $u_{2}$ are close to $v$ or $w$. Without loss of generality assume that $u_{1}$ and $v$ are close. Let $G=\left(G^{\prime} \backslash\{v, u\}\right) \cup$ $\left\{\left\{u_{1}, u\right\},\left\{u_{2}, u\right\},\left\{v, u_{1}\right\}\right\}$. If $(v, u)$ is in $\overrightarrow{G^{\prime}}$, then orient edges of $G$ as depicted in Figure 3.14a. Otherwise orient edges of $G$ as depicted in Figure 3.14b. Thus, $\vec{G}$ is strongly connected and $\Delta^{+}(\vec{G}) \leq 2$. Moreover, the leaves $u_{1}$ and $u_{2}$ of $T$ have degree one and the edges of $T$ incident to them are contained in $G$.

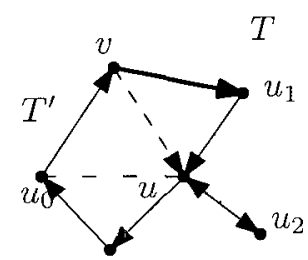

(a) $\left(u_{0}, u\right)$ is in the orientation of $G^{\prime}$

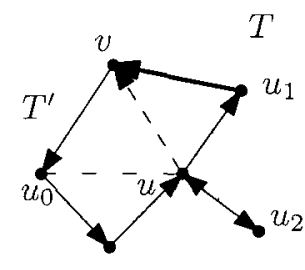

(b) $\left(u, u_{0}\right)$ is in the orrentation of $G^{\prime}$

Figure 3.14: Depicting the inductive step when $u$ has two neighbors in $T \backslash T^{\prime}, u_{0}$ and $u_{1}$ are far and $\left\{u_{0}, u\right\}$ is not in $G^{\prime}$. (The dashed edge $\{v, u\}$ indicates that it does not exist in $G$ but exists in $G^{\prime}$, the dash dotted edge $\left\{u_{0}, u\right\}$ indicates that it exists in $T^{\prime}$ but not in $G^{\prime}$ and the dotted curve is used to separate $T^{\prime}$ from $T$.)

(iii) $u$ has three neighbors in $T \backslash T^{\prime}$. Two cases can occur:

(i) $\left\{u_{0}, u\right\}$ is in $G^{\prime}$. At most two neighbors of $u$ are far. First, suppose that $u_{3}$ and $u_{0}$ are far (This case is equivalent to the case when $u_{1}$ and $u_{2}$ are far.) Let $G=\left\{G^{\prime} \backslash\left\{u_{0}, u\right\}\right\} \cup\left\{\left\{u_{1}, u\right\},\left\{u_{2}, u\right\},\left\{u_{3}, u\right\},\left\{u_{1}, u_{0}\right\},\left\{u_{2}, u_{3}\right\}\right\}$. If $\left(u_{0}, u\right)$ is in $\overrightarrow{G^{\prime}}$, then orient edges of $G$ as depicted in Figure 3.15a. Otherwise orient edges of $G$ as depicted in Figure 3.15b. Thus, $\vec{G}$ is strongly connected and $\Delta^{+}(\vec{G}) \leq 2$. Moreover, the leaves $u_{1}, u_{2}$ and $u_{3}$ of $T$ have degree one and the edges of $T$ incident to them are contained in $G$. By symmetry, we can prove the case when $u_{1}$ and $u_{0}$ are far or $u_{2}$ and $u_{3}$ are far.

(ii) $\left\{u_{0}, u\right\}$ is not $1 \mathrm{n} G^{\prime}$. By inductive hypothesis $u$ is connected to its two siblings $v$ and $w$ in $G^{\prime}$. Three cases can occur.

(i) $u_{1}$ is close to $u_{2}$ and $u_{2}$ is close to $u_{3}$. By Lemma 12 , either $u_{1}$ or $u_{3}$ is close to either $v$ or $w$. Assume that $v$ and $u_{1}$ are close. Let $G=\left\{G^{\prime} \backslash\right.$ 


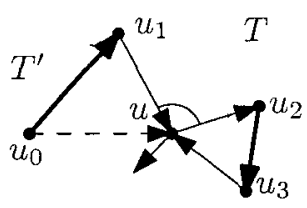

(a) $\left(u_{0}, u\right)$ is in the or1entation of $G^{\prime}$

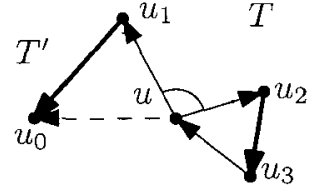

(b) $\left(u, u_{0}\right)$ is in the or1entation of $G^{\prime}$

Figure 3.15: Depicting the inductive step when $u$ has three neighbors in $T \backslash T^{\prime}, u_{1}$ and $u_{2}$ are far and $\left\{u_{0}, u\right\}$ is in $G^{\prime}$. (The dashed edge $\left\{u_{0}, u\right\}$ indicates that it does not exist in $G$ but exists in $G^{\prime}$ and the dotted curve is used to separate $T^{\prime}$ from $T$.)

$\{v, u\}\} \cup\left\{\left\{u_{1}, u\right\},\left\{u_{2}, u\right\},\left\{u_{3}, u\right\},\left\{v, u_{1}\right\},\left\{u_{2}, u_{3}\right\}\right\}$. If $(v, u)$ is in $\overrightarrow{G^{\prime}}$, then orient edges of $G$ as depicted in Figure 3.16a. Otherwise orient edges of $G$ as depicted in Figure 3.16b. Thus, $\vec{G}$ is strongly connected and $\Delta^{+}(\vec{G}) \leq 2$. Moreover, the leaves $u_{1}, u_{2}$ and $u_{3}$ of $T$ have degree one and the edges of $T$ incident to them are contained in $G$.

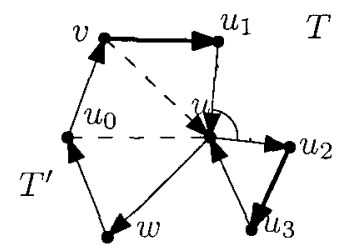

(a) $(v, u)$ is in the orientation of $G^{\prime}$

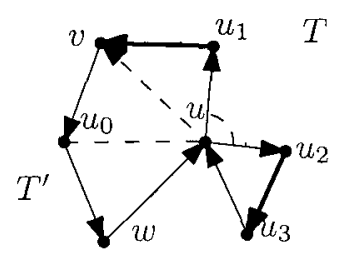

(b) $(u, v)$ is in the orientation of $G^{\prime}$

Figure 3.16: Depicting the inductive step when $u$ has three neighbors in $T \backslash T^{\prime}, u_{1}$ and $u_{2}$ are far and $\left\{u_{0}, u\right\}$ is not in $G^{\prime}$. (The dashed edge $\{v, u\}$ indicates that it does not exist in $G$ but exists in $G^{\prime}$, the dash dotted edge $\left\{u_{0}, u\right\}$ indicates that it exists in $T^{\prime}$ but not in $G^{\prime}$ and the dotted curve is used to separate $T^{\prime}$ from $T$.)

(ii) Either $u_{1}$ is far from $u_{2}$ or $u_{2}$ is far from $u_{3}$ and $u_{1}, u_{2}$ and $u_{3}$ are at distance greater than $\sqrt{3}-1$ from $u$. By Lemma $12 u_{1}$ is close to one sibling of $u$, say $v$ and $u_{3}$ is close to another sibling of $u$, say $w$. Without loss of generality assume that $u_{2}, u_{3}$ are close and $u_{0}, u_{1}$ are close. Observe that this case is identical to the case (i).

(iii) Either $u_{1}$ is far from $u_{2}$ or $u_{2}$ is far from $u_{3}$ and at least one child of $u$ is at distance less than $\sqrt{3}-1$. Without loss of generality assume that $u_{1}$ is far from $u_{2}$. Therefore, $d\left(u, u_{1}\right)>\sqrt{3}-1$ and $d\left(u, u_{3}\right) \leq \sqrt{3}-1$. Observe 
that $u_{3}$ is close to $u_{1}$ and $u_{2}$. By Lemma 12 either $u_{1}$ or $u_{2}$ are close to $v$ or $w$. Thus, if $v$ is close to $u_{1}$, then we can apply case (i). If $w$ is close to $u_{2}$, then let $u_{1}^{\prime}=u_{2}, u_{2}^{\prime}=u_{1}$ and $u_{3}^{\prime}=u_{3}$ and we can apply case (i) again.

(iv) $u$ has four neighbors in $T \backslash T^{\prime}$. Two cases can occur:

(i) $\left\{u_{0}, u\right\}$ is in $G^{\prime}$. Let

$$
G=\left\{G^{\prime} \backslash\left\{u_{0}, u\right\}\right\} \cup\left\{\left\{u_{1}, u\right\},\left\{u_{2}, u\right\},\left\{u_{4}, u\right\},\left\{u_{1}, u_{0}\right\},\left\{u_{2}, u_{3}\right\},\left\{u_{3}, u_{4}\right\}\right\} .
$$

If $\left(u_{0}, u\right)$ is in $\overrightarrow{G^{\prime}}$, then orient edges of $G$ as depicted in Figure 3.17a. Otherwise orient edges of $G$ as depicted in Figure 3.17b. Thus, $\vec{G}$ is strongly connected and $\Delta^{+}(\vec{G}) \leq 2$. Moreover, the leaves $u_{1}, u_{2}, u_{3}$ and $u_{4}$ of $T$ have degree one, the edges of $T$ incident to $u_{1}, u_{2}$ and $u_{4}$ are contained in $G$ and $u_{3}$ is adjacent to $u_{2}$ and $u_{4}$ in $G$. Observe that $\angle\left(u_{2} u u_{4}\right) \leq \pi / 2$.

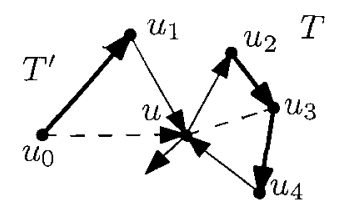

(a) $\left(u_{0}, u\right)$ is in the or1entation of $G^{\prime}$

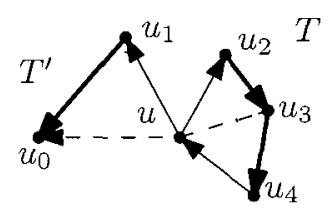

(b) $\left(u, u_{0}\right)$ is in the or1entation of $G^{\prime}$

Figure 3.17: Depicting the inductive step when $u$ has four neighbors in $T \backslash T^{\prime},\left\{u_{0}, u\right\}$ is in $G^{\prime}$. (The dashed edge $\left\{u_{0}, u\right\}$ indicates that it does not exist in $G$ but exists in $G^{\prime}$, the dotted curve is used to separate $T^{\prime}$ from $T$ and the dash dotted edge $\left\{u, u_{3}\right\}$ indicates that it exists in $T$ but not in $G$.)

(ii) $\left\{u_{0}, u\right\}$ is not in $G^{\prime}$. By inductive hypothesis $u$ is connected to ts two siblings $v$ and $w$ in $G^{\prime}$. By Lemma 12 either $u_{1}$ or $u_{4}$ is close to $v$ or $w$. Without loss of generality assume that $u_{1}$ and $v$ are close. Let $G=\left\{G^{\prime} \backslash\{v, u\}\right\} \cup$ $\left\{\left\{u_{1}, u\right\},\left\{u_{2}, u\right\},\left\{u_{4}, u\right\},\left\{v, u_{1}\right\},\left\{u_{2}, u_{3}\right\},\left\{u_{3}, u_{4}\right\}\right\}$. If $(v, u)$ is in $\vec{G}^{\prime}$, then orient edges of $G$ as depicted in Figure 3.18a. Otherwise orient edges of $G$ as depicted in Figure $3.18 \mathrm{~b}$. Thus, $\vec{G}$ is strongly connected and $\Delta^{+}(\vec{G}) \leq 2$. Moreover, $u_{1}, u_{2}, u_{3}$ and $u_{4}$ have degree one, the edges of $T$ incident to $u_{1}, u_{2}$ and $u_{4}$ are contained in $G$ and $u_{3}$ is adjacent to $u_{2}$ and $u_{4}$ in $G$.

This completes the proof of the theorem. 


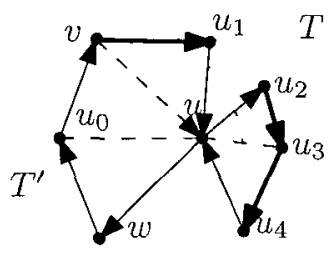

(a) $(v, u)$ is in the or1entation of $G^{\prime}$

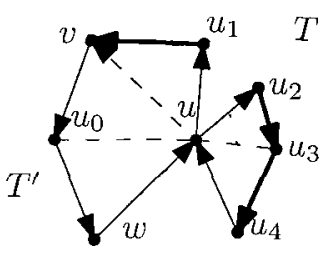

(b) $(u, v)$ is in the or1entation of $G^{\prime}$

Figure 3.18: Depicting the inductive step when $u$ has four neighbors in $T \backslash T^{\prime}$ and $\left\{u_{0}, u\right\}$ is not in $G^{\prime}$. (The dashed edge $\{v, u\}$ indicates that it does not exist in $G$ but exists in $T^{\prime}$, the dotted curve is used to separate $T^{\prime}$ from $T$, the dash dotted edge $\left\{u_{0}, u\right\}$ indicates that it exists in $T^{\prime}$ but not in $G^{\prime}$ and the dash dotted edge $\left\{u, u_{3}\right\}$ indicates that it exist in $T$ but not in $G$.)

\subsubsection{Algorithm}

In this section we present Algorthm 1 that constructs a strongly connected spanning graph with max out-degree $2 \leq k \leq 5$ and radius bounded by $2 \cdot \sin \left(\frac{\pi}{k+1}\right)$ times the optimal radius. It uses the recursive Procedure $k$ Antennae when $3 \leq k \leq 5$ and the recursive Procedure TwoAntennae when $k=2$. See the detailed algorithms for these two procedures further below.

It is not difficult to see that Algorithm 1 runs in $O(n)$ time, where $n$ is the number of vertices. The correctness of the algorithm is derived from Theorems 8,9 and 10 .

Algorithm 1: Strongly connected spanning digraph with max out-degree $2 \leq k \leq 5$ and edge length bounded by $2 \cdot \sin \left(\frac{\pi}{k+1}\right)$.

input $: T, k: T$ is an MST with max degree 5 and max length 1 , and $k$ an integer in $[2,5]$.

output: Strongly connected spanning digraph $G$ with max out-degree $k$ and range bounded by $2 \cdot \sin \left(\frac{\pi}{k+1}\right)$

1 Let $u$ be any leaf of $T$ and $v$ its neighbor in $T$;

2 Let $G \leftarrow\{(v, u),(u, v)\}$;

3 if $k=2$ then Call TwoAntennae $(G, T, v, u)$;

4 if $3 \leq k<5$ then Call $k$ Antennae $(G, T, v, u, k)$; 


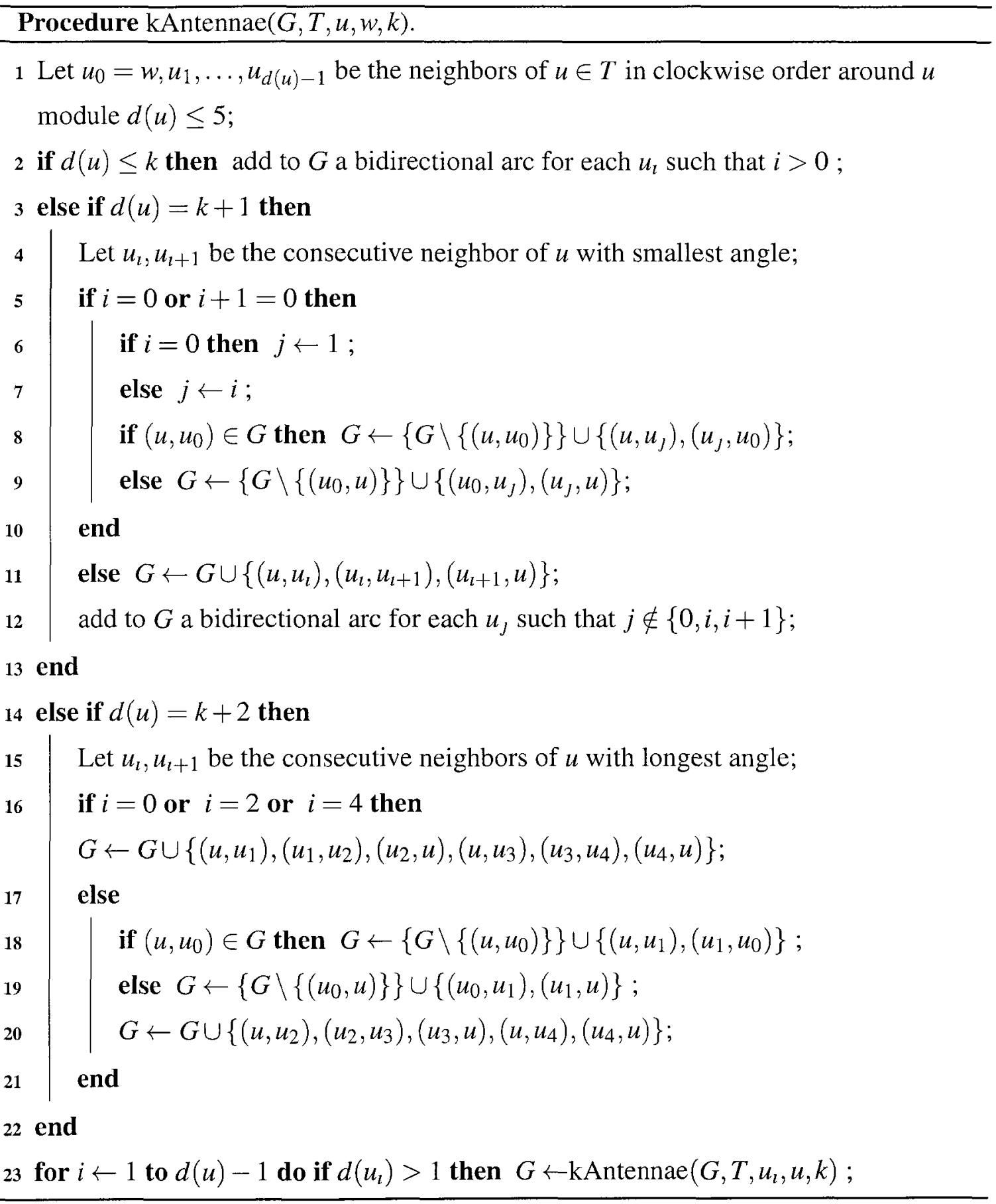

\subsection{NP hardness}

In this section we give the proof of the NP hardness result for two antennae.

Proof of Theorem 5. It is done by reduction from the well-known NP-Hard problem of 

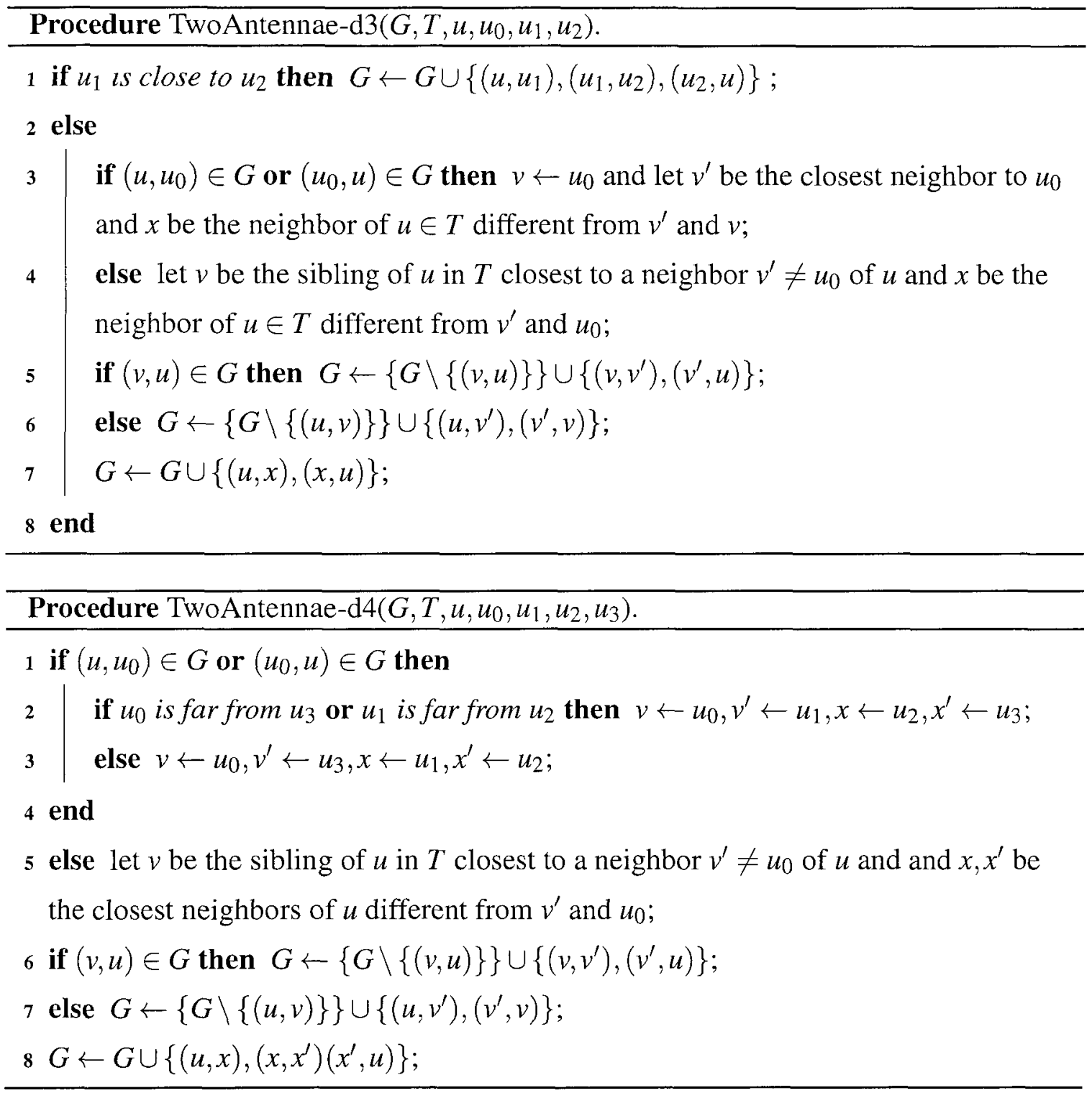

Procedure TwoAntennae-d5( $\left.G, T, u, u_{0}, u_{1}, u_{2}, u_{3}, u_{4}\right)$.

1 if $\left(u, u_{0}\right) \in G$ or $\left(u_{0}, u\right) \in G$ then $v \leftarrow u_{0}, v^{\prime} \leftarrow u_{1}, j \leftarrow 2$;

2 else let $v$ be the sibling of $u$ in $T$ closest to a neighbor $v^{\prime} \neq u_{0}$ of $u$ and $u_{J}, u_{J+1}, u_{J+2}$ be the three consecutive neighbors of $u$ different to $v^{\prime}$ and $u_{0}$;

3 if $(v, u) \in G$ then $G \leftarrow\{G \backslash\{(v, u)\}\} \cup\left\{\left(v, v^{\prime}\right),\left(v^{\prime}, u\right)\right\}$;

4 else $G \leftarrow\{G \backslash\{(u, v)\}\} \cup\left\{\left(u, v^{\prime}\right),\left(v^{\prime}, v\right)\right\}$;

$5 G \leftarrow G \cup\left\{\left(u, u_{J}\right),\left(u_{J}, u_{J+1}\right),\left(u_{J+1}, u_{J+2}\right),\left(u_{J+2}, u\right)\right\} ;$ 
Procedure TwoAntennae $(G, T, u, w)$.

1 Let $u_{0}=w, u_{1}, \ldots, u_{d(u)-1}$ be the neighbors of $u \in T$ in clockwise order around $u$;

2 if $d(u)=2$ then $G \leftarrow G \cup\left\{\left(u, u_{1}\right),\left(u_{1}, u\right)\right\}$;

3 if $d(u)=3$ then Call TwoAntennae-d $3\left(G, T, u, u_{0}, u_{1}, u_{2}\right)$;

4 if $d(u)=4$ then Call TwoAntennae-d $4\left(G, T, u, u_{0}, u_{1}, u_{2}, u_{3}\right)$;

5 if $d(u)=5$ then Call TwoAntennae-d5 $\left(G, T, u, u_{0}, u_{1}, u_{2}, u_{3}, u_{4}\right)$;

6 for $i \leftarrow 1$ to $d(u)-1$ do if $d\left(u_{l}\right)>1$ then $G=T$ woAntantennae $\left(G, T, u_{l}, u\right)$;

existence of a Hamiltonian cycle in 3-regular planar graphs. Consider a 3-regular planar graph $G=(V, E)$ and replace each vertex $v_{i}$ by a vertex-graph (meta-vertex) $G_{v_{t}}$ shown in Figure 3.19a. Furthermore, replace each edge $e=\left\langle v_{i}, v_{j}\right\rangle$ of $G$ by an edge-graph (metaedge) $G_{e}$ shown in Figure 3.19b.

Each meta-vertex has three parts connected in a cycle, with each part consisting of a pair of vertices (called connecting vertices) connected by two paths. Each meta-edge $G_{e}$ has a pair of connecting vertices at each endpoint: these vertices coincide with the connecting vertices in the corresponding parts of the meta-vertices $G_{v_{l}}$ and $G_{v_{\nu}}$. This means that after each vertex and each edge is replaced, each connecting vertex is of degree 4.

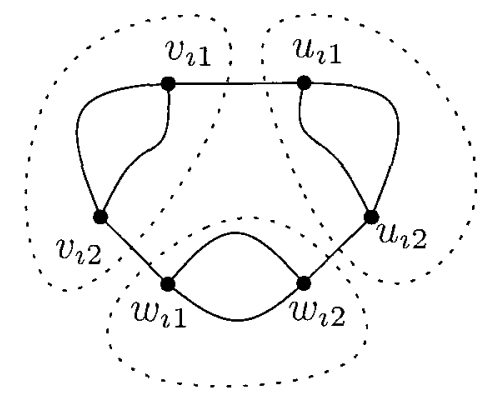

(a) Vertex graph (the dotted ovals delimit the three parts).

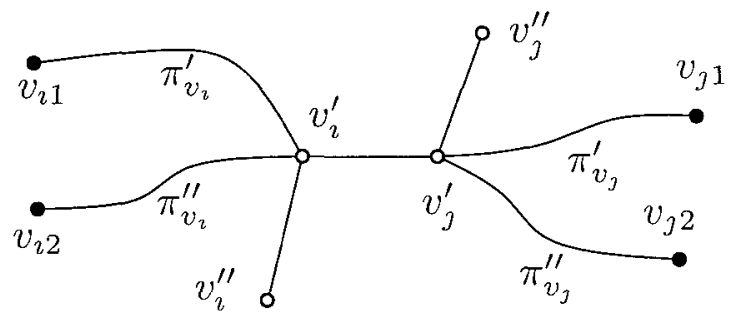

(b) Edge graph (the connecting vertices are black).

Figure 3.19: Meta-vertex and meta-edge for the NP hardness proof.

Take the resulting graph $G^{\prime}$ and embed it in the plane in such a way that:

(i) the distance (in the embedding) between neighbours in $G^{\prime}$ is at most 1 ,

(ii) the distance between non-neighbours in $G^{\prime}$ is at least $x$, and 
(iii) the smallest angle between incident edges in $G^{\prime}$ is at least $\alpha$.

Let us call the resulting embedded graph $G^{\prime \prime}$. Note that such an embedding always exists, see [9]. We have freedom to choose the length of the paths in the meta-graphs the way we need as we can stretch the configurations apart to fit everything in without violating the embedding requirements. The only constraining places are the midpoints of the metaedges and the three places in each meta-vertex where the parts are connected to each other. These can be embedded as shown in the right part of Figure 3.20. Note that the need to embed these parts without violating embedding requirements gives rise to the equations defining $x$ and $\alpha$ (see Figure 3.20). This completes the main construction.

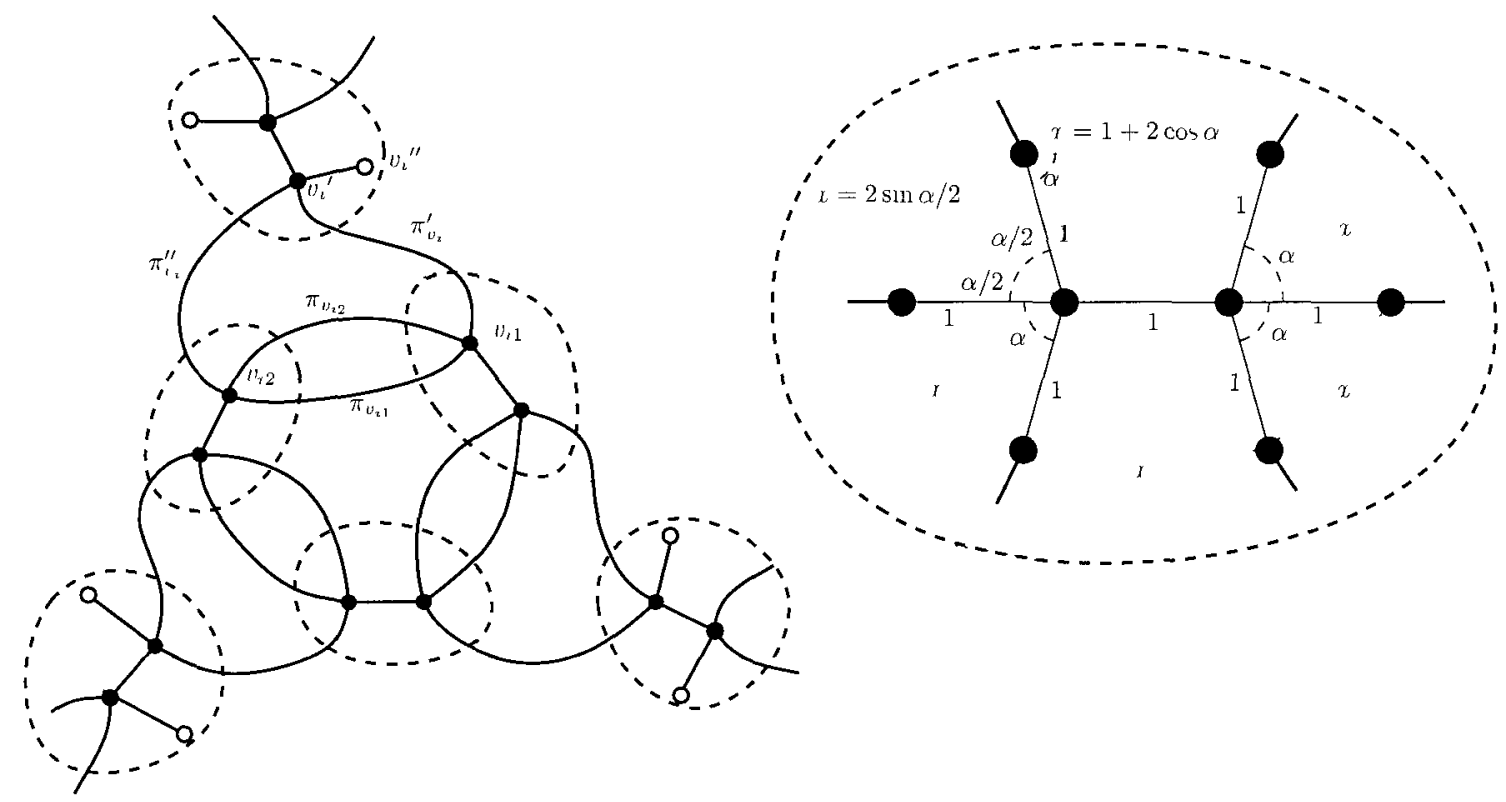

Figure 3.20: Connecting meta-edges with meta-vertices. The dashed ovals show the places where embedding is constrained.

The proof of the Theorem is based on the following claim:

Claim 1. There is a Hamiltonian cycle in $G$ if and only if there exists an assignment of two antennae with sum of angles less than $\alpha$ and range less than $x$ to the vertices of $G^{\prime \prime}$ such that the resulting connectivity graph is strongly connected.

Proof. First we show that if $G$ has a Hamiltonian cycle then there exists the assignment of such antennae that makes the resulting connectivity graph of $G^{\prime \prime}$ strongly connected. 
Figure 3.21 shows antenna assignments in the meta-edges corresponding to edges used and not used by the Hamiltonian cycle, respectively. Figure 3.22 shows the antenna assignments in a meta-vertex. Since each vertex of $G$ has one incoming, one outgoing and one unused incident edge, and each edge is either used in one direction, or not used at all, this provides the full description of antenna assignments in $G^{\prime \prime}$.

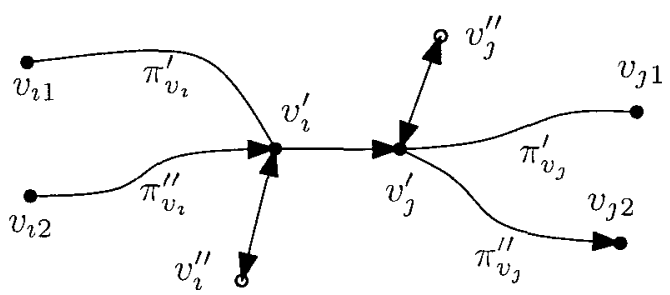

(a) An edge used in the Hamiltonian cycle from $v_{l}$ to $v_{j}$.

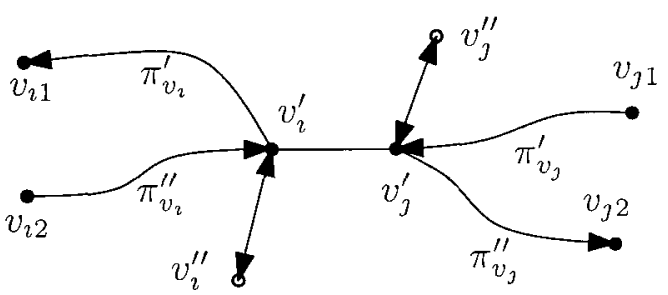

(b) An unused edge.

Figure 3.21: Antenna assignments in meta-edges.

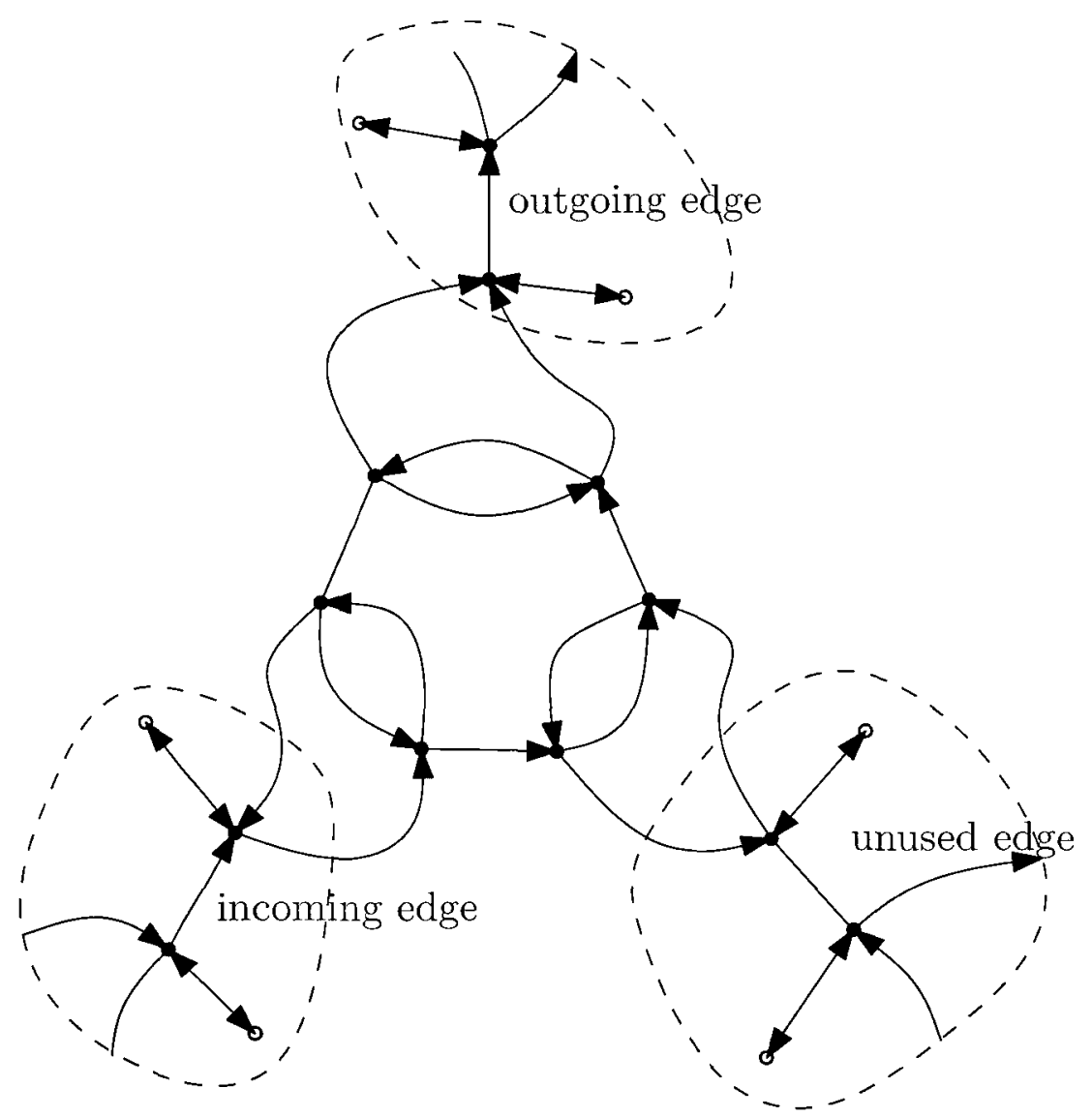

Figure 3.22: Antenna assignments at the meta-vertex and incident meta-vertices. 
Observe that the connecting pair of vertices at the meta-vertex uses two antennae towards the meta-edge it is connected to if and only if this meta-edge is outgoing; otherwise only one antenna is used towards the meta-edge and another is used towards the next part of the meta-vertex. It is easy to verify that the resulting connectivity graph is strongly connected:

(i) if the edge $e=\left\langle v_{l}, v_{J}\right\rangle$ is not used in the Hamiltonian path in the direction from $v_{l}$ to $v_{j}$, then the near half of the meta-edge $G_{e}$ (i.e. $v_{J}^{\prime}, v_{j}^{\prime \prime}, \pi_{v_{1}}^{\prime}$ and $\pi_{v_{j}}^{\prime \prime}$ ) together with the connecting part of the meta-vertex $G_{v_{j}}$ form a strongly connected subgraph,

(ii) in each meta-vertex the part corresponding to the outgoing edge is reachable from the part corresponding to the unused edge, which is in turn reachable from the part corresponding to the incoming edge, and

(iii) all vertices of a meta-edge corresponding to an outgoing edge $\left\langle v_{l}, v_{J}\right\rangle$ are reachable from either $v_{l 1}$ or $v_{l 2}$; furthermore the destination vertices $v_{j 1}$ and $v_{j 2}$ are reachable from all these vertices.

Combining these observations with the fact that the Hamiltonian cycle spans all vertices yields that the resulting graph is strongly connected.

Next we show that if it is possible to orient the antennae in $G^{\prime \prime}$ such that the resulting graph is strongly connected then there exists a Hamiltonian cycle in $G$. Recall that $G^{\prime \prime} 1 \mathrm{~s}$ constructed in such a manner that no antenna of range less than $x$ and angle less than $\alpha$ can reach two neighbouring vertices, and that no antenna can reach a vertex that is not a neighbor in $G^{\prime \prime}$.

Assume an orientation of antennae such that the resulting graph is strongly connected. First, consider a pair of connecting vertices $v_{l 1}$ and $v_{l 2}$. Since both path $\pi_{v_{11}}$ and $\pi_{v_{12}}$ are connected only to them, $v_{t 1}$ and $v_{t 2}$ must together use at least two antennae towards these two paths.

Let us call a meta-edge corresponding to edge $\left\langle v_{l}, v_{J}\right\rangle$ directed if in the connectivity graph there is an edge $\left\langle v_{l}^{\prime}, v_{j}^{\prime}\right\rangle$. Without loss of generality assume the direction is from $v_{\imath}^{\prime}$ to $v_{j}^{\prime}$, i.e. $v_{l}^{\prime}$ used an antenna to reach $v_{j}^{\prime}$. Since $v_{l}^{\prime \prime}$ is reachable only from $v_{l}^{\prime}$ (and hence $v_{l}^{\prime}$ used its second antenna on $\left.v_{l}^{\prime \prime}\right)$, this means that there is no antenna pointing from $v_{l}^{\prime}$ 
towards the paths $\pi_{v_{l}}^{\prime}$ and $\pi_{v_{i}}^{\prime \prime}$. Therefore, the only way for the vertices of these two paths to be reachable is to have both connecting vertices (which for simplicity we call $v_{l 1}$ and $v_{i 2}$, respectively) use an antenna towards these paths. Since they already used two antennae to ensure reachability of $\pi_{v_{1} 1}$ and $\pi_{v_{2} 2}$ are reachable, they have no antenna left to connect to another part of the meta-vertex.

Consider now the other half of the meta-edge. Observe that since $v_{j}^{\prime}$ must use one antenna on $v_{j}^{\prime \prime}$, it can use at most one antenna towards the paths $\pi_{v_{j}}^{\prime}$, and $\pi_{v_{j}}^{\prime \prime}$. Hence, either $v_{j 1}$ or $v_{j 2}$ must use an antenna towards one of these paths. Since these vertices must use two more antennae to ensure that the paths $\pi_{v, 1}$ and $\pi_{v, 2}$ are reachable, only one antenna is left for connecting to other parts of the meta vertex. Note that this argument holds both for receiving ends of directed meta-edges, as well as for non-directed meta-edges.

However, this means that in a meta-vertex there can be at most one outgoing directed meta-edge - otherwise there is no way to make the meta-vertex connected. Since each meta-vertex must have at least one outgoing directed meta-edge (otherwise the rest of the graph would be unreachable) and at least one incoming directed meta-edge (otherwise it would not be reachable from the rest), from the fact that the whole graph is strongly connected it follows that each meta-vertex must have exactly one undirected meta-edge, one directed incoming meta-edge and one directed outgoing meta-edge. Obviously, these correspond to unused/incoming/outgoing edges in the original graph $G$, with the directed edges forming the Hamiltonian cycle.

\subsection{Sum of Angles}

In this section we are interested in achieving strong connectivity while minimizing the sum (taken over all sensors) of angles of the antennae under the assumption that the range is set at the length of the longest incident edge. We present a trade-off between the antennae range and specified sums of antennae, given that we have $k$ directional antennae per sensor for $1 \leq k \leq 5$. The following result is proven in [7].

Lemma 13 ([7]). Assume that a node $u$ has out-degree $d$ in a graph $G$ and the sensor at $u$ is equipped with $k$ antennae, where $1 \leq k \leq d$, of range at least the maximum edge length of an edge from $u$ to its neighbors. Then $2(d-k) \pi / d$ is always sufficient and sometimes 
necessary bound on the sum of the angles of the antennae at $u$ so that there is an edge from $u$ to all its neighbors in the directed graph.

As consequence of Lemma 13 and Theorem 4 we can construct the Table 3.1 which shows trade-offs on the number, max range and sum of angles of $k$ antennae being used per sensor for the problem of converting networks of omnidirectional sensors into strongly connected network of directional sensors. Observe that for a given range, when the number of antennae increases, the angle decreases in a regular manner. The generalization of the pigeonhole principle can explain this phenomenon.

\begin{tabular}{ccl}
\hline Number of Antennae & Range (Times the Optimal Range) & Sum of Angles \\
\hline 1 & 1 & $\varphi \geq 8 \pi / 5$ \\
2 & 1 & $\varphi \geq 6 \pi / 5$ \\
3 & 1 & $\varphi \geq 4 \pi / 5$ \\
4 & 1 & $\varphi \geq 2 \pi / 5$ \\
5 & 1 & $\varphi \geq 0$ \\
1 & $2 \sin (\pi / 5)$ & $\varphi \geq 3 \pi / 2$ \\
2 & $2 \sin (\pi / 5)$ & $\varphi \geq \pi$ \\
3 & $2 \sin (\pi / 5)$ & $\varphi \geq \pi / 2$ \\
4 & $2 \sin (\pi / 5)$ & $\varphi \geq 0$ \\
1 & $\sqrt{2}$ & $\varphi \geq 4 \pi / 3$ \\
2 & $\sqrt{2}$ & $\varphi \geq 2 \pi / 3$ \\
3 & $\sqrt{2}$ & $\varphi \geq 0$ \\
1 & $\sqrt{3}$ & $\varphi \geq \pi$ \\
2 & $\sqrt{3}$ & $\varphi \geq 0$ \\
\hline
\end{tabular}

Table 3.1: Trade-off between the number, max range and sum of angles when $k$ antennae are being used by each sensor.

\subsection{Conclusion}

We have provided an algorithm which, when given as input a set of $n$ points (representing sensors) in the plane and an integer $1 \leq k \leq 5$, produces a strongly connected spanning graph so that each sensor uses at most $k$ directional antennae of angle 0 and range at most $2 \cdot \sin \left(\frac{\pi}{k+1}\right)$ times the optimal. We also show that the problem of approximating the optimal range is NP-Hard for 2 antennae when the angle is at most $9 \pi / 20$. 


\section{Chapter 4}

\section{Antenna Orientations in 3D}

\subsection{Introduction}

Although the $2 \mathrm{D}$ model that we studied in the previous chapter is simple and provides a good approximation of the reality, in a real setting it may result in a network that is not strongly connected due to the fact that the sensors may have distinct altitudes. For example, buildings, towers, mountains, cliffs, etc. To overcome this deficiency, this chapter is devoted to the study of the orientation problem in a more general setting, i.e., in the 3D space, when each sensor has one antenna. That is, given a set of points in the space with one directional antenna each sensor with solid angle $\Omega$, compute the minimum range necessary to create a strongly connected network.

\subsubsection{Preliminaries and Notation}

Given a set of points $P$ in 3D, we define the Unit Ball Graph (UBG) $G$ with parameter $r$ as the geometric graph whose vertex set is $P$ and two points are connected with a straight line edge if they are at distance at most $r$. We model a three dimensional directional antenna as a spherical sector of solid angle $\Omega$ (see Definition 14) as depicted in Figure 4.1. Hence, a directional antennae is characterized by its solid angle and range.

Definition 14. The solid angle $\Omega$ of a solid spherical sector is the ratio of the area of the spherical surface and the square of the radius of the sphere of which it forms part.

Definition 15. The apex angle $\theta$ of a spherical sector with solid angle $\Omega$ is half the maximum planar angle between any two generatrices of the spherical sector.

Using the well-known relation of Archimedes, we note that the apex angle $\theta$ and the solid angle $\Omega$ are related by the following identity.

$$
\Omega=2 \pi(1-\cos (\theta))
$$




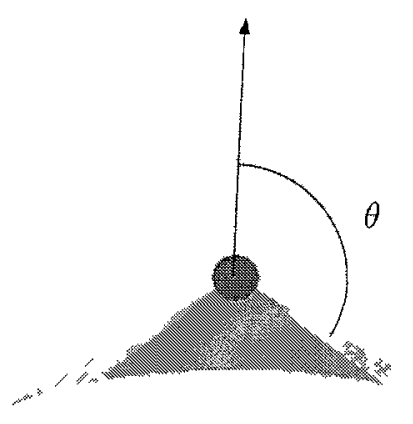

Figure 4.1: 3D directional antenna of solid angle $\Omega=2 \pi(1-\cos \theta)$.

Consider a set $S$ of points in 3D space. It is easy to see that given any solid angle the optimal range to achieve strong connectivity is at least the radius $r$ such that $\mathrm{UBG}(r)$ is connected. Thus, we can relate the optimal radius to the longest edge length $r_{M S T}(S)$ of the MST on $S$ since it is the smallest radius to achieve connectivity on $S$.

An interesting question relating to our analysis was proposed by the botanist Tammes in [66] and concerns "what is the length of the largest diameter of $n$ equal circles that can be placed on the surface of the unit sphere without overlap" (see Figure 4.2). More formally. the Tammes' radius $R_{n}$ of the unit sphere for $n$ circles is defined as the maximum radius of $n$ equal non-overlapping circles on the surface of the sphere.

The problem of finding the Tammes' radius for different values of $n$ has been studied thoroughly in the literature. In [67], Tarnai et al. have found the values of the Tammes' radius for $n \leq 12$ and $n=24$ in terms of the angle $\alpha$ it subtends at the center as shown in Figure 4.2.

\subsubsection{Our Results}

In Section 4.2, we give an algorithm that ensures optimal antenna range for the case when $\Omega \geq \frac{18 \pi}{5}$. In Section 4.3, we provide an algorithm for approximating the antennae range to $\frac{\sqrt{\Omega(4 \pi-\Omega)}}{\pi}$ the optimal range that guarantees strong connectivity of the resulting 


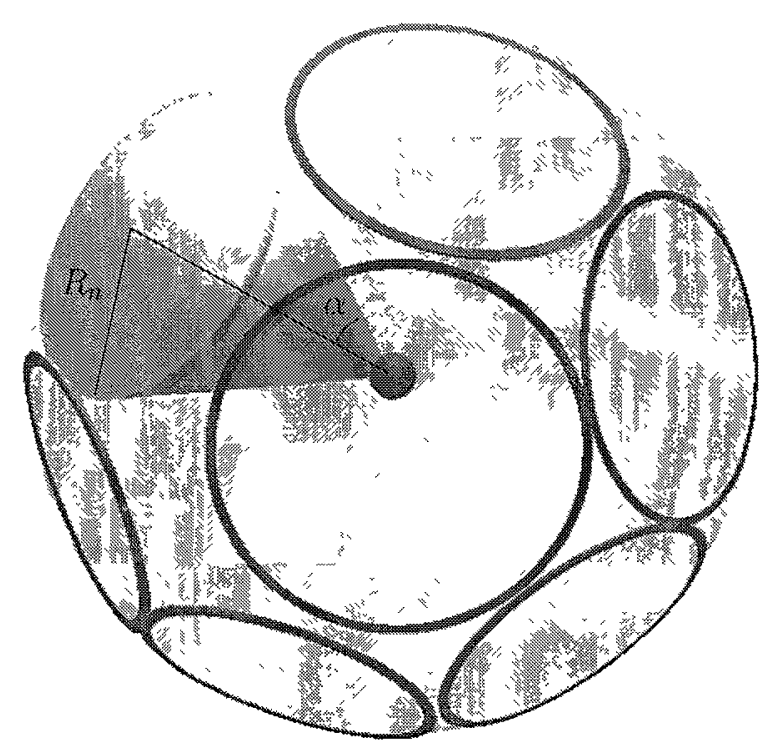

Figure 4.2: Tammes' problem.

graph when the solid angle is $2 \pi \leq \Omega<\frac{18 \pi}{5}$. In Section 4.4, we show that determining whether there exists a strong orientation of the directional antennae of solid angle $\Omega<\pi$ having optimal range is NP-Complete. In Section 4.5, we study the effect of replacing omnidirectional antennae with directional antennae on the stretch factor of the resulting network of directional antennae and present some simulation results on the variation of hop stretch factor with different network sizes and solid angles of the directional antennae.

\subsection{Lower Bound on Solid Angle and Optimal Range}

In this section, we will derive a lower bound on the solid angle of the antennae for which optimal range is sufficient for maintaining connectivity.

Theorem 16. Given a set of points $S$ in the $3 D$ space and a spherical angle $\Omega \geq \frac{18 \pi}{5}$, there exists a polynomial time algorithm having optimal range that computes a strong orientation of three dimensional antennae with solid angle $\Omega$.

Proof. Let $T$ be an MST on $S$ and $r_{M S T}(S)$ be the longest edge of $T$. Recall that the maximum degree of $T$ is bounded by 12 . For each point $p$ of $S$ we will show how to orient the antenna at $p$. Consider the sphere $B_{p}$ centered at $p$ of minimum radius $r_{p}$ that covers all the neighbors of $p$ in $T$. Observe that $r_{p} \leq r_{M S T}(S)$. For each neighbor $u$ of $p$ in $T$, 
let $u^{\prime}$ be the intersection point of $B_{p}$ with the ray emanating from $p$ toward $u$. Let $N_{B_{p}}$ be the set of points projected on the surface of $B_{p}$. Since $p$ has maximum degree 12 in $T$, $\left|N_{B_{p}}\right| \leq 12$. Let $D T_{p}$ be the Delaunay Triangulation of $N_{B_{p}}$ on the surface of $B_{p}$. Consider the largest triangle $t_{p}$ of $D T_{p}$. (In case of a tie, break it arbitrarily.) Orient the antenna at $p$ with range $r_{p}$ in such a way that $t_{p}$ is not covered. This can be done by orienting the antennae toward the opposite direction to the center of $t_{p}$.

To prove the lower bound on the solid angle at each point $p$, observe that every edge of $D T_{p}$ has length at least twice the Tammes' radius $R_{12}$ which corresponds to the kissing number in 3D [60]. Therefore, every circumcircle is at least of radius $a=2 R_{12} / \sqrt{3}$ (see Figure 4.3) and the planar angle $\alpha$ at the center of the sphere $B_{p}$ is at least $\arcsin (a)$. From [13, Problem D7, pages 114-116], it is known the exact value

$$
R_{12}=\sin \left(\frac{63^{\circ} 26^{\prime}}{2}\right) \text {. }
$$

Therefore, using Equation 4.1 we can calculate the solid angle of the antennae as follows:

$$
\begin{aligned}
\Omega & =4 \pi-2 \pi(1-\cos (\alpha)) \\
& =2 \pi(1+\cos (\alpha)) \\
& =2 \pi\left(1+\cos \left(\arcsin \left(\frac{2 R_{12}}{\sqrt{3}}\right)\right)\right) \\
& <\frac{18 \pi}{5},
\end{aligned}
$$

where the last inequality is obtained after numerical calculation. It is easy to see that the resulting transmission graph is strongly connected since every vertex covers all its neighbor in $T$. This completes the proof of the theorem.

Observe that Theorem 16 relies on the construction of the MST in 3D which takes $O\left((n \log (n))^{4 / 3}\right)$ expected time [2]. Further every other step can be done in constant time. Hence, if we do not insist on guaranteeing optimal range the algorithm can be implement in distributed manner to run in constant time assuming a location-aware setting. First we obtain the $k$-Local MST [47] of a connected UBG in constant time. Next, we orient the antennae in parallel in constant time using Theorem 16. 


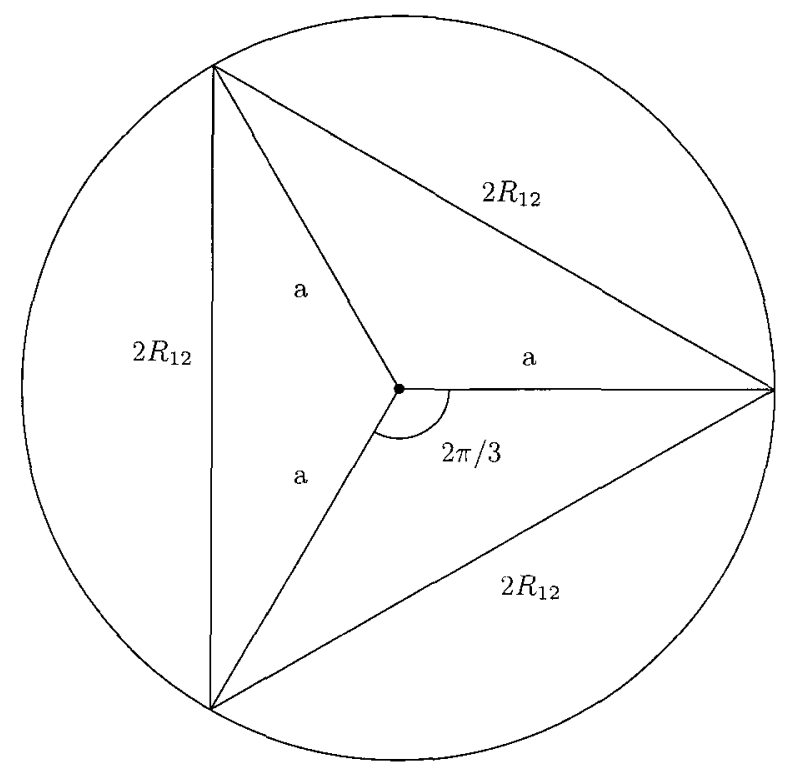

Figure 4.3: A circumcircle and the Tammes radius $R_{12}$.

\subsection{Approximation Algorithm and Upper Bound}

In this section, we propose an algorithm to create a strongly connected transmission graph when the solid angle is between $2 \pi$ and $\frac{18 \pi}{5}$.

Theorem 17. Given a set $S$ of $n$ points in the $3 D$ space and a solid angle $\Omega$ such that $2 \pi \leq \Omega<\frac{18 \pi}{5}$, it is possible to orient the antennae at each sensor with solid angle $\Omega$ and range at most $r(\Omega)$ times the optimal range in $O\left((n \log (n))^{4 / 3}\right)$ expected time so that the transmission graph is connected, where

$$
r(\Omega)=\frac{\sqrt{\Omega(4 \pi-\Omega)}}{\pi} \cdot r_{M S T}(S)
$$

Proof. Let $T$ be an MST on $S$ and $r_{M S T}(S)$ be the longest edge of $T$. Consider a maximum matching $M$ of $T$ with the property that every internal vertex of $T$ is incident to an edge in $M$. It is not difficult to see that $M$ always exists with this property. To see this, consider an internal vertex $v$ that is not incident to an edge in $M$ and let $u$ be a leaf of $T$ incident to an edge in $M$. Observe that $u$ always exists since $M$ is maximum. Thus, we can obtain a set $M^{\prime}$ that includes $v$ by considering an alternating path from $v$ to $u$.

We say that the endpoints of an edge in $M$ form a couple. We use sectors of solid angle $\Omega$ and radius $r(\Omega)$ at each point and orient them as follows: At each node $u \in S$ not 
incident to an edge of $M$, the sector is oriented so that it induces the directed edge from $u$ to its parent in $T$ in the corresponding transmission graph. For each pair of points $u$ and $v$ forming a couple, we orient the sector at $u$ so that it contains all points at distance $r(\Omega)$ from $u$ for which the counter-clockwise angle $\angle v u p$ is in $[0,2 \theta]$ where $2 \theta$ is the apex angle of the directional antenna; see Figure 4.4.

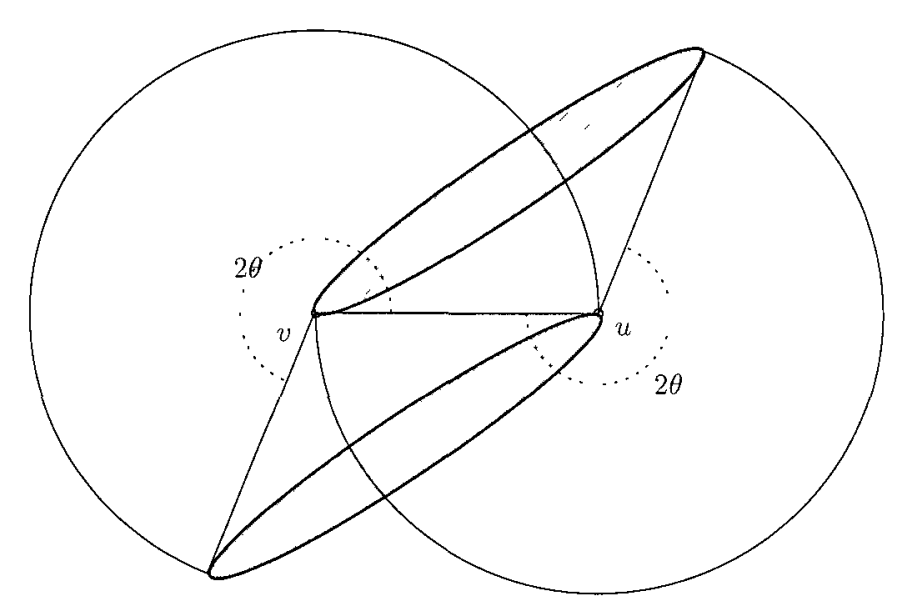

Figure 4.4: Orientation of antennae at $u$ and $v$ such that $(u, v)$ is in the matching $M$.

Now, we will prove that the resulting transmission graph is connected. To prove this, we first prove that the transmission graph has the following property $P$ :

For each pair of points $u$ and $v$ forming a couple, the transmission graph $G$ contains two opposite directed edges between $u$ and $v$ and for each neighbor $w$ of either $u$ or $v$ in $T$, it contains a directed edge from either $u$ or $v$ to $w$.

Consider a point $w$ corresponding to a neighbor of $u$ in $T$ (the argument for the case where $w$ is a neighbor of $v$ is symmetric). Let $\theta$ be the apex angle of $\Omega$. Clearly, $w$ is at distance $d(u, w) \leq r_{M S T}(S)$ from $u$. Since $\Omega \leq \frac{18 \pi}{5}$, using Equation 4.2, we have the antenna range, $r(\Omega) \geq 6 r_{M S T}(S) / 5$. Hence, $w$ is contained in the spherical sector of $u$ if the counter-clockwise planar angle $\angle v u w$ is at most $\theta$. Now, assume that the angle $\angle v u w>\theta$. By the law of cosines in the triangle defined by points $u, v$ and $w$, we have that $d(v, w)$ is equal to

$$
\sqrt{d(v, w)^{2}+d(u, v)^{2}-2 d(u, v) d(u, w) \cos (\angle v u w)}
$$


It follows that

$$
\begin{aligned}
d(v, w) & \leq \sqrt{2-2 \cos (2 \theta)} \cdot r_{M S T}(S) \\
& \leq|2 \sin (\theta)| \cdot r_{M S T}(S) \\
& \leq\left|2 \sqrt{1-\cos ^{2}(\theta)}\right| \cdot r_{M S T}(S) \\
& \leq\left|2 \sqrt{1-\left(1-\frac{\Omega}{2 \pi}\right)^{2}}\right| \cdot r_{M S T}(S) \\
& \leq \frac{\sqrt{\Omega(4 \pi-\Omega)}}{\pi} \cdot r_{M S T}(S),
\end{aligned}
$$

since the apex angle satisfies $2 \theta>\pi$, for all $\Omega \geq 2 \pi$. Hence, in the above argument, either $\angle v u w$ or $\angle u v w$ is less than $\pi$ which means the transmission graph contains an edge from either $u$ or $v$ to $w$. This completes the proof of property $P$.

It is not difficult to see that the resulting transmission graph is strongly connected since each leaf not incident to an edge in $M$ is connected to its parent and property $\mathcal{P}$ guarantees a directed path between the vertices of any couple and their neighbors.

Regarding the complexity, the construction of the MST can be done in $O\left((n \log (n))^{4 / 3}\right)$ expected time. It is not difficult to see that all the rest of the steps can be implemented in linear time. This completes the proof of Theorem 17.

\subsection{The Complexity of the 3D Case}

In this section, we will prove that when the solid angle of the directional antennae is less than $\pi$, the problem of finding out an orientation of the sensors such that the transmission graph is connected is NP-Complete.

Theorem 18. Given a set of points $S$ in the $3 D$ space and $\Omega<\pi$ determining whether there exists a strong orientation of directional sensors of solid angle $\Omega$ having optimal range is NP-Complete.

Proof. It is easy to see that the problem is in NP. We prove the NP-Hardness by using a result known for the $2 \mathrm{D}$ case. In [10], the authors have proved that in case of directional antennae in $2 \mathrm{D}$ modelled as a circular sector, when the sector angle is less than $\pi$, the problem of maintaining connectivity is NP-Complete. 
Consider a set $S$ of points in the plane. We will prove that the 2D problem is equivalent to the 3D problem. From Definition 15 and Archimedes' relation, any plane that cuts the coverage area of any $3 \mathrm{D}$ directional antennae through the apex with angle $\Omega$ has plane angle satisfying $\cos (\theta) \leq 1-\frac{\Omega}{2 \pi}$. Therefore, $\theta \leq 2 \pi / 3$ if and only if $\Omega \leq \pi$. Assume a strong orientation of the plane directional antenna of $S$ with angle at most $2 \pi / 3$. Clearly, we can orient the 3D directional antennae with solid angle $\pi$ in such a way that they cover the plane angle. Similarly, if there exists an algorithm that creates a strong orientation of the 3D directional antenna of $S$ with solid angle $\pi$ then we can orient the 2D directional antenna of $S$ with angle $2 \pi / 3$ to form a strongly connected graph. This completes the proof of the theorem.

\subsection{Simulation Results}

In this section, we study the impact in the hop stretch factor of replacing 3D omnidirectional antennae with 3D directional antennae. In our experiments we randomly generate sets of $n$ points in the 3D space. For each instance $S$ we construct the directional network, say $G$, with antenna beam-width $\Omega$ according to Theorem 17 as well as the unit ball graph UBG $\left(r_{M S T}(S)\right)$. Then, we compare the average shortest path of $G$ with the average shortest path of $\mathrm{UBG}\left(r_{M S T}(S)\right)$.

In the first simulation we fix $\Omega$ to $13 \pi / 5$ and vary the number of vertices from 30 to 100 in increments of 10 . We ran 30 times each value of $n$ and plot the ratio with the boxdiagram as depicted in Figure 4.5. Observe that in all the cases the third quantile of the ratio is less than one and just a few values are greater than one. Thus, in general we can conclude that with $\Omega=13 \pi / 5$ the directed network behaves better than the $\operatorname{UBG}\left(r_{M S T}(S)\right)$. A possible explanation is because the radius of the 3D directional antennae is greater than $r_{M S T}(S)$ and $U B G\left(r_{M S T}(S)\right)$ has optimal range.

In the second simulation we fix $n$ to 70 and consider distinct values of the solid angle

$\Omega$ in the range $\left(2 \pi, \frac{18 \pi}{5}\right)$. We ran 30 times each value of $\Omega$ and plot the ratio with the box-diagram as depicted in Figure 4.6. Similarly, in all the cases the third quantile of the ratio is less than one. However, it seems that when $\Omega$ grows, the ratio also increases. Thus, we can conclude that when $\Omega$ is small, the directed network behaves better than 


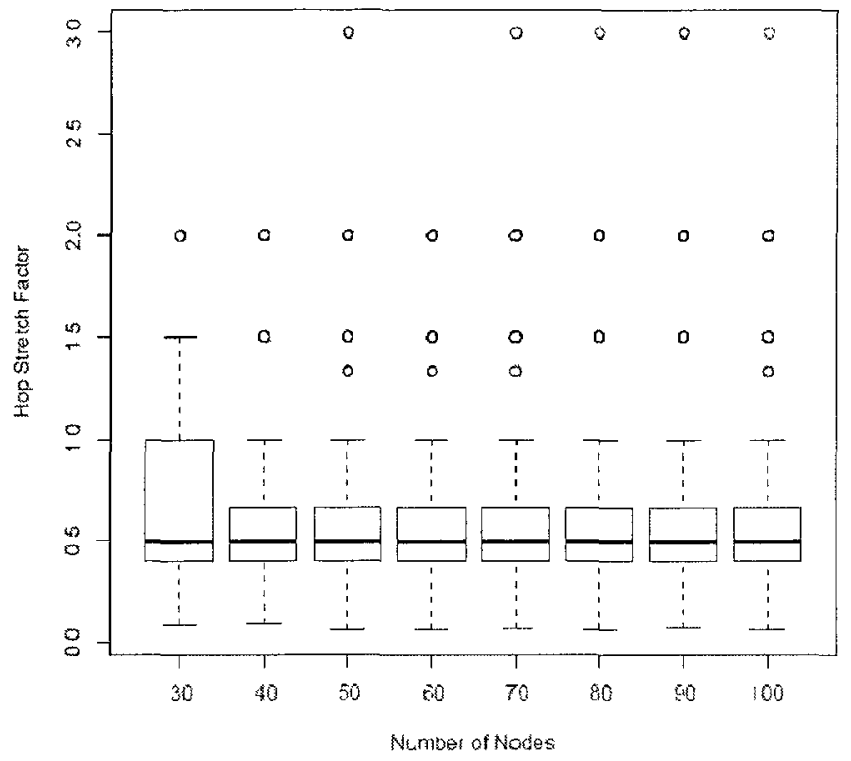

Figure 4.5: Directed Transmission Graph/Unit Ball Graph varying $n$. (Hop stretch factor of the average shortest paths.)

the $\operatorname{UBG}\left(r_{M S T}(S)\right)$. A possible reason for the phenomenon is that when $\Omega$ increases, $r(\Omega)$ converges to the optimal value.

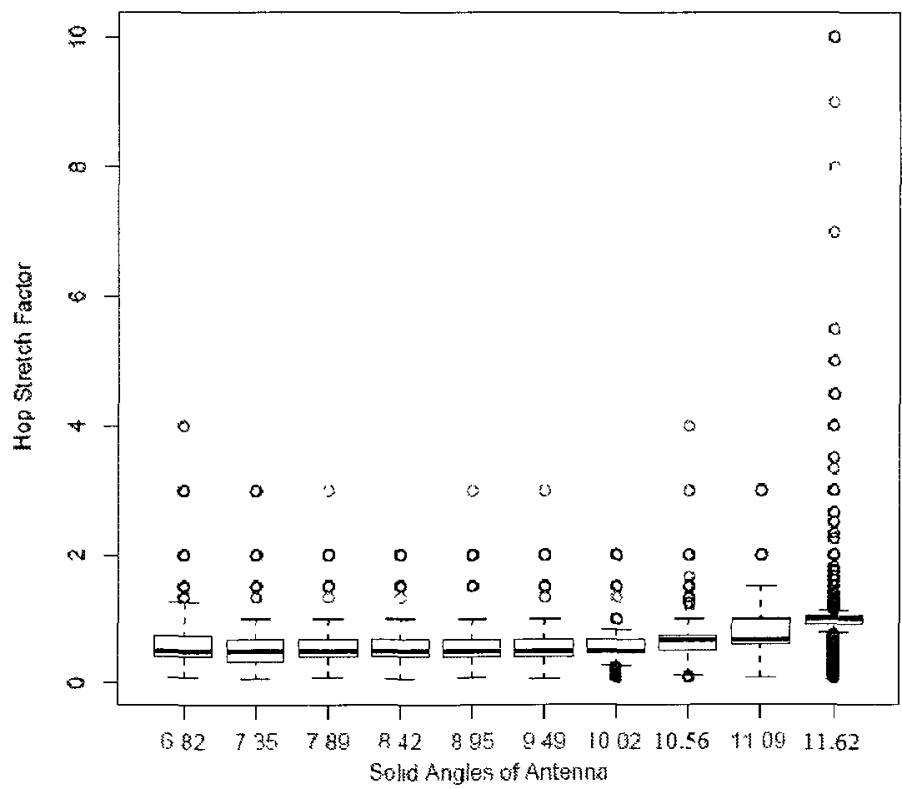

Figure 4.6: Directed Transmission Graph/Unit Ball Graph varying $\Omega$. (Hop stretch factor of the average shortest paths.) 


\subsection{Conclusion}

In this chapter we considered the antenna orientation problem in 3D. 3D directional antennae were modelled as spherical sectors of solid angle $\Omega$. Using this model, we gave a trade-off between the angle and the transmission range when each sensor has one antenna. 


\section{Chapter 5}

\section{Orientation of 2-Edge Connected Planar Graph}

\subsection{Introduction}

In this chapter, we study the problem of directing edges of an undirected (connected) planar graph in such a way that the resulting digraph spans all vertices, is strongly connected, has bounded hop stretch factor, and the number of arcs employed is minimized. Note that if the undirected graph is Hamiltonian then a solution is to orient edges along a Hamilton cycle. This yields an orientation that is strongly connected and has the minimum possible number of arcs. However the hop stretch factor of such orientation is unbounded. On the other hand, one can orient every edge of an undirected graph in two opposite directions. This will result in an orientation that is strongly connected with hop stretch factor equal to one. However the number of arcs in such an orientation is largest possible. Hence, we are looking for trade-offs between these two approaches.

\subsubsection{Preliminaries and Notation}

Given two integers $a<b,[a, b]=\{a, a+1, \ldots, b\}$ denote the integer interval. A (face) $\lambda$-coloring $\Lambda: F \rightarrow[1, \lambda]$ of a planar graph $G(V, E, F)$ is an assignment $\Lambda$ of $\lambda$ colors to faces of $G$ such that adjacent faces, i.e. faces sharing a common edge, are assigned distinct colors.

Recall than an orientation $\vec{G}$ of a graph $G$ is a digraph obtained from $G$ by orienting every edge of $G$ in at least one direction. Let $d^{+}(u)$ denote the out-degree of $u$ in $\vec{G}$. Similarly, by $d(f)$ we denote the degree (the number of edges) of the face $f$ in a planar graph (digraph) $G$. In both cases, if an ambiguity can occur, we expand the notation by a subscript representing the corresponding graph, e.g. $d_{\vec{G}}^{+}(u)$ denotes the out-degree of $u$ in the digraph $\vec{G}$. Finally let $\Phi(G)$ be the maximum degree of a face in $G$, i.e. $\Phi(G)=\max _{f \in F} d(f)$. 


\subsubsection{Our Results}

Let $G=(V, E)$ be a connected planar graph without cut edges and let $\Lambda$ be a face coloring, say with $\lambda$ colors. We present three polynomial constructions for orientations of $G$.

The first construction (presented in Section 5.2) gives a strong orientation with at most $\left(2-\frac{4 \lambda-6}{\lambda(\lambda-1)}\right)|E|$ arcs and the hop stretch factor at most $\Phi(G)-1$. The second construction (presented in Section 5.3) gives a strong orientation with at most $|E|$ arcs and the hop stretch factor at most $(\Phi(G)-1)^{\left\lceil\frac{\lambda+1}{2}\right\rceil}$. The third construction (presented in Section 5.4) can be applied to planar graphs which are 3-edge connected. It uses a particular 6 -face coloring and for any integer $k \geq 1$ produces a strong orientation with at most $\left(1-\frac{k}{10(k+1)}\right)|E|$ arcs and the hop stretch factor at most $\Phi^{2}(G)(\Phi(G)-1)^{2 k+4}$.

\subsection{Orientations with more than $|E|$ Arcs}

Let $G(V, E, F)$ be a simple planar geometric graph. We want to orient edges in $E$ so that the resulting digraph is strongly connected. A trivial algorithm is to orient each edge in $E$ in both directions. In this case, the number of arcs is $2|E|$ and the hop stretch factor is 1. In this section we prove that it is possible to orient less than $2|E|$ edges of $G$ and still maintain bounded hop stretch factor. Our approach is based on a $\lambda$-coloring of faces in $F$, for some integer $\lambda$. The idea of employing face coloring was used in [76] to triangulate 2-connected geometric graphs. Intuitively we give directions to edges depending on the color of their incident faces.

Theorem 19. Let $G(V, E, F)$ be a planar geometric graph which is 2-edge connected. Suppose $G$ has a face $\lambda$-coloring for some integer $\lambda$. There exists a strongly connected orientation $\vec{G}$ with at most

$$
\left(2-\frac{4 \lambda-6}{\lambda(\lambda-1)}\right) \cdot|E|
$$

arcs, so that its hop stretch factor is at most $\Phi(G)-1$.

Before giving the proof, we introduce some useful ideas and preliminary results that will be required.

Consider a planar geometric graph $G(V, E, F)$ and a face $\lambda$-coloring $\Lambda$ of $G$ with colors $\{1,2, \ldots, \lambda\}$. Let $\vec{G}$ be the orientation resulting from giving two opposite directions to each 
edge in $E$. For each $\operatorname{arc}(u, v)$, we define $L_{u, v}$ as the face which is incident to $\{u, v\}$ on the left of $(u, v)$, and similarly $R_{u, v}$ as the face which is incident to $\{u, v\}$ on the right of $(u, v)$. Observe that for given embedding of $G, L_{u, v}$ and $R_{u, v}$ are well defined. Since $G$ has no cut edges, $L_{u, v} \neq R_{u, v}$. This will be always assumed in the proofs below without specifically recalling the reason again. We classify arcs according to the colors of their incident faces. Let $E_{l, J}$ be the set of $\operatorname{arcs}(u, v)$ in $\vec{G}$ such that $\Lambda\left(L_{u, v}\right)=i$ and $\Lambda\left(R_{u, v}\right)=j$. It is easy to see that each arc is exactly in one such set. Hence, the following lemma is evident and can be given without proof.

Lemma 20. For any face $\lambda$-coloring of a planar geometric graph $G$,

$$
\sum_{i=1}^{\lambda} \sum_{j=1, j \neq l}^{\lambda}\left|E_{l, j}\right|=2|E|
$$

For any of $\lambda(\lambda-1)$ ordered pairs of two distinct colors $a$ and $b$ in the coloring $\Lambda$, we define the digraph $D(G ; a, b)$ as follows: The vertex set of the digraph $D$ is $V$ and the arc set of $D$ is

$$
\bigcup_{t \in[1, \lambda] \backslash \backslash\{b\}, j \in[1, \lambda] \backslash\{a\}} E_{l, J} .
$$

Along with this definition, for $i \neq b, j \neq a$, and $i \neq j$, we say that $E_{l, j}$ is in $D(G ; a, b)$. Next consider the following characteristic function

$$
\chi_{a, b}\left(E_{l, J}\right)= \begin{cases}1 & \text { if } E_{l, J} \text { is in } D(G ; a, b), \text { and } \\ 0 & \text { otherwise. }\end{cases}
$$

We claim that every set $E_{l, J}$ is in exactly $\lambda^{2}-3 \lambda+3$ different digraphs $D(G ; a, b)$ for some $a \neq b$.

Lemma 21. For any face $\lambda$-coloring of a planar geometric graph $G$,

$$
\sum_{a=1}^{\lambda} \sum_{b=1, b \neq a}^{\lambda} \chi_{a, b}\left(E_{l, J}\right)=\lambda^{2}-3 \lambda+3
$$

Proof. Let $i, j \in[1, \lambda], i \neq j$ be fixed. For any two distinct colors $a$ and $b$ of the $\lambda$-coloring of $G, \chi_{a, b}\left(E_{l, j}\right)=1$ only if either $i=a$, or $j=b$, or $i$ and $j$ are different from $a$ and $b$. There are $(\lambda-1)+(\lambda-2)+(\lambda-2)(\lambda-3)$ such colorings. The lemma follows by simple counting. 
The following lemma gives a key property of the digraph $D(G ; a, b)$.

Lemma 22. Given a face $\lambda$-coloring of a planar geometric graph $G$ with no cut edges, and the corresponding digraph $D(G ; a, b)$. Every face of $D(G ; a, b)$, which has color $a$, constitutes a counter clockwise directed cycle, and every face which has color b, consttututes a clockwise directed cycle. All arcs on such cycles are unidirectional. Moreover, each arc of $D(G ; a, b)$ incident to faces having colors different from either $a$ or $b$ is bidirectional.

Proof. Let $G$ be a planar geometric graph with a face $\lambda$-coloring $\Lambda$ with colors $a, b$ and $\lambda-2$ other colors. Consider $D(G ; a, b)$. The sets $E_{a, x}$ are in $D(G ; a, b)$ for each color $x \neq a$. Let $f$ be a face and let $\{u, v\}$ be an edge of $f$ so that $L_{u, v}=f$. Let $f^{\prime}$ be the other face incident to $\{u, v\}$; hence $R_{u, v}=f^{\prime}$.

Since $G$ has no cut edges, $f \neq f^{\prime}$, and since $\Lambda\left(f^{\prime}\right) \neq a$, the $\operatorname{arc}(u, v) \in \bigcup_{x \neq a} E_{a, \lambda}$ and hence the $\operatorname{arc}(u, v)$ is in $D(G ; a, b)$. Since $\{u, v\}$ was an arbitrary edge of $f, f$ will induce a counter clockwise cycle in $D(G ; a, b)$. The fact that every face which has color $b$ induces a clockwise cycle in $D(G ; a, b)$ is similar.

Finally consider an edge $\{u, v\}$ such that $\Lambda\left(L_{u, v}\right) \neq a, b$ and $\Lambda\left(R_{u, v}\right) \neq a, b$. Hence $(u, v) \in E_{\lambda, d}$ which is in $D(G ; a, b)$ and similarly $(v, u) \in E_{d, \lambda}$ which is also in $D(G ; a, b)$. This proves the lemma.

We are ready to prove Theorem 19 .

Theorem 19. Let $G$ be a planar geometric graph having no cut edges. Let $\Lambda$ be a face $\lambda$-coloring of $G$ with colors $a, b$, and other $\lambda-2$ colors. Suppose colors $a$ and $b$ are such that the corresponding digraph $D(G ; a, b)$ has the minimum number of arcs. Consider $\bar{A}$ the average number of arcs in all digraphs arising from $\Lambda$. Thus,

$$
\begin{gathered}
\bar{A}=\frac{1}{\lambda(\lambda-1)} \sum_{a=1}^{\lambda} \sum_{b=1, b \neq a}^{\lambda}|D(G ; a, b)|, \text { where } \\
|D(G ; a, b)|=\sum_{l=1}^{\lambda} \sum_{\jmath=1, j \neq l}^{\lambda} \chi_{a, b}\left(E_{l, j}\right)\left|E_{l, j}\right| .
\end{gathered}
$$


By Lemma 20 and Lemma 21,

$$
\begin{aligned}
\bar{A} & =\frac{1}{\lambda(\lambda-1)} \sum_{a=1}^{\lambda} \sum_{b=1, b \neq a}^{\lambda} \sum_{i=1}^{\lambda} \sum_{J=1, j \neq i}^{\lambda} \chi_{a, b}\left(E_{i, j}\right)\left|E_{l, j}\right| \\
& =\frac{1}{\lambda(\lambda-1)} \sum_{i=1}^{\lambda} \sum_{j=1, j \neq i}^{\lambda}\left(\lambda^{2}-3 \lambda+3\right)\left|E_{l, j}\right| \\
& =\frac{2\left(\lambda^{2}-3 \lambda+3\right)}{\lambda(\lambda-1)}|E| \\
& =\left(2-\frac{4 \lambda-6}{\lambda(\lambda-1)}\right)|E| .
\end{aligned}
$$

Hence $D(G ; a, b)$ has at most the desired number of arcs.

To prove the strong connectivity of $D(G ; a, b)$, consider any path, say $u=u_{0}, u_{1}, \ldots, u_{n}=$ $v$, in the graph $G$ from $u$ to $v$. We prove that there exists a directed path from $u$ to $v$ in $D(G ; a, b)$. It is enough to prove that for all $i$ there is always a directed path from $u_{i}$ to $u_{i+1}$ for any edge $\left\{u_{l}, u_{i+1}\right\}$ of the above path. We distinguish several cases.

- Case 1. $\Lambda\left(L_{u_{l}, u_{i+1}}\right)=a$. Then $\left(u_{i}, u_{i+1}\right) \in E_{a, \omega}$ where $\omega=\Lambda\left(R_{u_{i}, u_{i+1}}\right)$. Since $E_{a, \omega}$ is in $D(G ; a, b)$, the arc $\left(u_{l}, u_{i+1}\right)$ is in $D(G ; a, b)$. Moreover, the hop stretch factor of $\left\{u_{i}, u_{i+1}\right\}$ is one.

- Case 2. $\Lambda\left(L_{u_{r}, u_{l+1}}\right)=b$. Hence, $\left(u_{i}, u_{i+1}\right)$ is not in $D(G ; a, b)$. However, by Lemma 22, the face $L_{u_{t}, u_{t+1}}=R_{u_{t+1}, u_{t}}$ constitutes a clockwise directed cycle, and therefore, a directed path from $u_{i}$ to $u_{i+1}$. It is easy to see that the hop stretch factor of $\left\{u_{i}, u_{l+1}\right\}$ is not more than the size of the face $L_{u_{1}, u_{l+1}}$ minus one, which is at most $\Phi(G)-1$.

- Case 3. $\Lambda\left(L_{u_{t}, u_{t+1}}\right) \neq a, b$. Suppose $\Lambda\left(L_{u_{t}, u_{t+1}}\right)=c$. Three cases can occur.

$-\Lambda\left(R_{u_{i}, u_{i+1}}\right)=a$. Hence, $\left(u_{i}, u_{i+1}\right)$ is not in $D(G ; a, b)$. However, by Lemma 22 , there exists a counter clockwise directed cycle around face $R_{u_{t}, u_{t+1}}=L_{u_{l+1}, u_{i}}$, and consequently a directed path from $u_{i}$ to $u_{i+1}$. The hop stretch factor is at most the size of face $R_{u_{1}, u_{1+1}}$ minus one, which is at most $\Phi(G)-1$.

$-\Lambda\left(R_{u_{t}, u_{1+1}}\right)=b$. By Lemma 22, there exists a clockwise directed cycle around face $R_{u_{l}, u_{l+1}}$. This cycle contains $\left(u_{i}, u_{i+1}\right)$, and in addition the hop stretch factor of $\left\{u_{i}, u_{i+1}\right\}$ is one. 
- $\Lambda\left(R_{u_{t} u_{t+1}}\right)=d \neq a, b, c$. By the construction, $D(G ; a, b)$ has both $\operatorname{arcs}\left(u_{l}, u_{t+1}\right)$ and $\left(u_{t+1}, u_{l}\right)$. Again, the hop stretch factor of $\left\{u_{t}, u_{t+1}\right\}$ is one.

This proves the theorem.

As indicated in Theorem 19 the number of arcs in the orientation depends on the number $\lambda$ of colors. Thus, for specific values of $\lambda$ we have the following table of values:

\begin{tabular}{|c|c|c|c|c|c|}
\hline$\lambda$ & 3 & 4 & 5 & 6 & 7 \\
\hline $2-\frac{4 \lambda-6}{\lambda(\lambda-1)}$ & 1 & $\frac{7}{6}$ & $\frac{13}{10}$ & $\frac{7}{5}$ & $\frac{31}{21}$ \\
\hline
\end{tabular}

Regarding the complexity of the algorthm, this depends on the number $\lambda$ of colors being used. For example, computing a 4-coloring can be done in $O\left(n^{2}\right)$ [59]. Finding the digraph with minimum number of arcs among the twelve possible digraphs can be done in linear time. Therefore, for $\lambda=4$, the orientation can be computed in $O\left(n^{2}\right)$. For $\lambda=5$ a 5 coloring can be found in $O(n)$ time. For geometric planar subs-graphs of unit disk graphs and location aware nodes there is a local 7-coloring (see [15]). For more information on colorings the reader is advised to look at [31]. We also have the following corollary.

Corollary 23. Let $G=(V, E, F)$ be a geometric planar triangulation. There exists a strongly connected ortentation $\vec{G}$ with at most $\frac{7(|V|-1)}{2}-\frac{7 k}{6}$ arcs and hop stretch factor less than $k$; where $k$ is the degree of the outer face.

\subsection{Orientations with $|E|$ Arcs}

Theorem 19 shows that every geometric planar graph $G$ without cut edges has a strong orientation with bounded hop stretch factor and at most $\left(2-\frac{4 \lambda-6}{\lambda(\lambda-1)}\right) \cdot|E|$ arcs. In this section we show that one can orient every edge in exactly one direction only and still obtain a strong orientation. However the hop stretch factor will increase.

Consider a geometric planar graph $G(V, E, F)$ having no cut edges and a face $\lambda$ coloring $\Lambda$ of $G$ with colors $[1, \lambda]$. Let $\vec{G}$ be the orientation assigning two opposite directions to each edge of $E$. Let $E_{l, \jmath}$ be the set of $\operatorname{arcs}(u, v)$ in $\vec{G}$ such that $\Lambda\left(L_{u, v}\right)=\imath$ and $\Lambda\left(R_{u, v}\right)=j$. Recall that since $G$ has no cut edges, $L_{u, v} \neq R_{u, v}$. Clearly, these sets are pairwise disjoint. We define the digraph $D(G ; \Lambda)$ as follows: The vertex set of the digraph $D(G ; \Lambda)$ is $V$ and the arc set of $D(G ; \Lambda)$ is $\bigcup_{l<J \leq \lambda} E_{l, j}$. 
It is not difficult to observe that in $D(G ; \Lambda)$ exactly one direction is assigned to every edge of $G$.

Theorem 24. Let $G(V, E, F)$ be a geometric planar graph which is 2-edge connected. For any face $\lambda$-coloring $\Lambda$ of $G$, the digraph $D(G ; \Lambda)$ is strongly connected, has exactly $|E|$ arcs, and its hop stretch factor is at most $(\Phi(G)-1)^{\left\lceil\frac{\lambda+1}{2}\right\rceil}$.

Proof. We already observed above that $D(G ; \Lambda)$ has $|E|$ arcs. We prove the following two statements.

(i) We first prove by induction on $k$ that if $\{u, v\} \in E$ so that $\Lambda\left(L_{, v}\right)=k$ then if $(u, v)$ is in $D(G ; \Lambda)$ then there is also a directed path from $v$ to $u$ in $D(G ; \Lambda)$ of length at most $(\Phi(G)-1)^{k}$ such that every arc on this path is incident to a face of color at most $k$.

(ii) Second we prove that for every $k$ if $\{u, v\} \in E$ so that $\Lambda\left(R_{u, v}\right)=k$ then if $(v, u)$ is in $D(G ; \Lambda)$ then there is also a directed path from $u$ to $v$ in $D(G ; \Lambda)$ of length at most $(\Phi(G)-1)^{\lambda-k+1}$ such that every arc on this path is incident to a face of color at least $k$.

The theorem follows easily. Indeed, let $\{u, v\} \in E$ so that $\Lambda\left(L_{u, v}\right)<\Lambda\left(R_{u, v}\right)$. The arc $(u, v)$ constitutes the required directed path from $u$ to $v$. We exhibit required directed path from $v$ to $u$ as follows. If $\Lambda\left(L_{u, v}\right) \leq\left\lceil\frac{\lambda}{2}\right\rceil$, then the directed path from $v$ to $u$ exists by first statement. If $\Lambda\left(L_{u, v}\right)>\left\lceil\frac{\lambda}{2}\right\rceil$, then since $L_{u, v}=R_{v, u}, \Lambda\left(R_{v, u}\right)>\left\lceil\frac{\lambda}{2}\right\rceil$ and since $(u, v) \in E$, the required directed path from $v$ to $u$ exists by the second statement above.

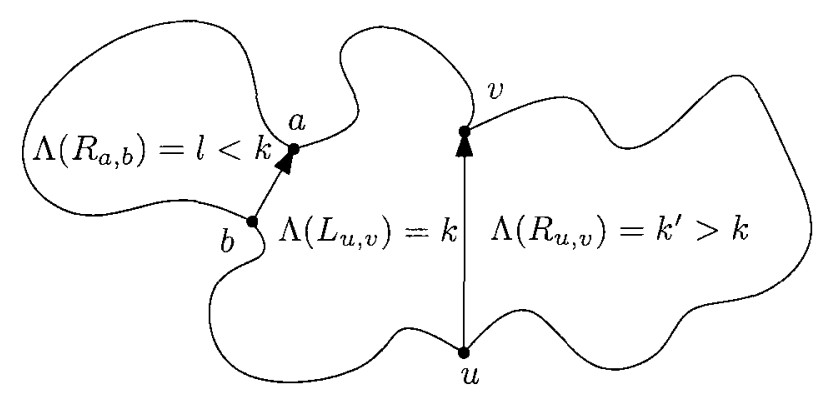

Figure 5.1: The figure shows how to find a directed path from $v$ to $u$ when $(u, v)$ is in $D(G ; \Lambda)$. If $\{a, b\}$ is any edge incident to $L_{u, v}$ then by inductive hypothesis there is a path from $a$ to $b$ of length at most $(\Phi-1)^{l}$. 
Next we give the proof of the statement (i) above. Base step is $\Lambda\left(L_{u, v}\right)=1$. Hence, the other face incident to $\{u, v\}$ has color $j>1$. Therefore, $(u, v) \in E_{1, j}$ which is in $D(G ; \Lambda)$ by definition. We have the same conclusion for any other arc of the face $L_{u, v}$ and hence this face will induce a directed cycle in $D(G ; \Lambda)$, which provides a desired directed path from $v$ to $u$. The length of this path is obviously at most $\Phi(G)-1$. Also every arc of this path is obviously incident to a face of color 1 . This proves the base case.

In the inductive step we assume the statement is true for all $l \leq k-1$. We prove it for $k$. Assume $\Lambda\left(L_{u, v}\right)=k$. If $k<\Lambda\left(R_{u, v}\right)=k^{\prime}$, then $(u, v) \in E_{k, k^{\prime}}$ which is in $D(G ; \Lambda)$ by definition. To construct a directed path from $v$ to $u$, consider the face $L_{u, v}$ and any edge $\{a, b\}$ incident to this face so that $L_{a, b}=L_{u, v}$. If $\Lambda\left(L_{a, b}\right)<\Lambda\left(R_{a, b}\right)$, the $\operatorname{arc}(a, b)$ is in $D(G ; \Lambda)$, and $\Lambda\left(L_{a, b}\right)=k \leq k$ as required. Otherwise the $\operatorname{arc}(b, a)$ is in $D(G ; \Lambda)$ and also $l=\Lambda\left(L_{b, a}\right)=\Lambda\left(R_{a, b}\right)<\Lambda\left(L_{a, b}\right)=k$ (see Figure 5.1). Thus, by inductive hypothesis, there is a directed path from $a$ to $b$ in $D(G ; \Lambda)$ of length at most $(\Phi(G)-1)^{l}$ such that every arc of this path is incident to a face of color at most $\Lambda\left(L_{b, a}\right)=l \leq k$. At most $(\Phi(G)-1)$ arcs of $L_{u, v}$ will be replaced in this way, so the length of the desired path from $v$ to $u$ is at most $(\Phi(G)-1)^{k}$. If $k>\Lambda\left(R_{u, v}\right)=k^{\prime}$, then $(u, v)$ is not in $D(G ; \Lambda)$.

Finally we give the proof of the statement (ii) above. Base step is $\Lambda\left(R_{u, v}\right)=\lambda$ which is trivially true since $(v, u)$ is not in $D(G ; \Lambda)$. In the inductive step we assume the statement is true for all $l \geq \lambda-k+1$. We prove it for $\lambda-k$. Assume $\Lambda\left(R_{u, v}\right)=\lambda-k$. If $\lambda-k>$ $\Lambda\left(L_{u, v}\right)=k^{\prime}$, then $(u, v) \in E_{k^{\prime}, \lambda-k}$ which is in $D(G ; \Lambda)$ by definition and hence $(v, u)$ is not in $D(G ; \lambda)$. Hence suppose $\lambda-k<\Lambda\left(L_{u, v}\right)=k^{\prime}$. Hence $(v, u) \in E$. To construct a directed path from $u$ to $v$, consider the face $L_{u, v}$ and any edge $\{a, b\}$ incident to this face so that $R_{a, b}=L_{u, v}$. If $\Lambda\left(L_{a, b}\right)<\Lambda\left(R_{a, b}\right)$, the $\operatorname{arc}(a, b)$ is in $D(G ; \Lambda)$, and $\Lambda\left(L_{a, b}\right)=k^{\prime} \geq \lambda-k$ as required. Otherwise the $\operatorname{arc}(b, a)$ is in $D(G ; \Lambda)$ and also $\Lambda\left(R_{a, b}\right)=k^{\prime}$. Thus, by inductive hypothesis, there is a directed path from $a$ to $b$ in $D(G ; \Lambda)$ of length at most $(\Phi(G)-1)^{\lambda-k^{\prime}+1}$ such that every arc of this path is incident to a face of color at lest $\Lambda\left(R_{a, b}\right)=k^{\prime}>\lambda-k$. At most $(\Phi(G)-1)$ arcs of $L_{u, v}$ will be replaced in this way, so the length of the desired path from $u$ to $v$ is at most $(\Phi(G)-1)^{\lambda-k^{\prime}+1+1} \leq(\Phi(G)-1)^{k+1}$, since $k^{\prime} \geq \lambda-k+1$. This proves the theorem.

As a corollary we obtain the following result on triangulations.

Corollary 25. Let $G=(V, E, F)$ be a geometric planar triangulation, and let $\Lambda$ be its face 
4-coloring. The digraph $D(G ; \Lambda)$ is strongly connected, has exactly $3|V|-3-k$ arcs, and its hop stretch factor is at most $(k-1)^{3}$; where $k$ is the degree of the outer face.

\subsection{Orientations with less than $|E|$ arcs}

By considering more sophisticated face colorings, we can decrease the number of arcs below $|E|$ in a strong orientation and still maintain a bounded hop stretch factor. Define

$$
D^{\prime}(G ; \Lambda)=D(G ; \Lambda)-E_{\left\lceil\frac{\lambda-1}{2}\right\rceil,\left\lceil\frac{\lambda+1}{2}\right\rceil} .
$$

We use the following result about acyclic coloring of planar graphs. A (proper vertex) coloring is acyclic if every subgraph induced by any two colors is acyclic.

Theorem 26. [8] Every planar graph has an acyclic coloring with 5 colors.

Lemma 27. Let $T=(V, E)$ be a forest and $k \geq 1$ an integer. There exists a set of vertices $S \subseteq V$ such that the subgraph of $T$ induced by $S$ is a forest of trees of diameter at most $4 k$ and has at least $\frac{k}{k+1}|E|$ arcs.

Proof. In this proof all indices will be considered modulo $2 k+2$. Root every component of $T$ at any vertex and consider the partition of $V$ into $k$ sets $V_{0}, V_{1}, \ldots, V_{2 k+1}$ such that the set

$$
V_{\ell}=\{x \in V: \text { distance of } x \text { from the root of its component is } \ell \bmod 2 k+2\} \text {. }
$$

Now consider the following $k$ forests of trees of diameter at most $4 k$. For $m=0,1, \ldots, k-$ 1 , let $G^{m}=\left(V^{m}, E^{m}\right)$ where

$$
\begin{aligned}
& V^{m}=V_{0-2 m} \cup V_{1-2 m} \cup \cdots \cup V_{2 k-2 m}, \\
& E^{m}=\left\{\{x, y\} \in E: x \in \cup_{i=0-2 m}^{2 k-1-2 m} V_{i}, y \in \cup_{i=1-2 m}^{2 k-2 m} V_{i}\right\} .
\end{aligned}
$$

It is not difficult to see that every $G^{m}$ is, in fact, induced subgraph of $T$. If one of the graphs $G^{m}$ has at least $\frac{k}{k+1}|E|$ arcs, we are done. On the other hand, only edges of $T$ that are not included in $G^{m}$ for given $m$ are edges $\{x, y\}$ such that $x \in V_{2 k-2 m}$ and $y \in V_{2 k+1-2 m}$ and edges $\{x, y\}$ such that $x \in V_{2 k+1-2 m}$ and $y \in V_{2 k+2-2 m}$, i.e. edges of stars centered at vertices in $V_{2 k+1-2 m}$. Since $G^{m}$ has less than $\frac{k}{k+1}|E|$ edges, there is at least $\frac{1}{k+1}|E|$ such 
edges. This must be true for all $m=0,1, \ldots, k-1$, and these edge sets are pairwise disjoint for distinct values of $m$. Hence the graph $G^{k}=\left(V^{k}, E^{k}\right)$ such that

$$
\begin{aligned}
& V^{k}=V_{2} \cup V_{3} \cup \cdots \cup V_{2 k+2}, \\
& E^{k}=\left\{\{x, y\} \in E: x \in \cup_{i=2}^{2 k+1} V_{i}, y \in \cup_{i=3}^{2 k+2} V_{i}\right\}
\end{aligned}
$$

is the forest of trees of diameter at most $4 k$, is induced in $T$ and has at least $\frac{k}{k+1}|E|$ edges.

The main theorem is as follows.

Theorem 28. Let $G(V, E, F)$ be a geometric planar graph which is 3-edge connected, and let $k \geq 1$ be an integer. There exists a face 6-coloring $\Lambda$ of $G$ so that the digraph $D^{\prime}(G ; \Lambda)$ is strongly connected, has at most $\left(1-\frac{k}{10(k+1)}\right)|E|$ arcs, and its hop stretch factor is at most $\Phi^{2}(G)(\Phi(G)-1)^{2 k+4}$.

Proof. Let $G^{\star}$ be the dual graph of $G$. Since $G$ is 3-edge connected $G^{\star}$ is a simple graph and every edge of $G$ is crossed by a unique edge of $G^{\star}$. Consider an acyclic 5-coloring of $G^{\star}$ which exists by Theorem 26. Among all ten pairs of colors in the 5-coloring choose a pair so that the forest $H$ induced by vertices colored with these two colors has at least $\frac{|E|}{10}$ edges. By Lemma 27, in this forest we can select a set of induced trees each of diameter at most $4 k$ such that they will together span at least $\frac{k}{k+1} \frac{|E|}{10}$ edges of $G^{\star}$.

We are now ready to color faces of $G$ and define a face 6-coloring $\Lambda$ of $G$ as follows: We use colors 3 and 4 to color faces corresponding to vertices of trees selected in the dual $G^{\star}$, and we use colors $1,2,5$, and 6 to properly color remaining faces of $G$.

With $\lambda=6$, we let color $\alpha=\left\lceil\frac{\lambda-1}{2}\right\rceil=3$ and $\beta=\left\lceil\frac{\lambda+1}{2}\right\rceil=4$. By our construction, the pair $\{\alpha, \beta\}$ must appear at least $\frac{k}{k+1} \frac{|E|}{10}$ times in the face 6-coloring of $G$. This gives the required bound on the number of arcs of the graph $D^{\prime}(G ; \Lambda)$.

Statements (i) and (ii) given in the proof of Theorem 24 imply that if $\{u, v\} \in E$ such that $\Lambda\left(L_{u, v}\right)<\Lambda\left(R_{u, v}\right)$ and if either $\Lambda\left(L_{u, v}\right) \neq \alpha$ or $\Lambda\left(R_{u, v}\right) \neq \beta$, then $D^{\prime}(G ; \Lambda)$ contains directed path from $u$ to $v$ as well as from $v$ to $u$. Indeed, if $\Lambda\left(L_{u, v}\right) \geq \alpha$, then $\Lambda\left(R_{u, v}\right)>\beta$, and we can apply statement (ii). Similarly if $\Lambda\left(R_{u, v}\right) \leq \beta$, then $\Lambda\left(L_{u, v}\right)<\alpha$, and we can apply statement (i). Obviously the pair of colors $\alpha$ and $\beta$ is not incident to any arc on these 
paths, so these paths exist in $D^{\prime}(G ; \Lambda)$. Note that these paths have bounded hop stretch factor as in Theorem 24 , in particular $(\Phi(G)-1)^{4}$.

To complete the proof we consider $\{u, v\} \in E$ such that $\Lambda\left(L_{u, v}\right)<\Lambda\left(R_{u, v}\right)$ and $\Lambda\left(L_{u, v}\right)=$ $\alpha$ and $\Lambda\left(R_{u, v}\right)=\beta$. Hence these edges do not occur in $D^{\prime}(G ; \Lambda)$. The edge $\left\{L_{u, v}, R_{u, v}\right\}$ of the dual $G^{\star}$ belongs to one of the selected trees, say $T$, of diameter at most $4 k$. The vertices of this tree correspond to faces of $G$ that are colored with color 3 or 4 . Since $G$ is 3-edge connected, there is a path $P$ from $u$ to $v$ in $G$ along these faces such that for each edge of $P$ one of its incident faces has color different from 3 and 4 . Hence for each edge of $P$ the digraph $D^{\prime}(G ; \Lambda)$ contains directed paths (in both directions) of length at most $(\Phi(G)-1)^{4}$. Since the maximum degree of $T$ is $\Phi(G)$ and the diameter of $T$ is at most $4 k, T$ has at most $\Phi(G)(\Phi(G)-1)^{2 k}$ vertices. Each of these vertices corresponds to a face of degree at most $\Phi(G)$. Hence the length of $P$ is at most $\Phi^{2}(G)(\Phi(G)-1)^{2 k}$. Finally we conclude that $D^{\prime}(G ; \Lambda)$ contains a directed path from $u$ to $v$ and from $v$ to $u$ both of length at most $\Phi^{2}(G)(\Phi(G)-1)^{2 k+4}$. It follows that $D^{\prime}(G ; \Lambda)$ is strongly connected and has the hop stretch factor at most $\Phi^{2}(G)(\Phi(G)-1)^{2 k+4}$.

Note that with a more careful counting argument the bound on the hop stretch factor in Theorem 28 can be improved by at least half. Using the following theorem we can further decrease the number of arcs in a strong orientation of $G$ and still keep hop stretch factor bounded.

Theorem 29. Let $G=(V, E, F)$ be a 3-connected planar graph. Then $G$ contains a spanning 2-edge connected subgraph $G^{\prime}$ with at most $|E|-\left\lfloor\frac{|E|+3}{3 \Phi(G)}\right\rfloor$ edges and $\Phi\left(G^{\prime}\right) \leq$ $2(\Phi(G)-1)$.

Proof. Since $G$ is 3-connected, the dual $G^{\star}$ is a simple graph. Let $T$ be a spanning tree of $G^{\star}$. Obviously, the maximum degree of $T$ is at most $\Phi(G)$ and $T$ has least $\frac{|E|}{3}+2$ vertices. The later follows from Euler's formula and the fact that minimum degree of $G$ is 3. Moreover, $T$ has a matching of size at least $\left\lfloor\frac{|E|+3}{3 \Phi(G)}\right\rfloor$. Indeed, one can obtain such a matching $M$ by recursively adding a pendant edge (an edge adjacent to a leaf) of remaining components of $T$ into $M$ and then removing all remaining edges incident to this edge. Each such operation adds one edge into $M$ and removes at most $\Phi(G)$ edges (including the edge itself) from $T$. Since $T$ has $\frac{|E|}{3}+1$ edges at the beginning, the bound follows. 
To obtain $G^{\prime}$, we merge corresponding faces in $G$ for every edge in $M$. Since $M$ is a matching $\Phi\left(G^{\prime}\right) \leq 2(\Phi(G)-1)$ and $G^{\prime}$ will be 2 -connected. Obviously $G^{\prime}$ has required number of edges.

\subsection{Conclusion}

We presented algorithms for directing edges of a planar graph having no cut edges such that the resulting digraph is strongly connected and has bounded hop stretch factor which depends solely on the size of the faces of the original planar graph. An interesting question arises how to construct planar spanning graphs having no cut edges. Although it is wellknown how to construct such planar graphs starting from a set of points (e.g., Delaunay triangulation) there are no known constructions in the literature of "local" spanning graphs from UDGs which also guarantee planarity and 2-edge connectivity at the same time. In the following chapters, we will focus on answering this question. 


\section{Chapter 6}

\section{Length 3-Times Optimal, 2-Edge Augmentation of Planar Graphs}

\subsection{Introduction}

In several network applications it is desirable to construct a spanning subgraph of a given UDG with "robust connectivity", in the sense that the spanning subgraph remains connected under edge deletion. The main question arising is given a UDG on a set of points how to construct a $k$-edge (respectively, vertex) connected spanning graph respecting the UDG and that has good planarity and connectivity characteristics as well as edge lengths which are a constant multiple of the unit radius of the UDG. Given a planar spanning graph, we can augment its connectivity by adding new edges of bounded length. The main issues arising are the number of edges being added, as well as the length of the new edges (since these represent the ranges of the corresponding points) which should be bounded by a constant independent of the size of the network.

\subsubsection{Our Results}

In Section 6.2 we give bounds on the number of newly added straight-line edges and show that such edges can be of length at most three times the max length of the edges of the original graph. If the original graph is a tree with $n$ nodes and max degree $\Delta$ then we prove that at most $n(1-1 / 2 \Delta)$ edges are sufficient, while for MSTs at most $5 n / 6$ edges are shown to be sufficient. All these algorithms are linear in the number of points. In addition, in Subsection 6.2.2 we indicate how to extend the NP-Completeness proof of [61] in order to show that it is NP-Hard to augment a geometric planar graph to a 2-edge connected geometric planar graph with the minimum number of new edges of bounded length. In Section 6.3, we prove that there is no local algorithm (i.e., a distributed algorithm that finishes in constant time by using only information at constant distance assuming each link takes one time unit to traverse) for augmenting a planar UDG into a 2-edge connected 
planar graph with straight line edges. In Section 6.4, we present a constant time algorithm that works in location-aware settings to augment the connectivity of a planar graph $G$ into a 2-edge connected planar graph with straight line edges of length bounded by 3 times the longest edge of $G$.

\subsection{Augmentation with Bounded Length Edges}

In this section we consider the augmentation problem for planar graphs. First we give an upper bound on the number of edges for arbitrary planar graphs and then consider the special cases of MSTs and general geometric trees. We point out that a zig-zag path with $n$ vertices of a convex polygon [1] requires $\lfloor n / 2\rfloor$ additional edges to augment it into a two-edge connected planar graph with edges of bounded length as Figure 6.1 depicts.

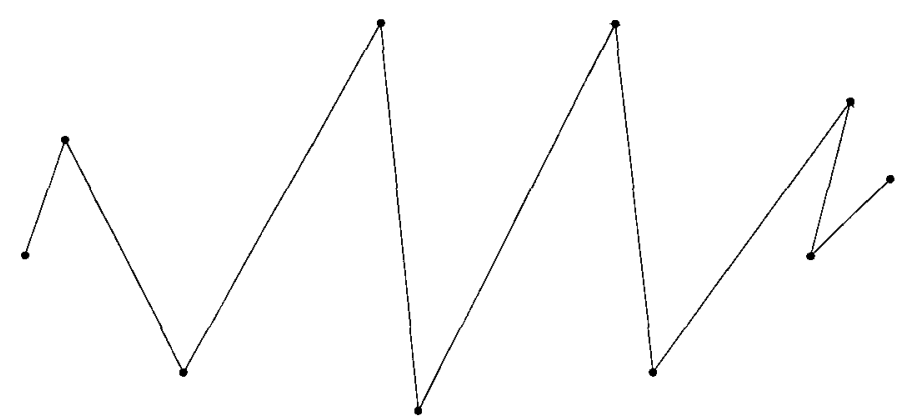

Figure 6.1: A graph of $n$ vertices that requires $\lfloor n / 2\rfloor$ new edges to augment it into a twoedge connected planar graph.

Recall that a two-edge connected planar graph $G=(V, E)$ remains connected after the removal of any edge. Therefore, every edge $\{u, v\} \in E$ is in at least one cycle. We say that $\{u, w\}$ is an immediate neighbour of $\{u, v\}$ if $\angle w u v<\pi$ and $u$ does not have any other neighbour inside the angle $\angle w u v$. Observe that an edge can have up to 4 immediate neighbours.

\subsubsection{Upper bounds}

Theorem 30. Let $G=(V, E)$ be a connected geometric graph with $|V| \geq 3$, vertices in general position in the plane (no three points are collinear), b cut edges and maximum edge length 1. Then, $G$ can be augmented to a 2-edge connected planar graph $G^{\prime}=$ 
$\left(V, E \cup E^{\prime}\right)$ with at most $b$ additional edges of length at most 3 times the longest edge of $G$ in time $O(|V|)$.

Proof. In the proof below we indicate how to add new edges leading to the construction of the new graph $G^{\prime}$. First, we introduce some notation that we require for the proof. We classify edges of $G^{\prime}$ in the following categories:

- $E_{1}$. All the original edges of $G$ or additional edges of length at most one. Thus, $E \subseteq E_{1}$.

- $E_{2}$. Additional edges of length at most two.

- $E_{3}$. Additional edges of length at most three.

The proof is constructive and in each step an additional edge of length at most three is added to create a cycle which includes at least one cut edge of $G$. The intuitive idea is to create a cycle by joining one cut edge $e$ with one of its immediate neighbours called the pivot of $e$ which in turn forms a triangle. Thus, an additional edge has always associated exactly one cut edge in $E_{1}$. We present the details in Algorithm 2.

The invariant throughout the proof is that additional edges belong to at least one cycle of $G^{\prime}$ having length at most three and each edge $e \in E_{3}$ is always incident in its triangle to one edge in $E_{2}$. Moreover, each additional edge is incident to an original cut edge.

Let $e=\{u, v\} \in E$ be any cut edge of $G^{\prime}$. (If it exists, otherwise $G^{\prime}$ is already two-edge connected planar graph.) Let $e_{1}=\{u, w\}$ be the immediate neighbour of $e$ in $G^{\prime}$ with min length such that if $e_{1} \notin E_{1}$ then $e_{1}$ is in a sector formed by the angle between $e$ and an immediate neighbour of $e$ in $G$, i.e., in a convex sector formed by original consecutive edges. $e_{1}$ exists since $|V| \geq 3$. Thus, a priority is given to the pivot $e_{1}$ in the following order: $E_{1}, E_{2}$ and $E_{3}$ (we refer this as priority order). Three cases can occur:

Case $1 e_{1} \in E_{1}$. Consider the triangle $w u v$, if it is empty, add $\{v, w\} \in E_{2}$ to $G^{\prime}$ and form a cycle with $e_{1}$ and $e$; see Figure 6.2a. Otherwise, there must exist a vertex $x$ inside the triangle $w v u$ such that the triangle $x v u$ is empty. (If there exist more than one then choose the closest to $u$.) Consider the two components of $G-e$. If $v$ and $x$ are in the same component, then add $\{u, x\} \in E_{1}$ to $G^{\prime}$; see Figure 6.2b. This creates a cycle with $\{u, v\}$. Otherwise, add $\{x, v\} \in E_{2}$ to $G^{\prime}$; see Figure 6.2c. This creates a cycle with $e$. 


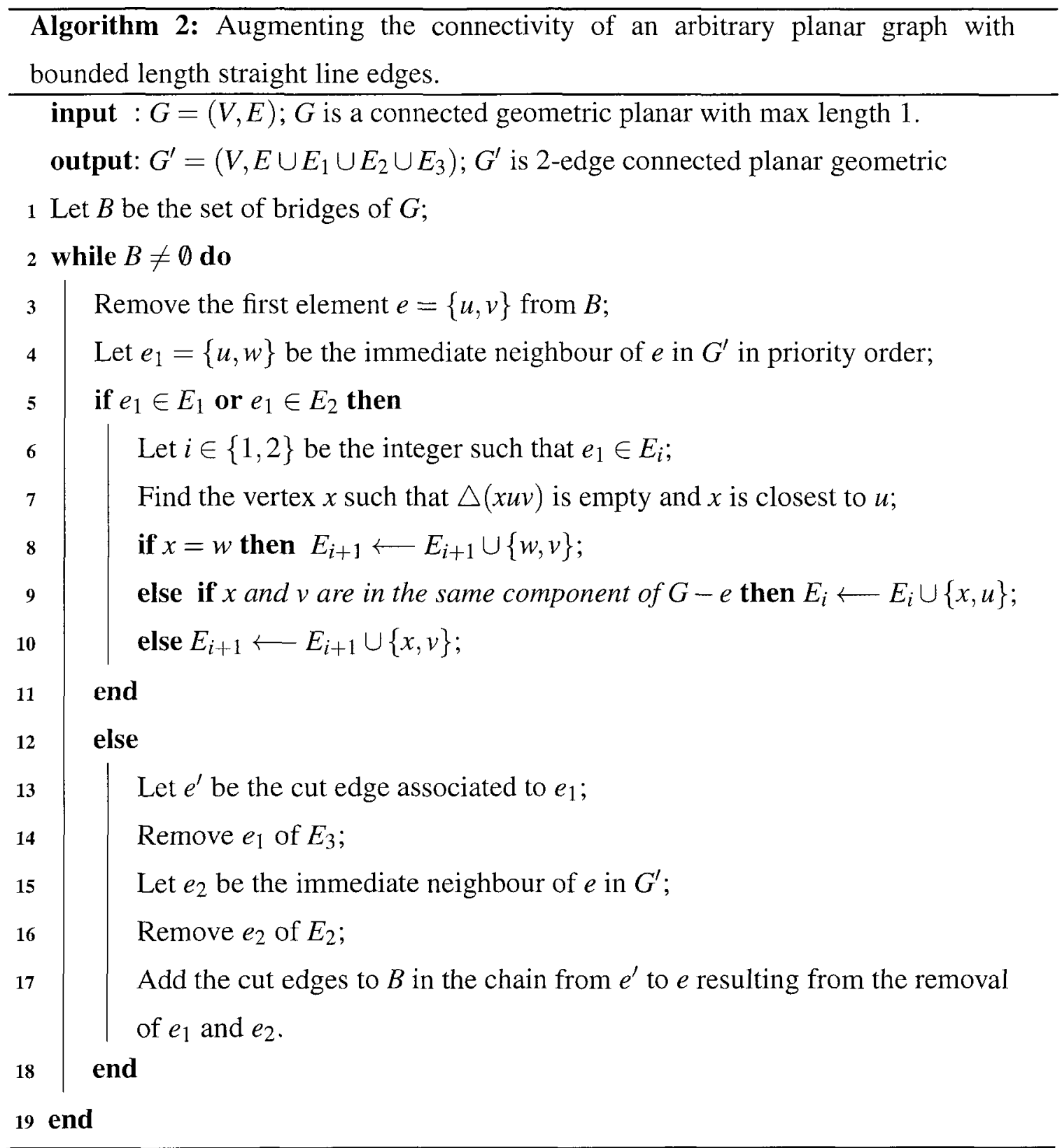

Thus, exactly one edge is added to $G^{\prime}$. Observe that any cut edge $e^{\prime}$ incident to $x$ (if it exists) will never choose $\{x, v\}$ as its pivot since $\{x, v\}$ is in a concave angle formed by two consecutive neighbours of $e^{\prime}$.

Case $2 e_{1} \in E_{2}$. This case only occurs when $e$ is either a leaf or when all its immediate neighbours in $G^{\prime}$ are in $E_{2}$. Similar to the previous case, consider the triangle wuv if it is empty, then add $\{w, v\} \in E_{3}$ to $G^{\prime}$. Otherwise, let $x$ be a vertex inside the triangle $w v u$ 


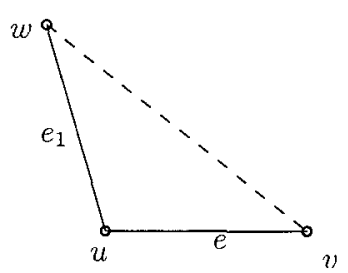

(a) wuv is empty.

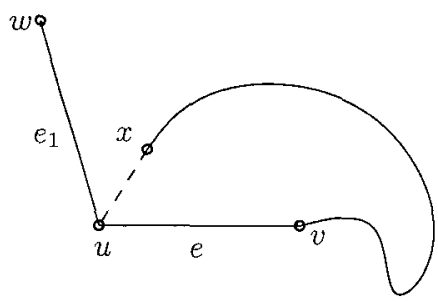

(b) $v$ and $x$ are in the same component.

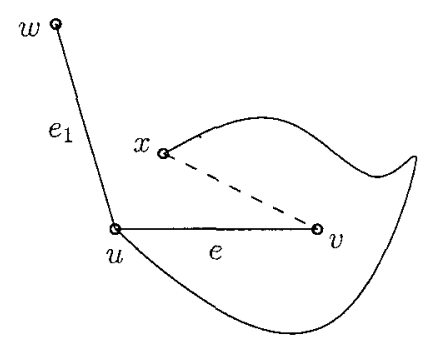

(c) $v$ and $x$ are in different components.

Figure 6.2: $e, e_{1} \in E_{1}$.

such that the triangle $x v u$ is empty (If there exist more than one then choose the closest to u.) Consider the two components of $G-e$. If $x$ and $v$ are in the same component, then add $\{u, x\} \in E_{2}$ to $G^{\prime}$. Otherwise, add $\{v, x\} \in E_{3}$ to $G^{\prime}$. In both cases $e$ is part of a cycle and exactly one edge is added to $G^{\prime}$. Similar to the previous case any cut edge $e^{\prime}$ incident to $x$ (if it exists) will never choose the new edge as its pivot since it is in a concave angle formed by two consecutive neighbours of $e^{\prime}$.

Case $3 e_{1} \in E_{3}$. This case also occurs only when $e$ is either a leaf or all its immediate neighbours in $G^{\prime}$ are in $E_{3}$. Since an edge of length greater than three is not allowed, some added edges must be removed to reconfigure $G^{\prime}$ and be able to employ the previous two cases. We will show that exactly two edge removals of previously added edges is always sufficient. Let $e^{\prime} \in E_{1}$ be the cut edge associated to $e_{1}$. Clearly $e^{\prime}$ exists and is not an immediate neighbor of $e$ in $G^{\prime}$, otherwise $e^{\prime}$ would have chosen $e$ as its pivot. Therefore, the third edge $e_{2} \in E_{2}$ of the triangle formed by $e_{1}$ and $e^{\prime}$ is incident to $e$. Similarly, let $e^{\prime \prime} \in E_{1}$ be the cut edge associated to $e_{2}$ which always exists. We will prove that $e^{\prime \prime}$ and $e_{2}$ form a triangle with another edge in $E_{1}$. If $e^{\prime \prime}$ is incident to $e$ then the third edge, say $e_{3}$, of the triangle formed by $e_{2}$ and $e^{\prime \prime}$ is also in $E_{1}$, otherwise $e^{\prime \prime}$ would have chosen $e$ as its pivot. On the other hand, if $e^{\prime \prime}$ is not incident to $e$ then $e^{\prime \prime}$ is incident to $e^{\prime}$ (otherwise $e^{\prime}$ would have never chosen $e_{2}$ as its pivot since $e_{2}$ would have been in a concave sector of $e^{\prime}$ ) and $e_{2}$ is incident to $e$ and $e_{3}$ and therefore $e_{3}$ is also in $E_{1}$. Thus, by removing $e_{2}$ and $e_{1}$ from $G^{\prime}$ and processing in order the cut edges in the chain from $e^{\prime}$ to $e$ will add edges only in $E_{1}$ and $E_{2}$; see Figure 6.3.

It is easy to see that the number of additional edges is at most $b$ since every additional edge is associated to exactly one cut edge. 


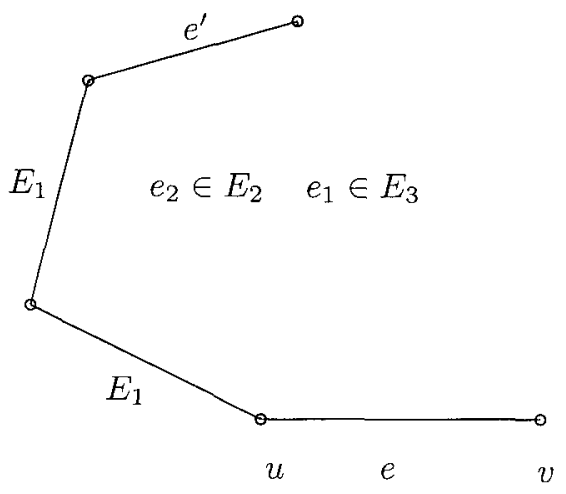

Figure 6.3: The removal of $e_{1}$ and $e_{2}$ leaves a chain of four edges in $E_{1}$.

The correctness of the Algorithm 2 comes from the proof above. Regarding the complexity, line 1 can be done in linear time by traversing the faces since there are $O(|V|)$ edges and each edge is visited twice. The while statement takes linear time in the number of bridges. Further, every step inside the while statement can be done in constant time by doing some preprocessing steps in linear time. Observe that the removal of added edges only affects processed cut edges at constant hop distance. Therefore, each cut edge is considered only a constant number of times. This completes the proof of Theorem 30 .

The upper bound 3 on the length of the augmented edges proved in Theorem 30 cannot be improved further as indicated by the example below.

Example 31. It is easy to see that edge length 3 is sometimes necessary for augmenting a planar graph to a 2-edge connected planar graph with straight line edges. Figure 6.4 depicts a graph indicating that this can be done only by adding the new edge $\{u, x\}$ of length $3-\varepsilon$. It is easy to see that any other possibility will create a crossing and therefore create a non-planar graph.

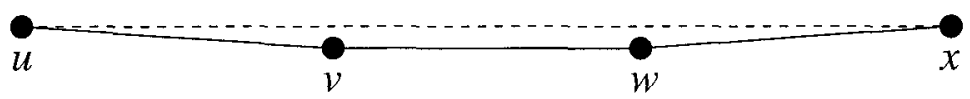

Figure 6.4: A planar graph that can be augmented to a 2-edge connected planar graph only by adding an edge of length $3-\varepsilon$.

Example 32. If we allow crossings, the planar graph depicted in Figure 6.5 can be augmented to a 2-edge connected graph by adding two edges of length $2-\varepsilon$. 


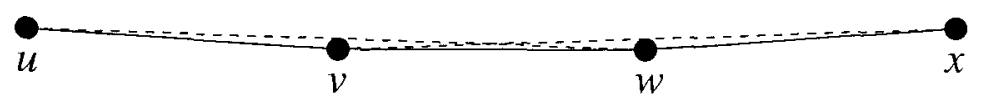

Figure 6.5: If we allow crossings, it can be augmented to a 2-edge connected graph by adding two edges of length $2-\varepsilon$.

Theorem 33. Let $T$ be an Euclidean MST on a set $P$ of $n$ points in the plane, $|P| \geq 3$ in general position. Then, it can be augmented to a 2-edge connected planar graph $G$ with at most $\lfloor 5 n / 6\rfloor$ additional edges of length at most 3 times the maximum edge length of $T$ in $O(n)$ time.

Proof. Let $T$ be an Euclidean MST and $l$ be its number of leaves. Recall that the maximum degree of $T$ is bounded by the kissing number in 2D, i.e., 6. Observe that in any MST, two consecutive vertices $v, w$ with common neighbour $u$ form an empty triangle $u v w$. Coloring the vertices of $T$ with two colours, say 1 and 2 . Let $C$ be the set of vertices in the chromatic class, say 1 , with less number of leaves. It is easy to see that internal vertices of $T$ with color, say 1 , are stars with center of color 1 and leaves of color 2 . Further, a star with center of color 1 does not share any edge with any other stars with center of the same chromatic class. Let $S_{u}$ be a star with center $u \in C$ and $u_{0}, u_{1}, \ldots, u_{d(u)-1}$ be the neighbours of $u$ in clockwise order around $u$ such that the widest angle is formed by $u_{d(u)-1}$ and $u_{0}$. If $d(u)=2 k$, let

$$
S_{u}^{\prime}=S_{u} \cup \bigcup_{i=0}^{k-1}\left\{u_{2 l}, u_{2 l+1}\right\}
$$

Otherwise, if $d(u)=2 k+1$, let

$$
S_{u}^{\prime}=S_{u} \cup \bigcup_{l=0}^{k-1}\left\{u_{2 l}, u_{2 l+1}\right\} \cup\left\{u_{2(k-1)+1}, u_{2 k}\right\}
$$

Let

$$
G^{\prime}=T \cup \bigcup_{\forall u \in C} S_{u}^{\prime}
$$

Observe that $G^{\prime}$ has at most $\lfloor l / 2\rfloor$ cut edges which correspond to leaves of $T$. By Theorem $30,\lfloor l / 2\rfloor$ additional edges are enough to augment $G$ into a 2-edge connected planar graph. 
Let $V_{l}$ denote the number of vertices in $C$ of degree $i$. Thus, $V_{1}=l / 2 \leq n / 2$. Observe that

$$
n-1=\sum_{\forall u \in C} d(u)=\sum_{l=1}^{6} i V_{l} .
$$

Therefore, the number of additional edges is

$$
\begin{aligned}
V_{1}+V_{2}+2 V_{3}+2 V_{4}+3 V_{5}+3 V_{6} & \leq V_{1}+\frac{2}{3}\left(n-1-V_{1}\right) \\
& \leq \frac{V_{1}}{3}+\frac{2}{3}(n-1) \\
& <\frac{n}{6}+\frac{2 n}{3} \\
& =\frac{5 n}{6}
\end{aligned}
$$

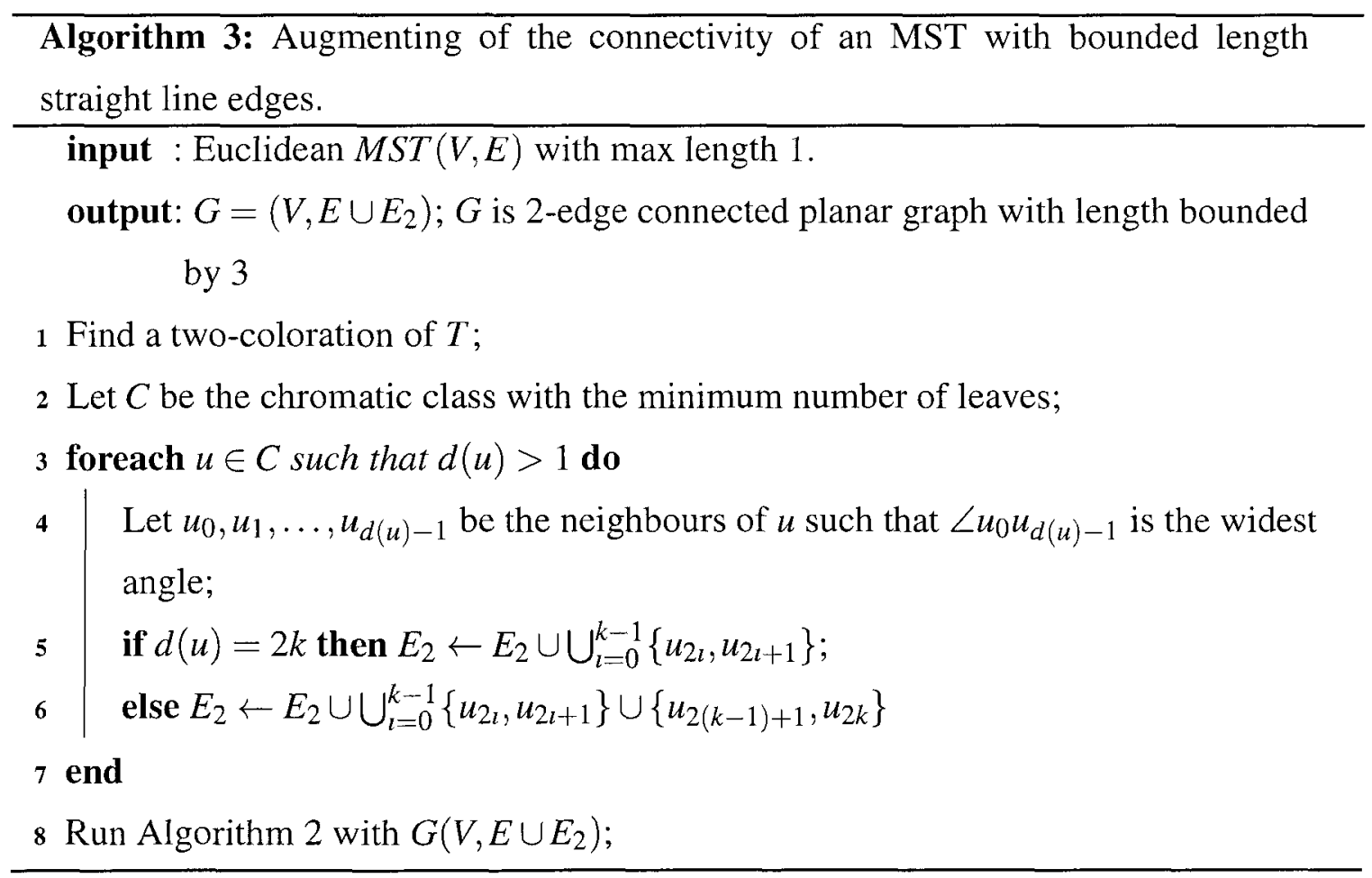

The pseudocode is presented in Algorithm 3. The correctness of Algorithm 3 comes from the proof above and the running time is easily seen to be linear in the number of vertices. This completes the proof of Theorem 33. 
Theorem 34. Let $T$ be any arbitrary planar tree $T$, with $n \geq 3$ vertices in general position and max degree $\Delta$ Then, it can be augmented to a 2-edge connected planar graph $G$ with at most $n\left(1-\frac{1}{2 \Delta}\right)$ additional edges of length at most 3 times the maximum edge length of Tin time $O(n)$

Proof Color vertices of $T$ with two colours, say 1 and 2 Let $C$ be the set of vertices in the chromatic class with the minimum number of leaves, say 1 Each internal vertex of $C$ is a star with center of color 1 and leaves of color 2 Moreover, stars with center in $C$ do not have any common edge However, a triangle formed with two consecutive vertices of a vertex $u$ is not necessarily empty We will show how to create cycles for each independent star of $C$ Let $u$ be any vertex in $C$ such that $d(u) \geq 2$ and $u_{0}$ and $u_{1}$ be two consecutive neighbours of $u$ forming an angle less than $\pi$ Consider triangle $u u_{0} u_{1}$ If it is empty, then add a cycle with $\left\{u_{0}, u_{1}\right\}$ Otherwise, consider the concave region of all the vertices inside the triangle $u u_{0} u_{1}$ including $u_{0}$ and $u_{1}$ There must ex1st two adjacent vertices $v$ and $w$ in the concave region such that $v$ and $w$ are in different components of $T \backslash u$ Moreover, by Theorem 30 at most $d-2$ additional edges are enough to create cycles with the remaining $d-2$ neighbours of $u$ Thus, each star with center $u$ can be augmented with $d(u)-1$ additional edges The Algorithm 4 summarizes this process

Let $V_{l}$ denote the number of vertices in $C$ of degree $l$ It is easy to see that $n-1=$ $\sum_{\forall u \in C} d(u)=\sum_{l=1}^{\Delta} l V_{l}$ Hence, the number of additional edges is

$$
\begin{aligned}
V_{1}+\sum_{l=2}^{\Delta}(l-1) V_{l} & \leq V_{1}+\frac{\Delta-1}{\Delta} \sum_{i=2}^{\Delta} l V_{l} \\
& =V_{1}+\frac{\Delta-1}{\Delta}\left(n-1-V_{1}\right) \\
& \leq l / 2+\frac{\Delta-1}{\Delta}(n-1-l / 2) \\
& <n / 2+\frac{\Delta-1}{\Delta}(n-1-n / 2) \\
& \leq n\left(1-\frac{1}{2 \Delta}\right)
\end{aligned}
$$

Algorithm 4 can be implemented in linear tıme by traversing $T$ and creatıng a data structure The correctness comes from the proof above This proves Theorem 34 
Algorithm 4: Augmenting of the connectivity of a arbitrary tree with bounded length straight line edges.

input $: T(V, E) ; T$ is a tree embedded in the plane such that the max length is 1 .

output: $G=\left(V, E \cup E_{2}\right)$; $G$ is 2 -edge connected planar graph with length bounded by 3

1 Find a two-coloration of $T$;

2 Let $C$ be the chromatic class with the minimum number of leaves;

3 foreach $u \in C$ such that $d(u)>1$ do

$4 \quad$ Let $u_{0}, u_{1}$ two consecutive neighbours of $u$ such that $\angle u_{0} u u_{1}<\pi$;

5 Find two vertices $x, y$ inside the triangle $u_{0} u u_{1}$ such that $x$ and $y$ are in different component of $T-u$;

6 $E_{2} \leftarrow E_{2} \cup\{x, y\}$

7 end

8 Run Algorithm 2 with $G\left(V, E \cup E_{2}\right)$;

\subsubsection{NP Completeness}

In this subsection we prove that it is NP-Hard to augment a geometric planar graph to a 2-edge connected geometric planar with the minimum number of new edges of any given bounded length. The proof is in fact a simple modification of the proof given in [61] to ensure that new edges are always bounded. Below we indicate the theorem which is necessary in order to derive the result.

Theorem 35. Augmenting a geometric planar graph $G$ into 2-edge connected geometric planar graph with the minimum number of new straight line of length $r$ times the longest edge of $G$ is NP-Hard.

Proof. Take an instance of a planar 3-SAT and consider the construction of the graph $G$ given in [61][Theorem 1]. Let $l$ be the longest edge of $G$ and $c$ the longest candidate edge to be added. If $c>r l$, then add a cycle at any given arbitrary vertex in the convex hull of $G$ in such a way that it does not interfere with $G$ and has at least one edge of length $l^{\prime} \geq c / r$. Thus, every new edge will be bounded by $r l^{\prime}$. The theorem follows by applying the same arguments given in [61]. 


\subsection{Impossibility of Local Algorithm for Augmentation}

In this section we prove that there is no local algorithm for augmenting a planar UDG to a 2-edge connected planar graph. We prove the following theorem.

Theorem 36. There is a unit disk graph $G$ with n nodes located in the plane in such a way that the following hold.

(i) Any location oblivious distributed algorithm for augmenting $G$ into a 2-edge connected planar graph with straight line edges requires $\Omega\left(\log ^{*} n\right)$ rounds.

(ii) There is a location oblivious distributed algorithm which takes $O\left(\log ^{*} n\right)$ rounds for augmenting $G$ into a 2-edge connected planar graph with straight line edges of length bounded by 3 times the longest edge.

In particular, such a planar augmentation of $G$ cannot be done locally.

Proof. Consider the unit disk graph depicted in Figure 6.6. Nodes are placed in the plane in such a way that for each node $i(0<i<n)$, the angle $\angle((i-1) i(i+1))$ formed by node $i$ with its two neighbours $i-1$ and $i+1$ is less than $\pi$. Observe that as a consequence of this geometric representation of the graph, for $i<j$ and $i^{\prime}<j^{\prime}$ the straight lines joining vertices $i, j$ and $i^{\prime}, j^{\prime}$ intersect if either $i<i^{\prime}<j$ or $i^{\prime}<i<j^{\prime}$. The vertices form a convex line graph and have arbitrary distinct identities, namely for all $i$, the $i$-th node has identity $i d_{l}$, for $i=0,1, \ldots, n-1$. Further, identifiers are picked from the range $\{1, \ldots, n\}$ and form an arbitrary permutation of this set.
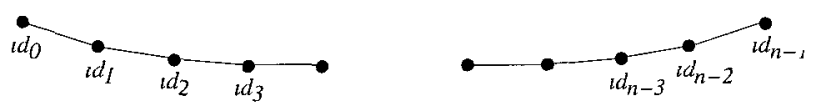

Figure 6.6: $n$ nodes arranged in a convex line graph. For each $i$, node $i$ has identity $l d_{l}$.

We assume a standard distributed computing model (see [55]) whereby every node has two ports (one for each of its two neighbours) except for the two endpoint nodes $i d_{0}, i d_{n-1}$ which have only one port and assume that they are consistently oriented to form the communication model of a convex line graph.

The proof of the first statement of the theorem is by contradiction to the fact the coloration problem takes $\Omega\left(\log ^{*} n\right.$ ) rounds (see [55][page 89]). Assume there is a distributed 
algorithm, say $\mathcal{A}$, for augmentation of the graph into a 2-edge connected planar graph terminating in $T$ rounds. Consider $n$ consecutive points on a convex line. In order to form a planar 2-edge connected graph, new edges are added to the convex line graph. This is done by informing each vertex $u$ of the vertex $u^{\prime}$ with which it forms a new edge. We call to such vertices a pair. (Note that some vertices may not be paired with any other vertex). Therefore by time $T$ and after execution of the algorithm $\mathcal{A}$ every node $u$ either

(i) is paired with another node $u^{\prime}$ so that $\left\{u, u^{\prime}\right\}$ forms a new edge, or

(ii) it is not paired with any other node of the line graph,

but the graph resulting by augmenting the line graph with the new pairs $\left\{u, u^{\prime}\right\}$ is planar and 2-edge connected.

Observe that all the new edges of the augmented planar graph are above the line graph as Figure 6.7 depicts. It is clear that $u$ and $u^{\prime}$ of each pair $\left\{u, u^{\prime}\right\}$ are at distance at most $T$ in the line graph since the running time of the algorithm is $T$ and a message takes one time unit to traverse an edge. Now we can give an algorithm for coloring the vertices of the graph that has running time $O(T)$. The algorithm is in two phases.

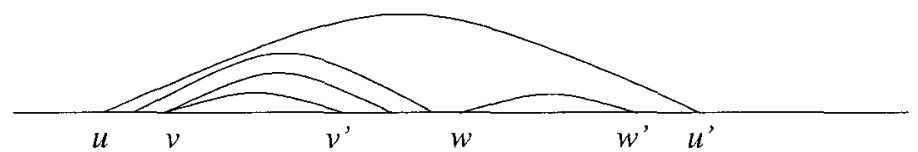

Figure 6.7: Pairing nodes of the line graph so as to form a 2-edge connected planar augmentation.

Phase 1: Maximum Intervals Discovery Algorithm. In Phase 1, we calculate maximum intervals between paired nodes as follows. In executing the algorithm $\mathcal{A}$ nodes can remember the port to which they received the messages. Therefore it is easy to see that if nodes $v, v^{\prime}$ are paired then they can execute an interval algorithm to discover the identities of all the nodes in the interval $I\left(v, v^{\prime}\right)$ delimited by the nodes $v, v^{\prime}$ in the line graphs. Clearly, the running time of this discovery algorithm is $T$. Further, every node $v$ which is paired with node $v^{\prime}$ runs an additional maximal interval algorithm to discover the maximum interval $I_{u, u^{\prime}}$ such that $I_{v, v^{\prime}} \subseteq I_{u, u^{\prime}}$. It is clear that since the augmented graph is planar 
this maximum interval is unique and well-defined. Moreover, the length of the maximum interval $I_{u, u^{\prime}}$ is at most $T$ since the running time of the algorithm $\mathcal{A}$ is $T$.

Phase 2: Coloring Algorithm. This Phase is executed only by pairs $u, u^{\prime}$ of nodes whose interval $I_{u, u^{\prime}}$ is maximum. First of all such nodes receive the same color, say $a$. Both $u, u^{\prime}$ can easily remember the sequence of identities as they are being received in their ports and color the nodes in the interval $I_{u, u^{\prime}}$ consistently using exactly two colors, say $b, c$. Clearly, the resulting coloring is consistent and has three colors. However, it is well known that any deterministic algorithm for 3 coloring a ring of $n$ nodes requires $\Omega\left(\log ^{*} n\right)$ rounds. Therefore $T \in \Omega\left(\log ^{*} n\right)$, as desired. This completes the proof of Part (ii).

To prove the second statement of the theorem we give an algorithm that augments $G$ into a 2-edge connected planar graph with straight line edges that runs in $O\left(\log ^{*} n\right)$ rounds. Consider a maximal independent set $I$ of $G$. Observe that two vertices in $I$ are separated by at most two vertices, otherwise $I$ is not maximal. We may assume that the end points of $G$ are in $I$, since we can include them into $I$ with a constant number of rounds. To see this, consider that the first point $v_{0}$ is not in $I$. Therefore, $v_{1} \in I$. Consider the second vertex $v_{j}$ in $I$. If $j=3$, then we can add $v_{0}$ to $I$ and remove $v_{1}$ from $I$. However, if $j=4$, then we can add $v_{0}$ and $v_{2}$ to $I$ and remove $v_{1}$ from $I$. Observe that after this process, $I$ is a valid maximal independent set. To create a 2 -edge connected planar graph it is enough to connect consecutive vertices in $I$. Clearly, the edges are bounded by 3 and the resulting graphs is planar. Since $I$ can be obtained in $O\left(\log ^{*} n\right)$ rounds [55] and every other steps can be done in a constant number of rounds, the construction takes $O\left(\log ^{*} n\right)$ rounds. This completes the proof of Theorem 36.

The impossibility theorem just proved hinges on the fact that edges are drawn as straight lines. If we drop this requirement then we can show that a local algorithm is possible even for augmentation to a 2 -vertex connected planar spanning graph.

Theorem 37. There is a location oblivious distributed, local algorithm which takes two rounds for augmenting a line graph into a 2-vertex connected, planar (non-geometric) graph. 
Proof. To prove the theorem, consider the case whereby the vertices form a line graph and have arbitrary distinct identities, namely for all $i$, the $i$-th node has identity $i d_{i}$, for $i=0,1, \ldots, n-1$. Further, identifiers are picked from the range $\{1, \ldots, n\}$ and form an arbitrary permutation of this set (see [55]). Now we give the local algorithm. Each node forms two new edges with nodes two hops away from it (one, if it is a node of degree 1 at an endpoint). In particular, for each $i \geq 2$ the following edges $\{i, i+2\},\{i-2, i\}$ are formed. The resulting graph has a planar representation (as depicted in Figure 6.8 by appropriately drawing the edges above and below the line) and it is also 2-vertex connected. This completes the proof of Theorem 37.

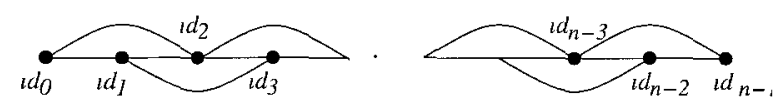

Figure 6.8: Planar representation of the line graph augmented with new edges.

Observe that the proof of Theorem 37 was based on an underlying line graph. Moreover the newly added edges that were used to form the augmented graph were not straight lines. We note that augmentation of a planar graph to a 2-vertex connected planar graph may not even be possible, in general. This is easily seen from the unit disk graph depicted in Figure 6.6. Clearly, a 2-vertex connected planar spanning graph is possible only by connecting the two endpoints $0, n-1$ with a straight line edge, which requires $n$ communication steps.

Example 38. The impossibility result given in Theorem 36 is also due to the fact that the identities are not ordered. For example, if $i d_{i}=i$, where $i=0,1, \ldots, n-1$, then the planar augmentation problem is easy. E.g., execute a distributed algorithm that draws new edges between nodes with even identities. This algorithm clearly works if $n$ is odd. If $n$ is even then the augmented graph resulting after execution of this algorithm will not have the rightmost edge $\{n-2, n-1\}$ in a cycle. For this reason we add instead the edge $\{n-3, n-1\}$ which is of length 3 . 


\subsection{Constant Time Augmentation in Location Aware Settings}

In this section we adopt the location-aware computation model of [14] and [43]. This setting allows us to determine the proximity neighbors in constant time by exchanging position information.

Theorem 39. Let $G=(S, E)$ be a graph on a set of points $S$. Then, there exists a locationaware algorithm that augments $G$ in constant time into a 2-edge connected planar graph with straight line edges of length bounded by 3 times the longest edge of $G$.

Proof. Let $v$ be a vertex in $G$. Consider the following classification of $v$ : If $d(v) \geq 2$ and it has two consecutive neighbors $u, w$ such that $\angle(u v w)<\pi$, then $v$ is an internal vertex of a convex line with respect to $u$ and $w$. If $d(v)=1$, then it is an end point of a convex line graph. Observe that $v$ is in at least one convex line graph. Further, a convex line graph may not have end points, i.e., if it is a convex face. An example is depicted in Figure 6.9.

New edges will be added to $E^{\prime}$. Let $C_{i}$ be a convex line graph of $G$ with at least one internal vertex. Consider a maximal independent set $I_{i}$ of $C_{i}$. Similar to the proof in Theorem 36, we may assume that the end points of $C_{i}$ (if it has) are in $I_{i}$ and $C_{i}$ has at least two vertices in $I_{i}$, otherwise $C_{i}$ is already a cycle of length 3 . Let $v_{a}$ and $v_{b}$ be two consecutive vertices in $I_{i}$ and $C H\left(I_{\left(v_{a}, v_{b}\right)}\right)$ be the convex hull of the interval $I_{\left(v_{a}, v_{b}\right)}$. If $C H\left(I_{\left(v_{a}, v_{b}\right)}\right)$ is empty, then let $E^{\prime}=E^{\prime} \cup\left\{\left\{v_{a}, v_{b}\right\}\right\}$. Otherwise, let $x$ and $y$ be the points inside $C H\left(I_{\left(v_{a}, v_{b}\right)}\right)$ such that $\angle\left(v_{a+1} v_{a} x\right)$ and $\angle\left(y v_{b} v_{b-1}\right)$ are minimum. Let $E^{\prime}=$ $E^{\prime} \cup\left\{\left\{v_{a}, x\right\},\left\{y, v_{b}\right\}\right\}$. Determining whether $C H\left(I_{\left(v_{a}, v_{b}\right)}\right)$ is empty or not can be done by exchanging information with nodes at a constant number of hops. Since every vertex is in at least one convex line graph, $G=\left(S, E \cup E^{\prime}\right)$ is 2-edge connected planar. Further, since $I_{\left(v_{a}, v_{b}\right)}$ has at most four vertices, the new edges are bounded by 3 .

An independent set in location-aware settings can be obtained in constant time [14], [73]. Hence, finding a maximal independent set in a convex line graph $C_{i}$ takes constant time and every other step also takes a constant number of rounds. Therefore, a 2-edge connected planar graph can be constructed in constant time. 


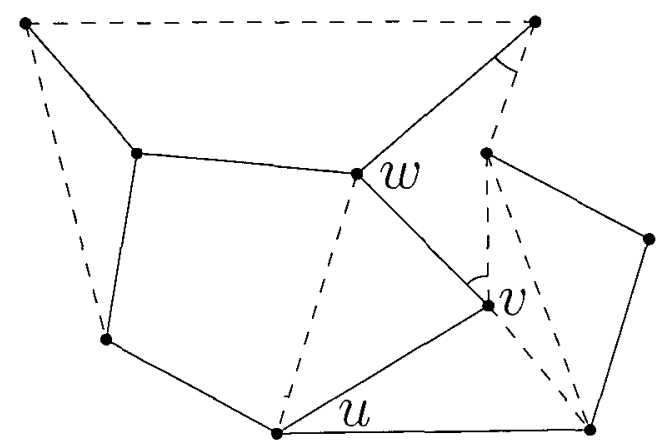

Figure 6.9: $v$ is an internal vertex of a convex line graph with respect to $u$ and $w$ and the end of two convex line graphs. (Solid lines represent original edges, dashed lines represent new added edges and dotted lines show the convex line graphs that the original graph forms.)

\subsection{Conclusion}

In this chapter we focused on the problem of constructing 2-edge connected geometric planar spanning graphs respecting an existing geometric graph. Such graphs are fault tolerant under edge deletion and because of their planarity can also be used to implement geometric routing with guaranteed delivery [43]. An interesting question arises when we ask for the minimum edge length necessary and sufficient to construct a 2-edge connected planar graph from a set of points. We study this problem in the next two chapters. 


\section{Chapter 7}

\section{Min Degree Planar Subgraphs}

\subsection{Introduction}

In this chapter we study the problem of finding planar subgraphs that do not have lowdegree nodes. More precisely, given a geometric graph $G=(V, E)$ and an integer $k$, we want to determine if $G$ has a planar spanning subgraph with the original embedding and straight-line edges such that all nodes have degree at least $k$.

This is a natural prerequisite in many problems related to communication networks. For example, if the answer is no, then certainly we cannot find a $k$-vertex connected or $k$-edge connected planar spanning subgraph, either. Moreover, if the answer is no, then we know that the domatic number [12], i.e., the number of disjoint dominating sets, of any planar spanning subgraph is at most $k$. On the positive side, if the answer is yes for $k=1$, then we can find a planar spanning subgraph that has domatic number at least 2 . For example, in a monitoring application, the two disjoint dominating sets can take turns in order to conserve energy.

The problem is easy to solve if $G$ is a complete graph and $k \leq 2$ : any triangulation of $V$ is a planar graph of degree at least 2, and hence the answer is yes if and only if we have at least $k+1$ nodes. The case of complete graphs and $k=3$ requires more thought, but it turns out that there is a planar spanning subgraph of minimum degree 3 iff we have at least 4 nodes and they are not in a convex position [3, 69]. Hence the problem can be solved in polynomial time for complete graphs and $k \leq 3$. On the other hand, for $k \geq 6$ the answer is always no: any planar graph contains a node of degree at most 5.

The problem is also easy to solve if $G$ is a connected UDG and $k=1$ : The answer is always yes, since the Euclidean minimum spanning tree in $G$ is planar. In this chapter we investigate what happens if we slightly deviate from the trivial case of UDGs and $k=1$. In particular, can we solve the problem efficiently in qUDG, which are relaxations of UDGs? Or can we solve the problem in other simple families of geometric graphs such as graphs 
with orthogonal edges? And what happens if $k=2$ or $k=3$ ? Surprisingly, it turns out that even the slightest deviations lead to NP-Complete decision problems.

The geometric $\alpha$-th power of a $G$, denoted by $G^{(\alpha)}$, the graph obtained from $G$ by adding all edges between vertices of (Euclidean) distance at most $\alpha \geq 1$.

\subsubsection{Our Results}

In Section 7.4 we study the case of minimum degree 1 . While the problem is trivial to solve in UDGs, we show that it is NP-Complete in a qUDG $(1-\varepsilon)$ for any positive constant $\varepsilon$. We also show that the problem is NP-Complete in orthogonal graphs. The case of $k=2$ is investigated in Section 7.5, and the case of $k=3$ in Section 7.6. In both cases it turns out that the problem is NP-Complete in UDGs.

As the strict decision problem turns out to be NP-Complete in the case of UDGs and $k \in\{2,3\}$, it is natural to ask whether we can solve the augmentation version of the problem with parameter $\alpha \geq 1$ : Either (i) prove that a given UDG $G$ does not have a planar

spanning subgraph of minimum degree $k$, or (ii) prove that $G^{(\alpha)}$ has a planar subgraph of minimum degree $k$. The case of $\alpha=1$ is equivalent to the original decision problem, and the case of $\alpha>1$ is strictly easier, as there are graphs for which both (i) and (ii) are the right answer. Our results in Sections 7.5 and 7.6 show that the case of $k \in\{2,3\}$ and $\alpha<\sqrt{5} / 2$ is NP-Hard, while the results presented in Chapter 6 implies that the case of $k=2$ can be solved in polynomial time.

The main new technique that we introduce in this chapter is the concept of planar circuit networks. In Section 7.2 we show that the problem of choosing an orientation of such networks is NP-Complete, and Sections 7.4-7.6 demonstrate that the orientation problem on planar circuit networks serves as a useful starting point in NP-Completeness proofs that are related to planar subgraphs.

\subsection{Orientation Problem on Planar Circuit Networks}

A planar circuit network is a planar geometric graph $\mathcal{C}=\left(V_{C}, E_{C}\right)$ with the following properties:

(i) The node set $V_{\mathcal{C}}=T_{C} \cup S_{\mathcal{C}} \cup U_{\mathcal{C}}$ consists of three disjoint subsets: terminals $T_{C}$, 
switches $S_{C}$, and users $U_{C}$.

(ii) The edge set $E_{\mathcal{C}}=P_{C} \cup W_{C}$ consists of two disjoint subsets: ports $P_{C}$ and wires $W_{C}$. Each port is labeled with either 1,2, or 3; the set of ports with label $x$ is denoted by $P_{C}(x)$.

(iii) Each wire joins a pair of terminals. Each port joins a terminal and a non-terminal.

(iv) Each terminal is incident to exactly 2 edges, and at least one of them is a wire.

(v) Each switch or user is incident to exactly 3 edges, and all of them are ports with different labels. That is, for each $v \in S_{C} \cup U_{C}$ and $x \in\{1,2,3\}$, there is exactly one edge in $P_{C}(x)$ that is incident to $v$.

Refer to Fig. 7.1 for an illustration; we draw terminals as black dots, switches as trapezoids with the port 1 on the short side, and users as squares. In what follows, we use the notation $v(x)$ to refer to the unique port with label $x \in\{1,2,3\}$ that is incident to $v \in S_{\mathcal{C}} \cup U_{\mathcal{C}}$.

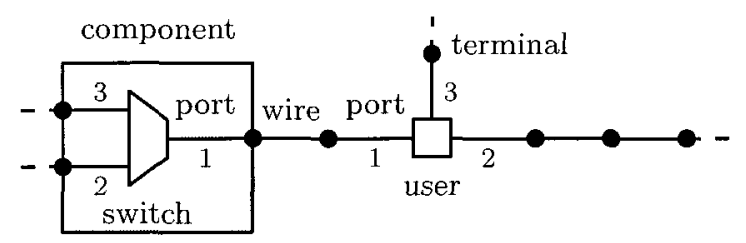

Figure 7.1: A circuit network.

We can partition a circuit network into components that are connected to each other by terminals. More precisely, a component consists of (i) a wire and two terminals or (ii) a user or a switch, three ports, and three terminals. Note that each terminal is contained in exactly two components. However, if $a$ is a wire, switch, or user, then there is a unique component $C[a]$ that contains $a$; in that case, we use the notation $a \rightarrow \bullet$ to denote that $t$ is a terminal in the component $C[a]$. We write $a \stackrel{x}{\bullet} t$ if the port that leads from switch or user $a$ to terminal $t$ has label $x$.

\subsection{Orientation Problem}

An orientation of a planar circuit network $\mathcal{C}$ assigns a direction to each edge $e \in E_{C}$. If $a-\bullet t$, and $t$ has indegree 1 in the component $C[a]$, then we write $a-\triangleright \bullet t$; otherwise $a \triangleleft \bullet \bullet$. If $a \stackrel{x}{\bullet} t$, we use notation $a \stackrel{x}{\bullet} t$ or $a \triangleleft \stackrel{x}{\bullet} t$. 
A valid orientation of a component $C[a]$ satisfies the following requirements (the first one is trivially satisfied but is listed here for reference):

(i) If $a$ is a wire, then there is at least one terminal $t$ with $a \triangleleft \bullet \bullet$.

(ii) If $a$ is a user, then there is at least one terminal $t$ with $a \triangleleft-\bullet t$.

(iii) If $a$ is a switch with $s \stackrel{2}{\sim} \bullet t_{2}$ or $s \stackrel{3}{\longrightarrow} \bullet t_{3}$, then $s \triangleleft \frac{1}{\bullet} t_{1}$.

A valid orientation of a circuit network $C$ satisfies the following additional requirement:

(iv) Each terminal has indegree at least one.

See Fig. 7.2 for an illustration. Not all circuit networks have valid orientations; in the orientation problem the task is to decide if a given planar circuit network $C$ has a valid orientation.

(a)

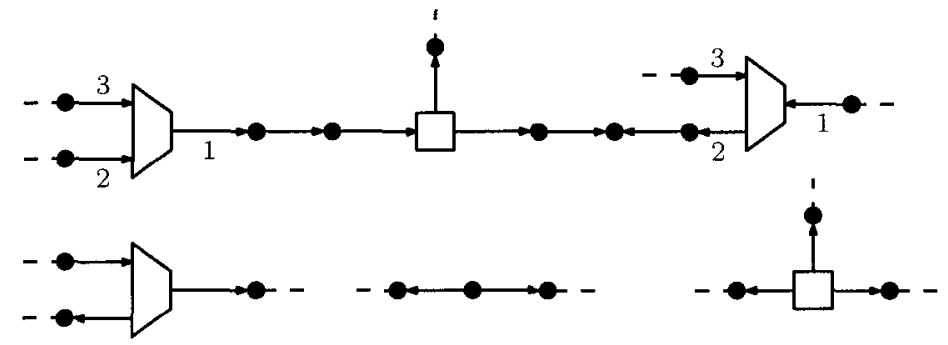

Figure 7.2: (a) Valid and (b) invalid orientations of a circuit network.

The following lemma shows that we can replace a path of wires by a single wire and vice versa, without affecting the essential properties of the problem.

Lemma 40. Let $C$ be a planar circuit network, and let $e=\{u, v\} \in W_{C}$ be a wire in C. Construct another planar circuit network $C^{\prime}$ by repeatedly subdividing $e$; that is, we replace $e$ by a path $P$ that consists of wires and terminals. Then if we are given a valid orientation of $\mathcal{C}$, we can find in polynomial time a valid orientation of $\mathcal{C}^{\prime}$, and vice versa.

Proof. Clearly if we are given a valid orientation of $C$, we can construct a valid orientation of $C^{\prime}$ as well. Now assume that we are given a valid orientation of $C^{\prime}$. Since all internal nodes of $P$ have indegree at least 1 , there must be at least one endpoint of $P$ that has indegree 0. W.l.o.g., we can assume that $u$ is an endpoint with indegree 0 . Then we can orient the wire $e$ in $C$ from $u$ to $v$. The orientations of all other edges are inherited from $C^{\prime}$. 
We will use the following theorem in reductions throughout this work. The theorem can be proved by a reduction from planar 3SAT.

Theorem 41. The orientation problem on planar circuit networks is NP-complete.

Proof. Clearly the orientation problem is in NP. We will show the NP-hardness by a reduction from the planar $3 S A T$ problem, which is known to be NP-complete [48].

\section{Planar 3SAT.}

An instance of the planar 3SAT problem is represented as a planar bipartite graph $\mathcal{S}=$ $\left(C_{\mathcal{S}} \cup X_{\mathcal{S}}, E_{\mathcal{S}}\right)$. Each $c \in C_{S}$ is a clause and each $x \in X_{\mathcal{S}}$ is a variable. Each edge $e \in E_{\mathcal{S}}$ joins a clause and a variable in the clause; a clause has degree at most 3 . Each edge is either positive or negative. A feasible solution is a satisfying truth assignment $f: X_{\mathcal{S}} \rightarrow\{0,1\}$ : for each clause $c \in C_{S}$ there is at least one edge $e=\{c, x\} \in E_{S}$ such that (i) $f(x)=1$ and $e$ is positive, or (ii) $f(x)=0$ and $e$ is negative.

\section{Reduction from Planar 3SAT.}

Given a 3SAT instance $\mathcal{S}$, we show how to construct a planar circuit network $\mathcal{C}$ with the following property: there exists a valid orientation of $\mathcal{C}$ if and only if there exists a satisfying truth assignment for $\mathcal{S}$. W.l.o.g., we can assume that each clause of $\mathcal{S}$ has degree equal to 3 (for example, we can replace a single edge by multiple parallel edges; our construction can be applied to planar multigraphs equally well).

To facilitate the construction, let us first choose an ordering of the endpoints of the edges in $\mathcal{S}$ by using the plane embedding of $\mathcal{S}$, as follows. For each node $v \in C_{\mathcal{S}} \cup X_{\mathcal{S}}$, start at an arbitrary edge incident to $v$ and label the incident edges in clockwise order by $e_{v}(1), e_{v}(2), \ldots, e_{v}(d)$, with $d=d(v)$. Hence we define a bijection $e_{v}$ from $I(v)=$ $\{1,2, \ldots, d(v)\}$ to the edges incident to $v$. 
The nodes and ports of the circuit network $C$ are defined as follows:

$$
\begin{aligned}
U_{C} & =\left\{\mu(c): c \in C_{S}\right\} . \\
S_{C} & =\left\{\sigma(x, i): x \in X_{\mathcal{S}}, i \in I(x)\right\}, \\
T_{C} & =\left\{\alpha(c, i): c \in C_{\mathcal{S}}, i \in I(c)\right\} \\
& \cup\left\{\beta(x, i, k): x \in X_{\mathcal{S}}, i \in I(x), k \in\{1,2,3\}\right\} \\
& \cup\left\{\gamma(x, i): x \in X_{\mathcal{S}}, i \in I(x)\right\}, \\
P_{C}(k) & =\left\{\{\mu(c), \alpha(c, k)\}: c \in C_{\mathcal{S}}\right\} \\
& \cup\left\{\{\sigma(x, i), \beta(c, i, k)\}: x \in X_{\mathcal{S}}, i \in I(x)\right\} .
\end{aligned}
$$

In particular, each switch $\sigma(x, i)$ and each user $\mu(c)$ is incident to 3 ports, each terminal $\alpha(c, i)$ is adjacent to one user $\mu(c)$, and each terminal $\beta(x, i, k)$ is adjacent to one switch $\sigma(x, i)$. So far the terminals $\gamma(x, i)$ are isolated nodes.

To complete the construction, we need to define the wires. To that end, consider an edge $e=\{x, c\} \in E_{\mathcal{S}}$ that joins a variable $x \in X_{\mathcal{S}}$ and a clause $c \in C_{\mathcal{S}}$; then there are unique integers $i \in I(x)$ and $j \in I(c)$ such that $e=e_{x}(i)=e_{c}(j)$. Let $i^{\prime} \in I(x)$ be such that $i^{\prime} \equiv i+1$ $(\bmod d(v))$. If $e$ is a positive edge, we add the following three wires to $W_{C}$ :

$$
\{\gamma(x, i), \beta(x, i, 1)\},\left\{\gamma\left(x, i^{\prime}\right), \beta(x, i, 2)\right\} \text {, and }\{\alpha(c, j), \beta(x, i, 3)\}
$$

However, if $e$ is a negative edge, we add the following three wires to $W_{C}$ :

$$
\left\{\gamma\left(x, i^{\prime}\right), \beta(x, i, 1)\right\},\{\gamma(x, i), \beta(x, i, 2)\} \text {, and }\{\alpha(c, j), \beta(x, i, 3)\} .
$$

This completes the construction. It follows that for each variable $x \in X_{S}$ there is a cycle $C(x)$ that consists of the $4 d(x)$ nodes $\gamma(x, i), \beta(x, i, 1), \beta(x, i, 2)$, and $\sigma(x, i)$ for $i \in I(x)$. Moreover, for each edge $e_{x}(i)=e_{c}(j) \in E_{S}$ there is a length-3 path $P(x, c)$ that connects $\sigma(x, i)$ to $\mu(c)$.

It can be checked that the resulting graph $\mathcal{C}$ is a valid circuit network. As $S$ is planar, we can easily find a planar embedding of $C$ as well: each clause $c$ is replaced by a node $\mu(c)$, each variable $x$ is replaced by a cycle $C(x)$, and each edge $\{x, c\}$ is replaced by a path $P(x, c)$ that joins the cycle $C(x)$ and the node $\mu(c)$. See Fig. 7.3 for an illustration. 

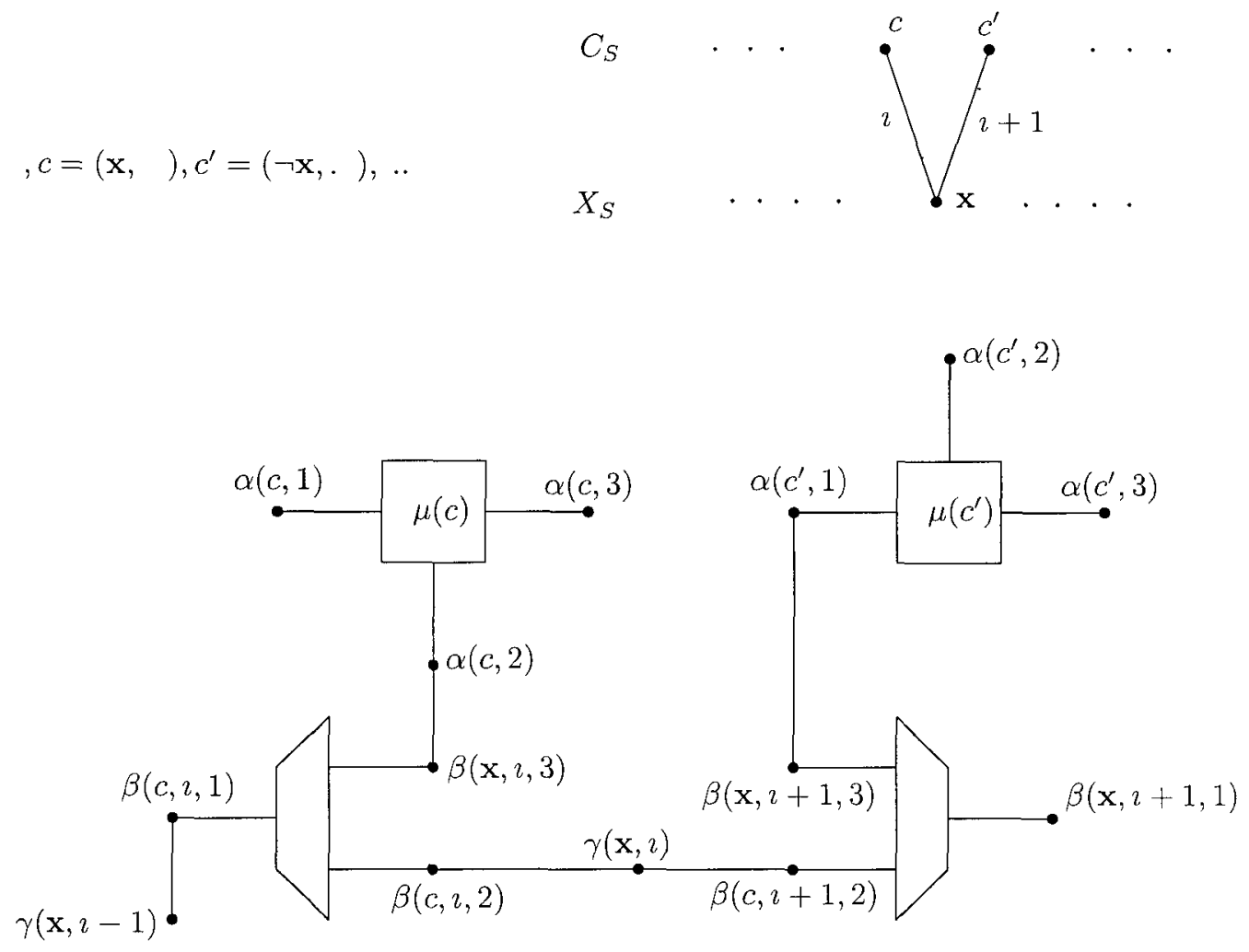

Figure 7.3: Connection between clauses and variables $\left(e_{x}(i)\right.$ is a positive edge and $e_{x}(i+1)$ is a negative edge).

\section{Normalized Orientations.}

The clockwise orientation of $C(x)$ is the directed cycle in which each length-4 subpath between $\gamma(x, i)$ and $\gamma\left(x, l^{\prime}\right)$ is oriented from $\gamma(x, i)$ to $\gamma\left(x, i^{\prime}\right)$. The counterclockwise orientation is the reversal of the clockwise orientation. The outwards orientation of $P(x, c)$ orients all edges from $\sigma(x, i)$ to $\mu(c)$. The inwards orientation is the reversal of the outwards orientation.

Consider any valid orientation of $\mathcal{C}$, and let $x \in X_{S}$. Observe that in the subgraph induced by $C(x)$, the indegree of each node must be at least 1 - recall that if port 2 of a switch is an outgoing edge, then port 1 cannot be an outgoing edge. Hence it follows that the indegree of each node has to be exactly 1 , and $C(x)$ is oriented either clockwise or counterclockwise. 
Moreover, let $i \in I(x)$ and consider the edge $e_{x}(i)=\{x, c\} \in E_{S}$. If the port $\{\sigma(x, i)$, $\beta(x, i, 3)\}$ is oriented from $\sigma(x, i)$ to $\beta(x, i, 3)$, then we can orient the whole path $P(x, c)$ outwards; otherwise we can orient the path $P(x, c)$ inwards. Such normalisations do not decrease the indegree of $\mu(c)$, they do not affect the orientation of the edges incident to $\sigma(x, i)$, and they maintain the requirement that the degree of each node along $P(x, c)$ is at least 1 ; indeed, it will be exactly one.

Hence for any valid orientation of $\mathcal{C}$, we can find a normalized orientation in which each $C(x)$ is oriented either clockwise or counterclockwise and each $P(x, c)$ is oriented either inwards or outwards.

\section{Equivalence of Normalized Orientations and Truth Assignments.}

First, consider a normalized orientation of $\mathcal{C}$. Then we can find a satisfying truth assignment $f$ of $S$ as follows: set $f(x)=1$ if and only if $C(x)$ is oriented clockwise. To see that this indeed satisfies each clause $c \in C_{S}$, observe that the indegree of $\mu(c)$ is at least one. Hence there is at least one $x$ such that the path $P(x, c)$ is oriented outwards. There are two cases:

(i) If $\{x, c\}$ is a positive edge, our construction implies that $C(x)$ must be oriented clockwise. We have set $f(x)=1$, and hence $f$ satisfies clause $c$.

(ii) If $\{x, c\}$ is a negative edge, it implies that $C(x)$ must be oriented counterclockwise. We have set $f(x)=0$, and hence $f$ satisfies clause $c$.

Second, consider a satisfying truth assignment $f$ of $S$. Then we can find a normalized orientation of $C$ as follows:

(i) For each variable $x \in X_{S}$ we orient $C(x)$ clockwise if and only if $f(x)=1$.

(ii) For each positive edge $\{x, c\} \in E_{S}$ we orient $P(x, c)$ outwards if and only if $f(x)=1$.

(iii) For each negative edge $\{x, c\} \in E_{\mathcal{S}}$ we orient $P(x, c)$ outwards if and only if $f(x)=0$.

It can be checked that this is indeed a valid orientation: in particular, each $\mu(c)$ has indegree at least one, and the switches are properly oriented. 
Hence the planar 3SAT instance $S$ is satisfiable if and only if there is a valid orientation of the planar circuit network $C$. This concludes the proof of Theorem 41 .

\subsection{Degree One}

In this section we study the problem of deciding if a given geometric graph has a planar spanning subgraph. The problem is trivial in UDG - a minimum spanning tree is a planar spanning subgraph. We will show that the problem is NP-complete in quasi unit disk graphs and in orthogonal graphs.

Theorem 42. The following problem is NP-complete for any $\varepsilon>0$ : given a graph $G \in$ qUDG $(1-\varepsilon)$, decide if $G$ has a planar spanning subgraph.

Proof. To prove the theorem, assume that we are given an $\varepsilon>0$ and a planar circuit network $C$. We will show how to construct a graph $G \in \mathrm{qUDG}(1-\varepsilon)$ with the following property: $G$ has a planar spanning subgraph if and only if $C$ has a valid orientation. The claim then follows by Theorem 41.

In our construction of $G$, we replace each component $\mathcal{C}[a]$ of $\mathcal{C}$ by a gadget $G[a]$ that implements the component. In the construction, we will have two kinds of nodes in $G$ : black nodes are identified with the terminals of $\mathcal{C}$, while grey nodes are internal to a gadget. A component with a wire is replaced with the gadget of Fig. 7.4a, a component with a switch is replaced with the gadget of Fig. $7.4 \mathrm{~b}$, and a component with a user is replaced with the gadget of Fig. 7.4c. Note that each black node is shared by exactly two gadgets.

Each gadget has very specific dimensions in order to guarantee that they are in qUDG $(1-\varepsilon)$; for example, in the wire gadget, the distance between the two terminals is $2-\varepsilon / 2$. Moreover, two gadgets must not be placed too close to each other; for example, an internal node of one gadget cannot be within distance $1-\varepsilon$ from the internal node of another gadget. Hence we cannot directly replace the components by gadgets in an arbitrary embedding of $\mathcal{C}$. However, we can always find an appropriate embedding by moving the components around and by exploiting the flexibility provided by Lemma 40; see Fig. 7.4d for an illustration of an appropriate embedding in the neighborhood of a switch. 
(a)

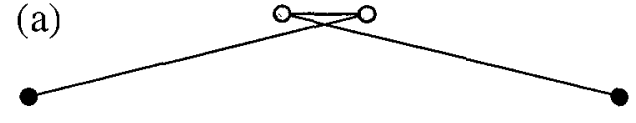

1

(c)

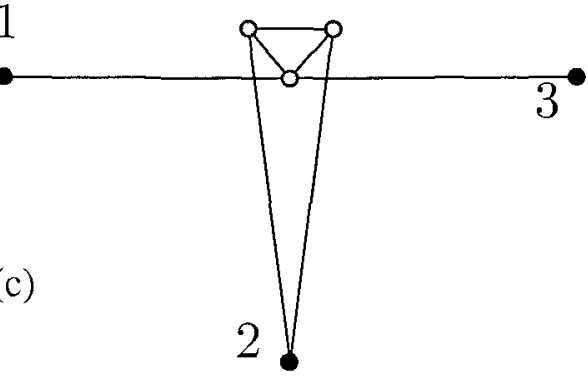

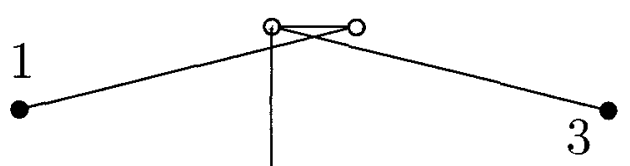

(b)
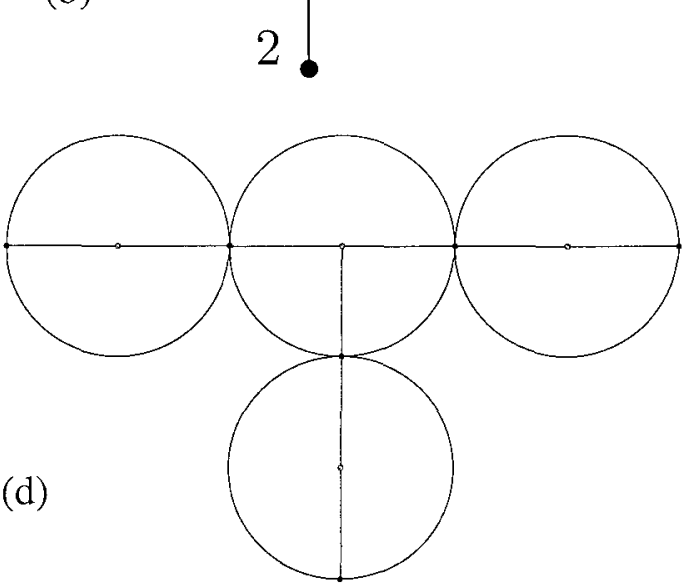

Figure 7.4: Gadgets for qUDG $(1-\varepsilon$ ) and minimum degree 1: (a) wire, (b) switch, (c) user. (d) Embedding of a switch, three ports, and three wires.

Now we proceed to relate the orientations of $C$ and the planar spanning subgraphs of $G$. Let us first focus on a component $\mathcal{C}[a]$ and the gadget $G[a]$ that implements it. We say that a subgraph $G^{\prime}[a]$ of $G[a]$ is internally good if $G^{\prime}[a]$ is planar and each grey node of $G[a]$ has degree at least one in $G^{\prime}[a]$. Note that if we have a planar spanning subgraph $G^{\prime}$ of $G$, then $G^{\prime}$ restricted to $G[a]$ is internally good. The key observation is summarized in the following lemma.

Lemma 43. Given a valid orientation of $C[a]$, we can find an internally good subgraph $G^{\prime}[a]$ such that $a \rightarrow \bullet t$ implies $d_{G^{\prime}[a]}(t) \geq 1$. Conversely, given an internally good subgraph $G^{\prime}[a]$, we can find a valid orientation of $C[a]$ such that $a-\bullet$ and $d_{G^{\prime}[a]}(t) \geq 1$ implies $a \rightarrow \bullet t$.

Proof. A straightforward case analysis.

Now if we are given a valid orientation of $\mathcal{C}$, we can apply Lemma 43 to each component $C[a]$ to find an internally good subgraph $G^{\prime}[a]$ for each $G[a]$; the union of $G^{\prime}[a]$ forms a subgraph $G^{\prime}$ of $G$. By construction, $G^{\prime}$ is planar and each grey node has degree at least one. It remains to be shown that each black node has degree at least one. To verify this, consider a terminal $t$. In a valid orientation, there is a component $C[a]$ such that $a \rightarrow t$. 
Hence in $G^{\prime}[a]$ we have $d_{G^{\prime}[a]}(t) \geq 1$, and therefore also $d_{G^{\prime}}(t) \geq 1$. Hence $G^{\prime}$ is a planar spanning subgraph of $G$.

Conversely, if we are given a planar spanning subgraph $G^{\prime}$ of $G$, we can apply Lemma 43 to each component $G[a]$ to orient $C$. We will have a valid orientation for each component; it remains to be shown that each terminal has indegree at least one. To verify this, consider a terminal $t$. Since $d_{G^{\prime}}(t) \geq 1$ we have a component $\mathcal{C}[a]$ such that $d_{G^{\prime}[a]}(t) \geq 1$, and hence an orientation with $a \rightarrow t$. Hence we have a valid orientation of $C$. This concludes the proof of Theorem 42 .

Theorem 44. The following problem is NP-complete: given an orthogonal graph $G$, decide if it has a planar spanning subgraph.

(a)

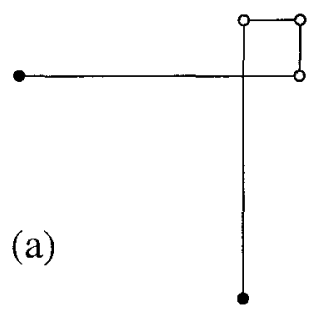

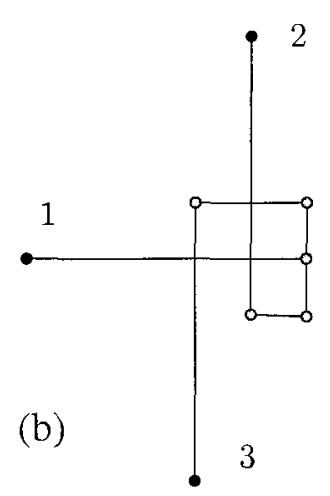

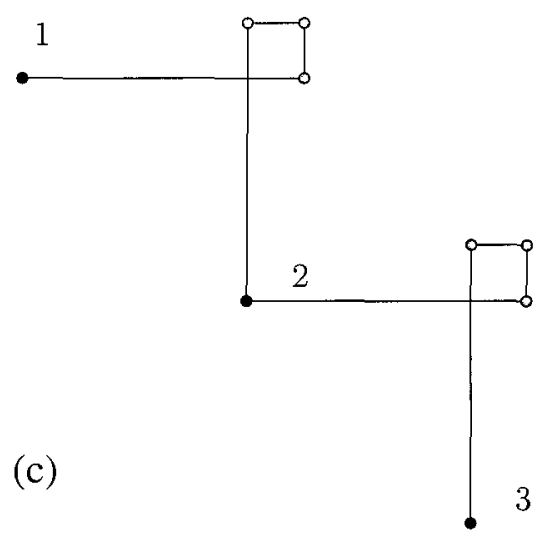

3

Figure 7.5: Gadgets for orthogonal graphs: (a) wire, (b) switch, (c) user.

Proof. The structure of the proof is identical to the proof of Theorem 42. We are only using a different set of gadgets: see Fig. 7.5.

\subsection{Degree Two}

In this section we study the case of planar spanning subgraphs that have degree at least 2 . It turns out that finding such subgraphs is NP-hard even in the case of UDG.

In what follows, we prove a stronger result by considering a variant in which we are allowed to augment the graph by adding edges of length $\alpha \geq 1$. In the augmentation 
problem we are allowed to return the answer that "the original graph does not have a planar spanning subgraph with minimum degree at least 2 " or "I do not know about the original graph, but if I first add all the edges between points at distance at most $\alpha, I$ can construct a planar spanning subgraph with minimum degree at least 2". Obviously, the case of $\alpha=1$ is equivalent to the original decision problem.

If $\alpha=3$, the problem can be solved in polynomial time by using techniques from Chapter 6: If we are given a graph $G \in U D G$, we can consider each connected component of $G$ separately. If a connected component contains fewer than 3 nodes, then we know that $G$ does not have a planar subgraph with minimum degree 2. Otherwise we can first find a spanning tree in each component, and then augment the trees by adding non-crossing edges of length at most 3 so that each connected component becomes 2-edge connected. In particular, after augmentation, the graph is planar and each node has minimum degree at least 2 .

In what follows, we prove that the problem is NP-complete if $\alpha<\sqrt{5} / 2 \approx 1.118$. We do this by formulating the problem as a promise problem.

Theorem 45. The following promise problem is $N P$-complete for any $1 \leq \alpha<\sqrt{5} / 2$ : given a graph $G \in U \mathrm{UDG}$, decide whether (i) there is no planar spanning subgraph of $G$ such that each node has degree at least 2 , or (ii) there is a planar spanning subgraph of $G^{(\alpha)}$ such that each node has degree at least 2.

Proof. The structure of the proof is similar to the proof of Theorem 42. For the sake of brevity, we only list the differences.

In Theorem 42 we constructed a graph $G \in$ qUDG $(1-\varepsilon)$; this time we construct a graph $G \in$ UDG. Moreover, our construction satisfies $G=G^{(\alpha)}$, that is, the augmentation does not change the graph at all, and hence the augmentation problem is exactly as difficult as deciding if $G$ has a planar spanning subgraph with minimum degree at least two.

We use a new set of gadgets; see Fig. 7.6. In addition to the gadgets that correspond to the components of the circuit network, we also have a gadget for each terminal. It can be verified that the distance between any pair of non-adjacent nodes within a gadget is larger than $\alpha$, and that there is an embedding that preserves this property.

As we are finding a subgraph of minimum degree 2, we change the definition of an internally good subgraph accordingly: each grey node must have degree at least 2 . With 


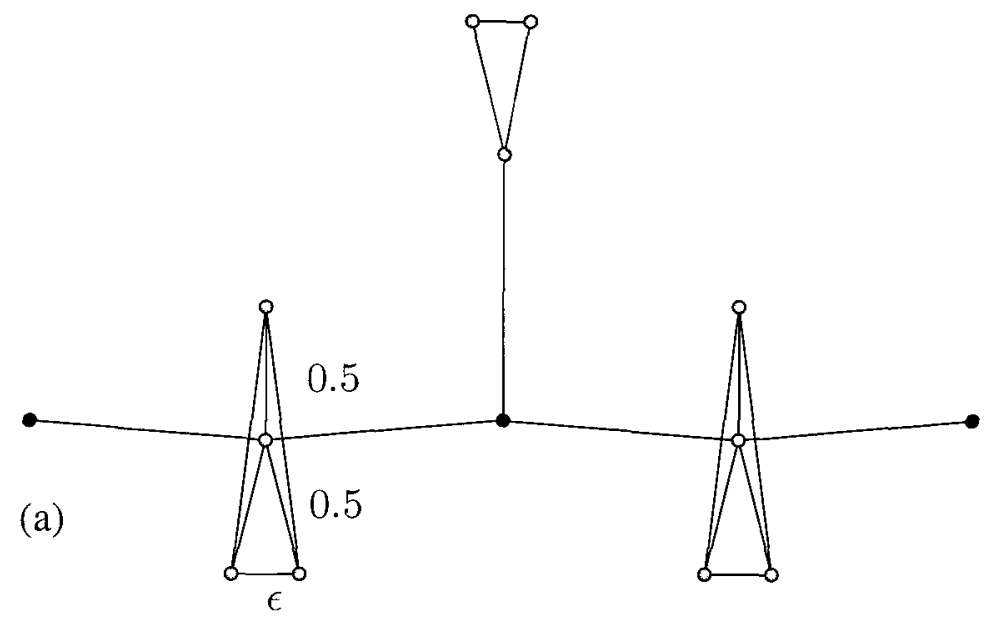

(b)
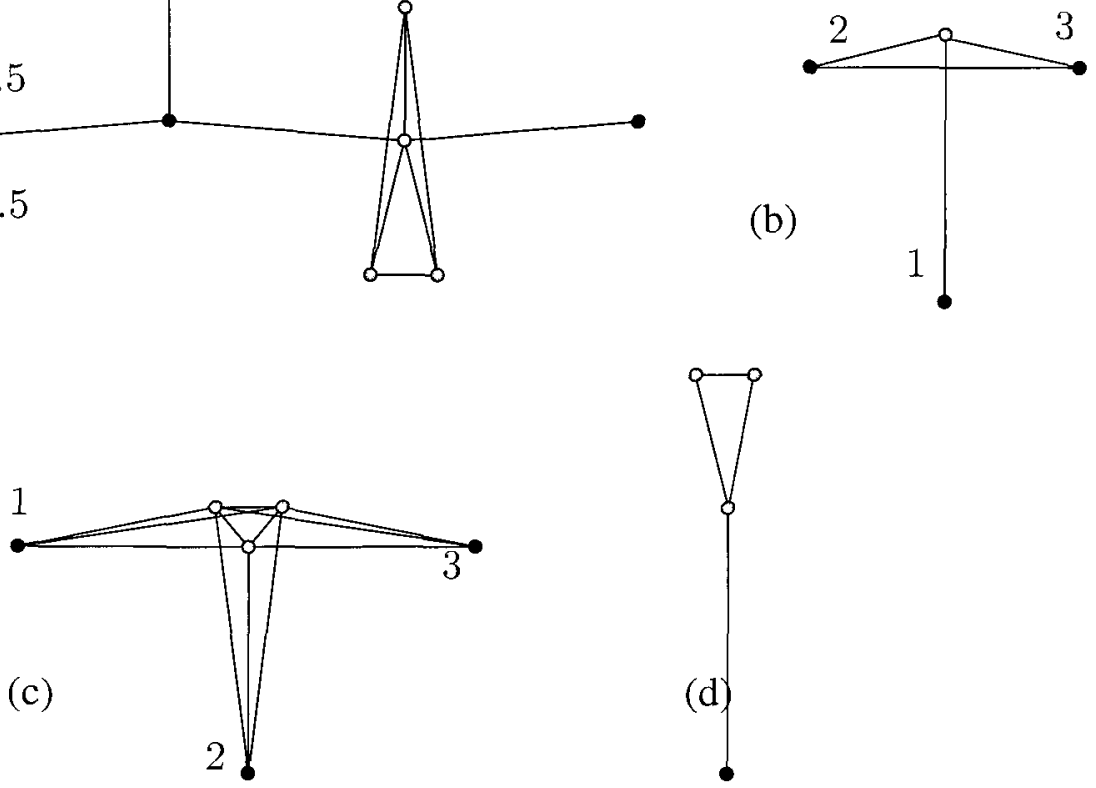

Figure 7.6: Gadgets for UDG, minimum degree 2: (a) wire, (b) switch, (c) user, (d) terminal.

this change, Lemma 43 holds verbatim.

The key difference with the proof of Theorem 42 is how we have to deal with the terminal gadgets. If we are given a valid orientation of $\mathcal{C}$, we can apply Lemma 43 to each component $\mathcal{C}[a]$ to find an internally good subgraph $G^{\prime}[a]$ for each $G[a]$. The union of the subgraphs $G^{\prime}[a]$ and all terminal gadgets forms a subgraph $G^{\prime}$ of $G$. By construction, $G^{\prime}$ is planar and each grey node has degree at least 2 . Now consider a terminal $t$. In a valid orientation, there is a component $\mathcal{C}[a]$ such that $a \rightarrow t$, and we have $d_{G^{\prime}[a]}(t) \geq 1$. Furthermore, $t$ is incident to exactly one edge from the terminal gadget. In summary, $d_{G^{\prime}}(t) \geq 2$. Hence $G^{\prime}$ is a planar spanning subgraph of $G$ and all nodes have degree at least 2

Conversely, given a planar spanning subgraph $G^{\prime}$ with minimum degree 2, we can verify that we can construct a valid orientation of $C$ : each terminal gadget contributes only one edge, and hence for each terminal $t$ we must have $a \rightarrow t$ such that $d_{G^{\prime}[a]}(t) \geq 1$. 
Observe that the embedding can be done in such a way that not adjacent vertices are at distance $\frac{\sqrt{5}}{2}-\varepsilon$, for an arbitrary small $\varepsilon>0$. This concludes the proof.

\subsection{Degree Three}

In this section, we consider the case of planar spanning subgraphs with minimum degree at least $k=3$. This case turns out to be similar to that of $k=2$ in Section 7.5. Again, we can prove that the problem is NP-complete, and even the augmentation version of the problem is NP-complete.

Theorem 46. The following promise problem is NP-complete for any $1 \leq \alpha<\sqrt{5} / 2$ : given a graph $G \in$ UDG, decide whether (i) there is no planar spanning subgraph of $G$ such that each node has degree at least 2, or (ii) there is a planar spanning subgraph of $G^{(\alpha)}$ such that each node has degree at least 2.

Proof. The proof is similar to Theorem 42. The key differences are as follows: We have a new set of gadgets, see Fig. 7.7. We do not have any terminal gadgets. The definition of an internally good subgraph is changed accordingly: each grey node has degree at least 3 . Finally, Lemma 43 is modified as follows.

Lemma 47. Given a valid orientation of $C[a]$, we can find an internally good subgraph $G^{\prime}[a]$ such that $a \bullet t$ implies $d_{G^{\prime}[a]}(t) \geq 1$ and $a \rightarrow \bullet$ implies $d_{G^{\prime}[a]}(t) \geq 2$. Conversely, given an internally good subgraph $G^{\prime}[a]$, we can find a valid orientation of $C[a]$ such that $a-\bullet t$ and $d_{G^{\prime}[a]}(t) \geq 2$ implies $a \multimap \bullet t$.

Now if we are given a valid orientation of $\mathcal{C}$, we can apply Lemma 47 to each component in order to construct a planar subgraph $G^{\prime}$ of $G$. For each terminal $t$ there are $a-\triangleright \bullet t$ and $b \bullet t$ with $a \neq b$. Now $d_{G^{\prime}}(t)=d_{G^{\prime}[a]}(t)+d_{G^{\prime}[b]}(t) \geq 2+1=3$. The converse case is similar.

\subsection{Conclusion}

In this chapter we have studied the problem of finding a planar spanning subgraph with minimum degree $k$ in different families of geometric graphs. One of the main discoveries is the existence of a very sharp threshold in the computational complexity of such problems: 

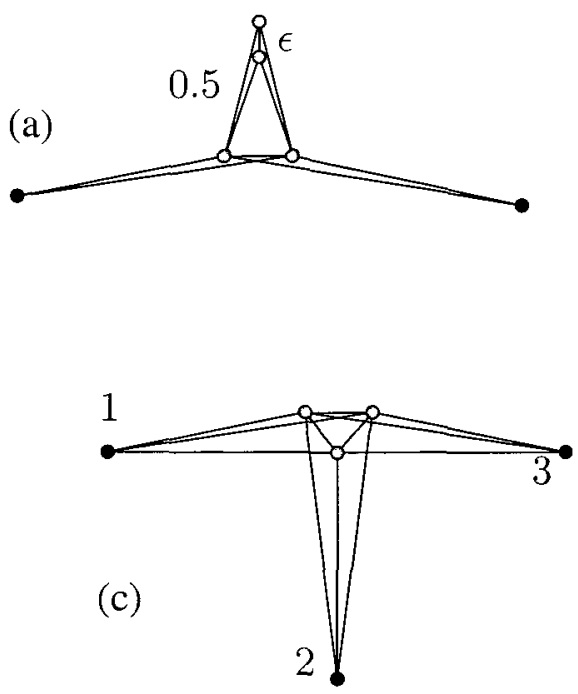

(b)

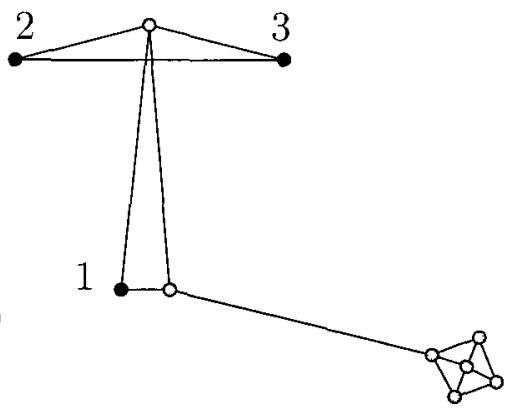

(d)

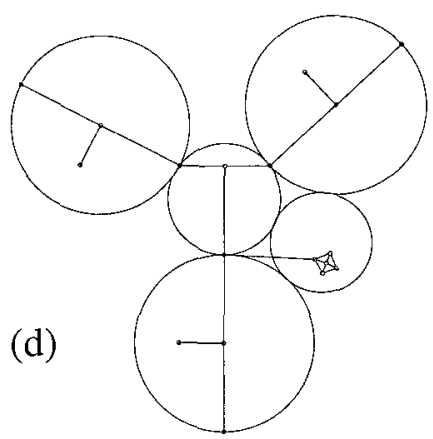

Figure 7.7: Gadgets for UDG and minimum degree 3: (a) wire, (b) switch, (c) user. (d) Embedding of a switch and three wires.

the case of $k=1$ and unit disk graphs is trivial, while a minor deviation from $k=1$ to $k=2$, or from unit disk graphs to quasi unit disk graphs makes the problem NP-Complete.

A major open problem is to determine for which values of the parameter $\alpha$ it can be solved in polynomial time. The next chapter is devoted to the study of this problem. 


\section{Chapter 8}

\section{Length 2-Times Optimal, 2-Edge Connected Planar Graph}

\subsection{Introduction}

Consider a set of points $P$ in the plane in general position. What is the minimum edge length necessary to create a $k$-edge and $k$-vertex connected planar spanning graph of $P$ with straight line edges? When $k=1$, the problem is optimally solved in polynomial time by taking the Euclidean MST on $P$ that minimizes the longest edge. In this chapter we study the case for 2-edge connectivity.

Formally, we can restate the question as follows: Given a set of point $P$ what is the minimum radius $r$ such that $\operatorname{UDG}(P, r)$ has an underlying two-edge connected planar graph? If $r$ is unbounded, then the problem is easy to solve. For example, the Delaunay Triangulation on $P$ is always two-edge connected and planar. On the other hand, an upper bound of 3 can be obtained using the results of Chapter 6 . However, this construction only relates the optimal radius to the radius necessary to create a connected graph. To close the gap we can relate the optimal radius with the radius necessary to create a two-edge connected graph on $P$.

A necessary condition to ensure two connectivity and planarity of a UDG is to decide whether it has a planar graph of minimum degree two. In Chapter 7 we have proved that given a $\operatorname{UDG}(P, 1)$ with minimum degree two, deciding whether $\operatorname{UDG}\left(P, \frac{\sqrt{5}}{2}-\varepsilon\right)$ has an underlying planar graph with minimum degree two is NP-Hard for any positive $\varepsilon$. Hence, $\frac{\sqrt{5}}{2}-\varepsilon$ turns out to be the lower bound for this problem. On the opposite direction, the optimal radius such that a UDG is either $k$-edge connected or $k$-vertex connected or has minimum degree $k$ can be computed in polynomial time. That is, we can test it in polynomial time for each of the possible $n(n-1) / 2$ distances.

Intuitively, one may consider to increase the connectivity of a given UDG to obtain a new graph that contains an underlying 2-edge connected planar subgraph. We will show that even for UDGs with high connectivity this is not always true. More specifically, we 
show that there exists $k$-vertex connected UDGs that do not have 2-edge connected planar spanning graph for any $k \in O(\sqrt{n})$. Further, a scaling factor of $17 / 16$ times the optimal radius is sometimes necessary.

\subsubsection{Our Results}

In Section 8.2 we present an algorithm that solves the augmentation problem in narrow strips of height $h$ in polynomial time for $k=2$ and scaling factor $\sqrt{1+h^{2}}$.

In Section 8.3, we give general concepts for the general proofs. Consider a set $P$ of $n$ point in the plane. Let $r_{1}$ be the minimum radius such that $\operatorname{UDG}\left(P, r_{1}\right)$ has minimum degree two, $r_{2}$ be the minimum radius such that $\operatorname{UDG}\left(P, r_{2}\right)$ is connected and has minimum degree two and $r_{3}$ be the minimum radius such that $\operatorname{UDG}\left(P, r_{3}\right)$ is two-edge connected.

In Section 8.4, we present an algorithm that obtains a planar subgraph with minimum degree two (not necessarily connected) from $\operatorname{UDG}\left(P, 2 \cdot r_{1}\right)$ that runs in $O(n \log n)$ time. In Section 8.5, we present an algorithm that obtains a 2-edge connected planar graph from $\operatorname{UDG}\left(P, \sqrt{5} \cdot r_{2}\right)$ that runs in $(n \log n)$ time. In section 8.6, we show that UDG $\left(P, 2 \cdot r_{3}\right)$ has a 2-edge connected planar graph and it can be obtained in $O\left(n^{2}\right)$ time. All the bounds on the length are tight. Further, in Section 8.7 we show that there exist $k$-vertex connected UDGs that do not have underlying 2-edge connected planar graphs even for a scaling factor of $17 / 16$ where $k \in O(\sqrt{n})$.

\subsection{Planar Graph of Points in a Narrow Strip with Bounded Length Edges}

As we saw in Section 7.5, the augmentation problem for the case of minimum degree $k=2$ is NP-complete in UDG if we are allowed to augment by adding edges of length $r<\sqrt{5} / 2$, and it can be solved in polynomial time if $r=3$. In this section we present an algorithm that solves the augmentation problem for $r=\sqrt{1+h^{2}}$ in narrow strips of height $h$.

Theorem 48. The following problem can be solved in polynomial time: given a UDG on a set $P$ of points inside a strip of height h, either

(i) show that there is no planar spanning subgraph of $\operatorname{UDG}(P, 1)$ such that each node has degree at least 2 , or

(ii) find a planar spanning subgraph of $\operatorname{UDG}(P, r)$ for $r=\sqrt{1+h^{2}}$ such that each node 
has degree at least 2.

Proof. Let $V=\left\{v_{1}, v_{2}, \ldots, v_{n}\right\}$ be the points of $P$ ordered by their $x$-coordinates, and let $x_{i}$ be the $x$-coordinate of node $v_{i}$; that is, we have $x_{i} \leq x_{i+1}$ for all $i<n$. We say that $V_{s, t}=\left\{v_{s}, v_{s+1}, \ldots, v_{t}\right\}$ is a section if

- $x_{i}+1 \geq x_{i+1}$ for all $s \leq i<t$,

- $s=1$ or $x_{s-1}+1<x_{s}$, and

- $t=n$ or $x_{t}+1<x_{t+1}$.

In other words, a section is a maximal set of nodes such that the $x$ coordinates are separated by at most 1 unit. Note that the subgraph $G_{s, t}$ induced by section $V_{s, t}$ consists of one or more connected components of $\operatorname{UDG}(P, 1)$.

Now consider each subgraph $G_{s, t}$ one by one; we will either conclude that we have case (i), or we will show how find a planar subgraph $G_{s, t}^{\prime}$ of $G_{s, t}^{(\alpha)}$ :

- If $t-s \leq 5$, we have a constant-size subproblem that we can solve by brute force: either decide that we have case (i), or construct a planar subgraph $G_{s, t}^{\prime}$.

- If $x_{s}+1<x_{s+2}$, then the degree of $v_{s}$ in $\operatorname{UDG}(P, 1)$ is at most one, and we have case (i). Similarly, if $x_{t-2}+1<x_{t}$, then the degree of $v_{t}$ in $\operatorname{UDG}(P, 1)$ is at most one, and we have case (i).

- Otherwise we can construct $G_{s, t}^{\prime}$ as follows: the edge set of $G_{s, t}^{\prime}$ consists of $\left\{v_{s}, v_{s+2}\right\}$, $\left\{v_{t-2}, v_{t}\right\}$, and $\left\{v_{i}, v_{i+1}\right\}$ for all $s \leq i<t$; each of these has length at most $r$, and they are non-intersecting.

\subsection{General Concepts}

Recall that $C(x ; r)$ denotes the circle centered at $x$ of radius $r$ and $D(x ; r)$ denotes the disk centered at $x$ of radius $r$. We introduce the concepts of Tie and Bow that will be useful in later proofs. Motivation will become apparent in the sequel. 
Definition 49. We say that four points $u, v, x, y$ form a Tie, denoted by Tie $(u ; v, x, y)$, if $\{u, v\}$ crosses $\{x, y\}, x$ and $y$ are outside $D(u ; d(u, v))$ and $u$ is outside $D(x ; d(x, y))$. The point $u$ is called tip of the Tie and $x y$ the crossing line of $\{u, v\}$.

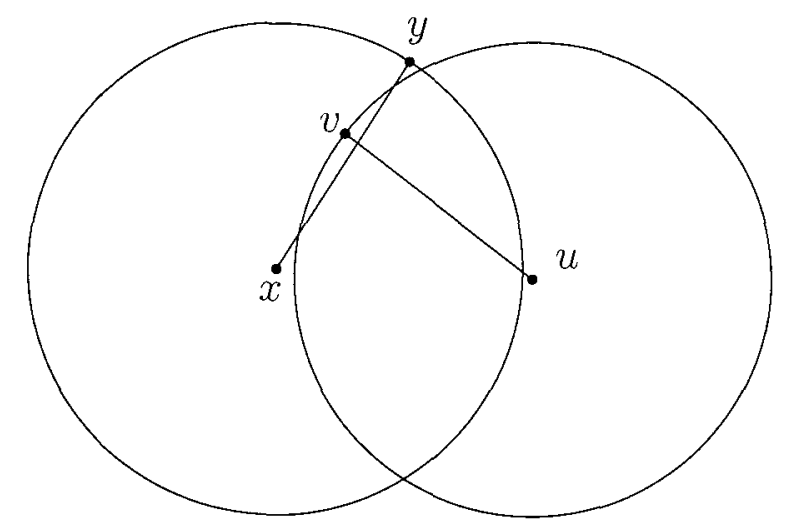

Figure 8.1: Tie $(u ; v, x, y)$ with tip $u$.

Lemma 50. Let $u, v, x, y$ form a Tie $(u ; v, x, y)$. Then, $\pi / 3 \leq \angle(u v x)<2 \pi / 3$ and $\pi / 3 \leq$ $\angle(y v u)<2 \pi / 3$.

Proof. Consider the angle $\angle(y v x)$, Observe that $\angle(y v x) \geq \pi / 2$ since from Definition 49 , $x, y \notin D(x ; d(x, y))$ and $u v$ crosses $x y$. Therefore, $d(x, y)>\max (d(x, v), d(v, y))$. Also from Definition $49, d(u, x)>d(x, y)$. Therefore, $\angle(u v x) \geq \pi / 3$ since it is the largest angle in the triangle $\triangle(u v x)$. It remains to prove that $\angle(y v u) \geq \pi / 3$ and the result follows since $\angle(y v x)<\pi$. For the sake of contradiction assume that $\angle(y v u)<\pi / 3$. From Definition 49 , $d(u, v)<d(u, y)$. Hence, $\angle(u y v)<\angle(y v u)$ and consequently $\angle(y u v)$ is the largest angle in $\triangle(u v y)$; see Figure 8.2. Therefore, $\angle(y u x)>\angle(y u v)>\angle(u y v)>\angle(u y x)$ which implies that $d(x, y)>d(u, x)$. This contradicts Definition 49 .

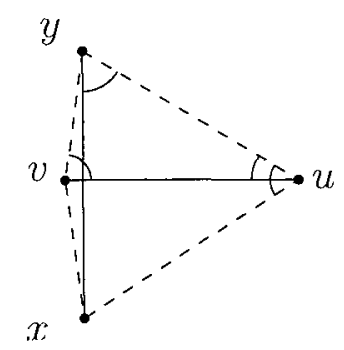

Figure 8.2: If $u, v, x, y$ form a $\operatorname{Tie}(u ; v, x, y)$ then $\angle(y v x) \geq 2 \pi / 3$. 
Lemma 51. Let $u, v, x, y$ form a $\operatorname{Tie}(u ; v, x, y)$ and $u^{\prime}$ be a point.

(i) If $\left\{u^{\prime}, v\right\}$ crosses $\{u, x\}$ then $u^{\prime}, v, u, x$ cannot form a Tie $\left(u^{\prime}, v, u, x\right)$.

(ii) If $\left\{u^{\prime}, x\right\}$ crosses $\{u, v\}$ then $u^{\prime}, x, u, v$ cannot form a $\operatorname{Tie}\left(u^{\prime}, x, u, v\right)$.

Proof. (i) Assume by contradiction that $u^{\prime} v$ and $u x$ form a Tie $\left(u^{\prime} ; v, u, x\right)$; see Figure 8.3a. From Lemma 50, $\angle(x v u) \geq 2 \pi / 3$. Now consider the Tie $(u ; v, x, y)$. From Lemma 50, $\angle(x v u)<2 \pi / 3$. A contradiction.

(ii) From Lemma 50, $\angle(u v x) \geq \pi / 3$. Therefore, $\angle(v x u)<2 \pi / 3$. However, the minimum angle $\angle(v x u)$ to form a $\operatorname{Tie}\left(u^{\prime} ; x, u, v\right)$ is at least $2 \pi / 3$; see Figure $8.3 \mathrm{~b}$.

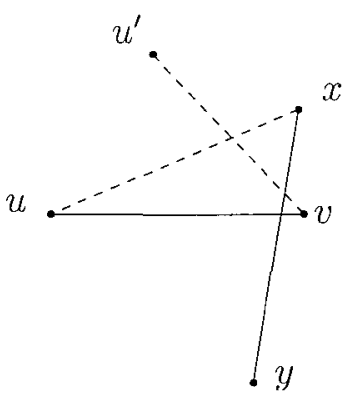

(a) $\left\{u^{\prime}, v\right\}$ and $\{u, x\}$ cannot form a Tie.

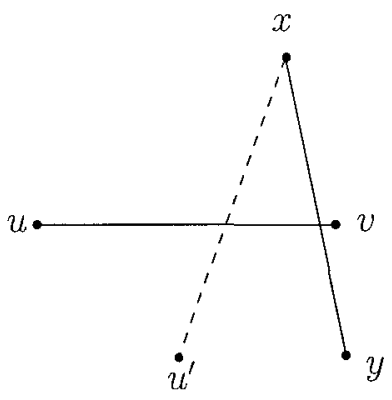

(b) $\left\{u^{\prime}, x\right\}$ and $\{u, v\}$ cannot form a Tie.

Figure 8.3: If $u, v, x, y$ form a Tie $(u ; v, x, y)$ then $u^{\prime}$ cannot form a Tie with either $v$ or $x$ or $y$ that overlaps Tie $(u ; v, x, y)$.

The following lemma shows that the points of a $\operatorname{Tie}(u ; v, x, y)$ are at distance at most $\sqrt{2}$ of each other.

Lemma 52. Let $u, v, x$ and $y$ be four points forming a $\operatorname{Tie}(u ; v, x, y)$ such that $\max (d(u, v)$, $d(x, y))=1$. Then, $d(u, x)$ and $d(u, y)$ are bounded by $\sqrt{2}$.

Proof. Let $p$ be the intersection point of $\{x, y\}$ and $C(u ; d(u, v))$ closer to $y$ and $l$ be the tangent line at $p$; see Figure 8.4. Since the angle that $\{u, p\}$ forms with $l$ is $\pi / 2$, $\angle(u p x) \leq \pi / 2$. Therefore, $d(u, x) \leq \sqrt{2}$ since $\max (d(u, p), d(p, x)) \leq 1$. Similarly, we can prove that $d(u, y) \leq \sqrt{2}$.

We conclude this section by introducing the concept of a Bow which will help to distinguish crossings in the proof of the main results. 


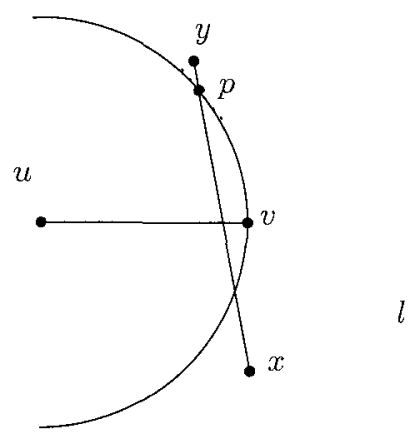

Figure 8.4: In a $\operatorname{Tie}(u ; v, x, y) d(u, x) \leq \sqrt{2}$ and $d(u, y) \leq \sqrt{2}$

Definition 53. We say that four points $u, v, x, y$ form a Bow denoted by $\operatorname{Bow}(u, v, x, y)$ if $\{u, v\}$ crosses $\{x, y\}, d(u, y) \leq d(u, v)<d(u, x)$ and $d(v, x) \leq d(x, y)<d(u, x)$.

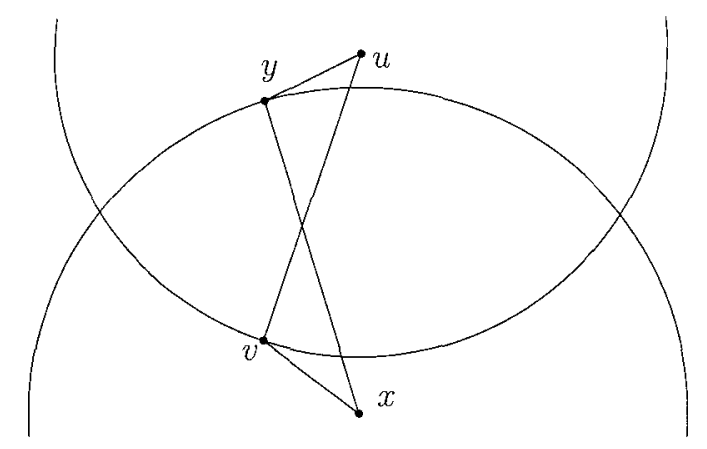

Figure 8.5: $\operatorname{Bow}(u, v, x, y)$.

\subsection{Planar Graph of UDGs with Minimum Degree 2}

In this section we prove that given a UDG with minimum degree two, a scaling of two is always sufficient and sometimes necessary to construct a planar graph with minimum degree two. Recall that $r_{1}$ is the minimum radius necessary to construct a UDG with minimum degree two. To simplify notation we assume that $r_{1}=1$.

\subsubsection{Lower Bound}

The following theorem shows that there exists a family of UDGs that requires edges of length 2 times the optimal. 
Theorem 54. There exist families of UDGs with $4 k$ vertices and minimum degree two that requires a scaling factor of 2 to construct a planar graph of degree two.

Proof. It is not difficult to see that the component depicted in Figure 8.6 requires $\{u, v\}$ to create a planar graph of degree two. To create a family of UDGs with $4 k$ vertices, it is enough to consider $k$ disconnected components.

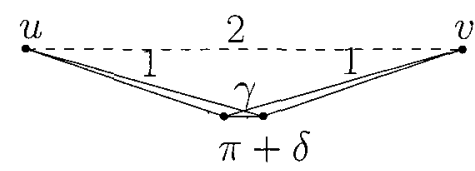

Figure 8.6: UDG of minimum degree two that requires scaling factor of 2.

\subsubsection{Upper Bound}

Let $P$ be a set of points and $T=(P, E)$ be an MST on $P$. Let $u$ be a leaf of $T$ and $v$ be the second nearest neighbor of $u$. (If there exist more than one then choose any arbitrary one among them.) The directed edge $(u, v)$ is defined as a Second Nearest Neighbor Edge or SNN edge for short. Let $E^{\prime}$ be the set of SNN edges. Observe that $E \cap E^{\prime}=\emptyset$ since the Nearest Neighborhood Graph is a subgraph of the MST.

Before giving the main theorem we provide some lemmas that are required for the proof. The following lemma shows that a SNN edge $(x, y)$ that crosses an edge $\{u, v\}$ of the MST forms a Tie $(u ; v, x, y)$.

Lemma 55. Let $T$ be an MST and $(x, y)$ be a SNN edge that crosses an edge $\{u, v\} \in T$. Then, they form a Tie $(u ; v, x, y)$ such that either $\{u, x\} \in T$ or $\{v, x\} \in T$. Moreover, the quadrangle uxvy is empty.

Proof. First we will show that if $(x, y)$ crosses $\{u, v\}$ then either $\{u, x\} \in T$ or $\{v, x\} \in T$. For the sake of contradiction assume that neither $\{u, x\} \notin T$ nor $\{v, x\} \notin T$. Observe that $u$ and $v$ are outside $D(x ; d(x, y))$, otherwise $(x, y)$ would not be the SNN edge; see Figure $8.7 \mathrm{a}$. Therefore, $\angle(v y u) \geq \pi / 2$ since $(x, y)$ crosses $\{u, v\}$. Hence, $d(u, v)$ is greater than $d(u, y)$ and $d(v, y)$. This contradicts the assumption since a spanning tree of less weight can be constructed by replacing $\{u, v\}$ with either $\{u, y\}$ or $\{v, y\}$. 
To show that they form a $\operatorname{Tie}(u ; v, x, y)$, assume that $\{v, x\} \in T$. Observe that $d(u, x)>$ $d(x, y)>\max (d(v, x), d(v, y))$ since $y$ is the second nearest neighbor of $x$ and $\angle(x v y) \geq \pi / 2$; see Figure 8.7b. It is not difficult to see that $d(u, v)<d(u, x)$ (Otherwise we can obtain a better MST by replacing $\{u, v\}$ with $\{u, x\}$.) To prove that $d(u, v)<d(u, y)$ assume by contradiction that $d(u, v)>d(u, y)$. Hence, $\angle(y u v)$ is the largest angle in $\triangle(u v y)$ since $d(u, v)<d(v, y)$ (Otherwise we can obtain a better MST by replacing $\{u, v\}$ with either $\{u, y\}$ or $\{v, y\}$.) Therefore, $\angle(y u x)>\angle(x y u)$ which implies that $d(x, y)>d(x, u)$. This is a contradiction since $d(u, x)<d(x, y)$.

To prove that $u x v y$ is empty, we consider independently $\triangle(u v x)$ and $\triangle(u v y)$. First consider $\triangle(u v x)$. It is known that the angle that a vertex forms with two consecutive neighbors in the MST is at least $\pi / 3$ and the triangle is empty. Therefore, $v$ does not have a neighbor in the sector $\angle(x v u)$ since by Lemma $50 \angle(u v x)<2 \pi / 3$. Therefore, $\triangle(u v x)$ is empty. Now we consider $\triangle(u v y)$. Assume by contradiction that exists a point $p$ in $\triangle(u v y)$ as depicted in Figure 8.7c. Observe that $\angle(u v p)>\pi / 3$ (Otherwise we can replace $\{u, v\}$ with either $\{u, p\}$ or $\{v, p\}$.) Therefore, $\angle(x v p)<\angle(x v y)$ and $d(x, p)<d(x, y)$ since $d(v, p) \leq d(v, y)$ which contradicts the SNN edge definition.

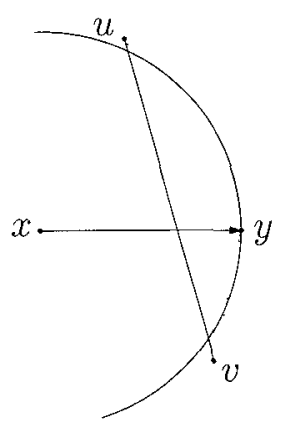

(a) $\{u, v\} \notin$ $D(x ; d(x, y))$

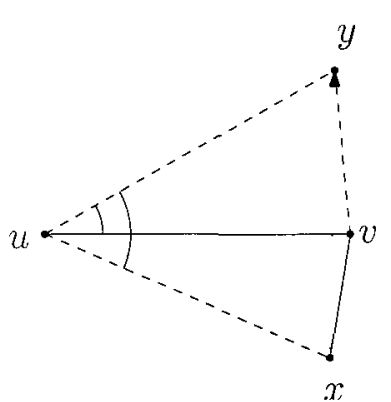

(b) A SNN edge that crosses an MST edge forms a Tie.

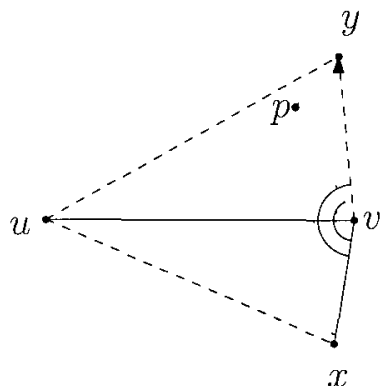

(c) $u x v y$ is empty.

Figure 8.7: A SNN crossing an edge of the MST.

As a consequence of Lemma 55, a SNN edge crosses at most one edge of the MST since the angle that a vertex forms with two consecutive neighbors in an MST is at least $\pi / 3$. The following lemma will help to characterize crossings between SNN edges. 
Lemma 56. Let $(u, v)$ and $\left(u^{\prime}, v^{\prime}\right)$ be two crossing $S N N$ edges. Then either $\left\{u^{\prime}, v\right\} \in T$ or $\left\{u, v^{\prime}\right\} \in T$.

Proof. Assume that $\left\{u^{\prime}, v\right\},\left\{u, v^{\prime}\right\} \notin T$, then $u^{\prime}$ and $v^{\prime}$ are not in $D(u ; d(u, v))$ as depicted in Figure 8.8. Observe that if either $u^{\prime}$ or $v^{\prime}$ is in $D(u ; d(u, v))$, then $(u, v)$ would not be the SNN edge. Therefore, $d\left(u^{\prime}, v^{\prime}\right)>\max \left(d\left(u^{\prime}, v\right), d\left(v, v^{\prime}\right)\right)$ since $\angle\left(v^{\prime} v u^{\prime}\right)>\pi / 2$ and $(u, v)$ crosses $\left(u^{\prime}, v^{\prime}\right)$. This is a contradiction since $\left\{u^{\prime}, v\right\} \notin T$.

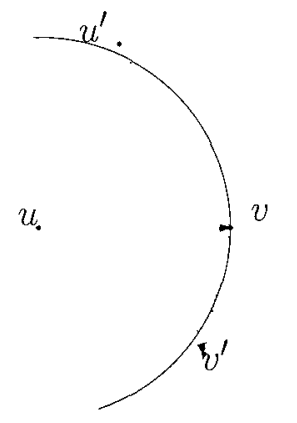

Figure 8.8: Two SNN edges crossing.

Lemma 57. Let $(u, v)$ and $\left(u^{\prime}, v^{\prime}\right)$ be two crossing SNN edges.

(i) If $\left\{\left\{u, v^{\prime}\right\},\left\{u^{\prime}, v\right\}\right\} \in T$, then they form a $\operatorname{Bow}\left(u, v, u^{\prime}, v^{\prime}\right)$ such that the quadrangle $u v^{\prime} v u^{\prime}$ is empty.

(ii) If $\left\{u^{\prime}, v\right\} \in T$ and $\left\{u, v^{\prime}\right\} \notin T$, then they form a Tie $\left(u ; v, u^{\prime}, v^{\prime}\right)$ such that the quadrangle $u u^{\prime} v v^{\prime}$ either is empty or contains the neighbor of $u$ in $T$.

Proof. (i) Let $\left\{u, v^{\prime}\right\} \in T$ and $\left\{u^{\prime}, v\right\} \in T$. Clearly, $d\left(u, u^{\prime}\right)>d(u, v)>d\left(u, v^{\prime}\right)$ since $v^{\prime}$ is the nearest neighbor of $u$ and $v$ the second. Similarly, $d\left(u, u^{\prime}\right)>d\left(u^{\prime}, v^{\prime}\right)>d\left(u^{\prime}, v\right)$. Therefore, they form a $\operatorname{Bow}\left(u, v, u^{\prime}, v^{\prime}\right)$. To prove that the quadrangle $u v^{\prime} v u^{\prime}$ is empty consider $R=D(u ; d(u, v)) \cup D\left(u^{\prime} ; d\left(u^{\prime}, v^{\prime}\right)\right)$ as depicted in Figure 8.9a. Obviously any point inside $R$ is closer to either $u$ or $u^{\prime}$. Therefore, $R$ contains only $u, v, u^{\prime}, v^{\prime}$.

(ii) Let $\left\{u^{\prime}, v\right\} \in T$ and $\left\{u, v^{\prime}\right\} \notin T$. From the definition of SNN edge $d(u, v) \leq$ $\min \left(d\left(u, u^{\prime}\right), d\left(u, v^{\prime}\right)\right)$ and $d\left(u^{\prime}, v^{\prime}\right)<d\left(u, u^{\prime}\right)$. Therefore, they form a Tie $\left(u ; v, u^{\prime}, v^{\prime}\right)$. To prove that the quadrangle may contain at most one point $p$ such that $\{u, p\} \in T$ consider $R=D(u ; d(u, v)) \cup D\left(u^{\prime} ; d\left(u^{\prime}, v^{\prime}\right)\right)$ as depicted in Figure 8.9b. Obviously any point inside $R$ is closer to either $u$ or $u^{\prime}$. Therefore, it contains only the nearest neighbors of $u$ and $u^{\prime}$. 
Further, $v$ is the nearest neighbor of $u^{\prime}$. Therefore, $p \in R$ where $\{u, p\} \in T$. It remains to prove that $R$ contains the quadrangle $u u^{\prime} v v^{\prime}$. Let $a$ be the intersection point of $\left\{u, v^{\prime}\right\}$ and $C(u ; d(u, v))$. It is enough to prove that $a \in D\left(u^{\prime} ; d\left(u^{\prime}, v^{\prime}\right)\right)$. However, $\angle\left(u^{\prime} v a\right)<\angle\left(u^{\prime} v v^{\prime}\right)$ and $d(v, a) \leq d\left(v, v^{\prime}\right)$. Therefore, $d\left(u^{\prime}, a\right)<d\left(u^{\prime}, v^{\prime}\right)$.

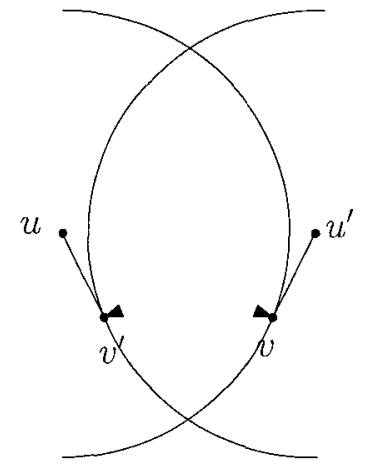

(a) $\left\{\left\{u, v^{\prime}\right\},\left\{u^{\prime}, v\right\}\right\} \in$
$T$

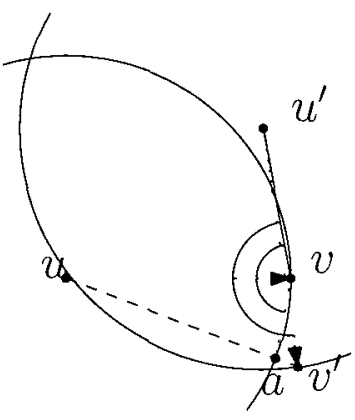

(b) $\left\{u^{\prime}, v\right\} \in T$ and $\left\{u, v^{\prime}\right\} \notin T$

Figure 8.9: Crossings of SNN edges.

The following lemma will help to bound the length of the new edges.

Lemma 58. Let $u, v, u^{\prime}, v^{\prime}$ be four vertices forming a Tie $\left(u ; v, u^{\prime}, v^{\prime}\right)$ and $w$ be a vertex such that $d(u, w) \leq 1, \angle(w u v) \leq \varphi$ and $\left\{u^{\prime}, u\right\}$ crosses $\{w, v\}$. Then, $d\left(w, u^{\prime}\right)^{2} \leq 3-$ $2 \sqrt{2} \cos (\varphi-\pi / 4)$.

Proof. Observe that $\left\{u^{\prime}, v^{\prime}\right\}$ crosses two points of $C(u ; d(u, v))$. Thus, we can assume without loss of generality that $\left\{u^{\prime}, v^{\prime}\right\}$ crosses $C(u ; d(u, v))$ in $v$ and $d(u, v)=d\left(u, v^{\prime}\right)$ as depicted in Figure 8.10. Let $\alpha=\angle\left(v u v^{\prime}\right)$ and $\beta=\angle\left(u v^{\prime} v\right)=\angle\left(v^{\prime} v u\right)=\frac{\pi-\alpha}{2}$. Observe that $0<\alpha \leq \pi / 3$ since from Lemma $50, \angle\left(u \nu v^{\prime}\right) \geq \pi / 3$. From the law of cosine in $\triangle\left(u v^{\prime} u^{\prime}\right)$, $d\left(u, u^{\prime}\right)^{2}=d\left(u, v^{\prime}\right)^{2}+d\left(u^{\prime}, v^{\prime}\right)^{2}-2 d\left(u, v^{\prime}\right) d\left(u^{\prime}, v^{\prime}\right) \cos (\beta) \leq 2-2 \cos (\beta)=2-2 \sin (\alpha / 2)$ and $d\left(u, u^{\prime}\right) \leq 2 \sin \left(\frac{\beta}{2}\right)=2 \cos \left(\frac{\pi-\alpha}{4}\right)$.

Let $\gamma=\angle\left(w u u^{\prime}\right)=\varphi-\angle\left(u^{\prime} u v\right)$. Since $\angle\left(v^{\prime} v u\right)=\beta, \angle\left(u v u^{\prime}\right)=\pi-\beta$. Therefore, if $d(u, v) \leq d\left(u^{\prime}, v\right)$, then $\angle\left(u^{\prime} u v\right) \geq \frac{\pi-(\pi-\beta)}{2}=\frac{\pi-\alpha}{4}$. Otherwise, $\angle\left(v u^{\prime} u\right) \geq \frac{\pi-(\pi-\beta)}{2}=\frac{\beta}{2}$. From $\triangle\left(u v^{\prime} u^{\prime}\right), \angle\left(u^{\prime} u v\right) \geq \pi-\beta-\frac{\beta}{2}-\alpha=\frac{\pi-\alpha}{4}$.

From the law of cosine, $d\left(w, u^{\prime}\right)^{2}=d(u, w)^{2}+d\left(u, u^{\prime}\right)^{2}-2 d\left(u, u^{\prime}\right) d(u, w) \cos (\gamma) \leq 3-$ $2 \sin \left(\frac{\alpha}{2}\right)-4 \cos \left(\frac{\pi-\alpha}{4}\right) \cos \left(\varphi-\frac{\pi-\alpha}{4}\right)$. Observe that when $0 \leq \alpha \leq \pi / 3$ and $\pi / 3 \leq \varphi \leq \pi$, the 
three values $\sin \left(\frac{\alpha}{2}\right), \cos \left(\frac{\pi-\alpha}{4}\right)$ and $\cos \left(\varphi-\frac{\pi-\alpha}{4}\right)$ are positive values. Therefore, for any $\varphi \in$ $[\pi / 3, \pi]$ the maximum value is reached when $\alpha=0$ and $d\left(w, u^{\prime}\right)^{2} \leq 3-2 \sqrt{2} \cos \left(\varphi-\frac{\pi}{4}\right)$.

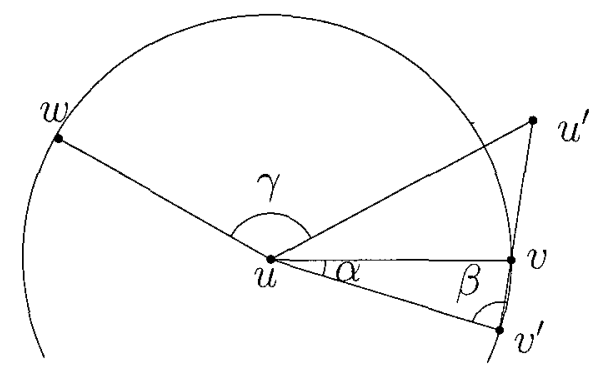

Figure 8.10: If $\angle(w u v) \leq \varphi$ then $d\left(w, u^{\prime}\right)^{2}<3-2 \sqrt{2} \cos \left(\varphi-\frac{\pi}{4}\right)$.

Now we are ready to give the main theorem.

Theorem 59. Let $P$ be a set of $n$ points in the plane such that $\operatorname{UDG}(P, 1)$ has minimum degree two. Then, $\operatorname{UDG}(P, 2)$ has a planar graph with minimum degree 2. Further, it can be constructed in time $O(n \log n)$.

Proof. Consider the Nearest Neighbor (undirected) Graph $T=(P, E)$. It is known that any MST on $P$ contains the edges of $T$. Hence, $T$ is a subgraph of the MST. Let $E^{\prime}$ be the set of SNN edges from leaves of $T$. Clearly every edge in $E^{\prime}$ has length at most one since $\operatorname{UDG}(P, 1)$ has minimum degree two. Let $G=\left(P, E \cup E^{\prime}\right)$.

Claim 2. Let Tie $\left(u ; v, u^{\prime}, v^{\prime}\right)$ be a Tie of $G$ where $u^{\prime}$ is a leaf of $T$.

(i) $\{u, v\}$ may cross at most another edge $\left\{u^{\prime \prime}, v^{\prime \prime}\right\}$ such that they form either a Tie $\left(v ; u, u^{\prime \prime}, v^{\prime \prime}\right)$ or a $\operatorname{Tie}\left(u^{\prime \prime} ; v^{\prime \prime}, u, v\right)$.

(ii) $\left\{u^{\prime}, v\right\} \in E$ does not cross any edge.

Proof. (i) From Lemmas 55 and $56,\left\{u^{\prime}, v\right\} \in E$. Therefore, $v$ is not a leaf in $T$. Hence, if $u$ is a leaf of $T$, then from Lemma $56,\{u, v\}$ may be only the crossing line of a Tie $\left(u^{\prime \prime} ; v^{\prime \prime}, u, v\right)$ as depicted in Figure 8.11a. On the other hand, from Lemmas 55 if $u$ is not a leaf of $T$ then $v$ may be the tip of another Tie $\left(v ; u, u^{\prime \prime}, v^{\prime \prime}\right)$ as depicted in Figure 8.11b.

(ii) Assume by contradiction that $\left\{u^{\prime}, v\right\}$ crosses a SNN edge $(x, y)$ where $x$ is a leaf of $T$. Therefore, from Lemma 55 they form a Tie $\left(u^{\prime} ; v, x, y\right)$ where $\{x, v\} \in E$ since $u^{\prime}$ is a leaf. Observe that $(x, y)$ also crosses $\left(u^{\prime}, v^{\prime}\right)$ otherwise $\left(u^{\prime}, v^{\prime}\right)$ would not be the SNN edge. 
Therefore, from Lemma 56 either $\{v, x\} \in E$ or $\left\{u^{\prime}, y\right\} \in E$. This is a contradiction since $u^{\prime}$ and $x$ are leaves of $T$.
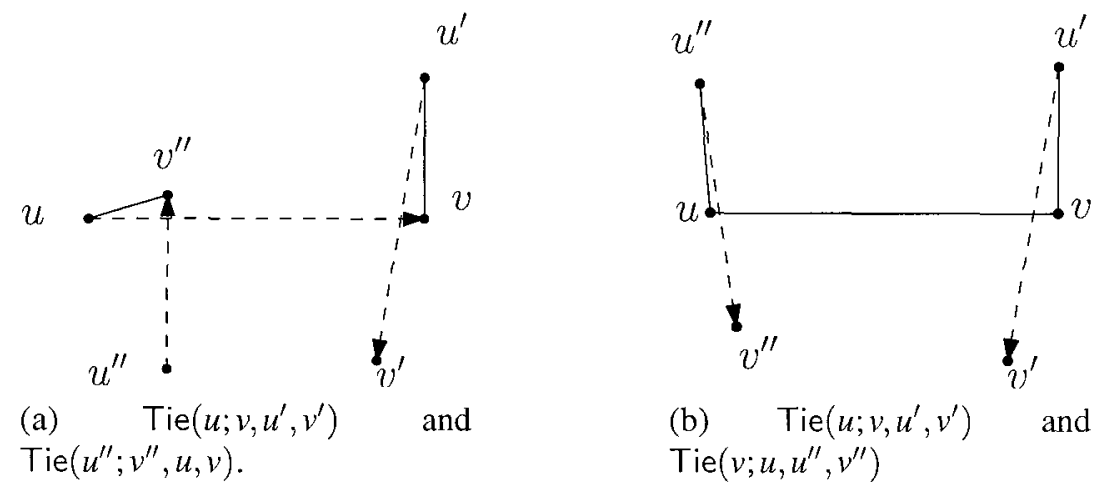

Figure 8.11: $\{u, v\}$ is in at most two Ties. (Solid lines are edges of $T$ and dashed arrow lines are SNN edges.)

The proof is constructive. In every step we remove at least one crossing of $G$ by replacing edges of $E^{\prime}$. First, we remove all the Ties and next the Bows.

Let Tie $\left(u ; v, u^{\prime}, v^{\prime}\right)$ be a Tie of $G$ where $u^{\prime}$ is a leaf of $T$. Observe that from Lemma 51 , there does not exist a leaf $r$ of $T$ such that either $(r, v)$ crosses $\left\{u^{\prime}, v^{\prime}\right\}$ or $\left(r, v^{\prime}\right)$ crosses $\{u, v\}$. According to Claim 2, three cases can occur:

(i) $\{u, v\}$ does not form another Tie. From Lemmas 55 and 56, $\triangle\left(u v u^{\prime}\right)$ is either empty or it has exactly one vertex $w$ such that $\{w, u\} \in E$. If $\triangle\left(u v u^{\prime}\right)$ is empty, let $E^{\prime}=E^{\prime} \cup\left\{\left\{u, u^{\prime}\right\}\right\} \backslash\left\{\left\{u^{\prime}, v^{\prime}\right\}\right\}$. Otherwise, let $E^{\prime}=E^{\prime} \cup\left\{\left\{w, u^{\prime}\right\}\right\} \backslash\left\{\left\{u^{\prime}, v^{\prime}\right)\right\}$; see Figure 8.12. From Lemma 52, $d\left(u, u^{\prime}\right) \leq \sqrt{2}$. Therefore the new edge is bounded by $\sqrt{2}$. Since $\{u, v\}$ and $\left\{v, u^{\prime}\right\}$ are not crossed, the new edge does not cross any edge of $G$.

(ii) $\{u, v\}$ forms a Tie $\left(v ; u, u^{\prime \prime}, v^{\prime \prime}\right)$ where $u^{\prime \prime}$ is a leaf of $T$. Observe that in this case $u$ and $v$ are not leaves of $T$. Therefore, from Lemma 55 the quadrangles $u u^{\prime} v v^{\prime}$ and $v u^{\prime \prime} u v^{\prime \prime}$ are empty. We consider two cases. In the first case $\left\{u, u^{\prime}\right\}$ does not cross $\left\{u^{\prime \prime}, v\right\}$. Let, $E^{\prime}=E^{\prime} \cup\left\{\left\{u, u^{\prime}\right\},\left\{u^{\prime \prime}, v\right\}\right\} \backslash\left\{\left\{u^{\prime}, v^{\prime}\right\},\left\{u^{\prime \prime}, v^{\prime \prime}\right\}\right\}$ as depicted in Figure $8.13 \mathrm{a}$. From Lemma 52 , the new edges are bounded by $\sqrt{2}$. In the second case $\left\{u, u^{\prime}\right\}$ crosses $\left\{u^{\prime \prime}, v\right\}$; see Figure $8.13 \mathrm{~b}$. Consider the quadrangle $u v u^{\prime} u^{\prime \prime}$. If 


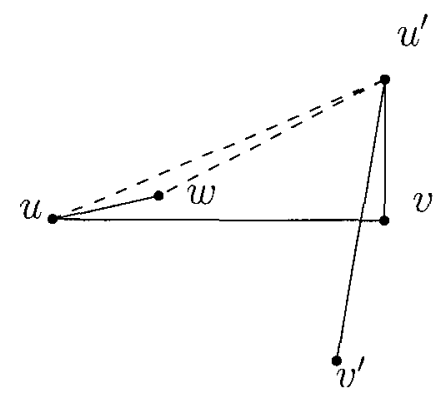

Figure 8.12: $\{u, v\}$ is in one Tie. (Dotted lines are removed edges and dashed lines are possible new edges.)

it is empty, let $E^{\prime}=E^{\prime} \cup\left\{\left\{u^{\prime}, u^{\prime \prime}\right\}\right\} \backslash\left\{\left\{u^{\prime}, v^{\prime}\right\},\left\{u^{\prime \prime}, v^{\prime \prime}\right\}\right\}$. Otherwise, let $p$ and $q$ be the vertices in $u v u^{\prime} u^{\prime \prime}$ such that $\angle\left(u u^{\prime \prime} p\right)$ and $\angle\left(v u^{\prime} q\right)$ are minimum. Let $E^{\prime}=$ $E^{\prime} \cup\left\{\left\{u^{\prime}, q\right\},\left\{u^{\prime \prime}, q\right\}\right\} \backslash\left\{\left\{u^{\prime}, v^{\prime}\right\},\left\{u^{\prime \prime}, v^{\prime \prime}\right\}\right\}$. From Lemma 58,d(u', $\left.u^{\prime \prime}\right) \leq 2$ since $\angle\left(u^{\prime \prime} u v\right) \leq 2 \pi / 3$. Observe that $p$ does not have a neighbor in the same half-space determined by $\left\{u^{\prime \prime}, p\right\}$ as $u$ because $\angle\left(u u^{\prime \prime} p\right)$ is minimum. Similarly, $q$ does not have a neighbor in the same half-space determined by $\left\{u^{\prime}, q\right\}$ as $v$ because $\angle\left(v u^{\prime} q\right)$ is minimum. Since, $\left\{v, u^{\prime}\right\}$ and $\left\{u, u^{\prime \prime}\right\}$ do not cross any other edge and $\{u, v\}$ only forms $\operatorname{Tie}\left(u ; v, u^{\prime}, v^{\prime}\right)$ and $\operatorname{Tie}\left(v ; u, u^{\prime \prime}, v^{\prime \prime}\right)$, the new edges do not cross any edge of $G$.

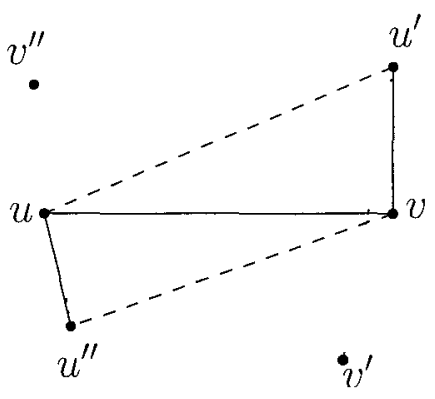

(a) $\{u, v\}$ is in one Tie.

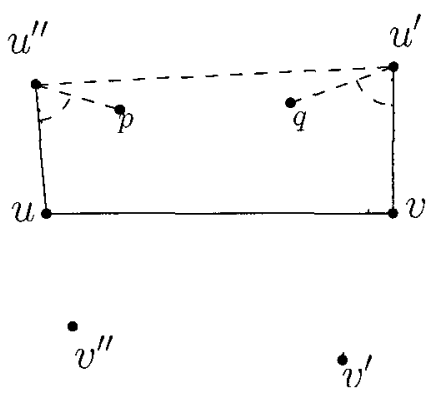

(b) $\{u, v\}$ is in two Ties

Figure 8.13: $\{u, v\}$ crosses at least one edge of $G$. (Dotted lines are removed edges and dashed lines are possible new edges.)

(iii) $\{u, v\}$ forms a $\operatorname{Tie}\left(u^{\prime \prime} ; v^{\prime \prime}, u, v\right)$. Observe that in this case $u$ is a leaf of $T$. Assume without loss of generality that $\left\{u^{\prime \prime}, v\right\}$ crosses $\left\{u, u^{\prime}\right\}$. Consider the quadrangle $u^{\prime \prime} u v u^{\prime}$. If it is empty, then let $E^{\prime}=E^{\prime} \cup\left\{\left\{u^{\prime}, u^{\prime \prime}\right\}\right\} \backslash\left\{\left\{u^{\prime}, v^{\prime}\right\}\right\}$. Otherwise, let $p$ be the vertex in $u^{\prime \prime} u v u^{\prime}$ such that $\angle\left(v u^{\prime} p\right)$ is minimum. Let $E^{\prime}=E^{\prime} \cup\left\{\left\{u^{\prime}, p\right\}\right\} \backslash$ $\left\{\left\{u^{\prime}, v^{\prime}\right\}\right\}$. From Lemma $58, d\left(u^{\prime}, u^{\prime \prime}\right) \leq 2$ since $\angle\left(u^{\prime \prime} u v\right) \leq 2 \pi / 3$. Observe that all 
the neighbors of $p$ are in the same half-plane determined by $\left\{u^{\prime}, p\right\}$. It is not difficult to see that the new edge does not cross any edge of $G$ since the region $u^{\prime \prime} u v u^{\prime}$ is close.

After removing the Ties we remove the Bows. Consider a Bow $\left(u, v, u^{\prime}, v^{\prime}\right)$ where $u$ and $u^{\prime}$ are leaves of $T$. Let $E^{\prime}=E^{\prime} \cup\left\{\left\{u, u^{\prime \prime}\right\}\right\} \backslash\left\{\{u, v\},\left\{u^{\prime}, v^{\prime}\right\}\right\}$. Clearly, $d\left(u, u^{\prime}\right) \leq 2$ and $\left\{u, u^{\prime \prime}\right\}$ does not cross any edge of $G$.

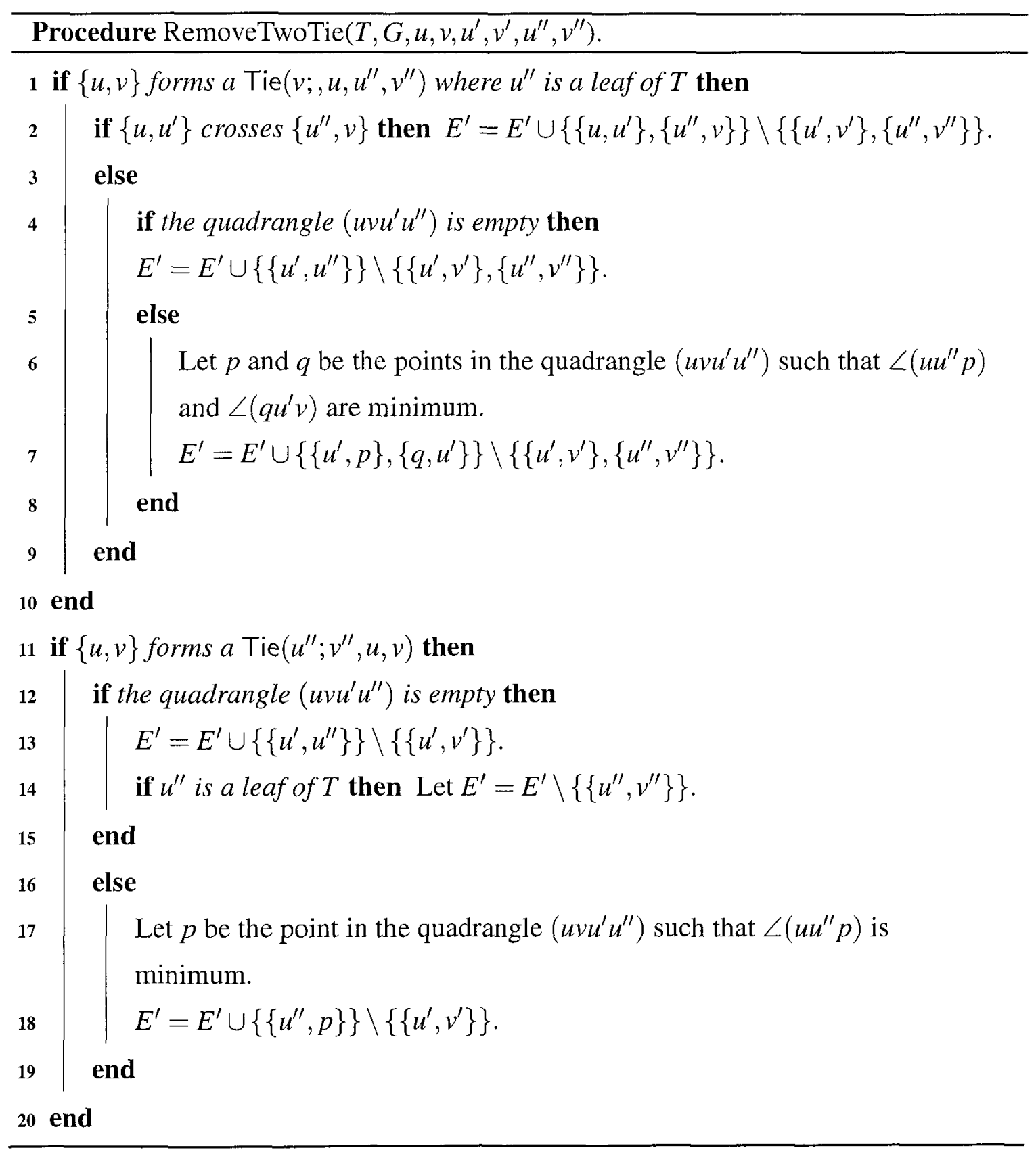


Algorithm 5: Constructing a Planar Graph with minimum degree two and longest edge length bounded by 2 .

input : UDG with minimum degree 2.

output: $G\left(V, E \cup E^{\prime}\right)$ : Planar Graph with minimum degree 2 and longest edge length bounded by 2 .

1 Let $T=(P, E)$ be the MST of the UDG.

2 Assign a two coloration to the vertices of $T$ with colors red and black.

3 Let $E^{\prime}$ be the set of SNN directed edges from black leaves.

4 Let $G=\left(P, E \cup E^{\prime}\right)$.

5 foreach edge $\{u, v\}$ in $G$ that forms a Tie $\left(u ; v, u^{\prime}, v^{\prime}\right)$ do

$6 \quad$ if $\{u, v\}$ does not form another Tie then

$7 \quad$ if $\triangle\left(u v u^{\prime}\right)$ is empty then $E^{\prime}=E^{\prime} \cup\left\{\left\{u, u^{\prime}\right\}\right\} \backslash\left\{\left\{u^{\prime}, v^{\prime}\right\}\right\}$.

$8 \quad$ else

9

10

11

16 end

17 foreach edge $\{u, v\}$ in $G$ that forms a $\operatorname{Bow}\left(u, v, u^{\prime}, v^{\prime}\right)$ do

$18 \mid E^{\prime}=E^{\prime} \cup\left\{u, u^{\prime}\right\} \backslash\left\{\{u, v\},\left\{u^{\prime}, v^{\prime}\right\}\right\}$

19 end

The pseudocode is presented in Algorithm 5. Regarding the complexity, the Nearest Neighbor Graph on the set of points can be constructed in $O(n \log n)$. A trapezoidal map can be also constructed in $O(n \log n)$ where a point localization takes $O(\log n)$ time [6]. The removal of a crossing can be done in time $O(\log n)$ and there exist at most $2 n$ Ties since each leaf of $T$ can form at most two Ties. Therefore, the whole construction can be done in $O(n \log n)$ since there are at most $O(n)$ crossings. This complete the proof. 


\subsection{2-Edge Connected Planar Graph of UDGs with Minimum Degree 2}

Recall that $r_{2}$ is the minimum radius necessary to construct a connected UDG with minimum degree two. To simplify notation we assume that $r_{2}=1$.

\subsubsection{Lower Bound}

In this subsection we show that there exist connected UDGs with minimum degree two that require edges of length bounded by $\sqrt{5}$ to create a 2-edge connected planar graph.

Theorem 60. For any $n \geq 8$ there exist families of connected UDGs with minimum degree two that require a scaling factor of $\sqrt{5}$ to construct a 2-edge connected planar graph.

Proof. Consider the component $C$ depicted in Figure 8.14. $x$ is called the entry point and has the following properties: $d(x)=1, d(v, x)>\sqrt{5}$ and $\left\{u_{2}, x\right\}$ crosses $C$. Observe that $C$ requires at least one of the edges $\left\{u_{1}, w\right\},\left\{u_{2}, v\right\}$ to be included so that the edge $\{v, w\}$ is in a 2-edge connected planar spanning graph. We may assume without loss of generality that the edge $\left\{u_{1}, w\right\}$ is added. Observe, that for any arbitrarily small $\varepsilon>0$, there exists $\delta>0$ sufficiently close to zero such that $d\left(u_{1}, w\right)$ is close to $\sqrt{5}$. Observe that $C \backslash x$ has minimum degree two. We can construct a family of UDGs with $n>8$ vertices and minimum degree two having the same lower bound by connecting the entry point $x$ to distinct UDG components.

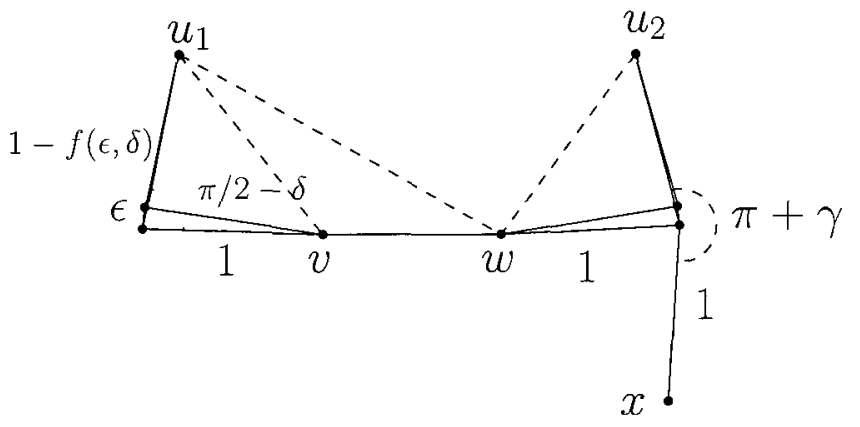

Figure 8.14: UDG Component with minimum degree 2 that requires scaling factor of $\sqrt{5}$. 


\subsubsection{Upper Bound}

Theorem 61. Let $P$ be a set of $n$ points in the plane such that $\operatorname{UDG}(P, 1)$ has minimum degree two. Then UDG $(P, \sqrt{5})$ has a 2-edge connected planar graph. Further, it can be constructed in time $O(n \log n)$.

Proof. Let $T=(P, E)$ be an MST on the set of points. Color the internal vertices of $T$ with two colors, say black and red and color leaves with green. Let $G=\left(P, E \cup E^{\prime}\right)$ be the planar graph of $T$ with minimum degree 2 obtained by Theorem 59 . Choose a chromatic class, say black. Consider a black vertex $u$ and a neighbor $v$ of $G$. It is not difficult to see that if $\{u, v\} \in E^{\prime}$, then $v$ is green, i.e., a leaf in $T$ and either $u$ was the tip of a Tie $\left(u, u^{\prime}, v, v^{\prime}\right)$ and $d(u, v) \leq \sqrt{2}$ or all the neighbors of $u$ in $T$ are in the same half-plane determined by $\{u, v\}$.

Let $u$ be a black vertex and $\{u, v\} \in E$ be a bridge of $G$. Consider the immediate edge $\{u, w\}$ of $\{u, v\}$ such that $\angle v w u<\pi$ with the preference to edges in $E$ and then edges in $E^{\prime}$.

- $\{u, w\} \in E$. Let $E^{\prime \prime}=E^{\prime \prime} \cup\{\{v, w\}\}$. Obviously $d(u, w) \leq 2$. Since $\{u, v\},\{u, w\} \in E$ the triangle $\triangle(u v w)$ is empty.

- $\{u, w\} \in E^{\prime}$. Observe that this corresponds to a Tie $\left(u, u^{\prime}, w, w^{\prime}\right)$ as depicted in Figure 8.15. We consider two cases: If $\triangle(u v w)$ is empty, then let $E^{\prime \prime}=E^{\prime \prime} \cup\{\{v, w\}\}$. Otherwise, let $p$ and $q$ be the points such that $\angle(p v u)$ and $\angle(q w u)$ are minimum. Let $E^{\prime \prime}=E^{\prime \prime} \cup\{\{v, p\},\{q, w\}\}$. Since $u$ is the tip of a Tie $\left(u, u^{\prime}, v, v^{\prime}\right)$, from Lemma 58 , $d(w, v) \leq \sqrt{5}$.

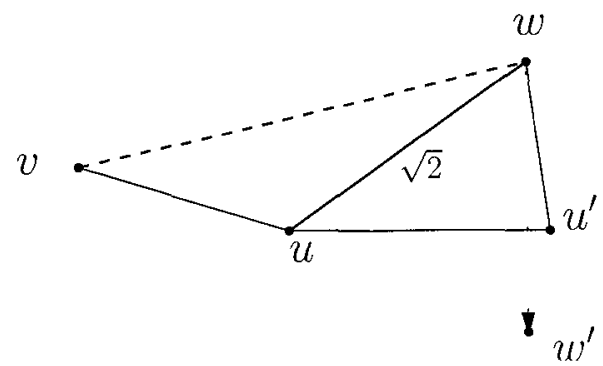

Figure 8.15: $\angle(w u v)<\pi$ and $\left\{u, v^{\prime}\right\} \in E^{\prime}$. 
It is easy to see that the new edges do not cross any edge in $E \cup E^{\prime} \cup E^{\prime \prime}$ since $\{u, w\}$ is the immediate edge of $\{u, v\}$. Further, every vertex of $G=\left(P, E \cup E^{\prime} \cup E^{\prime \prime}\right)$ is in at least one cycle. Therefore, it is two edge connected. The pseudocode is presented in Algorithm 6 . Regarding to the complexity, each new edge can be added in time $O(\log n)$. Therefore, the whole construction can be constructed in time $O(n \log n)$.

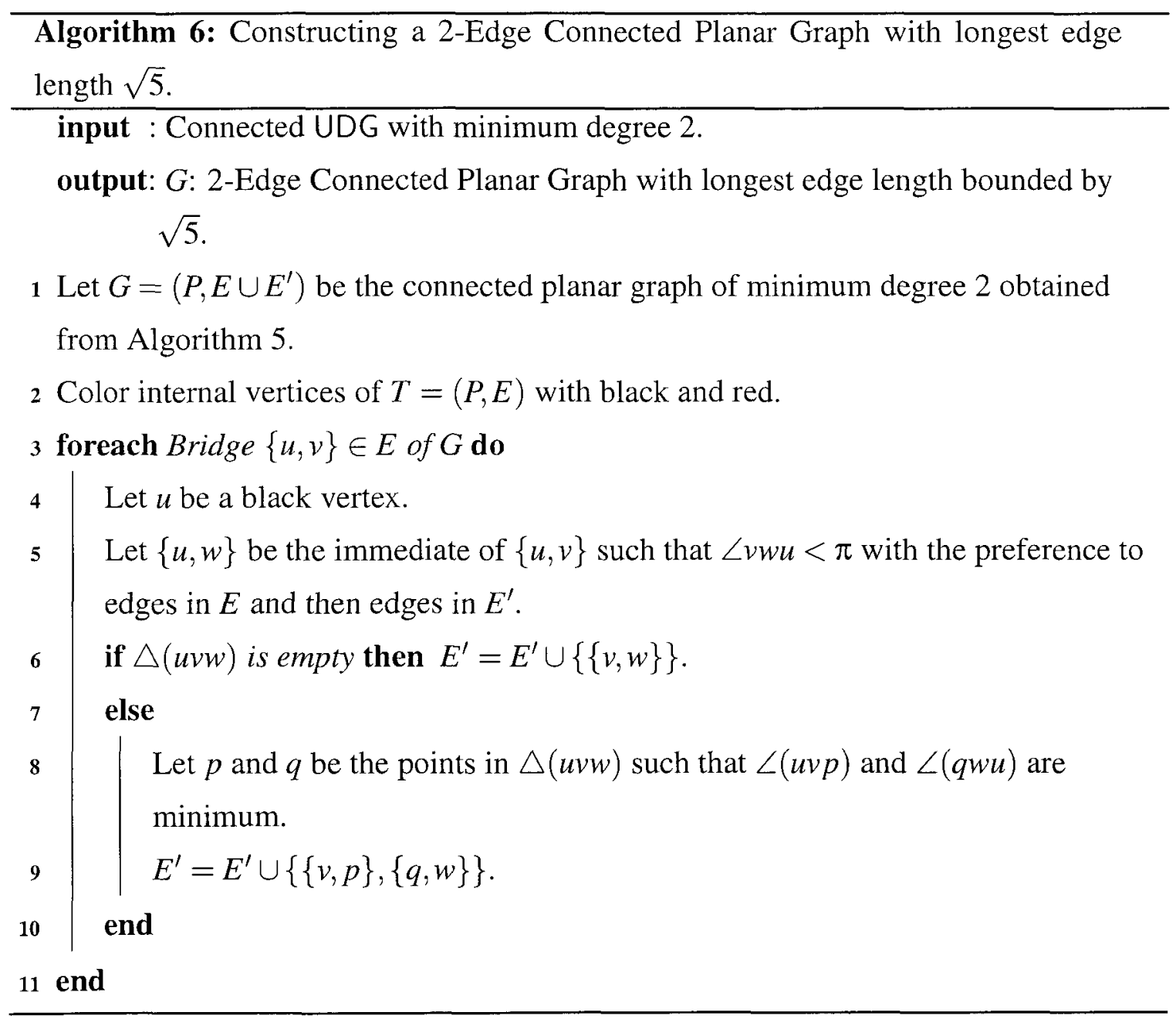

\subsection{2-Edge Connected Planar Graph of a 2-Edge Connected UDG}

In this section we will show that scaling a 2-edge connected UDG with a factor of two is always sufficient and sometimes necessary. Recall that $r_{3}$ is the minimum radius necessary 
to construct a 2-edge connected UDG. To simplify notation we assume that $r_{3}=1$. We first show the lower bound and then the upper bound.

\subsubsection{Lower Bound}

The following theorem shows that a scaling factor of 2 is sometimes necessary to create 2-edge connected planar graphs.

Theorem 62. There exists a family of 2-edge connected UDGs with $n=1+3 k$ vertices such that $\operatorname{UDG}(P, 2-\varepsilon)$ does not have a two-edge connected planar spanning subgraph.

Proof. The construction is based on the component depicted in Figure 8.16a. Observe that the component is the same as the component of Theorem 54. Clearly, it requires $\{u, v\}$ to create a 2-edge connected planar graph. A UDG with $k$ components can be created by forming a convex path as depicted in Figure 8.16b. It is not difficult to see that the lower bound also holds for this UDG with $1+3 k$ vertices.

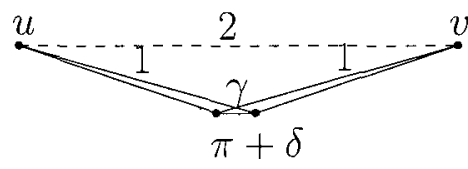

(a) Basic component that requires scaling factor of 2 .

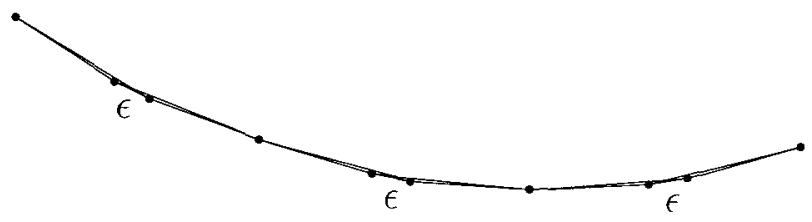

(b) Components forming a convex path with $1+3 k$ vertices.

Figure 8.16: Two-edge connected UDG with $1+3 k$ vertices that requires scaling factor of 2.

\subsubsection{Upper Bound}

We say that a vertex $v$ of a graph $G$ is arduous if $v$ has degree two, is not in a cycle and the angle that it forms with its consecutive neighbors is greater than $5 \pi / 6$. Thus, we have the following Corollary to Theorem 60 .

Corollary 63. Let $P$ be a set of $n$ points in the plane such that $\operatorname{UDG}(P, 1)$ is connected and has minimum degree 2. Further, let $T=(P, E)$ be an MST on P. Consider a two 
coloration of the vertices of $T$ with colors black and red. If $P$ does not have either black or red arduous vertices then $\operatorname{UDG}(P, 2)$ has an underlying 2-edge connected planar graph.

Proof. Let $G=\left(P, E \cup E^{\prime}\right)$ be the 2-edge connected planar graph obtained from $T$ and Theorem 61. Assume that $T$ does not have black arduous vertices. For the sake of contradiction assume that $G$ has an edge $\{v, w\} \in E^{\prime}$ such that $d(v, w)>2$. Let $u$ be the black vertex of $T$ that added $\{v, w\}$ to $G$. Observe that $u$ was the tip of a $\operatorname{Tie}\left(u ; u^{\prime}, w, w^{\prime}\right\}$ where $w$ is a leaf and the angle that $u$ forms with $u^{\prime}$ and $v$ is greater than $5 \pi / 6$. However, $T$ does not have black arduous vertices. This contradicts the assumption.

First we prove that given a 2-vertex connected $\operatorname{UDG}(P, 1)$ then $\operatorname{UDG}(P, 2)$ has an underlying 2-edge connected planar graph. Then we prove that the upper bound is also valid if the given UDG is 2-edge connected.

Theorem 64. Let $P$ be a set of $n$ points in the plane such that $\operatorname{UDG}(P, 1)$ is 2-vertex connected. Then $\operatorname{UDG}(P, 2)$ has an underlying 2-edge connected planar graph.

Proof. Let $T=(P, E)$ be the MST on $P$. Consider a two coloration of the internals vertices of $T$ with red and black colors and assign green to leaves. Choose any chromatic class, say black. If $T$ does not have black arduous vertices, then by Corollary 63, $\operatorname{UDG}(P, 2)$ has an underlying 2-edge connected planar graph. Thus, assume that $T$ has at least one black arduous vertex. We will add edges to $E^{\prime}$ in a greedy manner to obtain a graph $G=\left(P, E \cup E^{\prime}\right)$ that does not have black arduous vertices.

Consider a black arduous vertex $v$ of $G$. Let $\mathcal{G}_{1}$ and $\mathcal{G}_{2}$ be the connected components of $T \backslash v$ and $\{u, w\}$ be the edge in $\operatorname{UDG}(P, 1)$ of shortest length that connects $\mathcal{G}_{1}$ and $\mathcal{G}_{2}$. Since $\operatorname{UDG}(P, 1)$ is 2 -vertex connected, $\{u, w\}$ always exists. Assume that $u \in \mathcal{G}_{1}$ and $w \in \mathcal{G}_{2}$. Observe that every vertex in $D(u, d(u, w))$ is in $\mathcal{G}_{1}$ and every vertex in $D(w, d(u, w))$ is in $G_{2}$, otherwise $\{u, w\}$ is not the shortest edge. Therefore, $D(u, d(u, w)) \cap D(w, d(u, w))$ either is empty or contains $v$.

We will show that $\{u, w\}$ does not cross an edge of $E$. For the sake of contradiction assume that $\{u, w\}$ crosses an edge $\left\{u^{\prime}, w^{\prime}\right\} \in E$. Let $R=D(u, d(u, w)) \cap D(w, d(u, w))$. Consider first the case when $u^{\prime}$ and $w^{\prime}$ are not in $R$. Therefore, either $\angle\left(u^{\prime} u w\right)$ or $\angle\left(u w u^{\prime}\right)$ is the largest angle in $\triangle\left(u w u^{\prime}\right)$. Similarly, either $\angle\left(w u w^{\prime}\right)$ or $\angle\left(w^{\prime} w u\right)$ is the largest angle in 
$\triangle\left(u w w^{\prime}\right)$. Observe that if $\angle\left(u^{\prime} u w\right)$ and $\angle\left(w u w^{\prime}\right)$ (or $\angle\left(u w u^{\prime}\right)$ and $\angle\left(w^{\prime} w u\right)$ ) are the largest angles, then there exists a cycle $u^{\prime} w^{\prime} u$ where $d\left(u^{\prime}, w^{\prime}\right)$ is the longest edge. Therefore, $\left\{u^{\prime}, w^{\prime}\right\}$ is not in $T$. Thus, assume that $\angle\left(u^{\prime} u w\right)$ and $\angle\left(w^{\prime} w u\right)$ are the largest angles in the respective triangles as depicted in Figure 8.17a. Hence, $d\left(u^{\prime}, w^{\prime}\right)>d(u, w)$. Therefore $d\left(u^{\prime}, u\right) \leq d(u, w)$ and similarly $d\left(w^{\prime}, w\right) \leq d(u, w)$. This is a contradiction since there is a cycle $u w w^{\prime} u^{\prime} u$ where $d\left(u^{\prime}, w^{\prime}\right)$ is the largest edge length. Now consider the case when at least one vertex of $u^{\prime}$ or $w^{\prime}$ is in $R$, say $w^{\prime}$. Therefore, $v=w^{\prime}$. However, $v$ is also incident to $u$ and $w$. This is a contradiction since $d(v)=2$.

Now we will prove that if $\{u, w\}$ crosses and edge $\left\{u^{\prime}, w^{\prime}\right\} \in E^{\prime}$, then $\left\{u^{\prime}, w^{\prime}\right\}$ can be removed from $E^{\prime}$ without increasing the number of black arduous vertices in $G$. Assume without loss of generality that $u^{\prime}$ and $w^{\prime}$ are in $\mathcal{G}_{1}$ as depicted in Figure $8.17 \mathrm{~b}$, otherwise, $v$ would not be an arduous vertex. Therefore, $d(u, w) \leq \max \left(d\left(u^{\prime}, w\right), d\left(w, w^{\prime}\right)\right)$. Consider the previous step where $\left\{u^{\prime}, w^{\prime}\right\}$ was added from $G^{\prime}$. Let $v^{\prime}$ be the black arduous vertex of $G^{\prime}$ and $\mathcal{G}_{1}^{\prime}$ and $\mathcal{G}_{2}^{\prime}$ be the components of $G^{\prime} \backslash v^{\prime}$. Hence, $w$ was in either $\mathcal{G}^{\prime}{ }_{1}$ or $\mathcal{G}_{2}^{\prime}$ and either $d\left(u^{\prime}, w^{\prime}\right) \leq d\left(u^{\prime}, w\right)$ or $d\left(u^{\prime}, w^{\prime}\right) \leq d\left(w^{\prime}, w\right)$. Therefore, they form a Tie $\left(w ; u, u^{\prime}, w^{\prime}\right)$ where $u \in D\left(u^{\prime} ; d\left(u^{\prime}, w^{\prime}\right)\right) \cap D\left(w^{\prime} ; d\left(u^{\prime}, w^{\prime}\right)\right)$. Hence, $u=v^{\prime}$. Thus, if $\{u, w\}$ crosses an edge $\left\{u^{\prime}, w^{\prime}\right\} \in E^{\prime}$, then let $E^{\prime}=E^{\prime} \cup\{\{u, w\}\} \backslash\left\{\left\{u^{\prime}, w^{\prime}\right\}\right\}$. Otherwise, let $E^{\prime}=E^{\prime} \cup\{\{u, w\}\}$. Observe that any edge incident to $u$ forms an angle with either $u^{\prime}$ or $w$ or $w^{\prime}$ of at least $\pi / 3$ and any immediate neighbor $\{w, y\}$ of $\{u, w\}$ where $x, y \notin D(u ; d(u, w)) \cap D(w ; d(u, w))$ form an angle of at least $\pi / 3$.

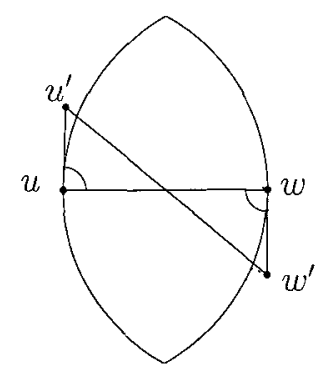

(a) $\{u, w\}$ does not cross any edge of $T$.

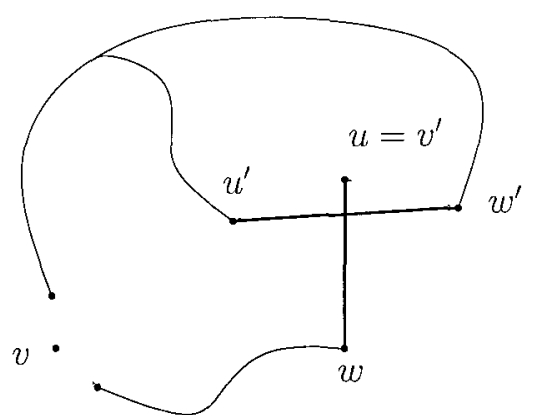

(b) If $\{u, w\} \in E^{\prime}$ crosses an edge $\left\{u^{\prime}, w^{\prime}\right\} \in E^{\prime}$, then $\left\{u^{\prime}, w^{\prime}\right\}$ can be removed.

Figure 8.17: Removing of black arduous vertices.

Clearly $G=\left(P, E \cup E^{\prime}\right)$ is planar and does not have black arduous vertices. Let $E^{\prime \prime}$ be 
the set of SNN edges of $G$.

Claim 3. Let $(u, v) \in E^{\prime \prime}$ be an edge that crosses an edge $\left\{u^{\prime}, v^{\prime}\right\} \in E^{\prime}$.

(i) If $\left\{u, u^{\prime}\right\},\left\{u, v^{\prime}\right\} \notin E$, then $\left\{u^{\prime}, v^{\prime}\right\}$ can be removed from $E^{\prime}$ without increasing black arduous vertices.

(ii) If $\left\{u, u^{\prime}\right\},\left\{v, v^{\prime}\right\} \in E$, then $\left\{u^{\prime}, v^{\prime}\right\}$ can be removed from $E^{\prime}$ without increasing black arduous vertices.

(iii) If $\left\{u, u^{\prime}\right\} \in E$ and $\left\{v, v^{\prime}\right\} \notin E$, then they form $a \operatorname{Tie}\left(v^{\prime} ; u^{\prime}, u, v\right)$.

Proof. Consider the step where $\left\{u^{\prime}, v^{\prime}\right\}$ was added from $G^{\prime}$. Let $w^{\prime}$ be the black arduous vertex of $G^{\prime}$ and let $G^{\prime}{ }_{1}$ and $G^{\prime}{ }_{2}$ be the components resulting from $G^{\prime} \backslash w^{\prime}$. Further, let $u^{\prime} \in \mathcal{G}_{1}^{\prime}$ and $v^{\prime} \in \mathcal{G}^{\prime}{ }_{2}$. Now we prove each case.

(i) Clearly $d(u, v) \leq \min \left(d\left(u, u^{\prime}\right), d\left(u, v^{\prime}\right)\right)$ since $v$ is the second nearest neighbor of $u$. Assume without loss of generality that $u \in \mathcal{G}^{\prime}{ }_{1}$. Therefore, $d\left(u^{\prime}, v^{\prime}\right)<d\left(u, v^{\prime}\right)$ and they form a $\operatorname{Tie}\left(u ; v, u^{\prime}, v^{\prime}\right)$. However, $v \in D\left(u^{\prime} ; d\left(u^{\prime}, v^{\prime}\right)\right) \cap D\left(v^{\prime} ; d\left(u^{\prime}, v^{\prime}\right)\right)$ which means that $w^{\prime}=v$. Thus, we can remove $\left\{u^{\prime}, v^{\prime}\right\}$ from $E^{\prime}$ without increasing the number of black arduous vertices in $G$; see Figure 8.18a.

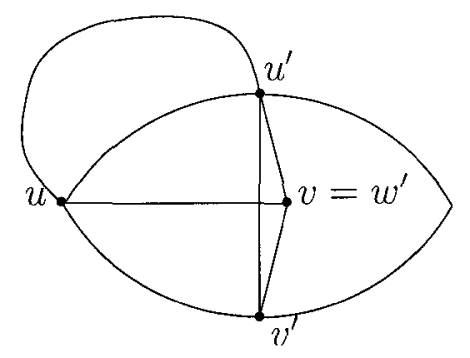

(a) If $\left\{u, u^{\prime}\right\},\left\{u, v^{\prime}\right\} \notin E$, then $\left\{u^{\prime}, v^{\prime}\right\}$ can be removed from $E^{\prime}$.

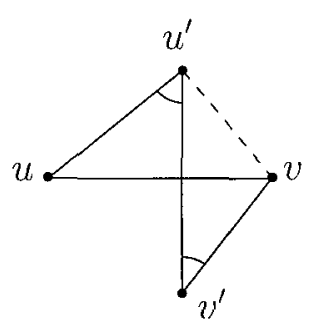

(b) If $\left\{u, u^{\prime}\right\},\left\{v, v^{\prime}\right\} \in E$, then $\left\{u^{\prime}, v^{\prime}\right\}$ can be removed from $E^{\prime}$.

Figure 8.18: $\{u, v\}$ is in at most two Ties. (Solid lines are edges of $T$ and dashed arrow lines are SNN edges.)

(ii) First we will prove that $\left\{u^{\prime}, v\right\} \in E$. By contradiction assume that $\left\{u^{\prime}, v\right\} \notin E$. Therefore, $d\left(u^{\prime}, v^{\prime}\right)<d\left(u^{\prime}, v\right)$ since $v$ is in the same component as $v^{\prime}$. Observe that $\angle\left(u u^{\prime} v^{\prime}\right)$ and $\angle\left(u^{\prime} v^{\prime} v\right)$ are the largest angles in the triangles $\triangle\left(u u^{\prime} v^{\prime}\right)$ and $\triangle\left(u^{\prime} v^{\prime} v\right)$, respectively. However, $d\left(u, v^{\prime}\right) \leq d\left(u^{\prime}, v^{\prime}\right)$ since $d\left(u^{\prime}, v\right) \geq d\left(u^{\prime}, v^{\prime}\right)$ and $\angle\left(u u^{\prime} v\right)>\angle\left(u u^{\prime} v^{\prime}\right)$. This contradicts the assumption. Hence, $\left\{u^{\prime}, v\right\} \in E$. Then $v u^{\prime} v^{\prime}$ form a cycle where $\left\{u^{\prime}, v^{\prime}\right\}$ is the 
longest edge otherwise $T$ is not minimum. Therefore, $v \in D\left(u^{\prime} ; d\left(u^{\prime}, v^{\prime}\right)\right) \cap D\left(v^{\prime} ; d\left(u^{\prime}, v^{\prime}\right)\right)$ and $w^{\prime}=v$. Thus, we can remove $\left\{u^{\prime}, v^{\prime}\right\}$ from $E^{\prime}$ without increasing the number of black arduous vertices in $G$.

(iii) First we will prove that $v \in \mathcal{G}^{\prime}{ }_{1}$. Assume by contradiction that $v$ is in $\mathcal{G}_{2}^{\prime}$. Similar to the previous case, $d\left(u^{\prime}, v^{\prime}\right)<d\left(u^{\prime}, v\right)$. Thus, $\angle\left(u u^{\prime} v^{\prime}\right)$ and $\angle\left(u^{\prime} v^{\prime} v\right)$ are the largest angles in the triangles $\triangle\left(u u^{\prime} v^{\prime}\right)$ and $\triangle\left(u^{\prime} v^{\prime} v\right)$, respectively. However, since $d\left(u^{\prime}, v\right) \geq d\left(u^{\prime}, v^{\prime}\right)$ and $\angle\left(u u^{\prime} v\right)>\angle\left(u u^{\prime} v^{\prime}\right), d\left(u, v^{\prime}\right) \leq d\left(u^{\prime}, v^{\prime}\right)$. A contradiction. Hence, $u, v \in \mathcal{G}^{\prime}{ }_{1}$ and $d\left(u^{\prime}, v^{\prime}\right) \leq$ $\min \left(d\left(v^{\prime}, u\right), d\left(v^{\prime}, v\right)\right)$. Hence, they form a Tie $\left(v^{\prime} ; u^{\prime}, u, v\right)$ since $d\left(u, v^{\prime}\right)>d(u, v)$.

From Claim 3, we can remove each edge $\left\{u^{\prime}, v^{\prime}\right\}$ of $E^{\prime}$ that crosses an edge $(u, v)$ of $E^{\prime \prime}$ such that they do not form a $\operatorname{Tie}\left(v^{\prime} ; u^{\prime}, u, v\right)$. Further, the crossings between edges in $E^{\prime \prime}$ and edges in $E \cup E^{\prime}$ are equivalent to crossings between edges in $E^{\prime \prime}$ and $E$. That is, they form Ties where leaves are endpoints of crossing lines. Thus, we can obtain a planar graph of $G=\left(P, E \cup E^{\prime} \cup E^{\prime \prime}\right)$ with minimum degree two from Theorem 59. It remains to add each bridge of $G$ into at least one cycle. Let $v$ be a black vertex of $G$ incident to a bridge $\{u, v\} \in E$ and $\{w, v\}$ be an immediate edge with the preference to edges in $E$, then in $E^{\prime}$ and then in $E^{\prime \prime}$. We have three cases:

- $\{w, v\} \in E$. Let $E^{\prime \prime}=E^{\prime \prime} \cup\{\{u, w\}\}$. Clearly, $d(u, w) \leq 2$.

- $\{w, v\} \in E^{\prime}$. We consider two cases. First assume that $w$ is red. Let $E^{\prime \prime}=E^{\prime \prime} \cup$ $\{\{u, w\}\}$. Clearly, $d(u, w) \leq 2$. Now assume that $w$ is black. Clearly $d_{G}(v) \geq 3$ and $d_{G}(w) \geq 3$. Observe that since $\{w, v\} \in E^{\prime}$ and $v$ is an internal black vertex of $T$, there exits a neighbor $w^{\prime}$ of $v$ such that $\angle\left(u v w^{\prime}\right)<\pi$ and $\left\{u, w^{\prime}\right\}$ crosses $\{v, w\}$. However, $\angle\left(w^{\prime} v w\right) \geq \pi / 3$. Therefore, $\angle(w v u) \leq 2 \pi / 3$. Let $u^{\prime}$ be the first neighbor of $w$ such that $u^{\prime} w v u$ form a convex path; see Figure 8.19. If either $u^{\prime}$ does not exist or $\left\{u^{\prime}, w\right\} \in E^{\prime}$ or $\left\{u^{\prime}, w\right\} \in E^{\prime \prime}$, then let $E^{\prime \prime}=E^{\prime \prime} \cup\{\{w, u\}\}$. Otherwise, $\left\{u^{\prime}, w\right\} \in E$. Similarly, since $\{w, v\} \in E^{\prime}$ and $w$ is an internal black vertex of $T$, there exits a neighbor $v^{\prime}$ of $w$ such that $\angle\left(u^{\prime} w v^{\prime}\right)<\pi$ and $\left\{u^{\prime}, v^{\prime}\right\}$ crosses $\{w, v\}$. However, $\angle\left(v^{\prime} w v\right) \geq \pi / 3$. Therefore, $\angle\left(u^{\prime} w v\right) \leq 2 \pi / 3$. If the quadrangle $u v w u^{\prime}$ is empty, then let $E^{\prime \prime}=E^{\prime \prime} \cup\left\{\left\{u, u^{\prime}\right\}\right\}$. Otherwise, let $p$ and $q$ be the points such that $\angle\left(p u^{\prime} w\right)$ and $\angle(q u v)$ are minimum. Let $E^{\prime \prime}=E^{\prime \prime} \cup\left\{\left\{u^{\prime}, p\right\},\{q, u\}\right\}$. It is not difficult to see 
that $d\left(u, u^{\prime}\right) \leq 2$. To see this, consider the right triangles $a u v$ and $u^{\prime} b w$ where $a$ and $b$ are the points in $\left\{u^{\prime}, u\right\}$ such that $\angle(v a u)=\pi / 2$ and $\angle\left(u^{\prime} b w\right)=\pi / 2$. From the Law of sines $d(a, u) \leq 1 / 2, d\left(u^{\prime}, b\right) \leq 1 / 2$ and $d(a, b) \leq 1$ since $\angle(a v u) \leq \pi / 6$ and $\angle\left(u^{\prime} w b\right) \leq \pi / 6$.

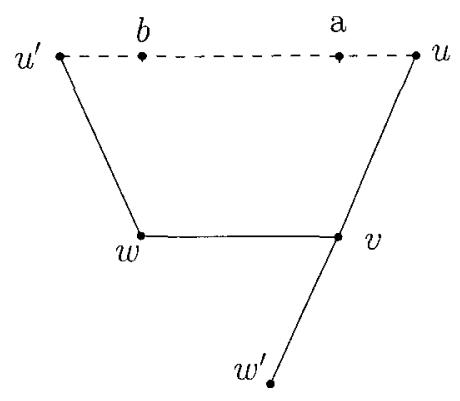

Figure 8.19: $\{w, v\} \in E^{\prime}$ and $w$ is black.

- $\{w, v\} \in E^{\prime \prime}$. We consider two cases: If $\triangle(u v w)$ is empty, then let $E^{\prime \prime}=E^{\prime \prime} \cup$ $\{\{u, w\}\}$. Otherwise, let $p$ and $q$ be the points such that $\angle(p u v)$ and $\angle(q w v)$ are minimum. Let $E^{\prime \prime}=E^{\prime \prime} \cup\{\{u, p\},\{q, w\}\}$. Since $v$ is the tip of a $\operatorname{Tie}\left(v, v^{\prime}, w, w^{\prime}\right)$ and $\angle\left(v^{\prime} v u\right) \leq 5 \pi / 6$, from Lemma $58, d(u, w) \leq 2$.

The pseudocode is presented in Algorithm 7. Regarding the time complexity, the dominating step is the removal of arduous vertices. However, it can be implemented in $O\left(n^{2}\right)$. That is, given an arduous vertex, determine the components $\mathcal{G}_{1}, \mathcal{G}_{2}$ of $G \backslash v$ in $O(n)$ time and look for the shortest edge length $\{u, w\}$ of $\operatorname{UDG}(P, 1)$ not in $G$ such that $u \in G_{1}$ and $w \in \mathcal{G}_{2}$ in $O(n)$ time. Therefore, the construction can be done in $O\left(n^{2}\right)$ time.

Theorem 65. Let $P$ be a set of $n$ points in the plane such that $\operatorname{UDG}(P, 1)$ is 2-edge connected. Then UDG $(P, 2)$ has an underlying 2-edge connected planar graph.

Proof. Consider the subsets $P_{i}$ of $P$ such that $\operatorname{UDG}\left(P_{i}, 1\right)$ is 2-vertex connected. Using Theorem 64, we can construct a 2-edge connected planar graph $G_{i}$ on $\operatorname{UDG}\left(P_{i}, 1\right)$ with length bounded by 2 since each $\operatorname{UDG}\left(P_{i}, 1\right)$ has at least three points. It is not difficult to see that $\bigcup G_{i}$ is 2-edge connected and planar. 


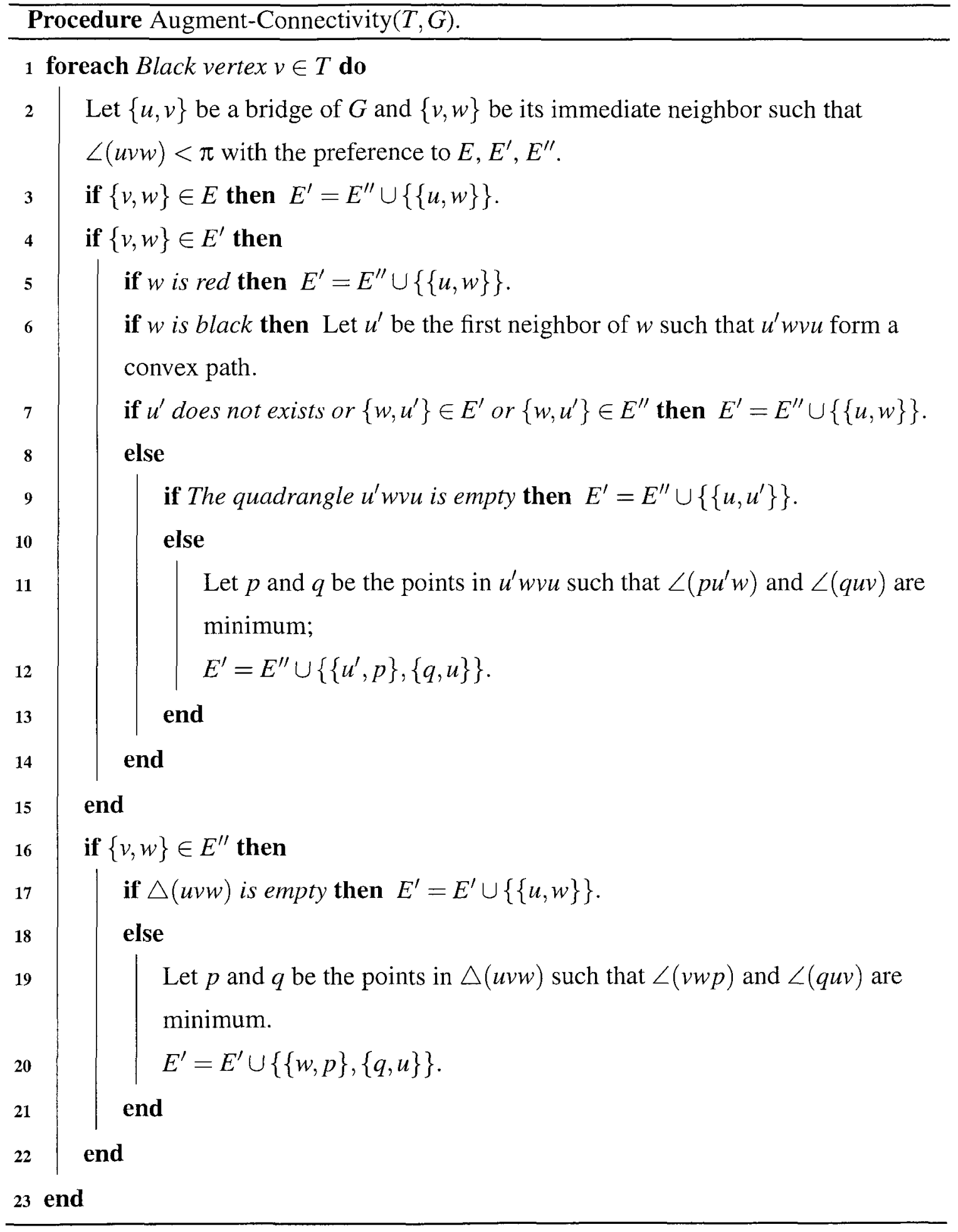

\subsection{Two-Edge Connected Planar Graphs of UDGs with high connectivity}

Intuitively one may ask whether a $k$-edge (or $k$-vertex) connected UDG has an underlying 2 -edge connected planar subgraph, for some $k>1$. We will show that this is not always 
Algorithm 7: Constructing a 2-Edge Connected Planar Graph with longest edge length bounded by 2 .

input : 2-Vertex Connected UDG.

output: $G$ : 2-Edge Connected Planar Graph with longest edge length bounded by 2 .

1 Let $T=(P, E)$ be an MST on $P, E^{\prime}=\emptyset$ and $G=\left(P, E \cup E^{\prime}\right)$.

2 Color the internal vertices of $T$ with black and red colors.

3 Let $A$ be the set of black arduous vertices of $T$.

4 while $A$ is not empty do

Let $v$ be a vertex of $A$ and $\mathcal{G}_{1}, \mathcal{G}_{2}$ be the components of $G \backslash v$.

6 Let $\{u, w\}$ be the shortest edge such that $u \in \mathcal{G}_{1}$ and $w \in \mathcal{G}_{2}$ if $\{u, w\}$ crosses an edge $\left\{u^{\prime}, w^{\prime}\right\} \in E^{\prime}$ then Let $E^{\prime}=E^{\prime} \cup\{\{u, w\}\} \backslash\left\{\left\{u^{\prime}, w^{\prime}\right\}\right\}$. else Let $E^{\prime}=E^{\prime} \cup\{\{u, w\}\}$.

9 Remove the vertices of $A$ that are not longer arduous in $G$.

10 end

11 Let $E^{\prime \prime}$ be the SNN edges of $G$ and remove each edge $\left\{u^{\prime}, v^{\prime}\right\}$ of $E^{\prime}$ that crosses an edge $(u, v)$ of $E^{\prime \prime}$ such that they do not form a Tie $\left(v^{\prime} ; u^{\prime}, u, v\right)$.

12 Let $G=\left(P, E \cup E^{\prime} \cup E^{\prime \prime}\right)$ be the connected planar graph of minimum degree 2 obtained from Algorithm 5.

13 Call Augment-Connectivity $(T, G)$;

true.

Theorem 66. There exist $k$-vertex connected UDGs that do not have 2-edge connected planar subgraphs, for any $k=O(\sqrt{n})$ even for a scaling factor of $17 / 16$.

Proof. Assume $k=2 m$. Consider the $C^{k}$ and the wire components depicted in Figure 8.20a and Figure $8.20 \mathrm{~b}$ with $2 k+2$ vertices and $2 k$ vertices respectively. It is easy to see that $C^{k}$ is a valid two-vertex connected UDG and the wire is a valid $k$-vertex connected UDG. Observe that $C^{k}$ does not have a 2-edge connected planar subgraph since the inclusion of $\left\{u_{1}, u_{k}^{\prime}\right\}$ or $\left\{u_{1}^{\prime}, u_{k}\right\}$ leaves $v^{\prime}$ or $v$ with degree one respectively. Hence, we call $v$ and $v^{\prime}$ the isolated vertices of $C^{k}$. Observe that we can embed $C^{k}$ in such a way that the distances $d\left(v, u_{k}\right), d\left(u_{k}, u_{k-1}\right), d\left(u_{2}, u_{1}\right)$ and $d\left(v^{\prime}, u_{k}^{\prime}\right), d\left(u_{k}^{\prime}, u_{k-1}^{\prime}\right), d\left(u_{2}^{\prime}, u_{1}^{\prime}\right)$ are at least $\frac{1}{4}-$ $\varepsilon$. Hence, $d\left(u_{1}^{\prime}, v\right)=d\left(u_{1}^{\prime}, u_{2}\right)=d\left(u_{1}, v^{\prime}\right)=d\left(u_{1}, u_{2}^{\prime}\right) \sim 17 / 16$. Let $C_{l}^{k}$ be $m$ consecutive 
$C^{k}$ components in such a way that they are at distance greater than one from each other. We can connect the upper and lower part of $C_{i}^{k}$ with $C_{i+1}^{k}$ with a constant number of wires, i.e., $k$ independent paths that connect the upper and the lower part of $C_{i}^{k}$ and $C_{i+1}^{k}$ in such a way that the isolated vertices of each $C_{i}^{k}$ are far from the wires as depicted in Figure 8.20a. It is easy to see that the resulting graph is $k$-vertex connected, has $O\left(k^{2}\right)$ vertices and does not have an underlying two-edge planar graph. The theorem follows.

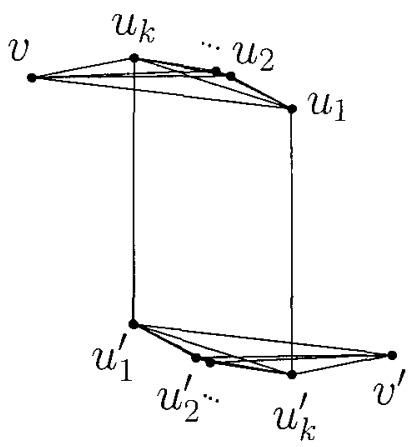

(a) $C^{k}$ component.

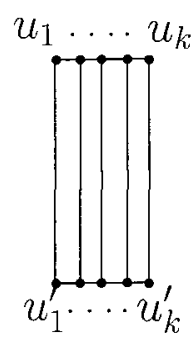

(b) Wire.

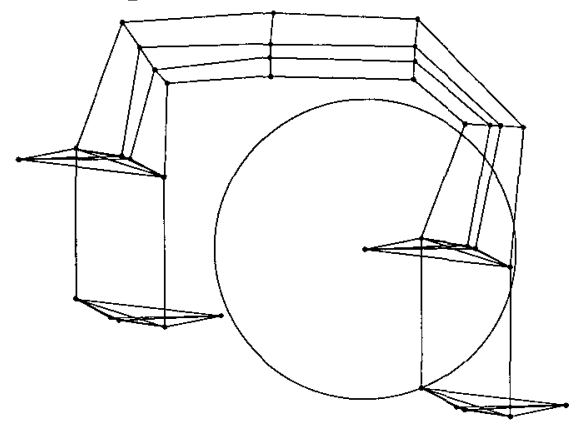

(c) Upper connection between $C_{t}^{k}$ and $C_{l+1}^{k}$.

Figure 8.20: $k$-vertex connected UDG that does not have 2-edge connected planar subgraph.

\subsection{Conclusion}

In this chapter, we have shown that for any given point set in the plane, there exits a two edge connected planar graph that spans the points with edge length bounded by 2 times the optimal radius. 


\section{Chapter 9}

\section{Conclusion and Further Research}

The motivation of this thesis was to provide answers to the problem of replacing omnidirectional antennae with directional antennae. We gave positive results in the form of upper bounds and negative results in the form of hardness statements to the main question as well as related problems. In what follows, we look back at the results and provide direction for further work.

In Chapter 3, we have explored the antenna orientation problem in $2 \mathrm{D}$. We provided an algorithm to orient up to $k$ directional antennae to create a strongly connected graph from a set of sensors in the plane in such a way that the transmission range is bounded by $2 \sin \left(\frac{\pi}{k+1}\right)$ times the optimal transmission range. Such an algorithm is valid for any given angle of the directional antennae. We also gave an NP-Completeness result when each sensor has two antennae.

Let $r_{k}(\varphi)$ be the optimal radius when $k$ directional antennae are used per sensor such that the sum of angles is at most $\varphi$. Let $r(T)$ be the longest edge length of the MST on a set of points. Table 9.1 shows our conjectured values for the antenna orientation problem.

\begin{tabular}{c|cc|cc|cc}
\hline \multirow{2}{*}{ \# of Antennae } & \multicolumn{2}{|c}{ Lower Bound } & \multicolumn{2}{c}{ Upper Bound } & \multicolumn{2}{c}{ Conjecture } \\
\cline { 2 - 7 } & Angle & Radius & Angle & Radius & Angle & Radius \\
\hline 1 & $2 \pi / 3$ & $\sqrt{3} \cdot r_{1}(2 \pi / 3)$ & $\pi$ & $\sqrt{3} \cdot r(T)$ & $2 \pi / 3$ & $2 \cdot r(T)$ \\
2 & $9 \pi / 20$ & $1.3 \cdot r_{2}(9 \pi / 20)$ & $2 \pi / 3$ & $\sqrt{2} \cdot r(T)$ & $\pi / 2$ & $\sqrt{2} \cdot r(T)$ \\
3 & - & - & 0 & $\sqrt{2} \cdot r(T)$ & 0 & $r_{3}(0)$ \\
4 & - & - & 0 & $2 \sin (\pi / 5) \cdot r(T)$ & 0 & $r_{4}(0)$ \\
\hline
\end{tabular}

Table 9.1: Conjectured values for the antenna orientation problem.

We can consider a variety of different problems for the antenna orientation problem by imposing distinct constraints on the resulting graph. An interesting problem is to assume that sensors have also directional receivers. This allows us to increase the transmission range even further. A particular case is when directional receivers have angle zero in 
which case the in-degree is bounded. A different problem is to impose stretch factor on the resulting graph. This problem was considered before in [16] for one antenna per sensor. However, it is open for multiple directional antennae. Moreover, no non-trivial lower bounds are known.

It is also of interest to study this problem in distinct settings when the angle of the directional antennae is zero. For example, if the transmission range is not homogeneous or for distinct metric spaces. A doubling metric space might be a good starting point since it generalizes different settings including heterogeneous transmission ranges [62].

In Chapter 4 we have explored the antennae orientation problem in 3D. We consider the case when each sensor has one antenna. We provided a deterministic algorithm to create a strongly connected network with optimal range when the solid angle $\Omega$ is at least $18 \pi / 5$ and an approximation algorithm when the solid angle $\Omega$ is between $2 \pi$ and $18 \pi / 5$. We also gave an NP-Completeness result when the solid angle is less than $\pi$. In addition to improving the results presented in this chapter, several interesting questions remain open. For once, nothing is known when the spherical antenna angle $\Omega$ is between $\pi$ and $2 \pi$. Another question is concerned with angle/range trade-offs when each sensor is equipped with a given number $k$ of antennae, where $1 \leq k$. The problem becomes trivial when $k \geq 12$ since the maximum degree of any MST in 3D is 12.

In Chapter 5, we explored the graph orientation problem. We focused on 2-edge connected planar graphs. We provided algorithms to orient directional antennae of a given geometric graph in such a way that the shortest path between any two points is bounded by a multiplicative factor of the shortest path in the original graph. We proved that the multiplicative factor depends only on the size of the faces. However, the lower bound remains as an open problem. It is also of interest to look at trade-offs when directional antennae with angle greater than zero are used as well as the weighted version of the problem.

The results of the graph orientation problem raise the problem of constructing 2-edge connected graphs with bounded length. In Chapter 6 we have explored this problem using the augmentation approach. We provided a set of algorithms to augment the connectivity of a geometric planar graph into two-edge connected planar graphs with edges of length bounded by three times the longest edge of the original graph. An open problem is a 
full characterization of the instances of geometric planar graphs that allow being augmented into $k_{v}$-vertex connected planar graphs and/or $k_{e}$-edge connected planar graphs with bounded length; where $2 \leq k_{v} \leq 5$ and $3 \leq k_{e} \leq 5$.

A different approach is to obtain a 2-edge connected planar subgraph of a given UDG. To decide whether a UDG has such a graph, first we need to decide whether the UDG has a planar graph of minimum degree two. We studied this problem in Chapter 7. We proved that this problem is NP-Complete as well if we ask for a planar graph of minimum degree 3. This problem is also hard for planar subgraphs of qUDGs with minimum degree one. However, it remains open whether a UDG has a planar subgraph of minimum degree 4 as well as minimum degree 5. Further, what are the effects in the scaling factor when we also consider connectivity.

In Chapter 8, we provided algorithms to construct 2-edge connected planar graphs with longest edge length that differs by a constant multiplicative factor of the optimal radius. It is unknown how to construct $k_{v}$-vertex (or $k_{e}$-edge) connected planar graph with optimal (or approximation) length on a set of points for $2 \leq k_{v} \leq 5$ and $3 \leq k_{e} \leq 5$. 


\section{Nomenclature}

A-Tree Antenna Tree

DT Delaunay Triangulation

MST Minimum Spanning Tree

qUDG quasi Unit Disk Graph

ROMA Receiver-Oriented Multiple Access

SNN Second Nearest Neighbor

UBG Unit Ball Graph

UDG Unit Disk Graph

WSN Wireless Sensor Networks 


\section{Index}

Antenna

Directional, 1

Omnidirectional, 1

Range, 1

Spread, 1

Antenna-Tree, 21

Arduous Vertex, 118

Bow, 105

Bridge, 6

Consecutive neighbors, 21

Cut-vertex, 6

Geometric Graph, 5

$\alpha$ th power, 21

$k$-edge connected, 6

$k$-vertex connected, 6

Face coloration, 57

Orientation of a graph, 6

Planar, 6

quasi Unit Disk Graph, 6

Stretch factor, 6

Unit Ball Graph, 47

Unit Disk Graph, 5

Kissing Number, 7

Minimum Spanning Tree, 6

Euclidean, 6

Neighbor

Immediate neighbor, 70
Second Nearest Neighbor, 106

Orientation of planar circuit network, 87

Planar 3SAT, 89

Planar circuit network, 86

Problem

Antenna Orientation, 7

Augmentation with Bounded Length, 8

Graph Orientation, 8

Min Degree Planar Subgraph, 8

Optimal Length $k$-Connected Planar Graph, 9

Sensors, 1

Tammes' radius, 48

Tie, 103

Wireless Sensor Networks, 1 


\section{Bibliography}

[1] M. Abellanas, A. García, F. Hurtado, J. Tejel, and J. Urrutia. Augmenting the connectivity of geometric graphs. Computational Geometry: Theory and Applications, 40(3):220-230, 2008.

[2] P. Agarwal, H. Edelsbrunner, O. Schwarzkopf, and E. Welzl. Euclidean minimum spanning trees and bichromatic closest pairs. Discrete and Computational Geometry, 6(5):407-422, 1991.

[3] M. Al-Jubeh, M. Ishaque, K. Rédei, D. Souvaine, and C. Tóth. Tri-edge-connectivity augmentation for planar straight line graphs. In 20th International Symposium on Algorithms and Computation (ISAAC 2009). LNCS 5878, pages 902-912, Hawaii, USA, Dec 16-18 2009. Springer.

[4] L. Bao and J.J. Garcia-Luna-Aceves. Transmission scheduling in ad hoc networks with directional antennas. In 8th annual international conference on Mobile computing and networking (MobiCom '02), pages 48-58. ACM New York, NY, USA, 2002.

[5] L. Barrière, P. Fraigniaud, L. Narayanan, and J. Opatrny. Robust position-based routing in wireless ad hoc networks with unstable transmission ranges. Wireless Communications and Mobile Computing Journal, 3(2):141-153, 2003.

[6] M. de Berg, M. van Kreveld, M. Overmars, and Schwarzkopf O. Computational Geometry: Algorithms and Applications. Springer-Verlag, second edition edition, 2000.

[7] B. Bhattacharya, Y Hu, E. Kranakis, D. Krizanc, and Q. Shi. Sensor network connectivity with multiple directional antennae of a given angular sum. In 23rd IEEE International Parallel and Distributed Processing Symposium (IPDPS 2009), Rome, Italy, May 25-29 2009. IEEE Press.

[8] V. Borodin. On acyclic colorings of planar graphs. Discrete Mathematics, 25(3):211236, 1979.

[9] T. Calamoneri and R. Petreschi. An efficient orthogonal grid drawing algorithm for cubic graphs. In First Annual International Conference on Computing and Combinatorics (COCOON '95), pages 31-40. Springer-Verlag, 1995.

[10] I. Caragiannis, C. Kaklamanis, E. Kranakis, D. Krizanc, and A. Wiese. Communication in wireless networks with directional antennae. In 20th ACM Symposium on Parallelism in Algorithms and Architectures (SPAA'08), pages 344-351, Munich, Germany, June 14-16 2008. IEEE/ACM. 
[11] J. Cheriyan, A. Sebö, and Z. Szigeti. An improved approximation algorithm for minimum size 2-edge connected spanning subgraphs. In 6th International Conference on Integer Programming and Combinatorial Optimization (IPCO), volume 1412, pages 126-136. Springer Berlin / Heidelberg, 1998.

[12] E.J. Cockayne and S.T. Hedetniemi. Optimal domination in graphs. IEEE Transactions on Circuits and Systems, 22(11):855-857, 1975.

[13] H.T. Croft, K.J. Falconer, and R.K. Guy. Unsolved problems in geometry. Springer, 1991.

[14] J. Czyzowicz, S. Dobrev, T. Fevens, H. González-Aguilar, E. Kranakis, J. Opatrny, and J. Urrutia. Local algorithms for dominating and connected dominating sets of unit disk graphs. In 8th Latin American conference on Theoretical informatics (LATIN 2008). LNCS 4957, pages 158-169, Búzios, Rio de Janeiro, Brazil, April 7-11 2008. Springer, LNCS.

[15] J. Czyzowicz, S. Dobrev, H. Gonzalez-Aguilar, R. Kralovic, E. Kranakis, J. Opatrny, L. Stacho, and J. Urrutia. Local 7-coloring for planar subgraphs of unit disk graphs. In 5th international conference on Theory and applications of models of computation (TAMC 2008), LNCS 4978, pages 170-181, Xi'an, China, April 25-29 2008. Springer-Verlag.

[16] M. Damian and R. Flatland. Spanning properties of communication graphs induced by directional antennas. In Electronic Proceedings of the 20th Fall Workshop on Computational Geometry, Stony Brook University, Stony Brook, NY, October 29 2010.

[17] S. Dobrev, E. Kranakis, D. Krizanc, O. Morales Ponce, J. Opatrny, and L. Stacho. Strong connectivity in sensor networks with given number of directional antennae of bounded angle. In Proceedings of the 4th Annual International Conference on Combinatorial Optimization and Applications (COCOA 10). Part II, LNCS 6509, pages 72-86, Big Island, Hawaii, Dec 18-20 2010. Springer-Verlag.

[18] S. Dobrev, E. Kranakis, D. Krizanc, O. Morales Ponce, and L. Stacho. Approximating the edge length of 2-edge connected planar geometric graphs in udgs, 2011.

[19] Q. Dong and Y. Bejerano. Building robust nomadic wireless mesh networks using directional antennas. In 27th Conference on Computer Communications (INFOCOM 2008), pages 1624-1632, Phoenix, AZ, USA, April 12-18 2008.

[20] D. Eppstein. Spanning trees and spanners. In Jörg-Rudiger Sack and Jorge Urrutia, editors, Handbook of Computational Geometry, chapter 9, pages 425-461. Elsevier, 2000 .

[21] K.P. Eswaran and R.E. Tarjan. Augmentation problems. SIAM Journal on Computing, 5(4):653-665, 1976. 
[22] A. Francke and M. Hoffmann. The euclidean degree-4 minimum spanning tree problem is np-hard. In 25th annual Symposium on Computational Geometry (SCG '09), pages 179-188, Aarhus University, Denmark, June 8-10 2009. ACM New York, NY, USA.

[23] T. Fukunaga. Graph orientations with set connectivity requirements. In 20th International Symposium on Algorithms and Computation (ISAAC '09). LNCS 5878, pages 265-274, Hawaii, USA, 2009. Springer-Verlag.

[24] K.R. Gabriel and R.R. Sokal. A new statistical approach to geographic variation analysis. Systematic Zoology, 18(3):259-278, 1969.

[25] A. García, F. Hurtado, C. Huemer, J. Tejel, and P. Valtr. On triconnected and cubic plane graphs on given point sets. Computational Geometry, 42(9):913-922, 2009.

[26] M.R. Garey and D.S. Johnson. Computers and Intractability. A Guide to the Theory of NP-Completeness. Series of Books in the Mathematical Sciences. WH Freeman and Company, San Francisco, California, 1979.

[27] P. Gupta and P.R. Kumar. The capacity of wireless networks. Information Theory, IEEE Transactions on, 46(2):388-404, 2000.

[28] L. Hu and D. Evans. Using directional antennas to prevent wormhole attacks. In Network and Distributed System Security Symposium (NDSS'04), pages 131-141. Internet Society, 2004.

[29] H. Imai, K. Kobara, and K. Morozov. On the possibility of key agreement using variable directional antenna. In Ist Joint Workshop on Information Security (JWIS 2006), Sookmyung Womens University, Seoul Korea, Sep 20-21 2006. IEICE.

[30] B. Jackson and T. Jordán. Independence free graphs and vertex connectivity augmentation. Journal Combinatory Theory, Series B, 94(1):31-77, 2005.

[31] T.R. Jensen and B. Toft. Graph coloring problems. Wiley-Interscience, New York, 1996.

[32] R. Jothi, B. Raghavachari, and S. Varadarajan. A 5/4-approximation algorithm for minimum 2-edge-connectivity. In Fourteenth Annual ACM-SIAM Symposium on Discrete Algorithms (SODA '03), pages 725-734, Baltimore, MD, USA, Jan 12-14 2003. Society for Industrial and Applied Mathematics.

[33] G. Kant. Augmenting outerplanar graphs. Journal of Algorithms, 21(1):1-25, 1996.

[34] G. Kant and H. Bodlaender. Planar graph augmentation problems. In Workshop on Algorithms and Data Structures (WADS '91). LNCS 519, pages 286-298, Ottawa, Canada, Aug 14-16 1991. Springer. 
[35] S. Khuller, B. Raghavachari, and N. Young. Balancing minimum spanning trees and shortest-path trees. Algorithmica, 14(4):305-321, 1995.

[36] E. Kranakis, D. Krizanc, A. Modi, and O. Morales Ponce. Maintaining connectivity in 3D wireless sensor networks using directional antennae. In 25th IEEE International Parallel \& Distributed Processing Symposium (IPDPS 2011), Anchorage, Alaska, USA, May 16-20 2011. IEEE Press.

[37] E. Kranakis, D. Krizanc, and O. Morales. Maintaining connectivity in sensor networks using directional antennae. In S. Nikoletseas and J. Rolim, editors, Theoretical aspects of Distributed Computing in Sensor Networks, chapter 3, pages 59-84. Springer, 2010. ISBN 978-3-642-14848-4.

[38] E. Kranakis, D. Krizanc, O. Morales Ponce, and L. Stacho. Bounded length, 2-edge augmentation of geometric planar graphs. In Proceedings of the 4th Annual International Conference on Combinatorial Optimization and Applications (COCOA 10). Part I, LNCS 6509, pages 385-397, Big Island, Hawaii, Dec 18-20 2010. SpringerVerlag.

[39] E. Kranakis, D. Krizanc, and J. Urrutia. Coverage and connectivity in networks with directional sensors. In Euro-Par Conference. LNCS 3149, pages 917-924, Pisa, Italy, Aug 31-Sep 3 2004. Springer.

[40] E. Kranakis, D. Krizanc, and E. Williams. Directional versus omnidirectional antennas for energy consumption and k-connectivity of networks of sensors. In 8th International Conference on Principles of Distributed Systems (OPODIS 2004). LNCS 3544, pages 357-368, Grenoble, France, Dec 15-17 2005. Springer.

[41] E. Kranakis, O. Morales Ponce, and L. Stacho. Strong orientations of planar graphs with bounded stretch factor. In 17th Structural Information and Communication Complexity (SIROCCO 2010). LNCS 6058, pages 224-236, Sirince, Turkey, Jun $7-$ 11 2010. Springer-Verlag.

[42] E. Kranakis, O. Morales Ponce, and J. Suomela. Planar subgraphs without lowdegree nodes. In Algorithms And Data Structures Symposium (WADS 2011), Polytechnic Institute of New York University, Brooklyn, NY, USA, Aug 15172011.

[43] E. Kranakis, H. Singh, and J. Urrutia. Compass routing on geometric networks. In 11 th Canadian Conference on Computational Geometry, Vancouver, British Columbia, Canada, Aug 15-18 1999.

[44] J.B. Krukal Jr. On the shortest spanning subtree of a graph and the travelling salesman problem. In American Mathematical society, volume 7(1), pages 48-50. American Mathematical Society, 1956. 
[45] M. Kuorilehto, M. Hännikäinen, and T.D. Hämäläinen. A survey of application distribution in wireless sensor networks. EURASIP Journal on Wireless Communications and Networking, 5(5):774-788, 2005.

[46] S. Kutten and D. Peleg. Fast distributed construction of small $k$-dominating sets and applications. Journal of Algorithms, 28(1):40-66, 1998.

[47] X. Li, Y. Wang, and W. Song. Applications of k-local mst for topology control and broadcasting in wireless ad hoc networks. IEEE Transactions on Parallel and Distributed Systems, 15(12):1057-1069, 2004.

[48] D. Lichtenstein. Planar formulae and their uses. SIAM Journal on Computing, 11(2):329-343, 1982.

[49] A. Liebers. Planarizing graphs - a survey and annotated bibliography. Journal of Graph Algorithms and Applications, 5(1):1-74, 2001.

[50] X. Lu, F. Wicker, P. Lio, and D. Towsley. Security estimation model with directional antennas. In IEEE Military Communications Conference (MILCOM 2008), pages 1-6, San Diego, CA, USA, Nov 17-19 2008.

[51] C. Monma and S. Suri. Transitions in geometric minimum spanning trees. Discrete and Computational Geometry, 8(1):265-293, 1992.

[52] C.S.J.A. Nash-Williams. On orientations, connectivity and odd vertex pairings in finite graphs. Canadian Journal of Mathematics, 12:555-567, 1960.

[53] V. Navda, A.P. Subramanian, K. Dhanasekaran, A. Timm-Giel, and S. Das. Mobisteer: using steerable beam directional antenna for vehicular network access. In 5th international conference on Mobile systems, applications and services (MobiSys '07), pages 192-205, San Juan, Puerto Rico, June 11-14 2007. ACM.

[54] R.G. Parker and R.L. Rardin. Guaranteed performance heuristics for the bottleneck traveling salesman problem. Operations Research Letters, 2(6):269-272, 1984.

[55] D. Peleg. Distributed computing: a locality-sensitive approach. Society for Industrial and Applied Mathematics Philadelphia, PA, USA, 2000.

[56] F. Pfender and G.M. Ziegler. Kissing numbers, sphere packings, and some unexpected proofs. Notices-American Mathematical Society, 51:873-883, 2004.

[57] R.C. Prim. Shortest connection networks and some generalizations. Bell system technical journal, 36(6):1389-1401, 1957.

[58] R. Ramanathan. On the performance of ad hoc networks with beamforming antennas. In 2nd ACM international symposium on Mobile ad hoc networking \& computing (MobiHoc '01), pages 95-105, Long Beach, CA, USA, Oct 04-05 2001. ACM Press New York, NY, USA. 
[59] N. Robertson, D. Sanders, P. Seymour, and R. Thomas. The four-colour theorem. Journal of Combinatorial Theory, Series B, 70(1):2-44, 1997.

[60] G. Robins and J.S. Salowe. Low-degree minimum spanning trees. Discrete and Computational Geometry, 14(1):151-165, 1995.

[61] I. Rutter and A. Wolff. Augmenting the connectivity of planar and geometric graphs. Electronic Notes in Discrete Mathematics, 31:53-56, 2008.

[62] S. Schmid and R. Wattenhofer. Algorithmic models for sensor networks. In 20th IEEE International Parallel and Distributed Processing Symposium (IPDPS 2006), page 11, Rhodes Island, Greece, Apr 25-29 2006. IEEE.

[63] M. Shamos. Computational Geometry. PhD thesis, Dept. Computer Science, Yale University, 1978.

[64] A. Spyropoulos and C. S. Raghavendra. Capacity bounds for ad-hoc networks using directional antennas. In IEEE International Conference on Communications (ICC'03), pages 348-352, Anchorage, Alaska, USA, May 11-15 2003. IEEE.

[65] A. Spyropoulos and C.S. Raghavendra. Energy efficient communications in ad hoc networks using directional antennas. In Twenty-First Annual Joint Conference of the IEEE Computer and Communications Societies (INFOCOM 2002), pages 220-228, New York, USA, June 23-27 2002. IEEE.

[66] P.M.L. Tammes. On the origin of number and arrangement of the places of exit on the surface of pollen grains. Recueil Traveaux Botaniques Néerlandais, 27:1-84, 1930.

[67] T. Tarnai and Z. Gáspár. Covering a sphere by equal circles, and the rigidity of its graph. Mathematical Proceedings of the Cambridge Philosophical Society, 110(1):71-89, 1991.

[68] C. Tóth. Connectivity augmentation in plane straight line graphs. Electronic Notes in Discrete Mathematics, 31:49-52, 2008.

[69] C.D. Tóth and P. Valtr. Augmenting the edge connectivity of planar straight line graphs to three. In 13th Spanish Meeting on Computational Geometry, Zaragoza, 2009.

[70] T. Toussaint. The relative neighbourhood graph of a finite planar set. Pattern Recognition, 12(4):261-268, 1980.

[71] S. Vempala and A. Vetta. Factor 4/3 approximations for minimum 2-connected subgraphs. In Third International Workshop on Approximation Algorithms for Combinatorial Optimization (APPROX 'O0), pages 262-273, London, UK, 2000. SpringerVerlag. 
[72] T. Watanabe and A. Nakamura. Edge-connectivity augmentation problems. Journal of Computer and System Sciences, 35(1):96-144, 1987.

[73] A. Wiese and E. Kranakis. Local ptas for independent set and vertex cover in location aware unit disk graphs. In IEEE International Conference on Distributed Computing in Sensor Systems (DCOSS 2008). LNCS 5067, pages 415-431, Santorini Island, Greece, June 11-14 2008. Springer-Verlag.

[74] S. Yi, Y. Pei, and S. Kalyanaraman. On the capacity improvement of ad hoc wireless networks using directional antennas. In 4th ACM international symposium on Mobile ad hoc networking \& computing (MobiHoc '03), pages 108-116, Annapolis, MD, USA, June 1-3 2003. ACM Press New York, NY, USA.

[75] J. Yick, B. Mukherjee, and D. Ghosal. Wireless sensor network survey. Computer Networks, 52(12):2292-2330, 2008.

[76] H. Zhang and X. He. On even triangulations of 2-connected embedded graphs. SIAM Journal on Computing, 34(3):683-696, 2005. 